\title{
Chronic pain and pain perception
}

Citation for published version (APA):

Peters, M. L. (1992). Chronic pain and pain perception. [Doctoral Thesis, Maastricht University]. Datawyse / Universitaire Pers Maastricht. https://doi.org/10.26481/dis.19920522mp

Document status and date:

Published: 01/01/1992

DOI:

10.26481/dis.19920522mp

Document Version:

Publisher's PDF, also known as Version of record

\section{Please check the document version of this publication:}

- A submitted manuscript is the version of the article upon submission and before peer-review. There can be important differences between the submitted version and the official published version of record.

People interested in the research are advised to contact the author for the final version of the publication, or visit the DOI to the publisher's website.

- The final author version and the galley proof are versions of the publication after peer review.

- The final published version features the final layout of the paper including the volume, issue and page numbers.

Link to publication

\footnotetext{
General rights rights.

- You may freely distribute the URL identifying the publication in the public portal. please follow below link for the End User Agreement:

www.umlib.nl/taverne-license

Take down policy

If you believe that this document breaches copyright please contact us at:

repository@maastrichtuniversity.nl

providing details and we will investigate your claim.
}

Copyright and moral rights for the publications made accessible in the public portal are retained by the authors and/or other copyright owners and it is a condition of accessing publications that users recognise and abide by the legal requirements associated with these

- Users may download and print one copy of any publication from the public portal for the purpose of private study or research.

- You may not further distribute the material or use it for any profit-making activity or commercial gain

If the publication is distributed under the terms of Article $25 \mathrm{fa}$ of the Dutch Copyright Act, indicated by the "Taverne" license above, 


\section{CHRONIC PAIN \\ AND \\ PAIN PERCEPTION}





\section{CHRONIC PAIN \\ AND \\ PAIN PERCEPTION}

\section{PROEFSCHRIFT}

ter verkrijging van de graad van doctor aan de Rijksuniversiteit Limburg te Maastricht, op gezag van de Rector Magnificus, Prof. mr. M.J. Cohen, volgens het besluit van het College van Dekanen, in het openbaar te verdedigen

op vrijdag, 22 mei 1992 om 16.00 uur

door

Madelon Louise Peters 
Promotor:

Co-promotor:

Beoordelingscommissie:
Prof. Dr, M.A. wan den Hout

Dr. A.J.M. Schmidt

Prof. Dr. J. Jolles (voorzitter)

Prof. Dr. W.T.A.M. Everaerd (Universiteit van Amsterdam)

Prof. Dr. D. Le Bars (INSERM, Paris)

Prof. Dr. R.S. Reneman

Prof. Dr. M.E. Sluiliter

CIP-GEGEVENS KONINKLIJKE BIBLIOTHEEK, DEN HAAG

Peters, Madelon Louise

Chronic pain and pain perception / Madelon Louise Peters.

- Maastricht: Universitaire Pers Maastricht - III.

Proefschrift Maastricht. - Met lit. opg. - Met

samenvatting in Nederlands.

ISBN $90-5278-023-4$

NUGI 745

Trefw. : pijin ; psychofysiology.

Het verschijnen van dit proefschrift werd mede mogelijk gemaakt door financiële steun van:

- Schering-Plough BV, Postbus 70, $1180 \mathrm{AB}$ Amstelveen

- Samofi Winthrop v.o.f., Postbus 97, 3140 AB Maassluis 


\section{CONTENTS}

Chapter 1 Introduction 9

1.1 Pain and pain perception 9

$\begin{array}{lll}1.2 & \text { Chronic pain } & 12\end{array}$

$\begin{array}{ll}\text { 1.3 Chronic low back pain } & 13\end{array}$

Chapter 2 Psychophysiological models of chronic pain 15

2.1 Introduction 15

2.2 Reflex-spasm model 15

$\begin{array}{lll}2.3 & \text { Stress-reaction model } & 17\end{array}$

2.4 Biomechanical model 20

$2.5 \quad$ Miscellaneous physiological hypotheses 21

2.6 Discordance and pain disorders 22

2.7 Methodological considerations of psychophysiological

$\begin{array}{lll}2.8 & \text { Conclusion } & 26\end{array}$

$\begin{array}{lll}2.9 & \text { Questions addressed in this dissertation } & 27\end{array}$

Chapter 3 Chronic pain and experimental pain sensitivity 29

$\begin{array}{lll}3.1 & \text { Introduction } & 29\end{array}$

$\begin{array}{lll}3.2 & \text { Hypochondriasis } & 29\end{array}$

$3.3 \quad$ Learning processes and stimulus generalization 33

3.4 Adaptation Level Theory 34

3.5 Activity of endogenous opioids $\quad 35$

$\begin{array}{lll}3.6 & \text { Counterirritation and DNIC } & 38\end{array}$

3.7 Review of studies assessing sensitivity for experimental 43

3.8 A hypothetical model of pain responsiveness 48

3.9 Sensitivity for non-painful interoceptive and tactual signals 52

3.10 Questions addressed in this dissertation 53

\section{Chapter 4 Chronic pain and habituation to} experimental pain stimuli $\quad 57$

4.1 Introduction $\quad 57$

4.2 Models of habituation $\quad 57$

4.3 Habituation to experimental pain stimuli 63

4.4 Factors influencing habituation to pain stimuli 64

4.5 Chronic pain patients and differences in habituation to
experimental pain stimuli

4.6 Questions addressed in this dissertation 69 
5.1 Chronic Low Back Pain and the reaction to repeated acute pain stimulation

5.2 Psychophysiological responses to repeated acute pain stimulation in chronic low back pain patients

5.3 Differences between CLBP patients and controls in the perception of experimental pain at various intensities

5.4 Is there evidence for cognitive-physiological discordance and desyrichrony in CLBP patients?

5.5 A comparison of two-point discrimination threshold of tactual, non-painful stimuli between chronic low back pain patients and controls

5.6 The influence of a counter-irritation stimulus on pain perception and pain discrimination

5.7 Differences in pain perception and sensory discrimination between chronic low back pain patients and healthy controls

5.8 Suppression of the nociceptive flexion reflex with two different counter-irritation stimuli

5.9 Chronic back pain, acute postoperative pain and the activation of diffuse noxious inhibitory controls (DNIC)

Chapter 6 Discussion

6.1

Psychophysiological models of chronic pain

6.2

Chronic pain and experimental pain sensitivity

6.3

Chronic pain and habituation to experimental pain stimuli

6.4

Concluding remarks

Summary

Samenvatting

References

List of Abbreviations

Dankwoord 


\section{INTRODUCTION}

This dissertation is about chronic pain and pain perception. Several different approaches to this broad subject will be presented, most of them being related to one of two major questions. These questions are: (1) why do chronic pain patients perceive pain when there is no identified pathology (i.e. Where does this pain arise from), and (2) do chronic pain patients have a different perception of acute (experimental) pain than pain-free people? To begin with the first topic, in a substantial proportion of the people complaining of chronic pain, no somatic cause for the pain can be established. In chapter two of this thesis, psychophysiological models of chronic pain will be discussed which might partly explain some of these pains. These models propose that psychological and behavioural factors can induce physiological changes which ultimately might be responsible for the generation and maintenance of pain. The second topic is addressed in chapters 3 and 4 . In chapter three the question of whether chronic pain patients have a different perception of (experimental) pain stimuli will be considered. Several hypotheses which predict such differences in pain perception will be discussed. Chapter four adresses the question of differences in pain perception between chronic pain patients and healthy subjects in another way, by looking at the perception of repeated pain stimuli. The question in this chapter is whether habituation to pain stimuli is disturbed in chronic pain patients.

However, before going into the various theories of chronic pain and pain perception in the next three chapters, in this introductory chapter an attempt will be made to clarify the conceptualizations of pain, pain perception, and chronic pain as they are employed in this dissertation.

\subsection{Pain and pain perception}

The question of what pain is, is not easy to answer and numerous definitions of pain have been advanced. In older views, pain was seen as a sensory experience dependent solely on the quality and intensity of noxious stimulation and the extent of tissue damage. This view has dominated western thinking on pain for a long time. Not until a few decades ago was it recognized that there is more to the experience of pain than the uncomplicated and uninterrupted transmission of a noxious signal to the brain. Still, the fact that sensory factors play an important role in the experience of pain goes unchallenged. In brief, pain usually begins with the occurrence of tissue damage. This, in turn, initiates a series of electrical and chemical physiological events, which together constitute the process of nociception. Nociception consists of the sensory detection of the noxious stimulus by the nociceptors, the transduction of this stimulus from a chemical into an electrical signal, and subsequently the neuronal transmission of this signal. Only when the nociceptive activity is detected and consciously experienced, the term "pain" should be applied. 
The formulation of the gate-control theory in 1965 (Melzack \& Wall, 1965) has had an enormous impact on modern thinking about pain. This model emphasizes the influence of spinal cord and brain processes on the experience of pain. These processes can modulate the relationship between noxious input and final pain experience. That there is not a one to one relationship between the intensity of the noxious input and pain perception is shown by several observations. Although nociceptive stimulation usually forms the basis of pain perception, it is not in itself a sufficient condition for pain to occur, and probably it is not even a necessary condition. Nociception without the perception of pain can occur for instance in circumstances of extreme emotion and stress. Famous are the stories of soldiers wounded on the battlefield, who are not aware of any pain sensation, despite considerable injuries, until much later when they are in safety (Beecher, 1959). Much less extreme, but more common, is the observation that during exciting sports matches players sometimes do not feel an injury sustained during the match. Alternativelly, pain without nociceptive input also seems to exist. The absence of physical findings in someone complaining of pain is in itself not sufficient to assume that no nociception is present. Nevertheless, the DSM-III-R (American Psychiatric Association, 1987) describes at least one pain condition in which nociception apparently does not play a role: the somatoform pain disonder. In addition, in rare circumstances, hallucinatory states and schizophrenic conditions can be accompanied by pain.

Obviously then, there is no simple relationship between noxious stimulation and pain perception. Recent neurophysiological insights have revealed that nociceptive activity can be modulated at different nervous system levels. Powerful inhibitory influences have been found, but also processes enhancing nociceptive activity (Fields, 1987). Psychological processes can be potent activators of such modulating processes. It has already been mentioned that emotions and stress can decrease the perception of pain, and this seems to be at least partly dependent on its direct effect on spinal nociceptive transmission. A number of other factors were found to modulate the experience of pain, either through the modulation of nociceptive transmission or through an effect on the transition of the nociceptive activity to the conscious experience of pain. In this respect personality factors, attentional and cognitive states, and social and cultural factors should be considered. Therefore, if pain is described as a sensory experience, at least it should be acknowledged that it is a complex sensory experience, amenable to modulation.

The current defimition of pain employed by the International Association for the Sudy of Pain (IASP, 1979) is as follows: "An unpleasant sensory and emotional experience associated with actual or potential tissue damage, or described in terms of such damage". "Two points are of interest in this definition. Firstly, it tells us that pain can occur in the absence of actual tissue damage, and secondly, it stresses the fact that pain is not only a sensory experience, but an emotional experience as well. Pain is associaled with negative affect, it is usually experienced as aversive, upsetting or disturbing.

The approach to pain perception in this dissertation is based on the notion of a multifactorial determination. Modulation of nociception and the experience of pain 
can take place at different levels: in the periphery (e.g. alteration of nociceptor sensitrvity), at spinal sites (segmental and inter-segmental modulations), in the brainstem (e.g. descending inhibitions) and at higher brain/cortical levels (e.g.cognitive and attentional influences, social factors, learning, etc.). This wiew of pain perception is summarized in figure 1.1 , which is adapted from a model constructed by Melzack \& Loeser (1978) to explain phantom limb pain. Phantom limb pain is another condition which is not explicable in terms of peripheral nociceptive input. In this condition, pain is felt in an amputated limb. Obviously, the sensation cannot arise from nociceptive sensory input from the limb itself. Spinal and central mechanisms are held responsible for the generation of pain.

\section{TO BRAIN AREAS THAT UNDERLIE PAIN EXPERIENCE}

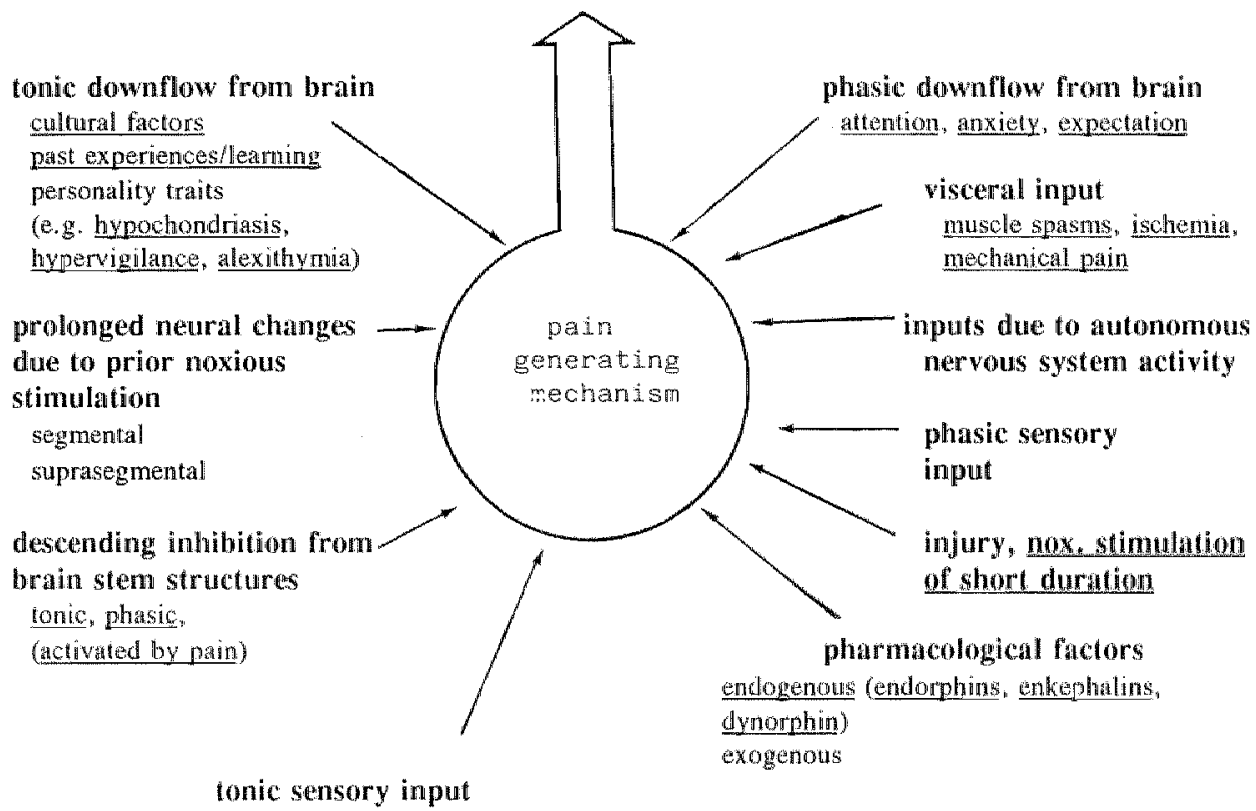

trigger points, scar tissue

Fig. 1.1 A model of pain perception in which the final conscious experience of pain is determined by the joint influences of the various factors. The concepts in the model which are underlined will be addressed in subsequent chapters. 
To determine the influence of some of the factors in the model it is necessary to establish the intensity of pain someone is experiencing. But pain is a private experience, not accessible to direct assessment. Therefore, one has to rely on the (verbal) communication and expression of pain, which is not necessarily a reliable reflection of the actual pain experience. Verbal and behavioural expression of pain are under environmental control. Fordyce (1979) has repeatedly stressed the importance of social contingencies in the shaping of pain responses. Also, personality and cultural variables will influence pain expression. On the other hand, we do not have any other means of knowing the pain experience other than by asking a subject. That is why in this dissertation the communication of pain intensity will be equated with actual pain perception.

\subsection{Chronic pain}

Just as there are different definitions of pain, chronic pain too has been defined in several ways. There is no consensus about what should be classified under the heading of chronic pain (see for instance the recent discussion in the paper "What is chronic pain?", The Clinical Journal of Pain, 6, 1990, 160-166). Most authors agree that the duration of pain should be included in the definition. Usually, a criterium of 6 months is applied, and when pain exceeds this duration it is called chronic. Some authors have argued that this criterium should be lowered, for instance to two months, because by that time the chance of recuperation from the pain has decreased dramatically (Schmidt, 1986). Sometimes the definition of chronic pain contains the stipulation of no physical findings and no identified nociceptive process (American Medical Association, 1988). In this case only painful conditions without an apparent physical cause are considered as chronic pain. However, more generally, chronic pain is defined independently from whether or not physical findings are present. This last view is for instance representative of the approach of Chapman \& Bonica (1985). They divide chronic pain into 3 categories: (1) pain that persists beyond the normal healing time for an acute injury or disease, (2) pain related to a chronic disease or persisting neurological condition and (3) pain that emerges and persists for months or years without identifiable organic cause. Thus, chronic pain in this view may or may not have an identified physical cause. Another distinction which is sometimes made is between chronic benign pain and chronic malignant pain. The latter condition refers to a degenerative disease, leading to physical deterioration and which is potentially life-threatening. Benign pain, on the other hand, is not associated with a degenerative disease, and it is not life-threatening. Nevertheless, "benign" pain can be very disabling.

All pain complaints which exist for longer periods of time (whether malignant or benign, with or without identified cause) have several features in common. For instance, there is usually a considerable impact of the pain on the psychological functioning of a patient, independent of the etiology of pain. Persistent pain is associated with changes in mood states (depression, frustration, feelings of helplessness), sleeping problems and substance abuse. Moreover, psychological and 
social factors contribute to the pain experience and suffering in all pain patients, even in patients with acute pain. Patients with identical diseases or identical injuries may vary widely as to the degree of pain they perceive. In sum, every pain, acute as well as chronic, can be considered to be the result of a combination of physiological, psychological and social/environmental processes.

This approach to chronic and acute pain also assumes that delineation of organic pains as opposed to psychogenic pains is unfruitul. There is no pure organic pain, just as there is no pure psychogenic pain. Even when the inwolvement of organic pathological processes is straightforward, as in cancer pain or in rheumatoid arthritis, psychological and social factors contribute to the pain experience. The other way round, if a pathophysiological cause is not directly obvious, and psychological factors seem to be implicated, a somatic contribution to the pain problem can be present. For instance, inactivity caused by the persistence of pain could lead to a deterioration of physical condition, which may worsen the pain complaint. Moreover, the warious influences on the experience of pain are not mutually independent. Psychological. processes, for instance, can also induce physiological dysfunctions (which in tum can lead to more psychological distress, more dysfunction, etc.). It has been hypothesized that such dysfunctional somatic changes may be especially important in the development and maintenance of certain chronic pain conditions with minimal pathological findings like chronic low back pain (CLBP) and headaches. Psychophysiological research into these dysfunctional physiological factors has predominantly focussed on abnormal muscular and autonomic functioning. In chapter 3, three different psychophysiological models are discussed. Of course, in agreement with the theorizing above, even if a relevant psychophysiological factor is identified, it does not exclude the influence of other factors on the total chronic pain experience.

\subsection{Chronic Low Back Pain}

Although the three theoretical chapters which are to follow are concerned with chronic pain in general, the experimental studies have only involved CLBP patients. Chronic low back pain is a typical chronic pain syndrome, with multiple factors determining the pain experience and disability of the patient. CLBP is also at widespread problem, affecting large numbers of people, and it is a major cause of disability. In the Netherlands, disorders of the locomotor system are the most common cause of benefit according to Dutch disability insurance. Moreover, the percentage of this disorder of total disability benefits is increasing (from $26 \%$ in 1985 to $26.7 \%, 27.2 \%, 27.7 \%$ and $28.3 \%$ in $1986,1987,1988$ and 1989 respectively, figures according to the Dutch Communal Medical Service, GMD). Although the specific contribution of CLBP in this total percentage is not known, it is probably of major importance.

In the majority of patients with CLBP, no physical cause has been identified. Percentages of 70 (Vallfors, 1985) and 75 percent (Chapman \& Bonica, 1985) of the total CLBP population have been reported to have back pain with unknown physical cause. Pain in the back is a symptom which can be associated with a variety of 
somatic diseases: disc prolapses, inflammation of the meninges, cancer, ankylosing spondylitis, spinal infections, etc. These conditions have been excluded in the patients participating in the studies described in chapter 5 . None of these patients had an identified somatic disease. Whether the results of these experiments with CLBP patients can be generalized to other chronic pain syndromes should be tested further. 


\section{PSYCHOPHYSIOLOGICAL MODELS OF CHRONIC PAIN}

\subsection{Introduction}

In the previous chapter it was argued that chronic pain without an explained physical cause may be associated with dysfunctional somatic processes, brought about, maintained or exacerbated by behavioural or psychological factors. In this chapter, various models of benign chronic pain based on such dysfunctional processes will be reviewed. The term psychophysiological models of chronic pain is used to describe these models.

Most studies which look for (psycho)physiological correlates of chronic pain consider these correlates to be of etiological significance. Several of such etiological psychophysiological models have been described in the literature. The three main models are the reflex-spasm model, the stress-reaction model and the biomechanical model. The terminology used to refer to these models has not always been consistent. The reflex-spasm model has allso been referred to as the physical stressor model (Dolce \& Raczynski, 1985), and the stress-reaction model has been described under the names stress-causality model (Nouwen \& Bush, 1984) and psychosocial stressor modell (Dolce \& Raczynski, 1985).

Several predictions based upon the three models have been tested in various pain syndromes. The two syndromes most extensively studied in relation to a psychophysiological etiology have been headache and back pain. How the models pertain to these two syndromes and the results of the studies testing the predictions arising from the modells will be discussed. Recently, Flor \& Turk (1989) have written an extensive review addressing the relationship between psychophysiological variables and chronic pain. They included 47 headache relevant studies and 8 CLBP relevant studies in this. review. Their main conclusions are incorporated in the present overview. However; they did not relate their conclusions to the three models described above and also ignored the biomechanical model. In addition, some new studies, published after the sampling of Flor and Turk was finished, have been included in the present overview.

\subsection{Reflex-spasm model}

The reflex-spasm model is a so-called myogenic pain model, which means that muscle tension is assumed to be the cause of pain. The model states that initially an organic pathology or lesion is present, which will give rise to a muscle-spasm reflex in the affected area. Local muscle spasms after pain have been shown to occur in the cat as well as in man. In an experiment using cats, Pederson et al. (1956) demonstrated that mechanical pain applied to either spinal joints, fascia, ligaments, 
muscle tissue or the skin overlying the spine, was followed by reflexive spasms of the erector spinae, hamstrings and other muscles. These spasms often continued for a while after the pain stimulus. Lewis \& Kellgren (1939) have reported similar findings in both cats and men, after the injection of hypertonic saline into interspinous ligaments.

Muscle spasms are believed to be beneficial in the case of lesion, because further damage to the surrounding tissue is prevented by immobilization of the area. Cailliet (1981) describes how spinal joint inflammation leads to periarticular (=around the joint) muscle spasms and concomitant immobilization of the inflamed joint, which is undoubtedly adaptive in the acute pluase of inflammation. However, when muscle contractions are sustained, they can themselves become a source of pain. Two mechanisms might be responsible for this. First, muscle spasms can prevent adequate blood circulation through the affected area and give rise to an ischemic condition. By the accumulation of waste products, muscular pain can subsequently develop (Wyke, 1987). Alternatively, muscle spasms could cause pain through irritation of the joint and related structures by compression of an already degenerated disc. If muscle spasms result in pain, this pain can in turn lead to even more muscle spasms. In this way, a vicious circle of pain-spasm-pain develops. (Hendler, 1981; Nouwen \& Bush, 1984 ).

The reflex-spasm model has been applied to various pain syndromes, among which are CLBP and tension headache. In the case of tension headache, it is hypothesized that an initial neck or head injury elicits protective muscle spasms in the neck and shoulder region, which ultimately results in pain. Therefore, it is predicted that patients with tension headache show sustained increased tension of neck, shoulder or facial nuscles. Various studies did indeed find tonically increased muscle tension in the neck and head region (e.g. Sturgis et al., 1984). Often the frontal muscles were involwed. However, the evidence does not seem to be overwhelming and a considerable number of negative studies have been reported as well. Flor \& Turk (1989) argue that when taking the quality of the studies into consideration, the majority of what they considered to be the "best" studies did not find tonic physiological differences between headache patients and healthy controls. They conclude: "Overall, the results on recurrent headache argue against the simple hypothesis of permanently changed physiological processes such as elevated EMG levels (...) in patients with charonic headaches". However, it has also been proposed that differences in muscle tension are not permanent, but only apparent during headache states, while absent during pain-free periods. A very recent study has not been able to confirm this prediction. Tension levels of the frontalis and trapezius muscle were the same during headache attacks and during headlache-free periods. Howewer, in the same study some confirmation was found for another suggestion, namely that differences in muscle tension between headache patients and controls might be position dependent. Tension headache patients were tested in six different positions, and in one of them (the prone position), both trapezius and frontalis muscle tension was higher (Arena et. al, 1991).

For CLBP, the reflex-spasm model assumes an identical etiological mechanism: an initial acute back injury or organic pathology gives rise to protective muscle contrac- 
tions in the back. As the pain-spasm-pain circle develops these muscle contractions will be sustained and a tonically increased tension in the back musculature is established. Thus, sustained increases in paraspinal muscles tension are predicted in CLBP. A tonic increase in paraspinal muscle tonus could be confirmed in some studies. However, other studies did not find indications of increased muscle tonus in the paraspinal muscles of CLBP patients Nouwen \& Bush, 1984 ; Feuerstein et al., 1987; Amtz et al., 1991). Just as in headache research, it has been suggested that differences in muscle tension might be posture related. In standing patients, higher, but also lower EMG amplitudes for the paraspinal muscles have been found. During movement, there have also been findings of increased, as well as decreased or similar activity in CLBP patients in comparison to a control group (Feuerstein et al., 1987; Hoyt et al., 1981; Nouwen \& Bush, 1984; Soderberg \& Barr, 1983; Arena et al., 199 (b). Whether pain state affects the results was also lested for CLBP patients. EMG recordings of forty-six CLBP patients were compared to the recordings of normal controls in six different positions. Every patient was measured twice, once while they experienced a low to normal pain level, and once when they experienced a normal to high pain level. No overall effect of pain level was found. However, looking specifically at the EMG of the "most abnormal position", i,e. the position in which the largest deviation from control EMG levels was found for a particular individual, then pain level proved to be a significant factor (Arena et. al, $199 \mathrm{lb}$ ).

All in all, the reflex-spasm model found only partial confirmation in both tension headache and CLBP studies. The model does not seem to be capable of explaining the majority of chronic pain complaints. In no way does increased muscle tension appear to be continuously present. Some indications have been found for temporarily increased muscle tension, related to certain postures and movements.

\subsection{Stress-reaction model}

The second model, the stress-reaction model, assumes two necessary conditions for the development of chronic pain: exposure to some kind of aversive stimulation which is experienced as stressful, and the existence of inadequate homeostatic control. The latter means that there is an abnormally large physiological reaction to stress or that the recovery from the physiological stress reaction is retarded. A central rolle in this model is played by the concept of response stereotypy. This means that the pattern of physiological responses is specific for an individual, he/she will always react to stressors in the same way physiologically. The existence of individual response stereotypy was convincingly demonstrated by Robinson et al. (1987). A possible consequence of such a stereotyped way of reacting is that frequent activation of the same response system (which can be any response system) can eventually lead to a breakdown in this system. Thus, which type of complaint will develop depends on which system is most responsive to stress. The nature of the stressor can be very diverse, but for breakdown to occur the stressful condition has to occur repeatedly or be continuous. If breakdown occurs, one of the consequences could be the develop ment of pain. Pain itself can become a new stressor, and thereby maintain itself, just 
as in the previous model (Nouwen \& Bush, 1984).

The stress-reaction model has been applied to migraine, tension headache and CLBP. The model argues that in non-stressfull conditions, no systematic differences in physiological activity between chronic pain patients and controls will be found. However, during stress, differences in reactivity become apparent. This increased physiological stress responsiveness can be expressed in any response system, but will probably be most pronounced in the specific response system related to a particular pain syndrome. For migraine patients this means that in particular increased vascular responses to stress are predicted. Migraine is usually seen as a vascularly based headache. For tension headache patients it is predicted that increased stress responsiveness occurs in head, neck and shoulder muscles. Finally, CLBP patients will probably show the greatest stress responsiveness in the back musculature. However, this does not exclude that a wider range of autonomic stress responses are also more pronounced in these patients compared to pain-free controls.

Flor, Turk and Birbauer (1985) on the other hand, have suggested that increased stress responsiveness is confined only to the specific response system related to the complaint. They have elaborated the stress reaction model into a more specific model, in which they emphasize the specificity of the physiological reactivity. They have applied their so-called diathesis-stress model in particular to CLBP. They have argued that CLBP can develop when: (1) a response stereotypy (diathesis) exists, involving the back muscles (this may be due to injury, predisposition, etc.), (2) aversive stressful events are present and (3) an individual has inadequate coping abilities. The mechanism by which pain will ultimately arise has also been described: repeated or sustained muscle tension causes ischemia and subsequently the release of pain-eliciting substances (e.g. bradykinin) (Turk \& Flor, 1984). The pain elicited by this process can itself act as a new stressor, thereby starting a vicious circle. The resemblance of the mechanism of this model to that of the reflex-spasm model is obvious. However, the initial cause of the muscle spasms differs (stress warsus physical injury).

The predictions of the stress-reaction model (and the diathesis stress model) have been tested empirically. In tension headache patients, increased stress responses in the head (frontalis, occipitalis and temporalis) and neck muscles have been found (Pritchard \& Wood, 1983; Thompson \& Adams, 1984). An abnormal vascular response to stress was found in migraine patients (Bakal \& Kaganov, 1977; Arena et al., 1985; Rojahn \& Gerhards, 1986). This is revealed by greater cephalic vasoconstriction (allhough sometimes it is the cephalic vasodilatatory response which appears to differ), a stronger digital vasoconstriction and an unusual large heart rate acceleration. Furthermore, migraine patients have been found to show slower habituation of these stress responses (Morley, 1985), as well as a delay in return to baseline (Arena et al., 1985; Gannon et al., 1981). In addition to an increased vascular stress response, also an increased muscular response was found in migraine patients (van Olst et al., 1980; Pritchard \& Wood, 1983). However, here too, some of the studies failed to demonstrate any difference between migraine and muscle tension headache patients on the one hand and controls on the other (Feuerstein et al., 1982 ; KronerHerwig et al., 1988). Perhaps this can be partly attributed to the types of laboratory stressors used (Passchier, 1987). In most instances, these stressors show little 
resemblance to daily life stressors. The laboratory stressors are usually of short duration and do not involve the subject emotionally.

Another problem is the cause - effect relationship between headache and increased physiological stress responsivity. The stress-reaction model is an etiological model, and assumes that the increased stress responsivity is responsible for inducing headache. However, it has also been found that increased muscular and vascular responsivity only occurred when headache was present at the moment of testing. Therefore, physiological differences in headache patients may be the consequence of their headache, rather than the cause (Hursey et al., 1985; Haynes et al., 1983; Bakke et al., 1982). As has been discussed in the previous section, headache state did not affect tonic muscle tension level. However, it might influence the stress responsivity.

In a study by Gannon et al. (1987) an attempt was made to make inferences about the direction of causality between physiological responsivity and headache. In addition, these researchers tried to avoid the problem of a stressor of too short a duration. A one-hour cognitive stressor task was presented, and multiple electromyographic and cardiovascular measures were recorded in migraine patients, tension headache patients and controls. The most important finding was that the stressor induced a headache in the majority of headache subjects but in only few control subjects, and that this headache was preceded by an increased cardiovascular and muscular activation. In contrast to earlier studies, not only specific muscular and vascular variables were related to headache, but also more general physiological parameters (heart rate and forearm EMG).

In agreement with the present review, Flor and Turk in their review conclude, again considering the quality of the studies, that: "there is some evidence for symptom-specific responding in patients suffering from chronic headaches. Although baseline resting levels do not differ between headache patients and healthy controls, a number of studies, especially those that used adequate designs and long and relevant stress exposure, found differences in responses to stress in pericranial EMG levels and several vascular measures."

A number of studies have tested the predictions arising from the stress-reaction model in CLBP patients. Holmes \& Wolff (1952) were the first to report on the role of physiological stress responses in the etiology of CLBP, by reporting increased tension levels in the skeleton muscles during stressful interviews. Their single subject observations led to further investigations. These later studies, in general, have been unable to replicate the originall findings (Feuerstein et al, 1987; Wilfling, 1981). Some support has been found for an increased frontalis EMG response to experimental stress in CLBP patients (Collins et al., 1982; Nouwen \& Bush, 1984). However, there is little evidence of increased reactivity in other muscles or in the autonomic nervous system.

Like in headache research, in studies with CLBP the need for relevant stressors has been emphasized. Flor et al. (1985) have suggested that previous failures to find increased stress reactivity in CLBP patients could be due to the fact that most studies 
until that time had made use of experimental stressors with little relevance to the subjects. One of the premises of their diathesis stress model is that an individual experiences a stressor as aversive. Moreover, their model predicts a specific response in the paraspinal muscles exclusively. In testing the diathesis stress model, Flor et. al. compared the physiological reaction of CLBP patients to non-relevant and relevant stressors. The relevant stressor consisted of the discussion of an aversive emotional situation, the irrelevant stressor was a memtal arithmetic task. Only in response to the relevant stress condition, an increased reaction in the paraspinal muscles was found in the patient group. Non-specific autonomic and frontalis EMG-responses were not different for patients and controls.

The hypothesis of increased specific physiological stress-responsivity in CLBP patients has also been tested in our laboratory (Arntz et al., 1991). Repeated painful electrical shocks were used as the stressor. Pain can be supposed to be a relevant stressor for chronic pain patients. An increased responsiveness in the paraspinal muscles was found for CLBP patients after electrical shocks. There appeared to be specificity in this increased responsiveness, general autonomic reactivity (skin conductance, finger pulse volume and heart rate) was not higher in the patient group. However, the frontalis muscle response was also elevated for CLBP patients, but this might have been due to the lower tonic frontalis EMG levels of the patients ("law of initial values").

In sum, the diathesis-stress model of CLBP seems to be supported empirically, by at least the two studies reported here. Replication of this results would be welcomed.

In conclusion, evidence for the stress-reaction model seems to be stronger than for the reflex-spasm model. Both for headache and CLBP confirmation for the model could be found, but the relevance of the stressor which was used in the studies appears to be an important mediating variable. The evidence for increased reactivity in specific response systems is superior to that for general increases in physiological responsiveness to stress.

\subsection{Hiomechanical model}

The most recent etiological model of chronic pain is the biomechanical model. It was especially formulated for explaining the development and maintenance of chronic back pain. The biomechanical model hypothesizes that during the acute pain plase (e.g. resulting from an injury) patients develop postural abnormalities as a reaction to the pain. The faulty patterns of muscular activity resulting from these postural abnormalities, in turn, affect the biomechanics of movement (Dolce \& Raczynski, 1985). The spine becomes unstable by asymmetrical or abnormally low levels of muscle activity which can eventually result in mechanically induced pain. Thus, in contrast to the two models discussed above, this is not a myogenic pain model: the ultimate catse of pain is not muscular but mechanical, resulting from nerve root, joint or capsular irritation.

The biomechanical model thus predicts that CLBP patients have abnormally low 
levells of tension, left-right asymmetries or aberrant patterns of activation in the back nuscles. There have been a number of studies testing these predictions (Nouwen \& Bush, 1984; Nouwen et al., 1987). Overactivity in some muscles, with accompanying underactivity in others has been found. Furthermore, asymmetrical reactions have been shown to occur in back pain patients, where symmetrical activity was expected, while another study found greater discrepancies between the activity of the muscles on the right and left side in CLBP patients compared to controls (Cram \& Steger, 1983). As predicted by the biomechanical model, the regulation of the muscle activity seems to be affected: in rest, patients are unable to relax the muscles sufficiently, and in tasks with great demands on muscle activity, patients do not contract sufficiently. It has also been found that patients activate more muscles than controls do in performing the same task. And finally, it has been found that patients are unable to relax the back muscles while contracting other muscles (Kravitz et al., 1981).

Thus, support has been found for the biomechanical model of chronic back pain. Nevertheless, some reservations should be noted pertaining to the model. The biomechanical model does not predict specifically what muscular changes will occur in back pain patients, and it can therefore accommodate a wide variety of lindings. Almost any distinction between back pain patients and controls on muscular variables can be considered meaningful within the framework of the model. Moreover, all studies directed at this model so far, have been correlational in nature, and whether differences in muscular patterns are an antecedent or a consequent factor of chronic pain is not clarified. A parallel can be drawn with personality measurements in chronic pain patients: all kinds of differences have been found between pain patients and controls, but the causal role of these correlates remains obscure. One last critical remark can be made concerning the fact that only one component (muscular patterns) of the proposed mechanisms is tested. Are the muscular abnormalities indeed caused by postural aberrations, and do these muscular abnormalities in turn generate mechanical pain? A major part of the sequence of events remains speculative. (This last criticism is not specific for the biomechanical model. Also in the reflex-spasm model for instance, only increased muscle tension is tested, regardless of whether this subsequently leads to ischemia or joint irritation, which are ultimately supposed to generate pain).

Still, the biomechanical model offers some interesting possibilities for further research. To get more insight in the causal relationships between the processes proposed in the model, longitudinal studies are required instead of cross-sectional ones. For instance, it could be tested whether the formation of postural abnormalities during an acute back pain episode predicts chronicity. On the other hand, physical therapy aimed at the correction of faulty postures should prevent the development of chronic pain. Such studies would obviously have major clinical implications.

\subsection{Miscellaneous physiological hypotheses}

In addition to the three models described above, there have been various other hypotheses assuming sustained physiological differences between chronic pain patients 
and controls. These hypotheses are discussed separately as they do not fit in with any of the three models described above. One such hypothesis relates to migraine. Migraine has been assumed to have a vascular basis, and the presence of sustained vascular abnormalities have been hypothesized. Morley (1985) did indeed demonstrate such tonic vascular abnormalities in migraine patients, namely a greater variability in the temporal artery pulse amplitude. Moreover, the finding of a vascular dysfunction has not been restricted to migraine, but could also be found in tension headache patients (Philips \& Hunter, 1982). Therefore, the strict separation of migraine as vascularly based and tension headache as muscularly based seems to be contradicted empirically. Muscular dysfunctions comparable to those found in tension headache patients (see reflex-spasm model), have also been found in migraine patients (Bakal \& Kaganov, 1977).

Other miscellaneous hypotheses have speculated on generalized autonomic and muscular hyperactivity, not related to stress. From a review of the relevant studies, it is apparent that there is little empirical support for a general sustained physiological hyperactivity, either in headache or CLBP patients (Feuerstein et al., 1987; Nouwen \& Bush, 1984).

\subsection{Discordance and pain disorders}

Another etiological model of pain disorders with psychophysiological aspects is based upon the concept of discordance. Concordance and discordance relate to the degree of agreement or disagreement between measures of two or more response systems assumed to reflect the same process. Gannon \& Haynes (1986) have proposed that discordance can contribute to the development and maintenance of several psychophysiological disorders, among which headache, insomnia and sexual disorders. They have applied discordance especially to differences between the cognitive and physiological response system, and use the term cognitive-physiological discordance. In this model it is proposed that discordance will be particularly evident for the physiological response system underlying a specific disorder. For chronic pain patients this implies that discordance between the subjective experience of pain and objective correlates of the presumed physiological basis of the pain (e.g. muscle tension or vascular dysfunction) is apparent.

Gannon and Haynes also speculate on why and how discordance can llead to physiological disorders. Cognitive-physiological discordance is considered to be an expression of the subject's non-attendance to proprioceptive cues. Inattention to internal signals, which can be caused by an inability to perceive such signals, or by increased distractibility, prevents the initiation of appropriate coping behaviours. In turn, this will lead to inadequate self-regulation, and eventually to a dysfunction. Also, the unawareness of causal relationships among external events and symptoms of the disorder will prevent the initiation of coping strategies.

Research aimed at demonstrating physiological-cognitive discordance in chronic pain patients are hampered by many difficulties, and evidence for this kind of discordance, let alone for an etiological significance of it, is as yet scarce. One major 
problem in such studies is that it is rarely apparent which physiological variable should be measured. In the previous section it was described that most physiological correlates of chronic pain are still speculative. Thus, if it is found that the pain reports of tension headache patients do not correlate with objectively quantified frontalis muscles tension, do these patients display discordance, or has the wrong physiological variable been measured? Another problem in assessing discordance between two different response systems is method variance. The degree of correlation between variables which have been measured by different methods is always limited. Physiological variables will usually be measured electrophysiologically, and cognitive variables by means of self-report. This can result in apparent discordance. For an extensive review of these and other statistical and procedural sources of apparent discordance the article by Gannon and Haynes should be consulted.

Based upon the three system model of emotions (Lang, 1968), which states that emotions can be expressed through the overt-motoric system, through the physiological system and through the verbal-cognitive system, Vlaeyen (1991) argues that discordance can be seen between any of these systems. This would mean that three types of discordance can be distinguished: in addition to the cognitive-physiological discordance, also cognitive-behavioural and behavioural-physiological discordance. Thus far, only for cognitive-behavioural discordance ample evidence has been found in chronic pain patients. Indeed, it is a common clinical. observation that in patients with chronic pain, the verbal indications of pain and the observable pain behaviours often disagree. Vlaeyen has reviewed several studies in which non-verbal pain behaviours and pain ratings in chronic pain patients were measured. He reports that usually low correlations are found between the two. However, the significance of cognitive-behavioural discordance as a cause of chronic pain is doubtful. Rather it can be regarded as an outcome of several different processes which are active in chronic pain. Pain behaviour is considered to be under consequent control, shaped by operant learning processes (Fordyce, 1983). The cognitive aspect of the pain experience is not necessarily under the control of the same process or the same contingencies. For behavioural-physiological discordance a more apparent etiological role can be envisaged, in analogy of cognitive-physiological discordance. However, as has been discussed with cognitive-physiological discordance, testing of behavioural-physiological discordance is problematic, because of the difficulty of selecting (clinically) relevant physiological parameters.

The definition given of discordance at the beginning of this section was "a disagreement between measures related to different response systems"'. However, one can also assess the agreement between two different measures related to the same response system. Disagreement within the cognitive system was found by Perry et all. (1988). They compared the scores on a VAS pain rating scale with the scores on several subscales of the McGill Pain Questionnaire (MPQ, subscales: present pain intensity, sensory, affective and total pain rating index) in patients with demonstrable tissue pathology (arthritis) and patients with litte or no demonstrable tissue pathology (myofascial pain). Correlations among the pain measures were consistently found to 
be higher and less variable in arthritis patients than in myofascial pain patients. Thus, the cognituve pain variables were more "concordant" in arthritis patients than in myofascial pain patients, although, in fact, one cannot speak of concondance/dis. cordance according to the definition presented above.

Related to the original model of cognitive-physiological discordance as formulated by Gannon and Haynes, is the concept of alexithymia. Alexithymia literally means "no words for feelings" and denotes the inability to express emotions verbally. Often the concept is used in a broader sense, implying that persons who display alexithymia are not only incapable of the verbal expression of emotions, but that they are actually unaware of their own emotions. Thus, there is supposed to be a decoupling between the emotional experience of stress, indicated by subjective report of tension, and the physiological response to stress (Papciak et al., 1985; Martin \& Phil, 1986). Flannery (1977) has hypothesized that alexithymic people are more likely to develop psychosomatic illness because of increased muscle tension and autonomic reactivity resulting from unexpressed emotions. Similarly, Feuerstein et al. (1987) stated that the decoupling of cognitive and physiological stress responses can result in recurrent or continued autonomic arousal and thereby an increased chance of deweloping somatic symptoms. Martin and Phil have speculated that the presence of alexithymic characteristics prevents an individual from coping effectively with a stressor. This is due to the unawareness of this person of the presence of stress and arousal (Martin \& Phil, 1985). Or in the words of Feuerstein et al:: "it is hypothesized that recurrent or chronic arousal is associated with an inability to self-manage environmental stressors due to a lack of awareness of such stressors". The resemblance of these formulations to the cognitive-physiological discondance model of psychosomatic complaints is obvious: the emphasis is on lack of awareness of and inadequate coping in response to internal signals (discordance) or external stressors (alexithymia).

Alexithymia can in this way be characterized as a risk factor for developing chronic pain. Feuerstein et. al. have noted that a relatively high percentage of chronic pain patients indeed do display alexithymic characteristics. Research has suggested that alexithymia is more common in chronic pain patients than in people with other medical conditions or patients undergoing psychotherapy (Mendelsohn, 1982; Postone, 1986). To make the association between alexithymia and chronic pain even stronger, a direct relationslup has been found between emotional expressivity (as measured by an allexithymia scale) and pain duration and interference of pain with daily activity in cancer pain patients. As emotional expressivity decreased, pain duration and interference increased. For non-cancer pain patients, a significant negative relationship was found for alexidymia and pain interference only (Dalton \& Feuerstein, 1989). However, a recent study by James \& Large (1991) has shown that

In a recent study (Perry et al. 1991) this finding could be replicated in patients with gastro-intestinal pain syndromes, but only for the correlation between MPO present pain intensity and VAS pain score. These two variables correlated significantly in patients with (organic) inflammatory bowel disease, but not in patients with the irritable bowel syndrome (presumed to be a functional complaint). 
allexithymia as measured by three different methods (two self rating seales, and one psychiatric observation rating) was not more common in a sample of heterogenous chronic pain patients than in normal controls. Moreover, the three measures employed were found to be uncorrelated, and the authors question the validity of the construct.

What can be concluded concerning discordance and chronic pain? The best confirmed relationship seems to be between chronic pain and behavioural-cognitive discordance. However, there appears to be no etiological role for this form of discordance. The originall model by Gannon and Haynes assumes cognitive-physiological discordance to be of etiological significance in chronic pain (among other disorders), however this relationship turns out to be hard to verify as the physiological correlates of most types of chronic pain are largely unknown. Laboratory studies of experimental pain could be useful here. The physiological correlates which accompany experimental pain are better known, and discordance between these variables and cognitive pain variables can be assessed in the laboratory in chronic pain patients as well as in control subjects. This should make clear whether chronic pain patients do in fact display such discordance behaviour.

Finally, indications have been found that alexithymia, and the resulting discordance between physiological and emotional stress variables can have a role in the etiology of chronic pain. Again, laboratory studies could form a useful addition to clinical studies with chronic pain patients.

\subsection{Methodological considerations of psychophysiological studies}

Physiological and psychophysiological studies encounter a number of problems, which can limit their reliability and validity. The first problem, which has already been discussed above, is the selection of the appropriate target variable. The best strategy to deal with this problem is perhaps to include more than one target variable. For instance, in muscle tension studies in headache patients, not only frontalis EMG, but also trapezius, temporalis and cervical muscles are good candidates for assessment. For research in migraine patients, the selection of appropriate target variables is probably even more problematic. Besides a number of vascular variables to choose from, several muscular variables could be measured. The disadvantage of a multipleassessment strategy is of course the increasing chance of funding incidental results. Moreover, such a strategy will be time and effort consuming, and perhaps even burdensome for the subjects in the study. Thus, which and how many variables to include in an experiment should always be considered carefully, based on the weighing of probable costs and expected effects of the inclusion of an extra variable.

Another problem in psychophysiological studies is the procedure of data acquisition and analysis. For instance, with EMG measurement, many difficulties have been described which can complicate the interpretation of results. Absolute levels of tension are difficult to compare between subjects, because they can be influenced by factors such as the position of the electrode in relation to the muscles, minimal differences in body position, thickness of the skin overlying the muscle, etc. 
(Biedermann, 1984; Biedermann, 1986). The parameters of EMG data analysis also deserve consideration. Filtering characteristics have differed widely between studies and many different sampling and scoring procedures have been used. Measuring vascular variables does not pose less methodological problems. For instance, when measuring digital pulse volume, artifacts as a result of movement or temperature can easily occur. For some wariables, no consensus exists on how they should be quantified.

Another problem arises because often the comparability between studies is hindered by differences in experimental manipulations or the conditions under which measurements are made. In studies of paraspinal muscle activity in back pain patients for instance, the position in which the patient was assessed proved to be important. Therefore, one can not compare the results of studies which measured muscle activity in sitting patients with those that measured muscle activity in standing patients. Another source of variability in psychophysiological experiments might be the inchusion of an adaptation or relaxation period, to allow for the physiological parameters to stabilize. Such a period is not always present, or varies in length between studies. In stress studies, a major source of variance is the nature of the stressor. Stressors cannot be lumped together without distinction. A mental arithmetic test will not have the same effect on a subject as discussing stressful events, although both are stressors. The need for relevant stress procedures has been emphasized before.

\subsection{Conclusion}

It is not easy to formulate a general conclusion regarding the significance of psychophysiological etiological models for chronic pain. Results have been inconsistent, and as outlined above, methodological differences between studies often reduce the comparability. Neverthelless a preliminary conclusion concerning the various models and their relevance to chronic pain will be presented here.

The reflex-spasm model does not seem to offer sufficient explanation for the various chronic pain syndromes. The stress-reaction model on the other hand, appears to be more relevant. Both in headache research and in studies focussing on back pain, confirmation for this model has been found. For CLBP, especially the elaborated version of the stress reaction model, the diathesis stress model, is attractive. It has proven its value in at least one study, but replication of this study is desirable. The advantage of the diathesis stress model is that it makes very straightorward predictions. CLBP pateitns show an exaggerated stress response only in the paraspinal muscles, and only when the stressor is personally relevant (how to establish if a stressor was actually relevant for an individual might pose a problem). Thus, the reflex-spasm and the diathesis-stress model deserve further attention. Future studies should, however, take great care to use stressors that are actually relevant. Still better, the relevant physiological parameters could be assessed in real life stress situations. New technical developments of ambulatory recording equipment could make this approach possible. 
The second model which might be of value is the biomechanical model. This model pertains solely to CLBP. Aspects of the biomechanical model have received support from several studies. However, whether the aberrant patterms of muscular activity which are found in back pain patients are caused by postural abnomalities formed during the acute pain phase, and whether they ultimately cause pain by destabilizing the spine and inducing mechanical irritation, has not been ditectly tested. Therefore, only partial confirmation is available. Still, the model certainly deserves further study.

The cognitive-plnysiological discordance and the alexithymia model seem interesting, but testing of this model has hardly been done yet. Probably, laboratory research is preferable over correlational studies between (perhaps irrelevant) physiological and cognitive variables at this stage.

\subsection{Questions addressed in this dissertation}

The studies in this dissertation specifically deal with CLBP patients. The diathesisstress model, the biomechanical model and the cognitive-physiological discordance model are considered to be most relevant for CLBP, and therefore, these are the models which have been addressed by several of the studies presented in this thesis.

It was stated above that the Flor et al. study and the Arntz et al. study of the diathesis stress-model deserve replication. In section 5.2 the hypothesis that CLBP patients react to an aversive stressful stimulus with more pronounced paraspinal muscle responses is tested. A tonic pressure pain stimulus was chosen as the stressor. This pain stimulus has been described as more clinically relevant than most other short, phasic pain stimuli. It gives a gradually increasing dull pain, which is usually experienced as rather aversive. This stressor was considered to be relevant for chronic pain patients. The diathesis-stress model assumes that after the pain complaint has been established, pain itself can serve as a new stressor. The model also predicts that only specific responses, i.e. in back muscles, are increased. Therefore, paraspinal EMG was assessed. To test for specificity, also other, non-specific, physiological wariables were measured; frontal muscle $\mathrm{EMG}$, skin conductance, heart rate and respiration. On the basis of the literature, baseline differences on any of the muscular or autonomic variables are not expected, and the hypothesis that there are no sustained physiological differences between CLBP patients and controls is also tested.

When planning the experiment which is reported in 5.2 , there was no initial intention to test the predictions made by the biomechanical model. Yet it happened that the results of the study seemed to be most in line with this model. Therefore, in the discussion of this study, the aberrant muscular functioning in CLBP patients is briefly discussed in relation to the biomechanical model. However, as outlined in section 2.4 the biomechanical model should now be tested in a longitudinal study. Such a study was beyond the scope of this dissertation.

In the previous section, a case was made for a laboratory model of discordance, in which subjective and physiological responses to an experimental pain stimulus could 
be correlated. This has been done in the studies reported in sections 5.4 and 5.9 . Section 5.4 reports on correlational data from two separate studies not specifically aiming to measure discordance at the outset, but in which both cognitive (subjective) and physiological parameters were assessed, allowing for testing of discordance. In the first part of this section it is tested whether there is a relationship within subjects between the subjective painfulness scores and magnitude of skin conductance responses. If CLBP patients have a discordant response pattern, low correlations between the subjective painfulness of a stimulus and the magnitude of the SCR to this stimulus will be found. Concordance in control subjects will be shown by higher correlations between these variables. In the second part of section 5.4 , parallel changes in subjective and physiological responses are investigated. Usually, after repeating a pain stimulus of constant intensity, subjective and physiological responses decrease in intensity (see also the section on habituation). It is investigated whether the decreases in the cognitive and physiological system are synchronous or desynchronous in CLBP patients and controls.

Finally, in section 5.9 the subjective pain threshold is correlated with the threshold of a nociceptive muscle reflex (see for the discussion of this reflex section 3.6). It has been shown in previous studies that in normal subjects these two variables are closely related (concordant). Whether the same relationship holds for CLBP patients is investigated in this last study. It will also be established whether the reflex threshold and the pain threshold change synchronously. 


\section{CHRONIC PAIN AND EXPERIMENTAL PAIN SENSITIVITY}

\subsection{Introduction}

For some time now, laboratory studies have been carried out which assessed sensitivity for experimental pain stimuli in chronic pain patients. One of the motives for such studies was the assumption that altered pain sensitivity can be a factor contributing to the chronic pain experience. By contrast, it has also been argued that differences in experimental pain perception of chronic pain patients can be viewed as a consequence of their longstanding experience with pain. The latter view has become predominant in recent years.

This chapter will begin by reviewing five theoretical interpretations of why chronic pain patients would have a different pain sensitivity. The two interpretations to be discussed first argue that chronic pain patients are hypersensitive to pain signals, while the next three interpretations assume chronic pain patients to be hyposensitive to pain signals. Whether chronic pain patients are hyper- or hyposensitive to experimental pain, can be tested in the laboratory. Several sudies have compared the responses of chronic pain patients and pain-free controls to experimental pain stimuli. An overview of these studies will be presented, and a preliminary conclusion as to experimental pain perception in chronic pain patients will be drawn from these studies. Finally, a new model of altered pain sensitivity in chronic pain patients is proposed, based upon a dual mechanism, which can better accommodate the various findings than previous single mechanism models."

\subsection{Hypochondriasis}

Increased sensitivity for all kinds of internal signals, including pain, could result from the existence of hypochondriacal tendencies in chronic pain patients. It has been suggested that hypochondriasis can play a role in the development and maintenance of chronic pain. The DSM-III-R (American Psychiatric Association, 1987) defines hypochondriasis as a preoccupation with the fear of having a serious disease, which is based on the interpretation of physical signs or sensations as evidence of physical illness. In addition, there is no physical disorder that can account for these signs.

Although there is some general consensus about what hypochondriasis globally

\footnotetext{
'Throughout this chapter. "altered pain sensitivity" will be used to refer to any change in pain threshold, pain tolerance or any other pain variable, indiscriminate of whether this is due to changed nociception, a change in perceptual sensitivity, or a change in the willingness to report pain.
} 
signifies, the term has been given different emphasis. In 1983, Barsky and Klerman gave four different conceptualizations of hypochondriasis (Barsky \& Klerman, 1983). The conceptualization most relevant for the present discussion is that of hypochondriasis as a perceptual and cognitive deficit. Two components can be distinguished in this conceptualization. First, normal bodily sensations are amplified and augmented. This means that a hypochondriacal patient experiences these sensations as more noxious and more intense than non-hypochondriacal individuals. Attention and anxiety play a prominent role in the amplification of somatic symptoms. Chapman has previously described a similar phenomenon under the name hypervigilance: a perceptual habit of vigilance for somatic distress signals (Chapman, 1978). According to Chapman $_{\text {this }}$ thabit is established by prior learning processes. The second component of this conceptualization of hypochondriasis is the occurrence of cognitive misinterpretation of internal sensations. Trivial symptoms are mistaken for the symptoms of a serious disease. The resulting anxiety further exacerbates these symptoms. In sum, hypochondriacal individuals meticulously monitor all sensations felt within the body, and are alarmed by them. Internal sensations are readily attributed to a disease process, rather than to an insignificant and transitory physical state.

Tyrer et al. (1980) has tested the hypothesis that hypochondriasis is characterized by increased perceptual sensitivity. Patients diagnosed as hypochondriacal were shown to have a greater awareness of bodily sensations than non-hypochondriacs: they could estimate their heart rate more accurately ${ }^{2}$. Scores on the Whiteley Index of Hypochondriasis (a cognitively oriented questionnaire) have been found to correlate highly with the scores on a questionnaire measuring the self-reported sensitivity to all kinds of neutral and unpleasant sensations and stimuli (Barsky \& Wyshak, 1989). Pain sensitivity was shown to be increased by hypochondriasis in a study of Bond (1971). He demonstrated that women with cervix carcinoma who experienced pain, scored higher on the Whiteley Index of Hypochondriasis than women who did not experience pain. Also, a study of Bianchi (1971) has shown that individuals showing disease phobia (closely related to hypochondriasis) could detect the presence of an electrical stimulus at a lower voltage, and were less pain tolerant for this stimulus than control subjects.

The proposed mechanisms of increased perception of bodily signals, attention and anxiety, have been tested in several studies. Some studies have experimentally manipulated attention to internal versus external signals. Pennebaker demonstrated that when subjects exercising on a treadmill were prompted to process internal sensory information by presenting them with feedback of their own respiration, a larger number of symptoms (like sweating and headache) and more fatigue were reported than when subjects heard distracting sounds (Pennebaker \& Lightner, 1980) or when they could freely attend to external cues (Pennebaker, 1982). Oosterlaan \& Schmidt (unpublished data) have shown that merely instructing normal healthy subjects to pay attention to a specific part of the body caused them to report a substantial number of

2 However, previous research has shown that a greater accuracy in perceiving internal signals does not always comcide with increased symptom reporting (Pennebaker, 1982). 
sensations in that part of the body on a checklist presented afterwards. Reversely, in other studies it has been shown that when more extemal cues are available, a subject will distract attention from internal cues and reports fewer physical symptoms. Pennebaker (1982) reports several experiments demonstrating this so called cue competition. Ahles et al. (1987) showed that relatively stable individual differences. exist in the processing of internal information. Subjects who were predisposed to attend to somatic symptoms, as measured by the body consciousness scale, and subjects who were anxious, reported more areas of spontaneous pain when invited to do so. In addition, they rated this pain as more intense than the control group.

The influence of anxiety on the perception of experimental pain stimuli has been tested in several studies. Often, an increase of pain sensitivity was found during conditions which provoke anxiety (Haslam, 1966; Hill et al., 1952; Cornwell \& Donderi, 1988). However, a recent study by Arntz et al. (1991b) showed that the effect of anxiety on pain perception may be mediated by an attention effect. Anxiety directed at a pain stimulus, increasing attention to this stimulus, did enhance pain sensitivity, but anxiety directed away from a pain stimulus, towards an external event and thus distracting attention away from the pain, decreased pain sensitivity.

The next question is whether there is a link between hypochondriasis and chronic pain. Hypochondriasis has been implicated as a risk factor in the development or maintenance of chronic pain. Because hypochondriacal individuals have an increased sensitivity for all kinds of somatic signals, they will also perceive pain signals sooner. Once pain is felt, continuing attention to it, in combination with a misinterpretation of the pain as a symptom of a serious disease, will intensify this pain still more. Increased screening of pain sensations can also be secondary to an acute pain episode. A conceivable sequence of events in this case would be that after an initial pain occurrence, an individual learns to focus on the internal signals, to detect whether "the pain is still there". This attention to pain signals can subsequently develop into a habitual behaviour pattern, causing pain to persist. Finally, hypochondriasis could be secondary to chronic pain, caused by the anxiety which often accompanies pain states.

Several studies have tested whether chronic pain patients are in fact characterized by increased hypochondriacal tendencies. Some confirmation thas been found for this. Well-known is the MMPI profile often found in chronic pain patients, with elevated scores on the hysteria and hypochondriasis scales, and a smaller elevation of the score on the depression scale, the so-called conversion- $V$, or with elevated scores on all three scale, the neurotic triad (Sternbach et al. , 1973; Sternbach, 1974; Calsyn et al., 1976; Freeman et al., 1976; Cox et all., 1978; Naliboff et al., 1985). Watson (1982) compared the scores of chronic pain patients on the MMPI with those of a large number of other medical patients not complaining of pain. Only in the pain group, a significant subset of patients was found, who complained of vague and diffuse somatic symptoms, characteristic of the hypochondriac. Pilowski has used the concept of "Abnormal Illness behaviour" to indicate a behaviour pattern characterized by (1) general hypochondriasis, (2) disease conviction, (3) a somatic view of illness, (4) affective inhibition, (5) denial of life problems and (6) irritability. These factors can be assessed by means of the Illness Behavior Questionnaire (IBQ), developed by 
Pilowski \& Spence (1975). A study with chronic pain patients has found that they showed a greater disease conviction, somatic focussing and denial of life problem in comparison to general practice patients.

Another kind of evidence that chronic pain patients may be hypervigilant to somatic signals comes from the studies by Waddell et al. (1980) and Korbon et al. (1987). Both have found that CLBP patients often showed signs of hypersensitivity during pseudo-physical examination. Procedures not normally painful, elicited pain responses in subjects in the patient group. Waddell also found a (moderate) correlation with these so called non-organic signs, and the score on the MMPI hypochondriasis scale. Another study, by Schmidt et al. (1989), demonstrated that CLBP patients reported a great number of spontaneous interoceptive sensations during rest conditions, whereas a control group of healthy subjects reported almost no such sensations during rest, but only after physically exercising to tolerance.

In conclusion, there are some indications that chronic pain patients are indeed more hypochondriacal. Hypochondriasis is characterized by increased awareness of somatic signals, including pain. This implies that if hypochondriasis is a feature of chronic pain patients, they will show increased sensitivity for experimental pain stimuli in comparison to pain-free controls. With laboratory tests, a greater sensitivity to both experimental pain and other somatic signals is predicted in chronic pain patients.

However, a word of caution on the use of terminology and measuring instruments is indicated here. As stressed above, hypochondriasis is not unambiguously conceptualized. Hence, the studies referred to have used different definitions of hypochondriasis, and different measuring instruments. For instance, the Whiteley Index operationalizes hypochondriasis as a bodily preoccupation, a disease phobia which the patient seeks to deny, and a disease conviction. Thus, it is a cognitively oriented questionnaire. The hypochondriasis scale of the MMPI on the other hand, assesses the presence of all kinds of non-specific sensations and somatic complaints. Cognitive aspects of hypochondriasis are ignored in this questionnaire. Therefore, the meaning of hypochondriasis is difficult to compare between the various studies. In addition, it has been suggested that the elevation on the hypochondriasis scale of the MMPI in chronic pain patients might be due to the items it contains. These items, asking for the presence of physical sensations, would possibly simply reflect the actual poorer health of chronic pain patients. Therefore, Watson (1982) performed an analysis on the basis of individual items of the hypochondriasis scale. With this procedure there were still indications of hypochondriacal tendencies in chronic pain patients.

\subsection{Learning processes and stimulus generalization}

Increased pain sensitivity in chronic pain patients could also be the result of generalization of behaviour patterns previously established by operant learning. Operant learning processes have been implicated in the development and maintenance of chronic pain. In the operant pain model, the emphasis has been on overt and observable expressions of pain. Reinforcement of pain behaviours (and sometimes concomitant punishment of well behaviour), causes these behaviours to persist, 
increasingly disabling a patient. Pain behaviours can be reinforced in two different ways (Fordyce, 1983). First, the expression of pain can elicit positive consequences from the environment (e.g. the attraction of sympathy and attention). This positive reinforcement will probably mainly establish such behaviours as grimacing and complaining of pain. Second, some forms of pain behaviour can initially lead to a reduction of nociceptive stimulation in the acute pain phase, and thereby avoid negative consequences (pain). This negative reinforcement probably mainly establishes such behaviours as guarding and limping.

Avoidance learning is one of the most prominent learning processes of chronic pain patients. Activities that once led to an increase of pain are subsequently avoided. Once avoidance behaviour is established it may be extremely resistant to extinction (Fordyce, 1983). Avoidance is self-sustaining: the thought that by refraining from an activity pain has been averted is in itself a reinforcer. Moreover, the persistence of avoidance prevents the occurrence of extinction because a subject never gets the chance to experience that the avoided activity does not cause pain anymore. Cognitive consequences of avoidance can also serve to maintain it. The avoidance of stimulation results in a reduction of the feeling of control over pain, and it increases the expectation that exposure will increase pain. Such cognitive changes further encourage avoidance and decrease tolerance of stimulation (Philips, 1987). Escape and avoidance from potentially pain eliciting situations by headache patients has been found in a study of Philips \& Hunter (1982). They compared chronic headache sufferers and controls on their assessment of and escape from strong auditory stimuli and found that headache sufferers tolerated a much narrower range of sound stimuli. When they experienced pain during the test, escape from the stimulus was especially prominent.

By means of the process of stimulus generalization, complaining about pain and avoidance of stimulation can become generalized from the chronic pain situation to the acute pain situation. According to the principle of stimulus generalization, a particular response which is at first linked to a specific stimulus, can after some time generalize to other stimuli which resemble the original one. When generalization to the experimental pain situation occurs, complaining about experimental pain stimuli will increase. In addition, generalization of avoidance behaviour will result in the avoidance and escape from experimental pain situations, and thereby to lower tolerance for experimental pain stimuli. Schmidt, in a series of experiments, has tested the scope of stimulus generalization. He found that CLBP patients not only displayed poorer persistence in a treadmill test, a task potentially eliciting pain in the back (Schmidt, 1985), but also in an acute pain situation (cold pressor test) (Schmidt \& Brands, 1986). However, in a non-painful reaction time test there were no differences between CLBP patients and pain-free controls regarding persistence behaviour (Schmidt, 1986).

In conclusion, generalization of learned behaviour patterns to an experimental pain situation can result in increased avoidance of pain stimuli, and more complaining of pain. According to this principle, experimental pain sensitivity will therefore be increased in chronic pain patients. This will be especially apparent on measures of pain tolerance. 


\subsection{Adaptation level theory}

Whereas the previous two models argue that chronic pain patients are more sensitive to experimental pain, a decreased pain sensitivity can be hypothesized on the basis of the adaptation level theory. The adaptation level theory which was formulated by Helison in 1964, is a theory concerning the perception and judgement of stimuli in general. According to this theory, all judgments are based upon three factors: characteristics of the stimulus to be judged, the context in which this stimulus occurs and individual internal standards for judgement (based on experience). The adaptation level theory has been applied to the perception and judgement of pain.

Rolman (1979; 19796 ) experimentally demonstrated the occurrence of adaptation level effects on pain judgement. He described the so called zig-zag phenomenon of intensity judgement. In his experiments, four stimuli of increasing intensity (s1, s2, s3 and 84 , respectively) had to be judged by a subject. $\$ 2$ was always paired with a weaker stimulus ( $\$ 1$ ), whereas $\$ 3$ was always paired with a stronger stimulus (s4). The result of this pairing was that $s 3$ was rated as less intense than $s 2$, contrary to the actual situation. This can be explained by the fact that the reference point (or adaptation level) for $s 3$ was one of higher intensity, and therefore 33 was judged as relatively mild. When all four stimuli were presented in random order, this zig-zag effect disappeared, and intensity ratings increased linearly with stimulus intensity. Thus, the experiment showed that the intensity rating of a pain stimulus is dependent on the intensity of previous pain stimuli.

For chronic pain patients, the adaptation level theory predicts that they judge an experimental pain stimulus in relation to their clinical pain. As a result of the clinical pain, both the context in which the experimental pain stimulus occurs and the internal standard of judgement, are different for chronic pain patients than for pain-free individuals. The context is a concurrent or remembered painful state, and the adaptation level is expected to be higher because of a patient"s extensive experience with pain. As a result, experimental pain stimuli will be perceived as relatively mild. Increased pain threshold and tolerance, and decreased intensity judgments of experimental pain, will be the result. Moreover, when the intensity of the clinical pain is more severe than the intensity of the experimental pain, the effect is expected to be especially potent (Naliboff \& Cohen, 1989).

Rollman has also speculated that the zig-zag phenomenon found in normal subjects might not be present in chronic pain patients, because of the potent adaptation level which they have avallable as a result of their clinical pain experience (Rollman, 1979; Rollman, 1979b). If all intensities of experimental pain stimuli are judged with reference to such a strong internal anchor point, external references might have less impact. However, an absence of the zig-zag effect in chronic pain patients could not be demonstrated (Cohen et al., 1983). In fact, it was found that it was the chronic pain group in which a zig-zag effect occurred, whereas it was absent in the control group.

In summary, the adaptation level theory predicts chronic pain patients to be less sensitive for experimental pain stimuli. This lesser sensitivity is the consequence of their persistent pain, which influences the context in which the judgments of additio- 
nal pain stimuli are made, and which sets a higher adaptation level in chronic pain patients.

\subsection{Activity of endogenous opioids}

Another reason for chronic pain patients to become less sensitive to acute pain could be pharmacologically based. Some twenty years ago, it was demonstrated that the body itself produces morphine-like peptides. These peptides, the so-called endogenous opioids, bind to specific opioid receptors within the central nervous system, the same receptors to which morphine and other pharmaceutically produced opiates bind. Moreover, endogenous opioids have been found to posses analgesic properties. In the course of time, various types of naturally occurring opioid peptides have been identified: $\beta$-endorphin, enkephalins (with two subtypes, met- and leu-enkephalin) and dynorphin. In addition, different opioid receptor types can be distinguished, with $\mu-, \delta$ and $k$-receptors being the most important. The role played by $\mu$-receptors in analgesia is unchallenged, the analgesic effect of morphine for instance, is established by its binding to the $\mu$-receptor. In addition, recent research has demonstrated that probably $\delta$ - and $\kappa$-receptors are involved in analgesia as well (Millan, 1986). The affinity of the various endogenous (and exogenous) opioids for the receptor types differs. $\beta$-Endorphins are shown to have the greatest affinity for $\mu$ and $\delta$-receptors, met-and leuenkephalin for $\delta$-receptors and dynorphin for $\kappa$-receptors (Teremius, 1987).

Endorphin, dymorphin and enkephalins are not equally distributed within the CNS. $\beta$-Endorphin is only found in the brain, predominantly in the pituitary and hypothalamus. The enkephalins, especially met-enkephalin, are more broadly distributed. They are found not only in the brain, but also in the spinal cord, mainly in interneurons of the substantia gelatinosa, an area which has often been implicated in pain control. In addition, enkephalins have also been found in the peripheral autonomic nervous system. Dynorphin is also found both in brain areas (periaquaductal grey, limbic system, thalamus) and within the dorsal horn of the spinal cord. Its distribution within the cord is somewhat more restricted than that of the enkephalins.

Research into the physiological significance of the endogenous opioid system and the natural ways of activating this system has advanced owing to the availability of opioid antagonists. An opioid antagonist is a substance which binds to the opioid receptor site, without activating it. Thereby, the receptor is blocked for the effective agonistic substance. One of the most extensively used opioid antagonist is naloxone, a preferential antagonist for the $\mu$-receptor, but with effects on the other receptor types as well (the affinity for $\mu$-receptors is about 10 times as potent as for the other receptor types; Goodman, 1985). Naloxone has been used to study the degree of spontaneous activity of the endogenous opioid system. If there is tonic activity of this system, administration of naloxone would lead to hypersensitivity for pain stimuli in normal subjects. In several animal studies, hyperalgesia after nalloxone administration has been found (for refs, see Mayer, 1979), although there are indications that actually the effecti is bi-directional, with low doses of naloxone leading to hypalgesia and high doses leading to hyperalgesia (Woolf, 1980; Dïckenson et al ", 1981b; Ueda 
et al., 1986; Jurna, 1988). Sudies with healthy human subjects have usually not been able to find an effect of naloxone on subjective pain perception or on nociceptive reflexes (El-Sobky et al, 1976; Grevert \& Goldstein, 1978; Goldstein, 1979; Terenius, 1982; Stacher et al., 1988; however: Buchsbaum et al. 1977). There might be some exceptions, however. Patients in whom a congenital insensitivity to pain had been established, and who normally had very high nociceptive thresholds, demonstrated a lowering of these thresholds to nomal values after administration of naloxone (Willer et al., 1978; Dehen et al., 1978). This suggested that congenital insensitivity to pain is related to a toric release of opioid peptides.

Although normally ongoing release of endogenous opioids in human subjects does not seem to occur, several conditions can stimulate such release. One of these conditions is pain. Acute pain stimulation was found to be associated with increased release of met-enkephalin-like material (Hamon et al, 1988). It has been suggested that chronic pain could similarly give rise to an increased production and release of opioids, thereby rendering the patient less sensitive to pain. This hypothesis has been tested by means of two different methods. The first method is to administer naloxone to chronic pain patients, and to test whether clinical pain is exacerbated, or experimental pain sensitivity increased. The second method directly measures opioid peptide levels in the cerebrospinal fluid (CSF) or blood plasma of chronic pain patients.

Studies administering naloxone to chronic pain patients have failed to show an effect on pain sensitivity. Lindblom \& Tegner (1979) found no alteration of spontaneous (clinical) pain, nor of experimental heat pain threshold, in ten patients with neuralgia or low back pain after administration of naloxone. Langemark (1989) neither found an aggravation of clinical pain in chronic tension headache patients with naloxone. However, studies with acute post-operative pain have shown that the effect of naloxone might be dose dependent. Levine et al. (1978) administered $9 \mathrm{mg}$ Naloxone i.v. to patients experiencing pain resulting from the removal of an impacted mandibular third molar, and found that the pain increased. Skjelbred \& Lokken (1983) found no effect with patients who had been undergoing the same surgical procedure upon administering a lower dose, namely $4 \mathrm{mg}$. The bi-directional effect of naloxone was directly demonstrated by Levine et al. (1979): low doses $(0.4$ and 2 $\mathrm{mg}$ ) of naloxone produced analgesia, while high doses $(7.5$ and $10 \mathrm{mg}$ ) produced hyperalgesia in post-operative dental surgery patients. Therefore, the negative results found in the studies with chronic pain patients are possibly due to the administration of a dose of naloxone too low to induce hyperalgesic effects. Langemark used $4 \mathrm{mg}$, and Lindblom and Tegner used only $0.8 \mathrm{mg}$ i.v. Thus, on the basis of naloxone studies, no conclusion as to whether chronic pain patients have a higher endogenous opioid activity can be drawn yet.

The second method, direct measurement of opioid peptides in the cerebrospinal fluid (CSF) or blood plasma of chronic pain patients, has neither produced unambiguous results. Indications that opioid activity is enhanced by chronic pain comes from the study of Alkinson et al. (1983). They found chronic pain patients (with physical findings insufficient to explain the degree of invalidity) to have a significantly higher level of $\beta$-endorphin in the blood plasma compared to psychiatric patients and healthy volunteers. However, instead of higher opjoid levels in the CSF or blood plasma of 
chronic pain patients, lower lewels have been reported more frequently. Denko et al. (1982) found a lower plasma level of $\beta$-endorphin in patients with rheumatic disorders, and lower CSF levels of $\beta$-endorphin were found in migrame patients (Genaz zani, et al. 1984; Nappi, et al., 1985). Another study found decreased metwenkephalin levels in the CSF of cluster headache patients, although the $\beta$-endorphin level was normal (Hardebo, et $\mathrm{al}_{\text {, }}$ 1985). Terenius (1987) suggested that the nature of the chronic pain might determine whether increased or decreased opioid levels are found. Almay et al. (1978) found that whereas CSF endorphin levels in patients with organic pain syndromes were decreased, especially in those patients with clearly positive neurological signs of peripheral nerve lesions, "psychogenic" pain patients showed a tendency towards increased endorphin levels. This finding was replicated by Terenius (1987), who found decreased opioid peptide CSF levels in neurogenic pain patients, and a somewhat higher level in idiopathic pain patients.

All of these studies have implicitly assumed that plasma and CSF opioid content are valid indicators of opioid activity. However, this premise might be questioned. Especially in the case of plasma opioid peptide level the functional significance is not clear. Enkephalins are very rapidly broken down in the blood and therefore do not likely have a major analgesic function. But the analgesic role of plasma $\beta$-endorphin levels can also be questioned, because $\beta$-endorphin can hardly cross the blood brain barrier. Moreover, it was found that CSF and plasma $\beta$-endorphin levells do not correlate (Terenius, 1979). Directly contradicting an analgesic role of $\beta$-endorphin plasma levels is the study of Genazzani et al. (1986), which did not find a correlation between (experimentally increased) Jevels of plasma $\beta$-endorphin and pain threshold, pain tolerance and a nociceptive reflex in healthy volunteers. The functional significance of CSF levels of opioid peptides is perhaps more straightforwand. However, the origin of peptides in the CSF is not clear. Opioids and opioid metabolites found in the CSF can be of both spinal and supraspinal origin (Facchinetti et al., 1988). In addition, the mechanism responsible for a changed opioid level does not become clear by just assessing CSF content. It might reflect alterations in either synthesis, release or degradation of opioids. Finally, the opioid content found depends on the particular measure which is used to assess opioid peptides in the CSF. Two different methods are available, one based upon the measurement of receptor active material (receptor assay), and one based upon measurement of compounds with a specific structure (radio-immuno assay) (Terenius, 1979). These two methods produce markedly different absolute levels of total opioid content (Terenius, 1988).

Animal studies can perhaps better elucidate how pain influences the endogenous opioid system. The model which is available for studying chronic pain in animals is that of induced polyarthritis in the rat. Rats are infected with mycobacterium butyricum, which induces multiple sites of inflammation, lasting for several weeks and reaching peak severity in about 3 weeks. In the active phase of the disease, these rats show a rise in experimental pain threshold, suggesting that endogenous opioids might be active (Calvino \& Le Bars, 1986; Millan et al., 1987; Millan et al., 1988). The main result from studies with arthritic rats has been the finding that in particular the synthesis of opioid peptides is increased. B-Endorphin synthesis was found to be enthanced in the anterior pituitary (Millan et al., 1986; Millan et al., 1987). However, 
most research in animals has concentrated on the synthesis of dynorphin. This has also been found to be increased in chronic arthritic rats, both in the anterior pituitary and halamus (Millan et al. 1986). In addition, the content of dynorphin in spinal cord tissue was enhanced, and this seemed to be due to facilitation of the biosynthetic activity of the dynorphin neurons within the spinal cord (Millan et al., 1987; Millan et al., 1988; ladorala et al., 1988). The level of enkephalin within the spinal cord was similarly found to be increased in rats with arthritis, although to a lesser extent than dynorphin (Millan et al., 1986) and only during the worst stage of the disease (Millan, er al., 1988).

In conclusion, chronic pain seems to increase the synthesis of opioid peptides. However, animal studies have also shown that enhanced synthesis of these peptides is not necessarily reflected in the CSF opioid level. Both for met-enkephalin-like material and dynorphin it was found that an increase in spinal cord tissue can coincide with a diminished release into the CSF (Przewlocka et al., 1986; Bourgoin, et al, 1988; Kayser, et al., 1988). The value of CSF opioid level assessments in human chronic pain patients can therefore be questioned. This is illustrated by the finding that patients with congenital insensitivity to pain (who did react to the administration of naloxone) had normal CSF levels of met-enkephalin-like material (Cesselin et al., 1984) and $\beta$-endorphin (Dehen et al., 1986). Dynorphin levels were not assessed, like in most other human studies, although animal research has indicated that this opioid is equally important, if not more important as a correlate of chronic pain (at least in animals). Finally, when synthesis of opioids is increased this does not necessarily lead to analgesia. There is a serious possibility that tolerance to endogenous opioids will develop. With morphine, tolerance comes about rather rapidly. Similarly, tolerance to $\beta$-endorphin and met-enkephalin has been found (Terenius, 1979). Therefore, even if the synthesis of endogenous opioids is enhanced by chronic pain, it remains to be determined whether this will lead to decreased pain sensitivity.

\subsection{Counterirritation and DNIC}

The most recent hypothesis of how chronic pain can influence acute pain perception has been advanced by Le Bars \& Willer (1988). They have suggested that counterifritation and its proposed neurophysiological basis DNIC could be the mechanisms responsible for a decreased pain sensitivity in chronic pain partents. In this section, the concept of counteritritation will be explained first, and the parallels with diffuse noxiows inhibitory controls (DNIC) will be emphasized. Next, the evidence that counterirritation and DNIC can play a role in chronic pain is reviewed.

Counterirritation is the phenomenon that one pain leads to the inhibition of another pain. Counterirritation has also been described under the names of counterstimulation and hyperstimulation analgesia. Clinically, the effect has been known and applied for centuries. A number of historical applications of counteriritation in the treatment of rheumatic and arthritic pain have been described by Wand-Tetley (1956). Most of then seem rather cruel nowadays, they included methods like scarification of the skin, burning the skin with a hot iron and making blisters and open sores. Later 
applications based upon the same principle can be found in the use of electricity for pain relief, which was popular in the 19 th century. Also, some techniques in current pain treatment practice have been ascribed to the operation of counterirritation. One of these techniques is acupuncture analgesia, which according to some is based on the induction of a (near) painful experience, the "teh ch " $i$ " sensation (Le Bars et al, $1979 \mathrm{~b}$ ). Another technique for pain relief which could be based upon counterirritation is transcutaneous electrical nerve stimulation (TENS), at least the high-intensity, low frequency form of TENS (Willis, 1982).

Several studies have experimentally established the analgesic effect of counterirritation. Le Bars et al. (1989) present a number of studies from the first half of this century which already documented the influence of one experimental pain upon the other. More recent studies have also been performed. Usually these have assessed the influence of a relatively long and diffuse pain stimulus on the perception of a more localized and short pain stimulus (Anderson et al., 1976; Pertowara et al., 1982; Chen \& Treede, 1985; Talbot et al., 1987; Pantaleo et al., 1988; Price \& McHaffie, 1988). One study demonstrated the opposite effect: a short phasic pain was able to inhibit the perception of more tonic pain resulting from the cold pressor test (Riley \& Levine, 1988). Studies using physiological correlates of pain to study counterirritation have also been performed. Chen et al. (1985) demonstrated that a tonic pain stimulus reduced the amplitude of the cerebral evoked potential to a phasic pain stimulus, in addition to the reduction of the subjective pain rating. Pantaleo et al. (1988) measured the subjective pain threshold and the blink reflex associated with an electrical pain stimulus and found both to be decreased during a simultaneous ischemic pain stimulus.

A powerful design to test for antimociceptive effects was found in the measurement of the nociceptive flexion reflex of the biceps femoris. The elicitation of this reflex was described by Hugon (1973). Stimulating the nervus suralis by means of surface electrodes within the receptive field of this nerve can elicit a polysynaptic nociceptive reflex of the short branch of the biceps femoris. This reflex can be measured with surface electrodes in the bend of the knee, above the appropriate muscle. With low stimulus intensities, initially only a short latency tactile reflex is seen. When stimulus intensity increases to a level high enough to activate pain fibers, a second, nociceptive, reflex is elicited. Because pain fibers have a smaller diameter and a lower conduction velocity, this second reftex has a longer latency (75-150 msec). The conduction velocity, calculated from the latency time, shows that group III afferents (As fibers) are involved, and therefore the reflex is called the RIII reflex.

Willer (1977) has shown that there is a close correspondence between the RIII reflex and subjective pain sensation. The reflex threshold and pain perception threshold for the electrical stimulus are quite similar, and the amplitude of the reflex and the pain intensity ratings increase in a largely parallel fashion with increasing stimulus intensity. Hence, the RIII reflex is considered to be a good objective indicator of nociceptive activity, and it is well suited to measure nociceptive inhibition. A great deal of work on the effect of counterirritation on the RIII reflex has been performed by Willer and colleagues. They have sthown that several experimental pain stimuli can increase the reflex threshold, in parallel with an increased pain 
Often, the terms counterirritation and DNIC are used interchangeably. However, the phenomera referred to are not the same. Counterimitation denotes a process which decreases pain experience, and therefore can only be established in conscious human or animal subjects ${ }^{3}$. DNIC on the other hand is an anti-nociceptive mechanism which can be demonstrated in anaesthetized animals. It decreases spinal nocicepfive activity, not necessarily accompanied by a decreased pain experience in the conscious animal. However, the assumption that DNIC constitutes the neurophysiological basis of counterimitation seems very plausible. This assumption is based upon a number of similarities between the two mechanisms.

Both DNIC and counterirritation are only activated by clearly nociceptive stimuli. In animal research it was found that the degree of inhibition of convergent neurons was directly related to the strength of the eliciting stimulus (Le Bars et al., 1989). The same relationship has been found between the intensity of a counterirritation stimulus and the degree of inhibition of the RIII reflex and subjective pain perception (Le Bars et al., 1989, Willer et al., 1989). Another similarity between the two phenomena is the involvement of supraspinal structures. DNIC is abolished after transection of the spinal cord in rats. Intact supraspinal connections are also essential for the effectiveness of counterintitation in thumans. In tetraplegic patients, and in patients with a lesion of the brain stem, the RIII reflex could not be imhibited by a counterirritation stimulus (Roby-Brami et. al., 1987, De Broucker et al., 1990).

The final inhibitory effect of DNIC is exercised at a spinal level. From the inhibition of the RIII reflex, which is exclusively spinally organized, it becomes clear that counterirritation also has a spinal anti-nociceptive effect. One last similarity between DNIC and counterirritation is that both can be reduced by systemic administration of low doses of naloxone (Willer et al., 1990). Apparently, in both mechanisms endogenous opioids are involved.

What is the evidence that counterirritation and DNIC can be activated by chronic pain? In most studies the focus has been on the activation of DNIC and counterirritation by experimental pain stimuli. Yet, evidence has been found that clinical pain can have a counterirritation effect as well. Willer et al. (1985) demonstrated that patients experiencing pain from orthopedic surgery had a considerably higher RIII reflex threshold than which is normally found. In a subsequent experiment, it was demonstrated that pain from sciatica also depressed the RIII reflex (Willer et al., 1987). Performing the straight leg raising test (Lasegue's manoeuvre) on the painful side in patients wilh unilateral sciatica elicited an electrical pain radiating into the foot or the back. The same manoeuvre on the other side did not result in pain. Simulaneously with the manoeuve, the RIII reflex was recorded. The reflex amplitude decreased dramatically when the manouvre was performed on the painfull side, but not when it was performed on the non-painful side.

These studies show that clinical pain can have a counterirritation effect. However,

"Whether pain experience can be measured in animal experiments is not uncontroversial. Howeveir, animal studies are very commonly applied in pain research. Usually, reflexive or escape behaviours in response to a nociceptive stimulus are assessed, and taken as the indicators of arvimal pain experience. 
both experiments concerned acute pain. The occurrence of DNIC as a result of more chronic pain has been tested in polyarthritic rats. On a behavioural level, it was shown that these rats were less sensitive to heat pain stimuli (Colpaert, 1979; Millan et al., 1987) and visceral chemical pain stimuli (Calvino \& Le Bars, 1986). It could also be shown electrophysiologically that arthritic rats had reduced nociceptive activity in response to heat pain stimuli. The threshold of activation of dorsal horn neurons responsive to noxious heat was shown to be significantly higher in arthritic rats (Menetrey et al., 1988). In addition, in these rats DNIC could not only be activated by nociceptive stimuli, but also by gentle mechanical stimuli applied to the inflamed areas (Calvino et al., 1987; Calvino et al., 1988). Such stimuli are not normally painful but in arthritic rats they are.

Together, these studies indicate that clinical pain, acute as well as more or less chronic pain, can trigger DNIC. The suggestion that spontaneous ongoing pain in humans is also able to trigger DNIC should be tested further.

In the last five sections, theoretical models of why experimental pain sensitivity could be different in chronic pain patients have been outlined. Now it is time to turn to a more systematic review of the available experimental evidence for such differences between chronic pain patients and controls.

\subsection{Review of studies assessing sensitivity for experimental pain in chronic pain patients}

Several studies have tested whether there are differences in pain sensitivity between chronic pain patients and healthy controls. Comparing the results of these studies is not always easy because a variety of diagnostic groups has been studied, with various types of pain stimuli, and different dependent variables.

How pain sensitivity can be measured experimentally has been the topic of numerous publications (Lynn \& Perl, 1977; Procacci, 1979; Procacci et al., 1979; Wolff, 1980; Anton et al., 1984). A large number of different pain stimuli are available, all with their specific advantages and disadvantages. Procacci et al. (1979) sum up some of the basic rules with which all pain stimuli which are used have to comply. These are: (1) the stimulus needs to be measured in physical units in order to allow both a comparison between various tests and the communication of data: (2) the stimulus must induce a clearly detectable pain sensation; (3) the stimulus must not induce tissue damage or, if it does, this should be as slight as possible.

Once the choice of the particular pain stimulus to be used is made, the dependent variable has to be selected. The two variables employed most often in pain research are pain threshold and pain tolerance. Pain threshold is defined by the IASP (International Association for the Study of Pain) as the least stimulus intensity at which a subject perceives pain. Pain tolerance is defined as the greatest stimulus intensity causing pain that a subject is prepared to tolerate.

Two more parameters can be derived from the Sensory Decision Theory (SDT). 
SDT is a theory from the field of sensory psychology, and the methodology of SDT has been applied to pain research. SDT analysis yields wo different variables: discriminability and response criterion. The former is supposed to reflect al physiological process, in which a decreased ability to discriminate indicates the attenuation of physiological nociceptive activity. It would thereby be a measure of sensory sensitivity. By contrast, response criterion is supposed to reflect a psychological change, namely the willingness to report pain (bias). The premises of SDT, as applied to pain perception, have been questioned (Rollman, 1979; Rollman, 1979b; Gracely, 1979). For instance, it has been shown that an actual decrease in stimulus intensity was not reflected in a subject's discriminability score, but instead led to an apparently changed response criterion. An SDT investigator would thus have to conclude from this that the diminished pain report of a subject was due to a psychological change. Similarly, discriminability is not a valid indicator of sensory sensitivity, as it also reflects cognitive and attentional changes (Gracely, 1979). Nevertheless, stripped of its theoretical basis, pain intensity discrimination can be regarded as a useful additional variable, and therefore, this variable will be included in the present review.

Table 3.1 presents an overview of 14 studies which have assessed at least one of the variables: pain threshold, pain tolerance or pain discrimination in chronic pain patients. The most extensively studied group of chronic pain patients in relation to experimental pain perception has been that of CLBP patients. Six studies were conducted with CLBP patients, and these studies have been entered at the top of table 1 , in chronological order. The remainder of studies have focussed on a variety of pain syndromes. These are presented in the subsequent part of the table, again according to year of publication of the study. Only those studies which have used a healthy pain-free control group have been included. A second selection criterium for the studies in the table was that pain sensitivity was assessed by means of stimuli applied to sites not affected by chronic pain.

The first column indicates which study is concenned. The second column describes the patient group selected, the number of subjects in the patient and control groups and the pain stimulus which was used. The next three columns present the results of the study. Emply cells indicate that the particular variable was not measured. When no difference was found between the groups for a variable, this is indicated by " $=$ ", whereas $\uparrow$ means that a variable is higher for chronic pain patients in comparison to controls, and $\downarrow$ means that variable is lower. 


\begin{tabular}{|c|c|c|c|c|}
\hline $\begin{array}{l}\text { author(s) } \\
\text { (year) }\end{array}$ & $\begin{array}{l}\text { pain syndrome lnj/ control inl } \\
\text { pain stimulus used }\end{array}$ & $\begin{array}{l}\text { pain } \\
\text { threshold }\end{array}$ & $\begin{array}{l}\text { pain } \\
\text { tolerance }\end{array}$ & $\begin{array}{l}\text { pain } \\
\text { discrim. }\end{array}$ \\
\hline $\begin{array}{l}\text { Wolskee \& } \\
\text { Gracely }(1980)\end{array}$ & $\begin{array}{l}\text { CLBP }(n=8) / \text { (control } n=6) \\
\text { thermal pain }\end{array}$ & $\uparrow$ & & \\
\hline $\begin{array}{l}\text { Naliboff et al. } \\
\text { (19811 }\end{array}$ & $\begin{array}{l}\text { CLBP }\{n=15) / \text { controt } n=11\} \\
\text { thermal pain }\end{array}$ & $\uparrow$ & & $\downarrow$ \\
\hline $\begin{array}{l}\text { Cohen et al } \\
\text { (1983) }\end{array}$ & $\begin{array}{l}\text { CLBP }(n=11) / \text { (contral } n=11 \text { ) } \\
\text { thermal pain }\end{array}$ & $\uparrow$ & & $\downarrow$ \\
\hline $\begin{array}{l}\text { Yang et al } \\
\|1985\|\end{array}$ & $\begin{array}{l}\text { CLBP in }=551 / \text { (control } n=47 \text { ) } \\
\text { thermal pain }\end{array}$ & $\uparrow$ & & $\downarrow$ \\
\hline $\begin{array}{l}\text { Schmidt \& Brands } \\
(1986)\end{array}$ & $\begin{array}{l}\text { CLBP }(n=24) / \text { (control } n=24) \\
\text { cald pressor }\end{array}$ & & $\downarrow$ & \\
\hline $\begin{array}{l}\text { Brands \& Schmidt } \\
(1987)\end{array}$ & $\begin{array}{l}\text { CLBP (n }=21) / \text { control in }=20 \text { ) } \\
\text { cold pressor }\end{array}$ & & $\downarrow$ & \\
\hline $\begin{array}{l}\text { Hazouri \& Mueller } \\
(1950)\end{array}$ & $\begin{array}{l}\text { spinal root pain }(n=3) \\
\text { lcontrol } n=100) \\
\text { heat pain }\end{array}$ & 1 & & \\
\hline $\begin{array}{l}\text { Huskisson \& Hart } \\
(1972)\end{array}$ & $\begin{array}{l}\text { rheum, art. }(n=106) \text {, anky } \\
\text { lose spond. }(n=50) \text { (control } \\
n=50) \\
\text { pressure pain }\end{array}$ & $\begin{array}{l}\uparrow \\
\text { lonly ank. } \\
\text { spondyl.) }\end{array}$ & & \\
\hline $\begin{array}{l}\text { Molin et al. } \\
(1973)\end{array}$ & $\begin{array}{l}\text { mandibular }(n=27 / \text { / control } \\
n=26) / \text { electrical pain }\end{array}$ & $\Downarrow$ & $\downarrow$ ns & \\
\hline $\begin{array}{l}\text { Merskey \& Evans } \\
(1975)\end{array}$ & $\begin{array}{l}\text { various organ, etiol. }(n=18) \text { / } \\
\text { (control } n=10) / \text { pressure pain }\end{array}$ & $\Uparrow$ & & \\
\hline $\begin{array}{l}\text { Malow et al. } \\
(1980)\end{array}$ & $\begin{array}{l}\text { myofasc. pain } n=20) / \text { lcontral } \\
n=20 / \text { pressure pain }\end{array}$ & $\downarrow$ & & $\downarrow$ \\
\hline $\begin{array}{l}\text { Sicudds et al } \\
(1987)\end{array}$ & $\begin{array}{l}\text { fibrositis }(n=20 \mathrm{~h}, \\
\text { rheumatoid arthritis }(n=20 \mathrm{l} \\
\text { (control } n=20 \text { ) } \\
\text { electrical }+ \text { pressure pain }\end{array}$ & $\begin{array}{l}\Downarrow \\
\text { only fibr./ } \\
\text { only pres- } \\
\text { sure pain }\end{array}$ & $\begin{array}{l}\downarrow \\
\text { only pres- } \\
\text { sure pain }\end{array}$ & \\
\hline $\begin{array}{l}\text { Lipman et al. } \\
(1987)\end{array}$ & $\begin{array}{l}\text { various organ. etiol. }\{n=27) / \\
\text { (control } n=17) / \text { thermal pain }\end{array}$ & & $\uparrow$ & \\
\hline $\begin{array}{l}\text { Langemark et al. } \\
(1989)\end{array}$ & $\begin{array}{l}\text { tension headache }(n=50) / \\
\text { (control } n=24) \\
\text { cold and heat pain }\end{array}$ & $\downarrow$ & & \\
\hline
\end{tabular}

Table 3. 1: Studies which have tested either pain threshold, pain tolerance or pain discrimination in chronic pain patients and healthy controls. 
Limiting the discussion first to only the studies with CLBP patients, it appears that the results are consistent. Pain threshold was always found to be higher, whereas the maximal pain intensity which these patients were willing to tolerate was lower. In addition, pain discrimination was decreased, indicating less capacity to distinguish between different intensities of a pain stimulus. However, the fact that pain threshold and pain discrimination on the one hand, and pain tolerance on the other, have never been measured in the same study makes it difficult to draw a definite conclusion. Moreover, pain threshold was measured by another pain stimulus as was pain tolerance. The studies which measured pain threshold in CLBP patients always used radiant heat stimuli, while the studies which measured pain tolerance used the cold pressor test. Yet, the view that CLBP patients have an increased pain threshold, a decreased pain tolerance and a diminished capacity to discriminate between different painful intensities will be adopted as a preliminary descriptive hypothesis.

The next question which can be asked is whether this preliminary hypothesis also fits the data of the studies with other chronic pain patients. Going down the table to studies which have measured experimental pain perception in patients with various other pain syndromes, the results appear to be less consistent. Three studies found an increased pain threshold (in the studly of Huskisson and Hart, this was only true for ankylosing spondylitis patients, but not for rheumatoid arthritis patients), whereas four studies found a decreased pain threshold. Only three of the studies measured pain tolerance, of which two found a lower pain tolerance in the patients group (one only non-significantly) and one a higher pain tolerance. Pain discrimination was measured in just one study, and this was found to be decreased in chronic pain patients, corresponding to the finding in CLBP patients. The eight studies will now be discussed separately in relation to their consistency with the preliminary hypothesis of increased pain threshold and decreased pain tolerance and discrimination.

The results of the study of Hazouri and Mueller are consistent with the hypothesis: a higher threshold for thermal pain was found in the patient group. However, the number of patients in this study was extremely small $(n=3)$. The study of Huskisson and Hart is only partly consistent with the hypothesis. A higher pain threshold was found for ankylosing spondylitis patients, but not for rheumatoid arthritis patients. Because the paper does not specify to what site(s) the pressure pain stimulus was applied, it is not clear whether there could have been any overlap between clinical pain site and site of experimental pain. As rheumatoid arthritis is characterized by widespread areas of pain, the possibility of overlap in this group is probably greater than for the ankylosing spondylitis patients, experiencing localized back pain.

Clearly at variance with the preliminary hypothesis is the study of Molin et al. with mandibular pain patients. Threshold and tolerance for an electrical pain stimulus were both found to be lower in the patient group. The next study, by Merskey and Evans, studied patients with clear organic causes for their pain, mainly of neurologic origin, or cancer pain. Using a pressure pain stimulus, a higher pain threshold was found, in agreement with the hypothesis.

The study of Malow et. al. with myofascial pain patients at first seems inconsistent with the hypothesis, because a decreased threshold for pressure pain was reported. However, the definition of pain threshold which the authors have employed, is clearly 
different from the definition normally used. The stimulus intensity at which a subjects scored a " 9 " on a 1.0-point scale (0-9), corresponding to the verbal label "definite pain", was considered to be the pain threshold. This probably does not correspond to the least stumulus intensity which is experienced as painful (fulfilling the definition of pain threshold). It might even be argued that it is closer to pain tolerance, or at least that it represents a level somewhere in between pain threshold and pain tolerance. Considering this, the results of Malow et. al. might not be all that inconsistent with the hypothesis. In addition, this study was the only one measuring pain discrimination in patients with chronic pain other than CLBP, and the result of this variable is consistent with the hypothesis.

A study definitely at variance with the hypothesis, at least where pain threshold is concerned, is the study of Scudds et. al. This study assessed the pain sensitivity of fibrositis (fibromyalgia) patients, rheumatoid arthritis patients and pain-free controls. Three different pain stimuli were used, two kinds of mechanical pressure pain stimuli and an electrical pain stimulus. With two of the pain stimuli (the electrical one and one of the pressure pain stimuli) only non-significant differences were found. However, with the second pressure pain stimulus, a significantly lower pain threshold was found for the fibrositis patients, whereas rheumatoid arthritis patients did not differ from controls. Pain tolerance, also only for one of the pressure pain stimuli, was lower for both pain groups.

Lipman et al. assessed pain tolerance in a group of 27 chronic pain patients with various etiologies (mostly neurologic), using a thermal pain stimulus. This has been the only study to date reporting a higher pain tolerance in chronic pain patients, once more contradicting the hypothesis. However, this might again be atributed to a problem of definition. In this study pain tollerance was defined as "the sensation experienced unequiwocally and unenduringly as pain". Because of the nature of the stimulus, this sensation was reached within a very short time, 8 seconds on average. Usually pain tolerance measures are tests of endurance, in which subjects have to tolerate pain for a relatively long period of time. Tolerance assessment in the Lipman et al. study is clearly not an endurance test.

The last study in the overview, the study of Langemark with tension headache patients, again found a result inconsistent with the lnypothesis. Pain threshold was found to be significantly lower for headache patients, both for cold and heat pain, applied to facial areas as well as to the hands. Another sudy with headache patients (patients with migraine with interparoxysmal headache, a severe form of migraine), not included in the table because no subjective measures were taken, confirmed this finding by means of the nociceptive flexion reflex. In these severe headache patients, the reflex was found to be lower than in normal subjects (Sandrini et al., 1985).

To summarize, with CLBP patients the results on pain sensitivity measures have been quite consistent. From these findings, a preliminary descriptive hypothesis has been derived, proposing an increased pain theshold, a decreased pain tolerance and a decreased pain discrimination in CLBP patients. This hypothesis now awaits confurmation by studies measuring all three variables in the same patients, and by means of the same pain stimulus. 
Next, it was explored whether the results from studies with other pain syndromes would also fit in with the preliminary hypothesis. It was shown that the results were fairly inconsistent. A number of studies were in agreement with the lyypothesis, whereas others were clearly at variance. Some of the disagreeing results can possibly be explained by inconsistencies in variable definition. However, at least three studies are definitely inconsistent with the hypothesis, all three of them in relation to the speculated increased pain threshold. These studies found a lower pain threshold for mandibular pain patients, fibrositis patients and tension headache patients. Hence, whereas the hypothesis might be appropriate for CLBP patients, it does not seem to be generizable to all chronic pain syndromes.

\subsection{A hypothetical model of pain responsiveness}

In the previous section it was demonstrated that chronic pain patients are sometimes characterized by increased pain sensitivity, and sometimes by decreased pain sensitivity when tested in the laboratory. On the basis of the results of the various studies, the preliminary descriptive hypothesis that chronic pain patients are characterized by an increased pain threshold and a decreased pain tolerance was formulated. Although this hypothesis fits the data from CLBP patients well, other pain syndromes were found to be less consistent with this formulation. Future studies should investigate which pain syndromes do, and which do not conform to this preliminary hypothesis.

Even if it is accepted that usually chronic pain patients are characterized by increased pain threshold and decreased pain tolerance, this phenomenon needs an explanation. The various models of altered pain perception in chronic pain patients which were discussed in previous sections are not in themselves capable of explaining this dual effect. They predict either increased or decreased pain sensitivity, but cannot accommodate both. Therefore, it will be proposed here that two antagonistic mechanisms are responsible for the pain responsiveness of chronic pain patients. Which mechanism will predominate is proposed to be dependent on the intensity of the experimental pain stimulus. It will further be argued that the mechanism leading to increased pain sensitivity is a psychological one, while the mechanism leading to decreased pain sensitivity is a physiological one.

Two of the models discussed in the previous sections predict increased pain sensitivity in chronic pain patients. The first is the hypervigilance/hypochondriasis model, which states that chronic pain patients are more sensitive to pain because they have a greater awareness of proprioceptive signals, and in addition consider these as more meaningful or threatening. The second model predicting increased pain sensitivity is the operant conditioning model. Verbal and behavioural pain responses (e.g. avoidance) are considered to be learned behaviours, which have become reinforced in the development of chronic pair. Both models can lead to an increased tendency in chronic pain patients to label a sensation as painful. This will be referred to as a 
tendencies can be thought of as resulting from the operation of either hypervigllance or operant learning processes separately, or from the combination of the two.

Assuming that chronic pain patients do have such response tendencies, one would expect these to operate on the whole range of stimulus intensities. Hypervigilance and hypochondriasis will exert their infuence just as powerfully on low intensity stimuli as on high intensity stimuli. And learning processes affecting pain responses will probably also be displayed at all intensity levels (except perhaps for avoidance learning, which can be hypothesized to be stronger with more painful stimuli). Considering this, the fact that at pain threshold level chronic pain patients are not hypersensitive, but instead show decreased pain sensitivity suggests that at a low pain level, the operation of response tendencies is masked by a second, antagonistic, process. The mechanism which deserves consideration for this second process is counterirritation, and its presumed neurophysiological basis DNIC. However, the perceptual mechanism based upon the adaptation level theory would also be a possible candidate for the pain decreasing mechanism. Still, there are two reasons why an explanation in terms of DNIC is more likely than an explanation on the basis of the adaptation level theory. The first reason is that the specific effect on low intensity stimulation is more easily explained by DNIC than by the adaptation level theory. The second reason is that DNIC recently was found to be able to affect pain discrimination (Talbot et al., 1989). Therefore, DNIC can offer an explanation for the decreased discriminability in chronic pain patients, which adaptation level is unable to explain.

Evidence for the proposition that DNIC more easily inhibits low intensity pain stimuli than high intensity pain stimuli comes from studies directly monitoring spinal neurons. The neuronal discharge rate of spinal nociceptive neurons increases as a function of stimulus intensity (Besson \& Chaouch, 1987). Tomlinson et al. (1983) noted that the inhibition by DNIC of neurons with low firing levels was more apparent and of longer duration than the inhibition of neurons with high levels of activity, when the intensity of the stimulus eliciting DNIC was constant. This agrees with an earlier finding of Le Bars et al. (1979) that the A $\delta$ activity of convergent neurons was inhibited by $80 \%$ when this activity was at threshold level, but only by $20 \%$ when the activity was suprathreshold. The reason for a more powerful inhibition of low levels of neuronal activity is further elucidated by Schouenborg \& Dickenson (1985). They observed that the main effect of DNIC was to decrease the nociceptive discharge rate of a target neuron by an almost constant number of spikes, independent of the initial level of activity (number of spikes) of these neurons. As a result, the percentage of inhibition was larger when the initial nociceptive activity was low than when it was high.

From these observations it can be concluded that low intensity pain stimuli are more easily inhibited than high intensity pain stimuli. In combination with one of the fundamental postulates of DNIC, namely that the amount of inhibition is directly related to the strength of the stimulus inducing DNIC, this leads to the conclusion that the balance between the two stimuli (the DNIC inducing stimulus, and the target stimulus) determines whether inhibition is apparent, and how strong it will be. Or as Le Bars et al. (1979) have stated: "... when two simultaneous noxious stimuli are applied on two distant parts of the body, the pool of convergent dorsal horn units 
related to the slighter stimuli is inhibited".

The more prominent inhibitory effect of DNIC on low intensity pain stimuli has not only been demonstrated at a neuronal level, but also in a human counterirritation study. Plaghki at al. (1990) used the cold pressor test as a counterimitation stimulus, and tested it against the perception of noxious laser heat pulses. The inhibitory effect of the counterirrtation stimulus was only apparent with laser heat pulses at or near threshold level. Non-noxious stimuli and stimuli of a higher painful intensity were not affected. This directly demonstrates that counteriritation is most effective on low intensity pain stimuli (near pain threshold). As this has been the only study so far comparing the counterirritation effect on the perception of different pain intensity levels, replication is required.

However, the intensity of the target pain stimulus does not appear to be the only relevant factor determining whether inhibition by a second pain stimulus will occur. Studies with the nociceptive flexion reflex have found that not only the threshold of the RIII reflex can increase with counterimitation, but that also maximal reflex amplitude can diminish (Willer et al, 1984). The maximal reflex only occurs with high intensity stimulation. It can be argued that besides intensity, additional factors play a role in determining the efficacy of counterirritation. These factors could be spatial and temporal distribution of a stimulus. Willer et al. (1984) have stated that: "The conditioning stimulus (= the stimulus eliciting DNIC) requires spatial and temporal summation much greater than the duration and strength of the conditioned stimulus ( $=$ the target stimulus) to be fully effective". Even though the intensity of the stimulus necessary to elicit a maximal reflex was perhaps higher than that of the counterirritation stimulus, spatial and temporal summation was much less. The counterirritation stimulus was of long duration, whereas the stimulus which elicited the reflex lasted only 20 msecs. Besides, the input from the conditioning stimulus (immersion of the hand in hot water) had a much wider temporal distribution than the restricted input from the electrical stimulus. Thus, the critical factor whether or not DNIC is effective seems to be the balance between the intensity, duration and temporal distribution of the eliciting stimulus, versus that of the target pain stimulus.

In chronic pain patients, the counterirritation stimulus is supposed to be their clinical pain. The above considerations would lead one to speculate that whenever chronic pain is more intense and more widespread than the experimental pain stimulus, the inhibition of the latter will be more pronounced. Weak, short and restricted experimental pain stimuli are therefore predicted to be more powerfully inhibited than stronger, longer and more diffuse ones. Obviously then, the effect of DNIC will be stronger on pain threshold stimuli than on pain tolerance stimuli. The latter are not only more intense, but usually also of llonger duration (depending on how tolerance is assessed). Also, heat stimuli to restricted areas will probably be more easily inhibited than, for instance, pressure pain stimuli to more widespread areas. Finally, the intensity of clinical pain a patient experiences at the moment of testing is crucial, as the amount of inhibition of the target pain stimulus is dependent on the balance between chronic pain on the one hand and experimental pain on the other.

In conclusion, though rather tentative at the moment, DNIC seems to be able to 
offer an explanation for the finding that decreased pain sensitivity is only found at low intensity pain levels. Why the alternative mechanism, based upon the adaptation level theory would influence pain threshold more than pain tolerance is not directy obvious. One could speculate that if the experimental pain stimulus exceeds the clinical pain level, the latter cannot serve as an adequate reference point anymore, and the adaptation level effect is abolished. No confirmatory data for this suggestion are available at the moment. Therefore, the DNIC interpretation is preferable for now.

Moreover, there is still another reason for DNIC to be the most plausible mechanism, namely the finding of decreased discriminability. Again, the adaptation level theory does not seem to offer an adequate explanation for decreased pain discrimination. By contrast, recent findings have suggested that DNIC does in fact influence discrimination of pain signals. Talbot, Duncan \& Bushnell (1989) have speculated that: "If WDR (= convergent) cells do play an important role in discriminative aspects of nociception, then the inhibition of these cells by DNICs should detract from an individual's capacity to discriminate between different intensities of noxious stimuli." This hypothesis was subsequently tested by the same authors. Subjects were presented a reaction time (RT) discrimination task with and without a concurrent counterirritation stimulus. In the RT task, discrimination was defined as the time needed by a subject to detect a small change in a painful heat stimulus. The stimulus started from an initial (painful) level, and than gradually increased slightly. When the subject could detect this increase, he/she had to press a button. The time from onset of the intensity increase to detection was the dependent variable. It was shown that in combination with a counterirritation stimulus, subjects needed significantly more lime to detect the change in intensity. Hence, it seems that counterirritation can intluence pain discrimination. Whether this finding can be generalized to other discrimination tasks (more resembling the tasks which have been used in chronic pain patients) remains to be determined.

Figure 3.2 summarizes the proposed relationship between stimulus intensity and pain sensitivity in chronic pain patients. Several points can be emphasized. Because pain threshold is higher, and pain tolerance is lower, the slope of the pain perception curve for chronic pain patients is steeper. The point of intercept between the two curves is the hypothetical point where the pain decreasing and pain increasing mechanisms are in equilibrium. Below that point, DNIC has a stronger impact on pain perception in chronic pain patients than response tendencies. Above that point, DNIC has lost most of its efficacy, and response tendencies determine the pain response.

The model also implies that whether stimulus intensities between the pain threshold and pain tolerance are rated as more or less painful by chronic pain patients compared to the ratings of control subjects, depends on the position of objective stimulus intensity on the $x$-axis. Thus, a stimulus with an intensity close to pain threshold will be scored as less painful and a stimulus with an intensity close to pain tolerance as more painful. Stimuli at intermediate intensities will be scored as approximately equally painful by chronic pain patients and healthy controls. The path of the curve below the level of pain threshold has deliberately been omitted, because little is known about the 
sensitivity for non-nociceptive signals in chronic pain patients. Predictions can be made in either direction (increased or decreased sensitivity). The next section will deal with this topic in more detail.

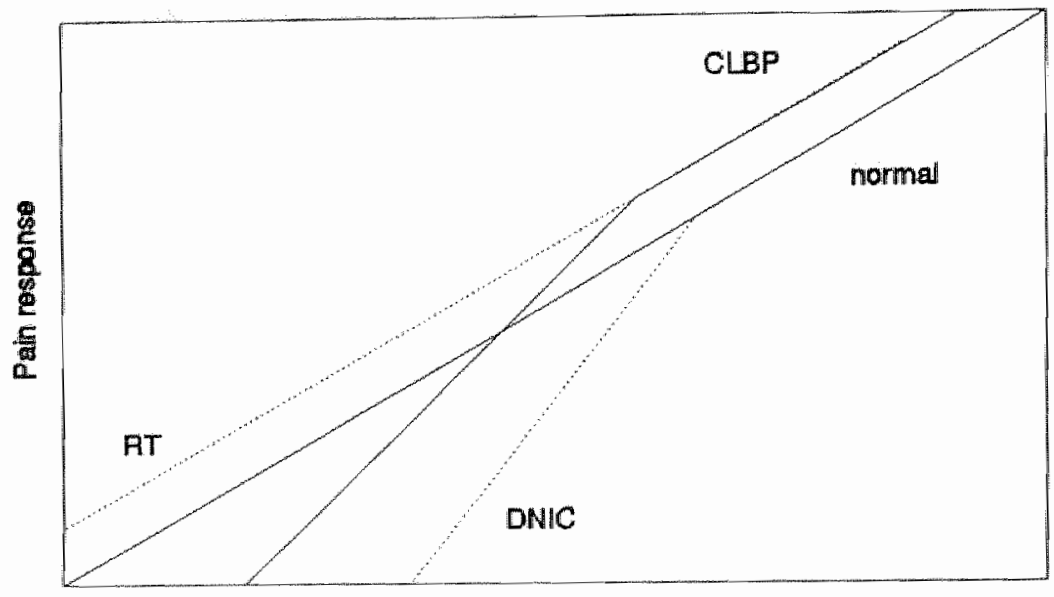

Intensity pain stimulus

figure 3.2: A schematic representation of pain perception in chronic pain patients and controls as a function of stimulus intensity. Response tendencies (RT) have a pain increasing function, DNIC a pain decreasing function.

In this dissertation, the model will be tested. If will be attempted to establish whether the model accurately describes the pain perception of CLBP patients, not only for pain threshold and pain tolerance, but also as regards stimuli at an intermediate intensity level.

\subsection{Sensitivity for non-painful interoceptive and tactual signals}

In the previous section the topic of sensitivity to non-painful tactual signals was briefly touched upon. Whether the sensitivity to non-nociceptive tactual signals is changed as a result of chronic pain has hardly been tested. Most studies so far have directed their attention to the perception of noxious stimuli only. Therefore, in the model presented in the previous section, no attempt was made to draw the path of the 
curve below pain threshold. Three of the models discussed in this chapter do predict altered pain sensitivity for non-painful tactual or interoceptive signals, or can at least accommodate for such differences. However, the models make predictions in opposite directions.

The first model which predicts altered sensitivity for non-nociceptive internal signals is the hyperwigilance/hypochondriasis model. Increased attention to all kinds of interoceptive signals leads to heightened sensitivity for both noxious and non" noxious signals. The operant learning model of chronic pain also aliows for increased sensitivity for non-nociceptive interoceptive signals in chronic pain patients. Generalization of complaining behaviour can go so far that it is transferred to other bodily sensations besides pain. By trial-and-error learning the patient has discovered how to get maximal reinforcement from this or her environment. Complaining about more than only chronic pain will elicit more positive reinforcement.

The prediction of decreased sensitivity for non-nociceptive tactual signals can be made from the DNIC model. DNIC has been shown to inhibit all activity of convergent neurons, whether elicited by noxious or non-noxious stimulation. Thus, both $\mathrm{A} \alpha-$ (non-noxious) and $A \delta$ - and $C$-fiber (noxious) responses are inhibited, although the inhibition of $A \alpha$-fiber activity was shown to be less than that of $A \delta$ - and $C$-fiber activity (Le Bars et al., 1979b).

The little research which has been conducted on the issue of chronic pain and sensitivity for non-nociceptive internal signals, has not been conclusive. For both increased sensitivity and decreased sensitivity some support has been found. Schmidt et al. (1989) have demonstrated that CLBP patients report more non-specific bodily sensations in both exertion and rest conditions in comparison to a healthy controll group. Complaining about interoceptive signals is increased, consistent with both the hypervigilance/hypochondriasis and operant conditioning model. On the other hand, Seltzer \& Seltzer (1986) found a higher tactile two-point discrimination threshold in chronic pain patients (with unspecified diagnoses). Thus, their sensory sensitivity for tactile stimuli seemed to be decreased. Finally, Naliboff et al. (1981) did not find any differences between CLBP patients and controls in detection threshold for a heat stimulus.

In sum, only few studies have measured non-nociceptive tactual sensitivity in chronic pain patients. In order to be able to draw a more definite conclusion, more studies are needed.

\subsection{Questions addressed in this dissertation}

The preliminary descriptive hypothesis, formulated in section 3.7 (pain threshold is higher, pain tolerance is lower, and pain discrimination is less in chronic pain patients) requires confirmation. From a review of previous studies, this formulation seems to fit the data obtained from CLBP patients better than those obtained from chronic pain patients in gemeral. However, even for CLBP patients, the hypothesis is still preliminary. It was formulated post hoc, and has never been tested directly by measuring pain threshold and pain tolerance by means of the same stimulus in the 
sume patients.

A major objectrve of this dissertation is to thest the validity of the preliminary hypothesis. This will be restricted to its application to CLBP patients only. If the hypothesis is found to hold for this group, it can later perhaps be extended to include patients with other pain syndromes. The prediction that pain threshold is higher for CLBP patients and pain tolerance is lower, is tested in the studies reported in the sections 5.1,5.3 and 5.7. These studies have employed a pressure pain stinulus, an electrical pain stimulus, and both an elecrical and a pressure pain stimulus, respectively. The hypothesig that CLBP patients do not discriminate as well as controls between different intensity levels of an electrical pain stimulus is tested in the study reported in section 5.6 .

The next question which is addressed is whether the model proposed in section 3.8 is an accurate description of the way in which chronic pain patients percerve experimental pain stimuli. Again, the studies related to this question only concems CLBP patients. The model goes beyond the descriptive level of the preliminary hypothesis. Firstly, speculations are made about the cause of the divergent effects on pain threshold and pain tolerance. It is hypothesized that this depends on intensity of the pain stimulus. Some additional predictions can be deduced from this speculation. When pain stimuli of graded intensity are applied, the pain ratings of chronic pain patients are higher or lower as compared to a control group according to stimulus intensity. Thus, in a series of stimuli of increasing intensity, CLBP patients will at first rate the stimuli as less painful than controls, but later on they will rate them as more painful. Stimuli of intermediate intensity are rated as equally painful by CLBP patients and controls. In addition, the slope of the pain intensity curve is steeper for CLBP patients. The study presented in section 5.3 was set up to test this prediction.

The second reason why the model goes beyond a descriptive level is that the two antagonistic mechanisms which are supposed to determine the pain sensitivity of chronic pain patients are specified: response tendencies on the one hand and DNIC on the other. In this dissertation it is tested whether these mechanisms do in fact play a role in the altered pain sensitivity of chronic pain patients. In section 5.3 it is examined whether the operation of response tendencies in CLBP patients can be demonstrated. Sections 5.6 and 5.8 assess the operation of counterimitation in healthy volunters. In section 5.6 the intluence of a pressure pain stimulus on the detection threshold, pain threshold, pain tolerance and pain discrimination of an electrical stimulus is measured. The purpose of this study is to establish whether a counterirritation stimulus indeed affects pain threshold more than pain tolerance, and whether pain discrimination is also diminished by counterirritation. Exploratory, the effect of counterirritation on detection threshold is measured. In section 5.8 it is tested whether the finding that a counterimitation stimulus depresses the Rill reflex can be replicated. If so, than measuring the RIII reflex in chronic pain patients is worthwhile. This has been performed in the study reported in section 5.9 .

The aim of the last study of this thesis (section 5.9) was to find a conclusive answer to the question whether or not DNIC is induced by chromic pain, and that it is the mechanism responsible for the decreased pain threshold in CLBP patients. The study tests whether clinical pain in general (acute and chronic) is able to activate 
DNIC. Therefore, pain-free volunteers are tested against CLBP patients and patients with acute post-operative pain. Pain sensitivity is assessed by means of subjective pain threshold measurement and measurement of the RIII reflex. To test the operation of DNIC more directly, a naloxone condition is included. As was discussed above, DNIC is suppressed by the administration of this opioid antagonist. If DNIC is the mechanism responsible for an increased pain threshold in the two pain groups, the administration of naloxone will antagonize this threshold increase.

An additional question addressed in this dissertation is whether CLBP patients have an altered sensitivity for non-painful tactle sensations. As noted in the previous section, little research has been performed in this area. Section 5.3 has measured the detection threshold for an electrical stimulus. In addition, the tactile two-point discrimination threshold of CLBP patients was tested in the study reported in section 5.5 . 



\section{CHRONIC PAIN AND HABITUATION TO EXPERIMENTAL PAIN STIMULI}

\subsection{Introduction}

The previous chapter was devoted to the responsiveness of chronic pain patients to experimental pain stimuli. In this chapter the issue will be extended to the responsiveness to repeated pain stimuli. Do chronic pain patients show different habituation patterns to such repeated pain stimuli? This question originates in the fact that chronic pain, by definition, is characterized by a lack of efficacious habituation of the experience of pain. It has been suggested that an inability to habituate to painful experiences might be a risk factor for developing chronic pain, or a serious handicap in the process of rehabilitation (Brands \& Schmidt, 1987). In the acute pain phase, the inability to adjust to a recurrent or relatively enduring pain episode could promote the establishment of pain behaviours.

Habituation to pain stimuli can be assumed to depend on the same basic processes as habituation to all other sorts of stimuli. Therefore, this chapter will begin with a discussion of the hypothesized mechanisms of habituation in general. Several models have been proposed, and the three most influential ones will be discussed. Next, evidence that organisms are usually capable of habituation to acute pain stimuli will be presented. In the past, it has been proposed that habituation to non-noxious stimuli. occurs readily, but that it is not possible to habituate to aversive and painful stimuli. Experimental findings have refuted this assumption.

The degree and rate of habituation are determined by several factors. The most important ones will be presented, and their influence on habituation of the experimental and chronic pain experience will be discussed. Finally, emperical evidence for a decreased habituation to experimental pain stimuli in chronic pain patients is reviewed. Potential deficits of habituation to painful experiences can be expected to extend to experimental pain stimuli too. So far, few studies have compared the degree of habituation of subjective and physiological responses to repeated experimental pain stimuli between chronic pain patients and controls. An overview is presented.

\subsection{Models of habituation ${ }^{1}$}

Although habituation is a very common phenomenon, affecting most behavioural and physiological response systems, its theoretical basis is still speculative. Habitua-

\footnotetext{
'This section is partly based on a paper by Arntz \& Peters 19901.
} 
tion is ustally considered as the most primitive form of leaming, characterized by a decrement of responsiveness to monotonously repeated or continuous stimulation. It is not due to receptor adaptation or effector fatigue, which is demonstrated by the fact that after a change of stimulus characteristics, the response can reappear in full magnitude (dishabituation). Habituation can be found in the most primitive of animals, even in protozoa. Whether the mechanisms of habituation are the same in these primitive animals as in more evolved wertebrates can be questioned. In invertebrates, no involvement of higher nervous system structures is required for habituation to occur. However, in more evolved species, the involvement of higher nervous system structures in the process of habituation has been implicated. Moreover, it can also be questioned whether within species, all response decrements are due to a single habituation process. For instance, two of the models which will be discussed below postulate the involvement of the brain in habituation, but at least some responses (e.g. the wilhdrawal reflex) do not require intact connections with the brain for habituation to occur. Habituation of these responses therefore have to be spinally mediated.

Usually, a distinction is made between short-term versus long-term habination. In the former case, response decrement persists only for several seconds or minutes. After the interuption of stimulation, the effect soon disappears and spontaneous recovery of the response is seen. However, a decline in responsiveness can also persist for longer periods of time, even for days. This form of response decline is called long-term habituation. The explanation of short versus long-term habituation may require the supposition of different mechanisms. Below, the three most influential models of habituation will be outlined. It will be argued that not all of these models are equally capable of explaining both kinds of habituation.

\section{a. The dual-process model}

The dual-process model of habituation was formulated by Groves \& Thompson (1970) to account for the observation that after repeated stimulation both habituation and sensitization can be observed. The model was derived mainly on the basis of studies on spinal reflex activity in the cat. Only short-term habituation is observed with this reflex (Thompson \& Spencer, 1966), and therefore the model specifically deals with short-term habituation processes. As the name already conveys, the dualprocess model assumes the operation of two discernable processes, one decreasing responsiveness (habituation) and the other increasing responsiveness (sensitization). In addition, it is suggested that the process of habituation and the process of sensitization originate in two distinct physiological systems: a direct stimulus-response pathway (the S-R pathway) and a "state" system, consisting of the collection of pathways, systems and regions determining the general arousal level of the organism. The process of habituation is assumed to operate on the direct S-R pathway: repeated stimulation will cause a decline in the transmission between input and output systems. The process of sensitization occurs through the state system. When arousal increases, transmission between in and output systems is facilitated. The habituation process in the S-R pathway, and the sensitization process in the state system occur independently of one another. A stimulus presentation can influence both systems simultaneously. It will always activate the S-R pathway and sometimes will alter the state of the 
organism. The strength of a response to a repeated stimulus is ultimately dekrmined by the net outcome of the two processes of habituation and sensitization. The dual process model locates the neurophysiological basis of habituation and sensitization at the level of spinal interneurons. Repeated stimulation is assumed to alter the firing frequency of these neurons.

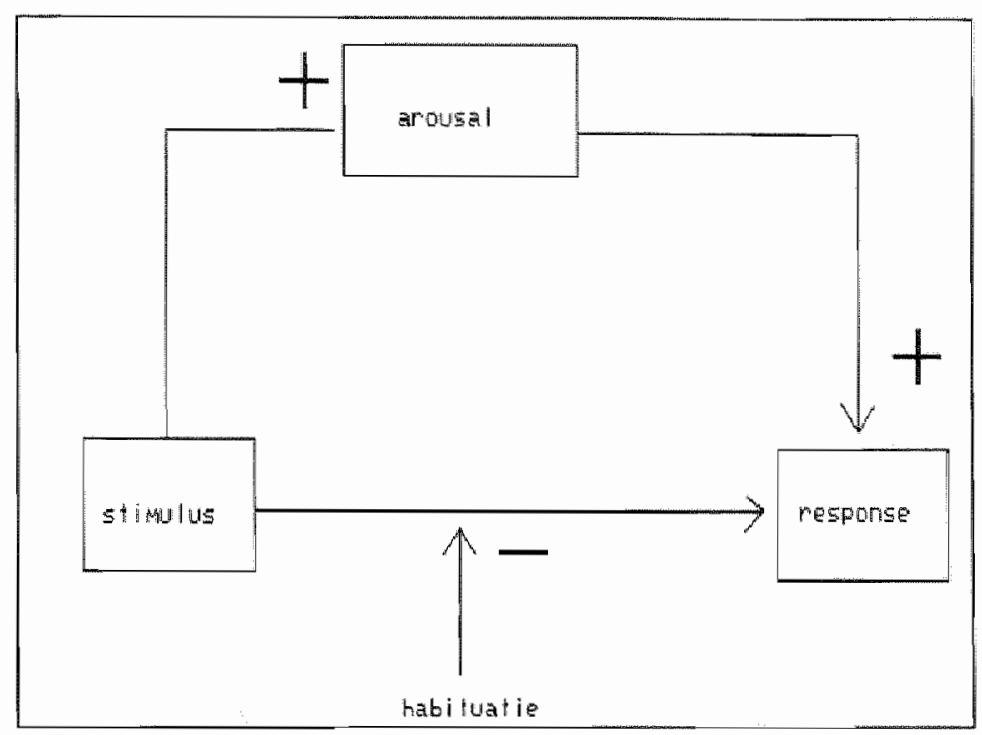

fig 4.1: A schematic representation of the dual-process model of habituation. Habituation exerts its influence on the direct stimulus-response pathway, whereas sensitization occurs through the arousal system.

The dual-process model is well capable of explaining the occurrence of the opposite processes of habituation and sensitization. However, it only accounts for temporary learning processes and cannot explain long-term habituation or long-term sensitization. Moreover, the data on which this model is based, are obtained mostly from studies with spinalized animals. Hence, it can explain changes in spinal reflex activity adequately, but ignores the effect that higher nervous system processes have on habituation.

\section{b. Inhibition models}

A second class of models assumes that habituation depends on the operation of an active inhibitory influence of higher brain centers (Sokolov, 1960; Gray; 1975). The model of Sokolov is the most well-known in this class. It was formulated to account for such phenomena as the recovery of responsiveness which occurs after the alteration of some characteristic of an already habituated stimulus. Sokolov proposes that such dishabituation can be explained by the operation of a kind of neural "comparator". Presentation of a stimulus will give rise to the formation of a neuronal 
model, containing essential information about such aspects as duration, strength and effect of the stimulus. When subsequent stimulus presentations match this model, the "comparator" inhibits the response to the stimulus. As in the previous model, the existence of an arousal system is assumed. This system ultimately determines the strength of the reaction to a stimulus. The arousal system is inhibited when a match between a stimulus and the neuronal model occurs. A mismatch results in less inhibition of the arousal system and consequenily in increased responsiveness. Repeating an identical stimulus promotes the formation of a more closely matching model, and thus in a greater degree of inhibition (habituation). When a different stimulus is presented suddenly, a mismatch will occur, causing dishaoituation. If a stimulus is unpredictable in terms of occurrence or intensity, no appropriate model can be formed and habituation will be absent. Sokolov located the principal components of his model at higher nervous system levels. The arousal system would be primarily a function of the reticular formation, whereas the comparator was initially thought to be located in neocortical structures. Later, the limbic system and especially the hippocampus were implicated to play a role in the comparison processes (van Olst et al., 1980).

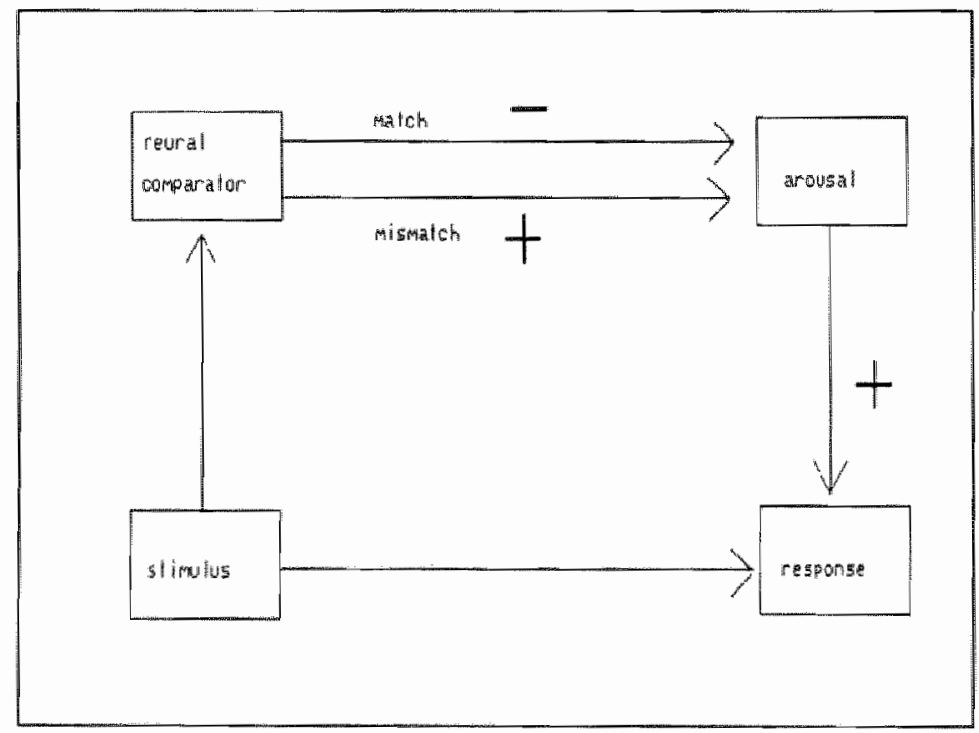

fig. 4.2: A schematic representation of Sokolov's inhibition model of habituation. A stimulus representation is fed to the comparator. A match results in inhibition of arousal and a decrease in responsiveness. Mismatches do not inhibit arousal. Repetition of a stimulus will llead to formation of a more closely matching model.

Compared to the dual-process model, inhibition models are more capable of explaining several habituation phenomena. Sensitization is explained in the same way 
as in the previous model, namely by means of increased arousal. Long-tern habiwation can be explained by assuming that the neuronal model can persist for longer periods of time. In addition, inhibition models are particularly suited for explaining the reappearance of previously habituated responses after changing stimulus characteristics, as well as the phenomenon that a response can occur when a stimulus which is expected does not occur. Both stimulus change and stimulus omission result in a mismatch with the neuronal model. However, several findings remain which cannot be explained by inhibition models. For instance, the model predicts that every stimulus change, irrespective of the direction of the change, causes increased responsiveness. However, it has been shown that deviations of the stimulus towards higher intensities result in stronger reactivity than deviations towards lesser intensity. Thus the system seems to be especially sensitive to "dangerous" mismatches (Rachman \& Lopatka, 1986; Arntz \& van den Hout, 1988). Moreover, not every stimulus change will result in dishabituation, even if it is detected by a subject.

\section{c. Information-processing models}

More recently, models of habituation based on memory and information-processing theories have been proposed (Wagner, 1976; Wagner, 1979; Wagner, 1981; Ohman, 1979). Several specific versions of these models exist, which have in common that they are based on formulations from the field of cognitive psychology and emphasize the fact that an organism has only a limited capacity for information processing. One of the models (that of Wagner) will be selected for a more detailed discussion. In the model of Wagner, the assumption is made that the mental representation of a stimulus can appear in three different states: in an inactive state (long-term memory), in a state of primary activation (working memory or rehearsal) and in a state of secondary activation. The primary and secondary activation states together constitute short-term memory. Exposure to a stimulus will bring the stimulus representation from the inac. tive state to the primary activation state. Afterwards, the activated representation of the stimulus "decays" to the secondary activation state, and eventually back to the inactive state. A stimullus representation in the primary activation state will give rise to responses and (in humans) conscious experience. In the secondary activation state, the representation is temporarily inaccessible for primary activation. If a stintulus is presented shortly after a previous stimulus presentation, the representation will still be partly in the secondary activation state, and a smaller part of the stimulus representation is available for primary activation. Consequently, the responses to the stimulus will be smaller. This process is proposed to form the basis of short-term habituation.

A stimulus will also be less likely to enter a state of primary activation when it is already represented in the short-term memory ("primed"). Priming can be based on retrieval of a representation from long-term memory. This process forms the basis of long-term babituation. Contextual cues can become conditioned stimuli (CS) to the originally unconditioned stimulus (UCS) if they are predictive of its occurrence. The contextual CS can bring part of the UCS representation directly from the inactive to the secondary activation state (priming), which means that a smaller part of the tolal UCS representation is available for primary activation. Because the response strength 
depends on the amount of UCS representation in the primary activation state, the responses will be smaller with conditioning than without conditioning. Primary activation, or rehearsal, may also be affected by other factors such as arousal, affective tone and unexpectedness of a stimulus. Unexpected stimuli are supposed to be rehearsed more than expected stimuli. A schematical overview of the model is presented in figure 4.3 .

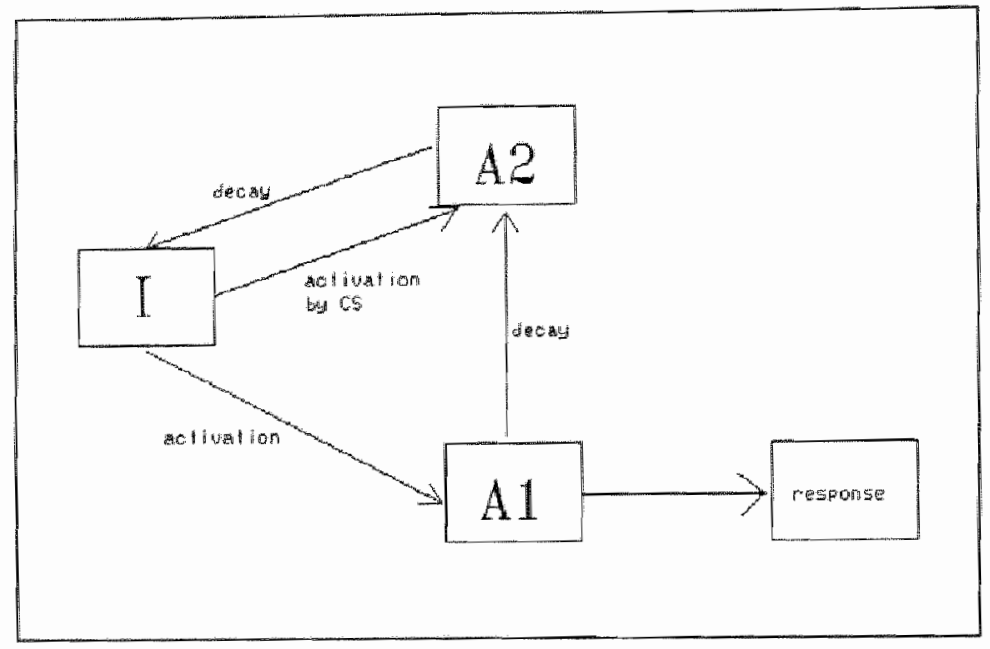

figure 4.3" A schematic representation of Wagner"s information-processing model of habituation. By direct activation, a stimulus representation is transferred from the inactive state (1) to the state of primary activation (A1). Subsequently it will decay through the state of secondary activation (A2) back to the inactive state. Context generated retrieval brings the stimulus representation direcrly from the inactive state to $\mathrm{A} 2$.

Information-processing models seem to have more explanatory power than the two previously discussed models. It can explain both short and long-term habituation, and also the fact that some stimuli habituate less than others, or cause more dishabituation. As was discussed above, the amount of rehearsal of a stimulus determines the response. An unexpected stimulus is rehearsed more, and this applies also to affectively toned events. This explains why departures from expectancy towards higher stimulus intensity elicit more dishabituation than departures towards lower stimulus intensity. And although Wagner"s model has been formulated for automatic stimulus processing, it allows for the possibility that controlled processing of information takes place. For instance, attentional processes can start an active "search" of the secondary activation state, and promote the transition of stimulus representations in the secondary activation state to the primary activation state. 


\subsection{Habituation to experimental pain stimuli}

Sokolov has argued that aversive stimuli, amongst which is pain, are resistent to habituation. This assumption constitutes part of his formulations concerning orienting and defensive responses (Sokolov, 1963). Orienting responses (OR's) are responses to sudden and unexpected stimuli. They interrupt ongoing activity and facilitate stimulus reception. According to Sokolov, characteristic of OR's is that they are non-specific, occurring regardless of stimulus quality and intensity, and that they habituate rapidly with repeated stimulation. Defensive responses (DR's) are responses which occur only after intense stimuli. DR's are aimed at reducing the impact that a stimulus has on an organism, or at flight from the stimulus. In contrast to OR's, DR's show little or no habituation and may even intensify with stimulus repetition.

Experimental research, however, has convincingly demonstrated that responses to pain stimuli do habituate after repetition of these stimuli, refuting Sokolov's claim. Overall, it appears that subjective, behavioural and plyysiological correlates of pain can habituate, although differences in the degree and rate of habituation of the various variables have been found. Behavioural variables of pain have predominantly been measured in animal research, while subjective pain measurements and physiological correlates of pain have been used in human studies. The overview of studies presented below only includes research performed with human subjects.

A frequently used pain stimulus in habituation research has been electrical shock, probably because of its good physical reproducibility, the ease of application and its harmless nature, even after repetition. Habituation to electrical stimuli was shown to occur in the studies of Condes-Lara et al. (1981), Bromm \& Scharein (1982), Ernst et al. (1986), and Collier et al. (1990). Condes-Lara and colleagues demonstrated that several physiological variables, as well as the subjective assessment of pain, decreased during 120 minutes of constant painful tooth stimulation. Four different physiological variables were assessed, evoked potentials (EP), electrocardiogram (ECG), myographic measurement (EMG) and skin conductance (SCR).

Bromm and Scharein looked at almost identical variables (however, electro-oculogram (EOG) instead of ECG) and measured both short and long-term habituation. An electrical stimulus of constant intensity was applied to the skin 40 times within a session, and sessions were repeated 4 times at two days intervall. It was found that only peripheral physiological correlates of pain (EOG, EMG and SCR) habituated on both the short and long-term, whereas subjective pain experience and EP did not habituate, neither within nor between sessions.

Ernst et al. only found short-term habituation (within session) using continuous electrical dental stimulation. Two 2-hour sessions - one week apart - took place, during which subjects underwent 13 determinations of pain detection threshold and pain discomfort threshold ("the intensity of stimulation that elicits a sensation which, if persisting, would prompt the subject to take aspirin"). During the first hour of each session, continuous electrical stimulation was applied. Thereafter, no stimulation was given anymore, except for the threshold determinations. It was shown that under these conditions both pain detection and pain discomfort threshold habituated, although the effect was stronger for the lower threshold. During the first hour of the session, the 
thresholds showed an ongoing increase which stabillzed during the second hour. No habituation beween the two sessions was found.

Collier et al. used a less extensive stimulation period. They applied 10 electrical stimuli of constant intensity to the fingers and measured pain threshold and severe pain threshold before and after this series. Even with so few stimuli, they were able to show that habituation occurred, at least in a subgroup of subjects. Other subjects showed signs of sensitization. However, the two pain thresholds always changed in the same direction. Dimitrijevic et al. (1972) measured the habituation of a nociceptive reflex (the flexion reflex of the tibialis anterior muscle) elicited by electrical stimulation to the foot. At threshold level the reflex soon habituated so that no response was ellicited anymore. At a lewel moderately above threshold, only stimulation at regular intervals produced habituation. With variable stimulus interval times, habituation did not occur or was minimal. If high intensity stimuli were given, there was no habituation, even after long series of regular stimulation.

Not only habituation to electrical stimuli has been found, habituation to hot and cold water pain has also been demonstrated. Glaser et al. (1959) found long-term habituation of the blood pressure response and the subjective pain response after immersion of the hand in hot water for 6 times a day for a total of 9 to 15 days. Habituation to hot and cold pain simultaneously was also possible, when subjects immersed their hand alternatingly into hot and coldwater. Long-term habituation of the blood pressure response to cold water pain was also found by LeBlanc \& Potvin (1966). They repeated the cold pressor test twice daily for 19 successive days. Again, blood pressure responses decreased during successive trials. Even more trials were performed by Strempel (1976), who repeated the cold pressor test 7 times dailly, for 21 days on a row. This time, long-term habituation was found in only part of the subjects. Another (smaller) part of the subjects did not habituate even after so many repetitions of the painful stimulus.

In conclusion, habituation to experimental pain stimuli has been shown to occur both on a subjective level and on a physiological level. However, studies assessing individual responses also demonstrated that often not all subjects habituated. Moreover, there have been differences between the studies regarding the degree of habituation of the various variables.

\subsection{Factors influencing habituation to pain stimuli}

Studies with non-aversive stimuli have shown that the rate and degree of habituation depend on such factors as number of exposures to a stimulus, stimulus intensity, stimulus duration and inter-stimulus interval time (Graham, 1979; Jackson, 1974). The same factors will most likely influence habituation to pain stimuli. But there are also other factors, unrelated to stimulus characteristics, which have been shown to influence habituation. Of major umportance is the significance of a stimulus to the organism.

Indications were found that the habituation to meaningful stimuli (including stimuli to which a response has to be made) occurs more slowly than to meaningless stimuli, 
and that there is no long-term habituation to meaning ful stimuli (Packer \& Siddle, 1987). Pain is an inherently meaningful stimulus, it signals danger and physical damage. Nevertheless, as has been described above, pain respones can habituate, even on the long-term. This might be due to the fact that pain is not invariably a signal of tissue damage or threat of tissue damage. For instance, subjects participating in a laboratory experiment are conscious of the fact that they will not be exposed to dangerous and life-threatening stimuli. Moreover, a response to the presented pain stimuli is usually not required of the subject, further diminishing their meaning. Habituation to pain stimuli is therefore possible and will even be functional. Habituat ing to pain stimuli will make pain less bothersome, and enhances an organism's capacity to engage in other activities. However, is it possible to habituate to clinical pain? Clinical pain is usually more threatening to an individual than experimental pain and thereby not easily becomes meaningless. Still, it can be expected that after a certain period of time even clinical pain loses its meaning. In the case of nonmalignant chronic pain there is no signalling function of the pain, the patient does not have to take action to avert a danger. But also in the case of malignant pain, endless repetition of the warning that there is "something wrong" within the body does not provide new information.

In humans, symbolic meaning and perceived threat are probably more important than actual stimulus meaning and threat. Innocuous stimuli, perceived by a subject as very threatening, will be more resistant to habituation than potentially harmful stimuli which are not perceived as threatening. The same stimulus can have a different meaning to different subjects, depending on how it is cognitively interpreted. Some people tend to interpret pain stimuli in a "catastrophic" way, a maladaptive strategy which has been shown to increase the painfulness of experimental pain stimuli (Spanos et al., 1981). In addtion, substantial cultural differences were found in the meaning of pain to an individual (Zborowski, 1952). This can be ascribed to early learning experiences. Stembach \& Tursky (1965) have shown that culturally detemined attitude towards pain can influence habituation of the psychophysiological responses to repeated pain stimuli. Anglo-american women whose ancestors were of British descent, and whose parents and grandparents were born in the United States were more indifferent to pain than women whose parents had come as immigrants to the United States, and who were of Irish, Italian and Jewish descent. The Anglo-american women also showed the fastest habituation of the palmar skin potential response to pain.

A second important factor influencing the habituation to pain stimuli is attention. Attention and habituation mutually influence each other. Habituation allows an organism to attend to new or more significant events. Without habituation, an. organism would be llooded by numerous intro- and exteroceptive stimuli. But not only does habituation make the distraction of attention possible, conversely, the distraction of attention promotes the occurrence of habituation. Attention directed away from a stimulus diminishes the initial responsiveness to that stimulus, and also enhance the decline of responsiveness on subsequent trials. Iacono \& Lykken (1984) have shown that subjects who were instructed to distract themselves from painfull stimulation and to concentrate on a movie, had smaller SCRs to the pain stimuli initi- 
ally, and habituated more rapidly. Attention directed at noxious stimulation, or on the physical consequences of these stimuli (at interoceptive signals) retards habituation (Scheier et al., 1983). Another experiment by Arntz et al. (1991b) demonstrated that in conditions in which anxiety and attention were varied independently, attention did influence habituation, whereas anxiety did not. Subjects were assigned to one of four conditions: (1) high anxiety, attention directed at the pain stimulus, (2) high anxiety, attention directed away from the pain stimulus, (3) low anxiety, attention directed at the pain stimulus and (4) low anxiety, attention directed away from the pain stimulus. In both low and high anxiety conditions, when attention was focussed on the pain, no habituation to 20 electrical pain stimuli was found. However, when attention was distracted from the pain stimuli, habituation did occur, independent of level of anxiety.

The discrepancy between expected pain intensity and experienced pain intensity is another factor which can determine the process of habituation. According to the inhibition models of habituation, effective inhibition only occurs when the experience of a stimulus matches the neuronal model (the expectation) an organism has formed of it. The information-processing model of Wagner also states that a stimulus which is unexpected will elicit a stronger response than expected stimuli. Departures from expectation can occur, for example, in intensity. When an incorrect expectation of pain intensity has been formed, inhibition and information-processing models expect such a mismatch to result in dishabituation. Empirical findings have shown that especially departures in the direction of higher intensity levels have a prominent effect. It has been confirmed experimentally that indeed underpredictions of pain do have a dishabituating effect, whereas overpredictions of pain do not lead to dishabituation (Arntz, 1989; Arntz \& Lousberg, 1990; Arntz et al., 1990). Rather, an overprediction seems to be functional: it might promote the inhibition of pain (Epstein \& Clarke, 1970). However, overprediction of pain also poses a potential danger for pain habituation. If pain stimuli are avoided because of increased anxiety, the occurrence of habituation is prevented.

In sum, the meaning of pain, attention to pain, inaccurate predictions of pain and avoidance of pain determine whether and to what degree habituation to pain stimuli will occur. These observations are also clinically relevant. For instance, the meaning which a patient attaches to his/her pain and the attitude towards this pain could promote the chronicity of pain. In this context, the discussion in the previous chapter on hypochondriasis might be recalled. Patients with hypochondriacal tendencies are inclined to altach exaggerated significance to all kinds of bodily signals. The assugnment of excessive significance to pain signals can hinder the habituation to these signals. Moreover, the other component of hypochondriasis, increased attention to somatic signals, will likewise obstruct habituation. Hence, decreased habituation to painful stimulation would be expected in hypochondriacs. Unfortunately, no studies have been performed to test this hypothesis.

Incorrect predictions of pain, and the inability to form an accurate model of future pain, can have clinical implications as well. A person repeatedly underestimating future pain experiences will be prone to dishabituation of pain responses. On the other hand, overprediction of pain can also have negative consequences. Although overprediction of pain does not produce disinhibition in itself, it can have a negative influ- 
ence on habituation by enhancing avoidance of pain. Avoidance of stimuli expected to be painful has been found to be prominent in chronic pain patients. If patients keep avoiding painful experiences, habituation will be prevented. Philips \& Jahanshahi (1985) have shown that whereas exposure to aversive auditive stimuli led to increased tolerance for these stimuli in migraine patients, avoidance led to decreased tolerance.

Considering the above, the absence of habituation of the experience of pain in chronic pain patients can be ascribed to different factors. First, chronic pain patients could have a fundamental deficit in habituation to painful experiences in general ${ }^{2}$. However, also more specific cognitive and behavioural factors could be responsible for absent habituation. Why the experience of pain is more prone to persist in some people than in others, is possibly related to differences in how people handle pain experiences. Speculatively speaking, a typical chronic pain patient could be a person paying excessive attention to pain and interpreting this pain in a catastrophic way, thereby enhancing the significance of the pain. If in the course of the pain condition the patient has not learnt to predict the painful consequences of various activities adequately, occasional underestimations of the pain experience can further hinder habituation. Patients who keep avoiding potential painful situations are also at risk of exacerbating their condition and promoting disability.

A fundamental deficit in habituation to pain stimuli would of course extend to all kinds of pain stimuli, clinical as well as experimental ones. But even when diminished pain habituation is due to more specific differences between cluronic pain patients and controls, this can influence the experimental pain situation. For instance, people prone to attend excessively to pain sensations and to attach exaggerated significance to these sensations, might generalize this behaviour to the laboratory situation. Avoidance of potentially painful situations could be generalized to the laboratory as well. So far, only few studies have tested whether differences in habituation to experimental pain stimuli can be found between chronic pain patients and controls. These studies will be reviewed in the next section.

\subsection{Chronic pain patients and differences in habituation to experi- mental pain stimuli}

There have been two studies which tested for differences between chronic headache patients and controls in the habituation of psychophysiological responses to aversive stimuli. In both cases, strong auditory stimuli were used, presumably aversive to patients with headaches. Ellertsen \& Hammerborg (1982) found that a series of twenty $95 \mathrm{~dB}$ auditory stimuli led to more pronounced heart rate responses and a slower habituation of these responses in migraine patients than in controls. Similarly, Passchier \& Orlebeke (1983) found a slower habituation of the skin conductance response (but not heart rate response) to 20 loud tones of $80 \mathrm{~dB}$ in migraine and

\footnotetext{
${ }^{3}$ This reasoning is at risk of being tautologicall: people who do not habituate to pain are said to have a fundamental habituation deficit, which in turn is "proved" by the fact that they do not habituate to pain.
} 
tension headache patients as compared to control subjects and agoraphobics. Another study used tones which were less intense $(70 \mathrm{~dB})$, and which might not have been experienced as aversive (Kroner, 1984). No differences were found between migraine patients, tension headache patients and controls on the habituation of frontalis $\mathrm{EMG}$, SCR, HR and respiration rate responses when 20 of these tones were presented. Differences between headache patients and controls in psychophysiological habituation perhaps only occur with aversive stimuli. Habituation of the subjective experience of pain has also been measured. An early indication for differences between chronic pain patients and controls in habituation of subjective pain responses comes from a study by Molin et al. (1973), which was actually not designed to measure habituation. This study determined pain threshold and pain tolerance in mandibular pain patients and in controls by means of three ascending series of electrical stimuli. Both pain threshold and pain tolerance showed an increase after repetition. However "for pain tolerance, this increase was considerably smaller in the patient group. There was no significant difference between the first and third test in mandibular pain patients, while there was in control subjects. Pain threshold showed a significant habituation in both groups.

A direct test for differences in habituation of pain responses between chronic pain patients and controls was performed in two succesive studies in our laboratory. The first study, by Brands \& Schmidt (1987) measured the habituation of a subjective pain response, while the second study (Antz et al., 1991) measured the habituation of both subjective and physiological responses to repeated pain stimuli. In the study of Brands \& Schmidt, eight successive trials of the cold pressor test were presented no CLBP patients and pain-free controls, and pain tolerance was measured. It was predicted that controls would show habituation, while CLBP patients would sensitize. However, the results indicated that in the control group, both habituating and sensitizing subjects were present. In the CLBP group, on the other hand, no learning effects were apparent. Thus, subjects neither habituated nor sensitized. In addition, CLBP patients had a lower pain tolerance than controls on the first test, accompanied by reports of more intense pain. Based on this finding, it was concluded: "It appears that the CLBP patients have already undergone a negative learning process, namely sensitization, which is responsible for their poorer endurance in CPT-1 (first cold pressor test) and which has left them with few, if any, opportunities to learn from repeated pain experiences."

The next experiment of Amtz et al. was also directed at CLBP patients. Twentytwo CLBP patients and twenty-one controls were given two series of 10 painful electrical shocks of constant intensity. Between the two series there was an interval of several minutes to assess whether dishabituation occured. The shock lewel was individually determined, so that all subject had an initial painfulness score of at least. $50 \mathrm{~mm}$ on a $100 \mathrm{~mm}$ VAS. The mean VAS score for this shock level was initially $63 \mathrm{~mm}$ on average in both groups. Physiological recondings of the phasic responses to every shock were made. Included were the variables: skin conductance response (SCR), heart rate response (HRR), finger pulse wolume response (FPVR), frontalis EMG response and paraspinal EMG response. The results showed that subjective VAS pain scores habituated to the same degree in both groups. By the end of the second trial, mean VAS score was 29.9 in the patients group and 30.0 in the control 
group. Also for the physiological variables, no differences in habination between the groups were found. SCR was the only physiological variable which clearly habituated, but the decrease was the same in both groups. HRR, FPVR, and both EMG responses did not show a clear habituation pattern in either group. The conclusion which can be drawn from this study is that CLBP patients are probably not character ized by a general impaiment of habituation to painful stimuli. The two variables that did show habituation (VAS and SCR) did so in both groups to an equal degree.

In sum, differences in habituation of responses to painful and aversive stimuli have been found in chronic pain patients, but this does not seem to be due to a fundamental deficit in habituation to pain stimuli in general. CLBP patients were shown to be capable of habituating to repeated electrical shocks. Perhaps impaired habituation is especially likely to occur when the repeated pain stimuli are significant to a patient, and when escape from or avoidance of these stimuli is possible. The anditory stimuli which were used in the studies described above seem to have been pain rellevant for headache patients. In the Brandls and Schmidt study, pain tolerance was measured. A pain tolerance test is basically a test of escaping from the pain situation. Thus, these studies differed on several points from the Arntz et al. study, in which pain stimuli very unlike clinical pain was used, and in which subjects had no opportunity to escape or avoid the next shock.

\subsection{Questions addressed in this dissertation}

The main question which is addressed with respect to habituation to pain stimuli in this dissertation is whether there is evidence that chronic pain patients do in fact show decreased habituation to experimental pain stimuli in certain circumstances. As has been reviewed above, the evidence so far has been limited and contradictory. The first two habituation studies presented in this dissertation used a mechanical pressure pain stimulus, which was reported to have a greater resemblance to clinical pain than electrical stimuli. Moreover, the stimulus is of longer duration than the short phasic electrical shocks. The habituation of various subjective and physiological parameters of pain is reported. Section 5.1 deals with subjective data only. Habituation of pain threshold, pain tolerance and of VAS pain ratings at intemediate stimulus intensity is measured in CLBP patients and controls after repeated pressure pain stimulation to the finger. Section 5.2 focusses on the habituation of psychophysiological responses to the pressure pain stimulus. Heart rate, respiration rate, skin conductance and electromyographic responses (frontalis and paraspinalis) were recorded. Finally, in section 5.9 data is reported on the habituation of electrical pain threshold and the spinal nociceptive flexion reflex.

The habituation studies in this dissertation only deal with the question whether CLBP patients do in fact show a different habituation pattern in certain circumstances. If such differences are found, the next step would be to elucidate under which particular conditions these differences are apparent. As has already been indicated, in some conditions CLBP patients can show habituation which is comparable to pain-free subjects. 



\section{THE EMPIRICAL STUDIES}

In the present chapter, nine experimental studies are presented which address the research questions formulated in the previous chapters. Although the theoretical discussion in these chapters has considered chronic pain in general, the empirical studies focus on CLBP patients specifically. Alll of the studies reported address one or more of the three major themes discussed in the previous chapter. These were:

1. Are there any differences in the psychophysiological activity and/or rasponsiveness between CLBP patients and controls?

2. Is there evidence for a different pain sensitivity in CLBP patients compared to control subjects, and can these differences be explained by the model of acute pain sensitivity which was formulated in section 3.8 ?

3. Are there differences between CLBP patients and controls in habituation to acute pain stimuli?

This chapter presents the studies in chronological order of performance rather than as centered around one of the three themes. The reason for this is that sometimes more than one theme is addressed by the same study. For instance, section 5.1 relates to differences in general pain sensitivity, as well as to differences in habituation of experimental pain responses between CLBP patients and controls. Section 5.2 relates to both the psychophysiological models of CLBP and to the habituation of pain responses.

This brief overview of studies outlines the rationale of the successive experiments performed for this dissertation and describes how the results of these studies have led to new research questions and further studies. The initial objective was to study habituation of pain responses in CLBP patients. The first experiment was set up, which measured habituation of both subjective and psychophysiologicall responses to pressure pain stimuli in CLBP patients and controls. The results of this experiment are presented in two separate papers (section 5.1. and section 5.2), the first reviewing the subjective data, the second the physiological data. In addition to habituation, the two other themes of this dissertation were included as secondary objectives of experiment 1. First, baseline differences in pain sensitivity between CLBP patients and controls were assessed at pain threshold and pain tolerance level. As far as I know, this study was the first to measure both variables in CLBP patients in the same experiment. The results of these measurements are reported in section 5.1. Next, in section 5.2, data pertaining to the hypothesis that CLBP patients show differences in baseline psychophysiological activity, or react to stress with larger psychophysiological responses, are reported. The reflex-spasm model, the stress-reaction model and the biomechanical model of CLBP are addressed.

The results of experiment 1 , in combination with the findings in the literature, led to the formulation of the model of acute pain sensitivity in chronic pain patients, described in section 3.8. The main objective of subsequent studies was now verifying the assumed relationship between stimulus intensity and pain sensitivity in CLBP 
patients, and to test the proposed mechanisms behind this. However, on several occasions the thernes of habituation and psychophysiological reactivity were again addressed. The aim of the second experiment was to confirm the relationship between experimental pain intensity and increased versus decreased pain sensitivity in CLBP patients. Further confirmation of this relationship was necessary because the differences found in the first experiment were non-significant. In experiment 2, pain threshold and pain tolerance for electrical shocks were measured, and in addition subjects also scored stimuli at different intensities in between pain threshold and pain tolerance. Also, a first attempt to test one of the mechanisms in the model (response tendencies) was made. The results of this experiment are reported in section 4.3 . Unfortunately, the experiment was unsuccessful, the finding of increased pain threshold and decreased pain tolerance in CLBP patients could not be replicated, and there was no indication for increased response tendencies in CIBP patients. However, in the discussion of section 5.3 it will be argued that the particular pain procedure which was used, and the operationalization of response tendencies, might be responsible for the negative findings.

The next two sections, section 5.4 and section 5.5 also report data from experiment 2. In section 5.4 the focus is once more on psychophysiological data. It is investigated whether there is concordance or discordance in CLBP patients and controls between skin conductance responses and the subjective pain scores to the electrical pain stimuli. Section 5.4 also includes data from an additional study in which SCR and subjective pain scores to repeated electrical shocks were measured. In section 5.5 the question of sensitivity for non-painful tactile stimuli in CLBP patients is addressed. This was measured by means of a two-point tactile discrimination test.

After the failure of the second experiment to find a difference in pain threshold and pain tolerance for CLBP patients, it was decided to establish first whether experimental counterimtation can decrease electrical pain sensation. A third experiment with normal control subjects was performed, and the influence of a pressure pain stimulus (the comterirritation stimulus) on the perception of electrical pain at threshold and at tolerance intensity was assessed. In addition, it was tested whether the recent finding that counterintitation can decrease pain discrimination could be confirmed. This sudy, reported in section 5.6, showed that the counterimitation stimulus was in fact able to decrease pain discrimination, but that it did not affect pain threshold and pain tolerance for electrical stimuli. Just as in the second experiment, it was noted that both pain threshold and pain tolerance for the electrical stimulus showed strong learning effects after repetition, perhaps overtuling the relatively small effects of counterirritation, Therefore, in the next experiments a stronger design had to be used.

Experiment 4 once more concerned CLBP patients. Again pain threshold and pain tolerance for electrical stimuli were measured, but the procedure was made more reliable by averaging the values obtained on several trials and by discarding the first measurement. By using a second pain stimulus (mechanical pressure pain), the design was further strengthened. In addition, this experiment measured pain discrimination to try to confim previous suggestions of decreased discriminative capacity in CLBP patients. A tone discrimination lest was also included to control for non-specific concentration/distraction effects. The results of this experiment are reported in section 
5.7. It will be seen that the data are not uniformly supportive of the hypothesis of increased pain threshold and decreased pain tolerance. Decreased pain sensitivity was found for most variables, that is, at threshold as well as at tolerance level. Although this might support the hypothesis that counterirritation can be triggered by chronic pain, such an interpretation does not conform to the previously hypothesized larger effect on low intensity pain levels. Moreover, it will be shown that pain discrimination was neither different for CLBP patients. This can be contrasted against the findings of the third experiment which showed that counterirritation can influence this variable. All in all, the model did not seem to be supported, and chronic pain possibly does not have a counterirritation effect.

The last experiment was set up as a more or less crucial experiment to establish whether CLBP does or does not activate DNIC. In this experiment, reported in section 5.9 , both pain threshold and the nociceptive flexion reflex of the biceps femoris were measured. Pain-free control subjects, subjects with acute post-operative pain, and chronic back pain patients were compared. DNIC does not only affect subjective pain indices, but also spinal nociceptive reflexes like the RIII reflex. Thus, it can be predicted that chronic pain patients have a higher RIII threshold. Moreover, acute pain should influence the two reflexes in the same way as chronic pain. In order to be able to make more reliable inferences as to whether DNIC is responsible for eventual differences between the groups, naloxone was administered. Naloxone has been shown to counter-act the DNIC effect, and baseline differences between the groups should disappear after the administration of naloxone. Before this final experiment, a pilot study was conducted, which is reported in section 5.8. This pilot study was intended to test our equipment, practice the procedure of eliciting reliable reflexes and replicate the finding that experimental counterirritation depresses the RIII reflex. The pilot study proved to be successful so that further research with chronic pain patients seemed to be worthwhile. So, the final experiment was performed. As a side issue of this experiment, one of the other themes of the disseratation was addressed once more. That is, it was investigated whether there is concordance and synchrony between pain perception threshold and RIII threshold in CLBP patients. In normal subjects, the two thresholds have been shown to agree very well. Results appear in section 5.9 . 


\subsection{CHRONIC LOW BACK PAIN AND THE REACTION TO REPEATED ACUTE PAIN STIMULATION ${ }^{1}$}

\section{SUMMARY}

Previous research has shown an interaction between chronic and acute pain behavior. Chronic low back pain (CLBP) patients seem to have a higher pain perception threshold and a lower pain tolerance than pain-free controls. The major focus is, however, on another aspect of pain behavior: habituation of the pain response after repeated stimulation. Habituation of the pain perception threshold was found for control subjects but not for CLBP patients. Inability to habituate to pain may be regarded as a risk factor in the dewelopment of CLBP.

\section{INTRODUCTION}

A distinction between acute and chronic pain is often made in literature (Sternbach, 1978). Pain is considered to be acute when it occurs as an automatic and immediate response to noxious stimulation. It is the consequence of some kind of tissue damage, and does not persist after this tissue pathology has recovered. Chronic benign pain,

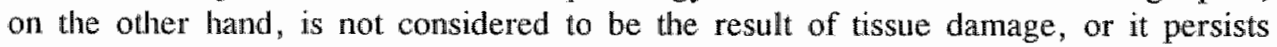
beyond the normal healing period for an acute injury or disease (Chapman \& Bonica, 1983). One of the major chronic benign pain syndromes is chronic low back pain (CLBP). A potentially fruiful question is how acute and chronic pain are linked in the CLBP syndrome.

Several studies have addressed this issue, and measured the response of CLBP patients (and other chronic pain patients as well) to acute pain stimuli. A distinction can be made between sudies which measured pain perception and those that measured pain tolerance or pain endurance. The studies of Yang et al. (1985), Naliboff et al. (1981) and Cohen et al. (1983) made use of a signal detection paradigm in which radiant heat stimuli were applied to CLBP patients and controls. These studies consistently found CLBP patients to thave a higher pain perception threshold.

When it comes to pain tolerance sudies a different picture emerges. Schmidt \& Brands (1986) and Brands \& Schmidt (1987) studied the pain tolerance of CLBP patients in the cold pressor test. Both studies showed CLBP patients to have a lower

\footnotetext{
TReprinted from: Peters, M.L. Schmidt. A.J.M. and van den Hout, M.A., Chronic low back pain and the reaction to repeated acute pain stimuli, Pain, 39 (1989) 69-76.
} 
tolerance of this stimulus, in combination with higher subjective pain reports.

Malow et al. (1980) also used a relatively long, gradually rising pain stimulus (Forgione-Barber pressure stimulator), with myofacial pain patients. Stimulation was stopped when subjects reported definite pain. Patients reached this point sooner than controls. Though Malow et al. had named this point "pain perception", it was not the first detection of pain. Viewed as a test of pain endurance, the results are in line with the Schmidt and Brands studies.

Another aspect of acute pain behavior is habiuation to the pain stimulus. So far, few studies have investigated the response of chronic pain patients to repeated experimental pain stimulation. It is, however, a theoretically important issue. Low back pain shows remarkable persistence; it can last for years. It looks as if CLBP patients do not habituate to their own pain. Do they perhaps have a fundamental inability to habituate to painful experiences?

Habituation to repeated acute pain stimulation has been shown to occur in healthy subjects. Strempel (1976), Glaser et al. (1959) and LeBlanc \& Potvin (1966) showed a decrease in subjective pain sensations after repeated immersions of a subject"s hand in coll water, and Condes-Lara et al. (1981) showed that pain intensity ratings decreased after repeated electrical tooth pulp stimulation. Pain perception threshold habituation after repeated electrical dental stimulation was shown by the studies of Mumford (1965) and Ernst et al. (1986). In addition, the latter study presented evidence of a greater impact of habituation on a low pain level (pain perception threshold) than on a more intense pain level (pain discomfort level, defined by the authors as "the intensity of stimulation that elicits a sensation which, if persisting, would prompt the subject to take aspirin").

Brands \& Schmidt (1987) studied the pain tolerance response of CLBP patients and control subjects to 8 successive cold pressor tests. They hypothesized that whereas controls would show increases in pain tolerance (habituation), patients would show decreases in pain tolerance (sensitization). However, they found no evidence of either habituation or sensitization in the patient group, while both processes were found in the control group. One subgroup of controls showed an increase in pain tolerance during the 8 trials, and another a decrease.

Arntz et al. (1991) found the habituation of pain intensity ratings on a visual analog scale, after repeating a moderately painful electrical shock 20 times, to be equal for CLBP patients and control subjects. Both showed habituation.

All in all, the data on habituation to pain stimuli in CLBP patients are somewhat inconsistent. However, this may be due to differences in dependent measures in the various studies.

In order to differentiate between habituation at different pain levels, the present study measured pain perception threshold, pain tolerance and pain intensity ratings at a level between perception threshold and tolerance.

The hypotheses tested in this study are: (1) CLBP patients have a higher pain perception threshold at the first presentation of the pain stimulus. (2) CLBP patients have a lower pain tolerance of the first pain stimulus. (3) In the control group there will be evidence of habituation of the pain response. In the patient group habituation will be absent. Habituation need not be the same at the different pain intensity levels. 


\section{METHOD}

\section{Subjects}

Forty male subjects participated in this study, 20 CLBP patients and 20 controls, matched for age. They were selected from a pool of subjects who had responded to an advertisement in a local newspaper. Some of them had been engaged in previous research. ${ }^{2}$

Inclusion criteria for patients consisted of having had pain in the back every day for at least 6 months, without a clearly defined organic cause. Exclusion criteria for patients and controls were cardiovascular disease and pain in, or damage to, hands and fingers at present or in the past.

The mean age of both patients and controls was 41 (range 29 - 56). The mean duration of back pain for patients was 11.5 years with a range of 2 to 30 years. All CLBP patients had a history of frequent medical consultations with a general practitioner or specialists. The most commonly prescribed medical treatment was physiotherapy, while 2 patients had been operated upon. None of the patients had participated in a pain programm or attended a pain clinic. Only 3 patients were currently under treatment for their back pain.

All subjects completed the entire session, and received a small financial reward after participation.

\section{Apparatus}

The pain stimuli were applied using a slightly modified Forgione-Barber pressure stimulator (Forgione \& Barber, 1971). A $1.7 \mathrm{~mm}$ wide plexiglass wedge with a pressure of 1700 grams was applied to the middle phalank of the second finger of the non-dominant hand. This finger was fixed in its place at the base of the apparatus, which was adaptable for fingers of different sizes.

The Forgione-Barber pressure stimulator elicits a gradually increasing pressure pain. It usually begins with a sensation of weight, which becomes painful after some time, and finally intolerable. The pressure stimulator makes it possible to measure pain perception wreshold and pain tolerance in the same trial. Moreover, it produces sensations which appear to be more closely related to clinical pain than those elicited

2 This previous research was considered not to interfere with the present study. It had taken place more then a vear before, and compared the physical performance of CLBP-patients and control subjects on a series of 6 working-to-tolerance treadmil tests. No experimental manipulations were introduced and no feedback was given in terms of "failure" or "succes". Thus, variables which could have affected future studies were absent.

In addition, there were no differences in familiarity of the present experimental setting between subjects who had participated in the prior study, and those who had not. The two studies were carried out in different locations and with different experimenters. The apparatus to which subjects were attached in the present study was new to all of them. 
by most other pain stimulator devices (Malow \& Olson, 1981).

\section{Procedure}

The experiment took place in a sound-proof laboratory with a constant temperature. The subjects were seated at a table with the pain stimulator in front of them. After a brief introduction the first pain perception threshold and pain tolerance measurements were made. Subjects were instructed to say "pain" when they first felt a painful sensation rather than just pressure, and "stop" when they did not want to tolerate the pain any longer. It was emphasized that the subject should try to endure the pain as long as possible. The time until the peception threshold and pain tolerance limit were reached was measured with a stopwatch. If the subject had not reported pain tolerance within three minutes after the onset of pain stimulation, the experimenter removed the pain stimulus in order to avoid possible damage to the finger.

After this initial assessment of pain sensitivity, 6 trials of identical pain stimulation followed. The level of pain stimulation was calculated on the basis of the first pain tolerance time. The time of the successive stimulus presentations was always $70 \%$ of the time of this first pain tolerance. With this procedure, an individually adapted stimulus intensity was obtained. This resulted in stimulation times which were never longer than 126 secs ( $70 \%$ of 3 minutes), while the shortest presentation time for one subject was $15 \mathrm{sec}$.

Subjects were informed that 6 stimulation trials would follow and that the stimulus would always be removed after a shorter time than the duration of the stimulus that they had tolerated the first trial. They were not told that stimulation time would be equal in each trial. Between the initial pain sensitivity assessment and the 6 succeeding presentations was an interval of about 15 minutes, in which electrodes for physiological measurements were attached, and during which baseline recording of physiological activity took place. Physiological data will be published elsewhere at a later date.

After this, 6 presentations of the pain stimulus at $70 \%$ of the tolerance time followed, with an interstimulus interval of 4 minutes. The subject was asked to indicate, at each stimulus presentation, when pain was first experienced (pain perception threshold). No further conversation was allowed during the stimulation period, except in order to stop the stimulus if, for any reason, it could no longer be tolerated. Two subjects did in fact stop stimulation one or more times before their individual time limit was reached.

After each of the 6 stimuli, subjects were presented a VAS scale on which they scored the maximum level of pain experienced during that stimulus. The extremes of the $10 \mathrm{~cm}$ scale were marked "no pain" and "unbearable pain". Before each stimulus, subjects rated on a similar VAS scale how much pain they expected on the following trial.

This stimulus presentation period took about 25-35 minutes (depending on the time limits set for a particular individual). After these 6 presentations there was another interval of approximately 10 minutes, during which the electrodes were removed. Next, one more pain stimulation took place. The procedure was identical to the initial 
pain sensitivity assessment; the subject was asked to indicate when the pain became intolerable, otherwise it was stopped after three minutes. Thus, another pain perception threshold and pain tolerance measurement was obtained. At this point the experimental session was terminated. The entire session took approximately 90 minutes.

\section{RESULTS}

\section{First stimulus presentation}

Pain perception thresholds were obtained for all subjects during the first stimulus presentation. However, three subjects sometimes failed to reach this threshold within the stimulus duration of the succeeding trials, and these subjects were therefore also left out of the analysis of the first trial. Thus, all analyses of pain perception thresholds are based on the same subjects. The pain tolerance data were complete for all subjects; analyses of this variable are therefore based on all subjects.

pain perc. thresih.

\begin{tabular}{llllllll}
\hline & $n$ & mean & sd & $n$ & mean & sd \\
controls & 18 & 27.3 & 19.9 & 20 & 137.8 & 58.8 \\
patients & 19 & 37.4 & 30.1 & & 20 & 117.3 & 61.1 \\
\hline
\end{tabular}

Table 5. 1: Mearr and standard deviation of pain perception threshold and pain tolerance in seconds for the first trial

Table 5.1 shows the pain perception threshold and pain tolerance for patients and controls. The results appear to follow the expected tendency; patients had a higher pain perception threshold and lower pain tolerance. Because this was in line with the predictions, 1 -tailed tests of significance were carried out ${ }^{3}$. The difference in pain perception threshold between patients and controls failed to reach significance $(\mathbb{F}=1.43, p=0.12)$. Because pain tolerance scores were not distributed normally, as the range of scores was limited at 180 seconds, a non-parametric test was carried out (Mann-Whitney mean test). Though the difference in tolerance scores did not reach

\footnotetext{
3 Tests directly derived from ane of the three hypotheses are 1-tailled tests. All other tests and post hoc tests are 2-tailled tests.
} 
significance, a trend in the expected direction was observed $(z=-1.35, \mathrm{p}=0.085)$.

\section{habituation}

Figure 5.1 shows the mean pain perception threshold for the 8 successive trials for patients and controls separately. It becomes clear from the figure that the threshold for controls increases from the first to the 8th trial, while it decreases for patients.

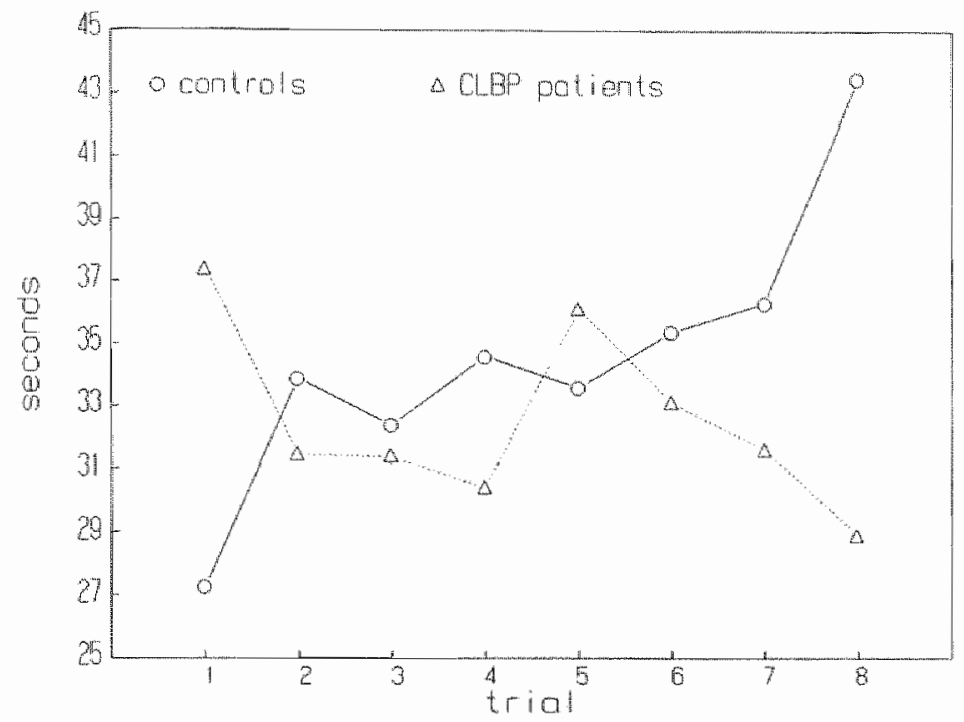

figure 5.1: Pain thresholds for 8 trials

Mean and standard deviations of pain perception thresholds for the 8 trials are presented in table 5.2. Multivariate analysis for differences in trends between the groups revealled a significantly different linear trend with 1 -tailed testing $(F(1,35)=2.81, p=0.050)$. Also, there was shown to be a significant difference in the cubic trend for CLBP patients and controls $\left(F(1,35)=5.93, p=0.020\right.$, two-sided). ${ }^{4}$

Inspection of the curves suggests that the major differences between controls and patients lie in the step from trial 1 to trial 2 and from trial 7 to trial 8 . While controls show evidence of habituation (an increase in pain perception threshold) between these trials, CLBP patients show sensitization (decrease in pain perception threshold). On the other hand, between trial 2 and 7 , there is a much less pronounced trend in both groups. When testing for differences in trend between groups for these trials only, thus leaving out trials 1 and 8 , the difference in trend is no longer significant.

"A cubic trend is not predicted directly from one of the hypotheses. Therefore it is a 2 tailled test. 
Thus, habituation of the pain perception threshold was found in the control group. It appears from figure 5.1 that the largest changes in pain perception threshold for controls took place between trials 1 and 2 and between trials 7 and 8. CLBP patients did not show habituation.

\begin{tabular}{ccccc} 
& \multicolumn{2}{c}{ controls $(n=18)$} & \multicolumn{2}{c}{ CLBP patient $(n=19)$} \\
\hline trial & mean & sd & mean & sd \\
\hline 1 & 27.3 & 19.8 & 37.4 & 30.1 \\
2 & 33.9 & 21.0 & 31.5 & 20.0 \\
3 & 32.4 & 23.9 & 31.4 & 24.7 \\
4 & 34.6 & 25.8 & 30.4 & 21.6 \\
5 & 33.6 & 29.1 & 36.1 & 33.1 \\
6 & 35.4 & 29.5 & 33.1 & 21.8 \\
7 & 36.3 & 32.7 & 31.6 & 20.4 \\
8 & 43.5 & 4.4 .9 & 28.9 & 21.9
\end{tabular}

Table 5.2: mean and standard deviation of pain threshold in seconds for patients and controls for 8 trials

Pain intensity ratings on the VAS were made during trial 2-7, after pain stimulation at a level between pain perception threshold and pain tolerance $(70 \%$ of the initial tolerance time). The results are presented in figure 5.2 and table 5.3 .

Multivariate testing for trends showed no differences between the two groups, nor was there a significant trend in the scores of either of the groups separately.

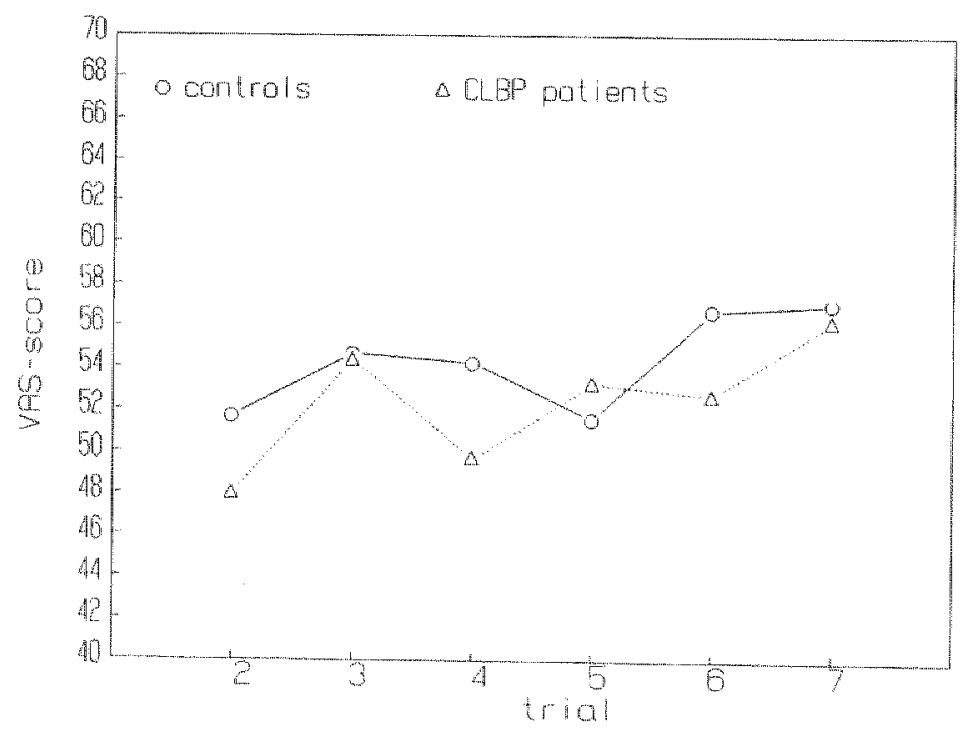

figure 5.2: VAS scores for 6 trials 


\begin{tabular}{lllll}
\hline irial & mean & sd & mean & so \\
\hline 2 & 51.7 & 22.4 & 48.0 & 20.1 \\
3 & 54.7 & 26.3 & 54.4 & 24.1 \\
4 & 54.3 & 27.8 & 49.7 & 26.8 \\
5 & 51.6 & 28.1 & 53.3 & 27.7 \\
6 & 56.8 & 27.2 & 52.7 & 27.0 \\
7 & 57.1 & 31.2 & 56.3 & 27.4 \\
\hline
\end{tabular}

Table 5.3: mean and standard deviation of VAS scores for patients and controls for 6 trials

Multivariate analysis of the expectation ratings showed that patients and controls did not differ significantly in their expectation of pain intensity in the next trial $(F(1,36)=1.77, p=0.139)$. Neither was there a difference in expectation between subjects who had tolerated the stimulus for the whole 180 seconds period and those who had not $(F(1,37)=0.58, p=0.74)$.

Pain tolerance was only measured twice: at trial 1 and at trial 8 . As figure 5.3 and table 5.4 show, pain tolerance increased in controls, from trial 1 to trial 8 , and decreased in CLBP patients.

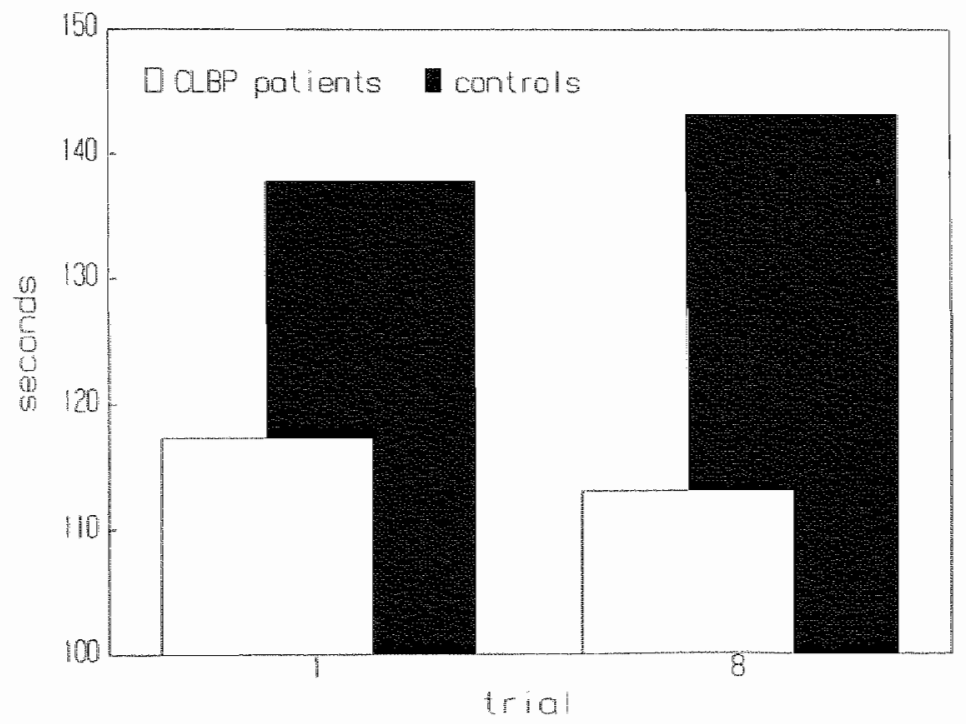

figure 5.3: Pain tolerance scores for the first and last trial 


\begin{tabular}{ccccc}
\hline trial & mean & mean & sd \\
\hline 1 & 137.8 & 58.5 & 117.3 & 61.1 \\
8 & 143.2 & 52.6 & 113.1 & 60.0 \\
\hline
\end{tabular}

Table 5.4: mean and standard deviation of pain tolerance scores for patients and controls for the first and last irial

Testing was carried out non-parametrically, because of the skewed distribution of the scores. The differences beteen trial 1 and trial 8 proved not to be significant for either patients or controls (Wilcoxon signed ranks test, $z=-0.97$ and $p=0.33$ for controls, $z=-0.43$ and $p=0.67$ for patients). The change in tolerance time was small compared to the possible range of scores. This might be due to the fact that 19 subjects reached the time limit of 180 seconds in the first trial. For them it was not possible to show habituation, and sensitization will also have been obscured. Another 4 subjects did not reach the 180 seconds limit at the first trial, but did so at the second. For these subjects, habituation has been deflated artificially. It might have been much larger. Thus, 23 out of 40 subjects show unreliable change scores, and it is obvious that this greatly invalidlates the results. By leaving out all those subjects who reached the tolerance time limit on either measurement, a different picture emerges for the control subjects. They show a statistically significant rise in tolerance time from 63.6 seconds to 108.4 seconds $(z=-2.37, p=0.018)$. The patients did not show a change in tolerance time from trial 1 to trial 8 (64.0 and 66.5 seconds respectively, $z=0.0, p=1.0$ ). However, these results are based on only 17 subjects ( 7 controls and 10 patients), who form, moreover, a specific subsample of subjects (the ones with the lowest tolerance times at the initial assessment).

\section{DISCUSSION}

The findings of a higher pain threshold and a lower pain tolerance for CLBP patients at first stimulus presentation showed a tendency in the expected direction, but the trends did not prove to be statistically significant. One of the reasons for this could have been the large interindividual variation in pain perception and tolerance scores.

Furthermore, 7 patients and 12 control subjects did not report reaching the limit of pain tolerance within the alloted period of 3 minutes, and were assigned the maximum score of 180 seconds. This has, however, artificially lowered the mean pain tolerance for both groups, and probably particularly so for controls, because more of 
tolerance for both groups, and probably particularly so for controls, because more of them reached the 3 minutes limit. A significant difference might have been obtained if the limit had been less restrictive.

The main interest of this study was, however, the habituation of pain sensitivity. It was shown that habituation of the pain perception threshold occurred only in controls. Patients did not show habituation.

For controls, the largest shifts in pain perception threshold took place from trial $\mathbb{1}$ to 2 and, from trial 7 to 8 . These trials seem to have contributed the most to the habituation effect of controls.

Between trial 1 and trial 2 and between trial 7 and trial 8 , there was an interval of 10-15 minutes, whereas between trials 2 and 7 the interstimulus interval was always 4 minutes. All stimuli were applied to the same spot on the same finger each time, and after 4 minutes, the impression of the preceding stimulus was usually still visible on the finger. Several subjects even indicated that they still felt their fingers to be a little tender when the new stimulus came. An interstimulus interval of 4 minutes seems to have been too short for complete recovery. A local physiological phenomenon, such as receptor sensitization, may have interacted with habituation in trials 2-7; whenever the interval was long enough for complete recovery (between trial 1 and 2 and trial 7 and 8) habituation was much stronger.

The pain intensity ratings (VAS scores) after $70 \%$ of stimulation in trials $2-7$ did not show habituation. No ratings were available for trials 1 and 8 . The fact that subjects might have been aware that every stimulus presentation was of the same duration does not seem to have influenced the VAS scores. By comparing the 6 expectancy ratings intraindividually, it became clear that subjects showed marked differences in expectation of the pain they would experience from trial to trial.

The lack of reliability of the pain tolerance assessment has already been pointed out. Because of the time limit, the change from the first to the second tolerance could not be calculated accurately. When all subjects for whom the change score could not be calculated had been left out of the analysis, the predicted result was found: controls increase their tolerance time, patients do not. However, as these results are based on such a small number of subjects, who in addition constitute a specific subgroup (subjects with low tolerance), they should be interpreted with some caution.

The main conclusion to be drawn from this study is that normal control subjects show habituation of the pain perception threshold after repeated pain stimulation, whereas patients do not. Whether this also applies to pain sensitivity at a higher level could not be demonstrated reliably, although there is a slight tendency in that direction for pain tolerance.

What does the finding of a lack of habituation add to our understanding of the CLBP syndrome? Do CLBP patients have a fundamental inability to habituate to painful experiences? If so, this should be considered a risk factor in the development of chronic pain.

Acute and chronic pain responses should not be considered to be unrelated. On the contrary, they seem to influence each other. If habituation to acute pain does not take place, the chance of the development of chronic pain behavior, shaped by operant 
conditioning (Fordyce, 1982), increases. Such chronic pain behavior can in turn influence the reaction to acute pain by stimulus generalization. Stimulus generalization is the process by which a particular response, which was at furst linked to only one specific stimulus (chronic pain), will be generalized to other stimuli which resemble the original one (acute pain) (Fordyce, 1983).

The evidence for an impairment of the habituational capacities of CLBP patients is as yet scarce. Future research should try to demonstrate whether a difference in pain perception habituation between CLBP patients and controls can be confirmed. A more reliable measurement of higher pain levels and tolerance would be welcomed. 


\title{
5.2 PSYCHOPHYSIOLOGICAL RESPONSES TO REPEATED ACUTE PAIN STIMULATION IN CHRONIC LOW BACK PAIN PATIENTS ${ }^{1}$
}

\begin{abstract}
Psychophysiological recordings (electrodermal activity, heart rate, respiration rate and frontalis and back muscle tension) were taken in chronic low back pain (CLBP) patients and control subjects during baseline conditions and during the presentation of six acute pressure pain stimuli. No baseline differences in back muscle tension between CLBP patients and controls were found, but CLBP patients did have higher baseline electrodermal activity. During pain stimulation, CLBP patients showed larger skin conductance reactions than controls. Allso, the CLBP patient group showed a marked increase in lumbar muscle tension during the test compared to baseline, whereas the EMG level of the control group remained unchanged. This increase in muscle tension seemed to be more related to the postural change than to the pain stimulus, and it is hypothesized that some CLBP patients react with exaggerated and non-functional back muscle contractions to slight changes in posture.

Contrary to expectation, no differences were found between CLBP patients and controls in physiological habituation after repeating the pain stimulus.

Comparison of subjective pain ratings and psychophysiological variables showed that for control subjects arousal and subjective pain were related. For CLBP patients there was no such clear relationship.
\end{abstract}

\section{INTRODUCTION}

People who suffer from chronic pain complaints tave been shown to respond differently to acute pain stimuli in the laboratory. Several studies have found a higher pain perception threshold Cohen et al., 1983; Naliboff et al., 1981; Yang et al., 1985) or a lower pain tolerance (Brands \& Schmidt, 1987; Schmidt \& Brands, 1986) for chronic pain patients.

In a previous article, we have shown that chronic low back pain (CLBBP) patients also differ from normal controls in another aspect of pain behavior, namely, the habituation to painful stimuli after repeated presentations. Whereas the pain perception threshold of

\footnotetext{
${ }^{1}$ Reprinted from: Peters, M.L. and Schmidt, A.J.M., Psychophysiological responses to repeated acute pain stimulation in chronic low back pain patients, Journal of Psychosomatic Research, 35 $(1991) 59-74$
} 
control subjects increased with successive trials, that of CLBP patients did not (Peters et al., 1989). The same has been found with pain tolerance measurements (Brands \& Schmidt, 1987). An inability to habituate to painful experiences has been implicated as a risk factor for CLBP (Peters et al., 1989). If CLBP patients have a fundamental deficit in habituating to painful stimuli, the habituation of physiological responses to painful stimulation which has been shown to occur in normal subjects (Badia \& Harley, 1970; Condes-Lara et al., 1981) could also be absent or diminished in these patients. Some earlier support for an absence of physiological habituation in chronic pain patients comes from the study of Ellertsen and Hammerborg with headache patients (Ellertsen \& Hammerborg, 1982). They demonstrated that repeated strong auditory stimulation (95 dB) led to more pronounced heart rate responses and a slower habituation of this response in migraine patients than in controls. Such a strong auditory stimulus is extremely aversive to migraine patients, perhaps even painful. Passchier \& Orlebeke (1983) found a slower habituation of the skin conductance response to a strong auditory stimulus in headache patients. On the other hand Kroner (1984), who measured several physiological variables (frontal and neck EMG, skin conductance responses, heart rate and respiration rate), did not find differences in habituation between chronic headache patients and controls after repetition of a $70 \mathrm{~dB}$ stimulus. It might be that differences only emerge when the stimulus is very aversive or painful.

Sternbach \& Tursky (1.965) have shown a relationship between pain attitude and habituation of physiological responses to acute pain stimuli. American women, who seemed to be more indifferent to pain than Irish, Italian and Jewish women, showed the fastest habituation of the palmar skin potential response to pain. If CLBP patients have a different attitude towards pain (e.g. lower pain tolerance), this could also be reflected in the habituation of their physiological pain response.

In addition, psychophysiological factors have been implicated in the etiology and the persistence of chronic low back pain. For instance, the reflex spasm theory of CLBP assumes that muscle contractions of the back muscles in CLBP patients occur, initiated as a protective response to some acute back injury (Collins et al., 1982; Dolce \& Raczynski, 1985; Nouwen \& Bush, 1984). However, after a while, when muscular tension is sustained, it can itself become the source of further pain. A vicious painspasm-pain cycle is established. Studies which have tried to verify this theory by measuring back muscle EMG in CLBP patients have shown diverse results (Dolce \& Raczynski, 1985; DeVries, 1968; Flor \& Turk, 1989; Hoyt et al., 1981; Jayasinghe et al, 1978).

An altemative model is the stress-reaction model of CLBP. This model assumes that there is an exaggerated response to mental stressors in CLBP patients, either specifically within the paraspinal muscles, or generally autonomic (Collins et al, 1982; Dolce \& Raczynski, 1985 ; Nouwen \& Bush, 1984). Studies testing this prediction have met with limited success (Nouwen \& Bush, 1984; Flor \& Turk, 1989; Cohen et al., 1986; Feuerstein at al., 1987). However, as Flor et al. (1985) pointed out, the stressors used in most studies were of little significance to the subject. They showed that when a stressior possessed personal relevance, the stress responses of CLBP patients were in fact stronger, but only the specific paraspinal muscle responses. These assumptions form the basis of the so called "diathesis stress model" which can be considered a specification 
of the general stress-reaction model.

Still another model, the biomechamical model of CLBP, argues that there might be differences in the patterns of muscular activity of back pain patients, which can eventually lead to pain by mechanical irritation of spinal structures (Dolce \& Raczynski. 1985). Several studies have found such differences in muscular activity (right-left differences, use of different muscle groups in performing a movement, and also lower muscle tension) during various movements and postures (Nouwen \& Bush, 1984; Ahern et a1., 1988; Cram \& Steger, 1983; Kravitz et al., 1981; Nouwen et al., 1987; Soderberg \& Barr, 1983).

This study will explore several aspects of psychophysiological responsiveness in CLBP patients. The first aspect to be considered is a baseline difference in back muscle tension between CLBP patients and controls, as hypothezised by the reflex spasm model. A second focus of this study is the way in which CLBP patients respond physiologically to an acute pain stimulus. Acute pain can be seen as a stressor which probably has relevance to pain patients. If the diathesis stress model is correct, a greater response to pain stimulation can be expected, but only in the paraspinal muscles. The general autonomic responses to a pain stimulus will not be different. A previous experiment using short duration electrical shocks as a pain stimulus indeed found only stronger muscular responses, especially with the paraspinal muscles (Arntz et al, 1991).

The third aspect which will be considered in this study is the habituation of the physiological pain response. Is the absence of subjective habituation paralleled by an absence of physiological habituation?

Finally, we will discuss the relationship between subjective pain perception and the psychophysiological responsiveness of the subjects.

\section{METHODS AND PROCEDURES}

\section{Subjects}

Twenty male CLBP patients and twenty controls, matched for age, participated in this study. The mean age for both groups was 41 years (range 29-56). The patients had been selected for the presence of low back pain for at least 6 months, for which no organic cause was suspected. Control subjects were free of pain. Both groups were sellected from a pool of subjects who had responded to an advertisement in a local newspaper. The subjects received a small financial reward after participation. Due to errors during recording, not all data are complete for all subjects.

\section{Apparatus}

pain stimulator

Pain stimuli were delivered by a slightly modified Forgione-Barber pressure stimulator 
(Forgione \& Barber. 1971). Mechanical pressure was exerted to the middle phalanx of the second finger of the non-dominant hand by means of a $1.7 \mathrm{~mm}$ plexiglass wedge, attached to a lever whit a weight. The total pressure applied to the finger was 1700 grams.

physiological measurements

The physiological measurements which were taken were skin conductance level (SCL), skin conductance resporse (SCR), frontalis $\mathrm{EMG}$, EMG at bilateral lumbar sites, heart rate (HR) and respiration rate (RR). A Beckman polygraph (type $\mathrm{R} 611$ ) was used for the recording of these variables. For all registrations Beckman $\mathrm{Ag}-\mathrm{AgCl}$ electrodes were used.

Skin conductance was reconded from the medial phalanxes of the second and first finger. Electrodes were filled with a self-made isotonic electrode creme (NaCL solution).

The electrodes for measuring paraspinal EMG were placed approximately $3 \mathrm{~cm}$ lateral to the spine in the lumbar region (level L3-LA), on both sides. A bipolar placement was used with an approximated vertical distance between the electrodes of $5 \mathrm{~cm}$. For EMG recording at the frontal site the electrodes were placed above the left brow.

Respiration rate was measured using a strain gauge attached to a strap placed around the subject's chest.

\section{Procedure}

Subjects had completed a trait anxiety questionnaire (STAI, trait version) and a depression scale (ZUNG) at home. In addition, patients had also completed a questionnaire in which they were asked some general questions about their back pain and medical history. At the beginning of the experiment the subjects completed another questionnaire, the state anxiety version of the STAI. Patients also rated their current back pain on a visual analogue scale (VAS). Subsequently, pain perception threshold and pain tolerance for the pressure pain test were measured (in seconds, with a maximum of 180 seconds). After this, the electrodes for physiological recordings were attached to the subject. After checking the signals and allowing some time for stabilization, a two nwwte baseline reconding was made. The position of the subject was sitting at a table in an erect chair with the back supported, the hands placed on the table. The subject was asked to sit still and relax as much as possible.

After the baseline recording six pain stimuli were applied, always lasting $70 \%$ of the first measured pain tolerance time. All stimuli were applied to the medial phalanx of the second finger of the non-dominant hand. The inter-stimulus interval was 4 minutes. Subjects had been instructed to keep their finger in the device from 30 seconds before stimulation began (prestimulus period) until 30 seconds after stimulation (recovery period). Meanwhile, physiological recording took place. In this way, movement during recording was prevented.

This procedure resulted in 19 periods of physiological recordings for each subject: a baseline recording of 2 minutes, six prestimulus recordings of 30 seconds, six stimulus recordings varying in length between individuals and 6 recovery recordings of 30 seconds. 
Subjective data (pain threshold and VAS ratings) were also collected for each stimulus. These data were reported in a previous article (Peters et al., 1989). The entire session required approximately 90 minutes.

\section{Data reduction and analysis}

\section{Skin conductance wariables}

For all periods, the lowest SCL reading during that period was assessed. The number of skin conductance fluctuations during the same periods was counted. A response was defined as an increase of skin conductance of at least 0.05 micromho. Maximal SCR was defined as the largest SCR during the entire stimulation period.

\section{Heart rate variables}

The ECG signal was analyzed by off-line computerized detection of R-tops, thus presenting interbeat interval (IBD) times, at an accuracy of 1 millisecond. These IBI's were later converted to $\mathrm{HR} / \mathrm{min}$ for every second, by weighing every beat in a particular second by the proportion of the second it occupied. Several ways for further analysis of the signal were explored. The most straightforward method was to calculate average HR for the different periods within the experiment. Also, within each stimulation period the mean HR before pain was reported, and the mean HR after pain was reported, was calculated.

To see whether a phasic response was present, a second by second analysis was performed on the first 10 seconds of the first stimulation period. The same was done for the 10 seconds after pain was reported. In addition, a trend analysis was performed to see whether there was an ongoing acceleration within the stimulation period.

Finally, variability in $H R$ was assessed, using the variation coefficient (VARCOEF) as an index. The VARCOEF computes the standard deviation of all HR scores within a period and corrects this for mean $H R$, on which it has been shown to be dependent (VARCOEF $=$ Stdev/mean HR) (Dellen et al., 1985).

\section{Respiration variables}

Off line, the computer provided the times of maximal inspiration and expiration. Average respiration cycle time was calculated for complete periods.

EMG variables

The signal was filtered, and only frequencies between 20 and $100 \mathrm{~Hz}$ were passed. Hereafter, off-line sampling took place by a PDP 11 computer, with a frequency of 500 Hz. Because sampling and $A-D$ converting proved to be very time-consuming, the analysis was limited to the first trial only. Five 10-second samples were taken for every EMG record : the last $10 \mathrm{sec}$. of baseline, the last $10 \mathrm{sec}$. of the prestimulus period, the first $10 \mathrm{sec}$. of the stimulus period, the last $10 \mathrm{sec}$. of the stimulus period and the first $10 \mathrm{sec}$. of the recovery period. Additional programmature provided an averaged level of muscle tension in microvolts per sample.

\section{Sratistical analyses}

Mean baseline values for controls and CLBP patients were compared using t-tests. One-tailed tests were used whenever predicted effects were tested. To test for differences in responsivity to the pain stimulus between the groups, MANOVA's were performed 
according to the method of O'Brian \& Kaiser' (1985). The main group effect compares overall differences in activity between patients and controls. The period effect contrasts the prestimulus period and the recovery period with the stimulus period and indicates whether a particular variable responds to pain stimulation. The period $\mathrm{x}$ group interaction tests for differences between patient and controls in the pain response.

Habituation was analyzed by means of MANOVA polynomial trend analyses. Only the linear trends are considered. The trial main effect indicates a decrease (or increase) after repeated trials of overall activity during all periods, and the trial $x$ group interaction tests for differences between patients and controls in this change. The trial $\mathrm{x}$ period interaction indicates the actual habituation effect: it tests whether the pain response decreases after repeated trials. Differences in habituation between CLBP patients and controls are indicated by the trial $x$ period $x$ group effect.

To see whether the subjective pain judgements were related to the physiological measurements, correlations between these variables were computed. Between subject analysis consisted of correlating the mean pain rating for all six trials with the mean physiological activity during these trials. It indicates whether the subjects with the highest pain ratings are also the physiologically most active subjects. The relationship between subjective and physiological variables within subjects was assessed by rank ordering the trials according to rated painfulness and to compare this to the (linear increasing) trends. in the physiological data.

\section{RESULTS}

\section{Descriptive analysis of the patient group}

Analysis of the back pain questionnaire indicated that the duration of back pain in this patient group ranged from 2 to 30 years, with a mean duration of 11.5 years.

The ratings on a VAS given by patients in the laboratory of their current back pain ranged from 5 to 69 , showing large individual variation in back pain intensity with which patients entered the experiment.

\section{Baseline differences between patients and controls}

\section{Psychological measurements}

Because it has been shown before that CLBP patients score consistently worse on all kinds of psychological variables than controls (Feuerstein et al., 1987), it was predicted that patients would prove to be more anxious and depressed. As can be seen from table 5.5 , trait anxiety and depression were significantly higher in CLBP patients, whereas state anxiety showed a trend towards this.

Skin conductance wariables

Table 5.5 shows that baseline SCL was significantly higher for CLBP patients than 
for controls. There was also a trend towards more spontaneous fuctuations for CLBP patients during this period.

\begin{tabular}{|c|c|c|c|c|c|c|c|}
\hline & $\mathrm{n}$ & $\begin{array}{l}\text { CONT } \\
\text { mean }\end{array}$ & $\begin{array}{l}\text { TROLS } \\
\text { (sd) }\end{array}$ & $n$ & $\begin{array}{l}\text { CLBP } \\
\text { mean (sd) }\end{array}$ & $\mathrm{t}$ & $\mathrm{p}$ \\
\hline \multicolumn{8}{|c|}{ psychologicall variables } \\
\hline depression & 19 & 33.4 & $(7.92)$ & 20 & $40.0(10.91)$ & 2.40 & 0.011 \\
\hline trait anxiety & 19 & 35.1 & $(8.02)$ & 20 & $41.9(12.82)$ & 1.96 & 0.028 \\
\hline state anxiety & 20 & 32.4 & $(5.52)$ & 20 & $36.0 \quad(7.87)$ & 1.67 & 0.056 \\
\hline \multicolumn{8}{|c|}{ skin conductance variables } \\
\hline $\mathrm{SCL}$ & 17 & 6.77 & $(3.54)$ & 19 & $9.52 \quad(4.69)$ & 1.97 & 0.029 \\
\hline number of fluc. & 17 & 4.47 & $(4.39)$ & 19 & $7.16 \quad(5.59)$ & 1.59 & 0.061 \\
\hline $\begin{array}{l}\text { EMG variables } \\
\text { left lumbar }\end{array}$ & 19 & 3.67 & $(1.90)$ & 20 & $3.19 \quad(4.01)$ & 0.82 & 0.270 \\
\hline right lumbar & 20 & 3.48 & $(1.83)$ & 19 & $3.10 \quad(0.85)$ & 0.46 & 0.325 \\
\hline frontal & 20 & 3.23 & $(0.98)$ & 20 & $2.88 \quad(0.82)$ & 1.23 & $\begin{array}{l}0.450 \\
{[\mathrm{tt})}\end{array}$ \\
\hline heartrate & 18 & 73.9 & $(10.9)$ & 18 & $73.4 \quad(10.7)$ & 0.13 & 0.895 \\
\hline resp. rate & 20 & 14.7 & $(3.4)$ & 20 & $15.1 \quad(3.3)$ & 0.40 & $\begin{array}{l}0.689 \\
(t t)\end{array}$ \\
\hline
\end{tabular}

Table 5.5: Baseline values for different variables for control subjects and CLBP patients. Testing was performed by means of t-tests. All reported p-values are one tialed, except for the last three variables in which 2-tailed test were used (tt). For these variables no prediction was made of a difference between the groups in either direction.

\section{EMG variables}

There were no difference in back musclie tension during baseline conditions between patients and controls. For the frontalis muscle tension, again, no difference was found (table 5.5).

Heart rate and respiration variables

There were no baseline differences between patients and controls in heart rate and respiration rate (table 5.5 ).

To summarize, CLBP patients were more anxious and depressed than controls, and they had higher electrodermall activity during baseline measurement. No other differences in psychophysiological variables were found between the groups during baseline. 
Differences in psychophysiological responses to pain stimuli

The results from all analyses are displayed in table 5.6. Reported p-values are onetailed.

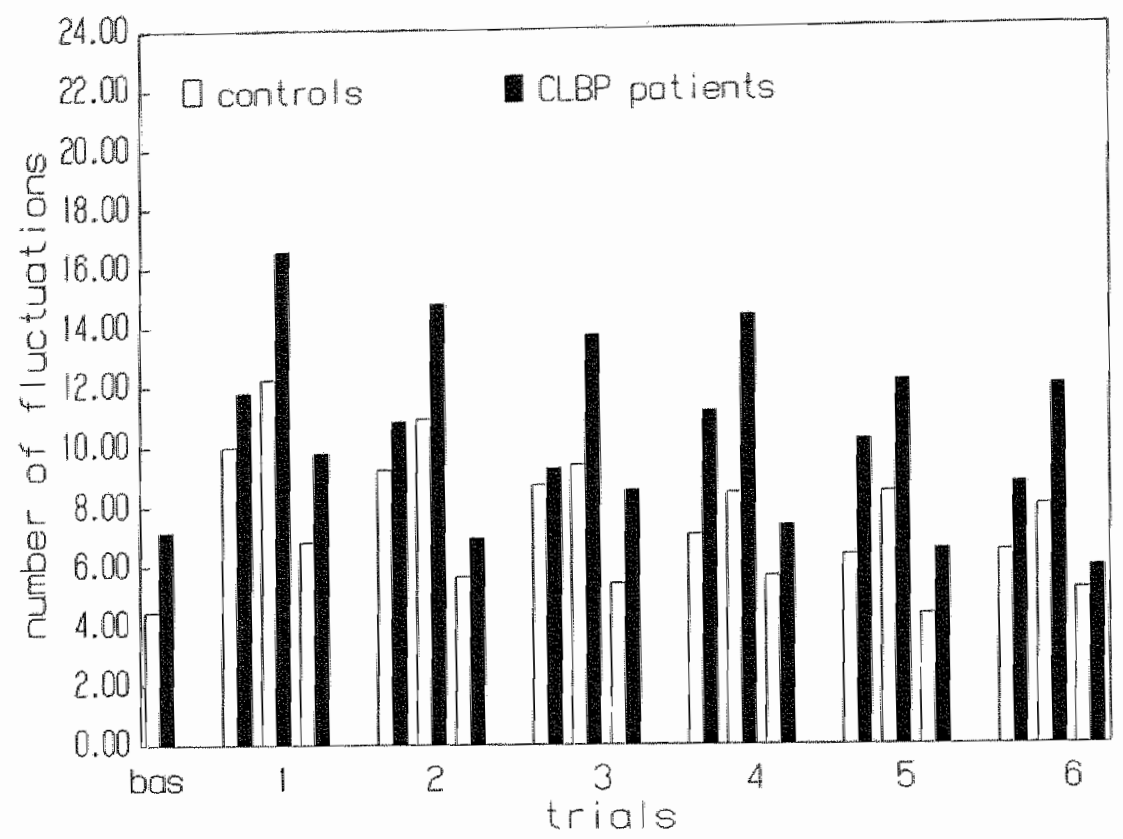

figure 5.4: Mean number of skin conductance fluctuations per minute during the baseline and three different periods within six trials. The left bar within one trial represents the prestimulus period, the bar in the middle represents the period when the pain stimulus is given and the right bar represents the recovery period. CLBP patients showed more skin conductance fluctuations during the basaline period, as well as during all other periods. In addition, the increase in the number of fluctuations in the stimulus period is greater for patients.

\section{Skin conductance variables}

The group effect for SCL almost reached significance. There was a trend towards higher SCL.'s for patients during all measurement periods. There was no significant period effect

or period $\mathrm{x}$ group interaction. As shown by the significant group effect, patients had a higher number of mean fluctuations in each period. The period effect also proved to be significant, which shows that the number of SC fluctuations increase during stimulation (figure 5.4). In addition, there was a significant group $x$ period effect indicating that CLBP patients showed a greater increase in number of fluctuations during the pain stimulus.

Figure 5.5 shows the maximal SCR during the stimulus period for patients and 
controls. It is clear from the figure that patients showed greater maximal SCR's than controls. The group effect was statistically significant.

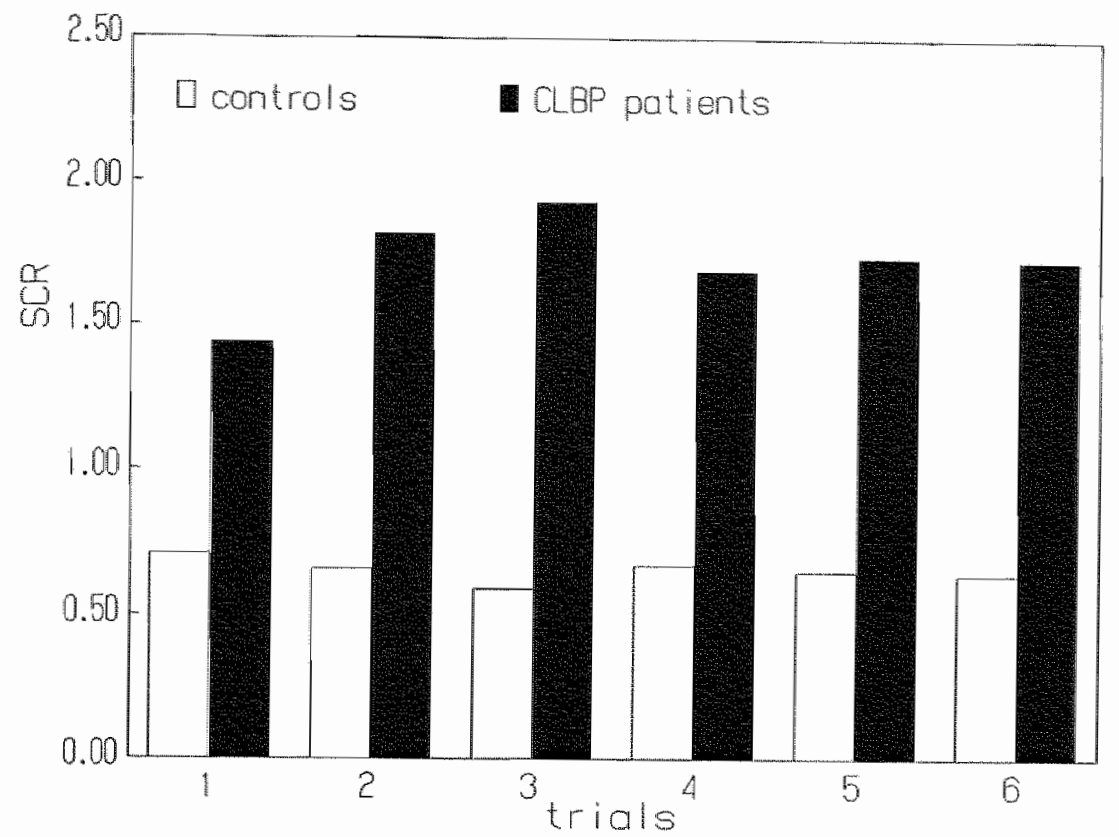

figure 5.5: Mean maximal skin conductance responses in (micromho) during the six presentations of the pain stimulus. CLBP patients had larger maximal SCR's during all trials.

\section{Heart rate variables}

Second by second analysis of the first trial did not reveal a conistent phasic response at the beginning of stimulation or after the report of pain. Further analysis of the second by second data for the whole stimulation period did not indicate the presence of systematic responses at any other time during this period. Finally, no consistent trends were present during the stimulation period.

Considering the foregoing, only mean HR and HR variability for complete periods were anallyzed further. For mean HR, the period effect was significant. Post hoc analysis showed that this was due to a decrease in HR from stimulus to recovery period $(F(1,34)=5.70, p=0.023$, two-tailed). Contrary to expectation no increase in HR took place from prestimulus to stimulus period. There was no main group effect or a group $x$ period interaction. In addition, mean HR before and after pain was reported did not differ (mean HR before pain threshold: 72.6, after pain threshold: 72.2, CLBP patients and controls together).

For HR variability also, only a period effect was found. HR variability decreased during stimulation. No group effects were found. 


\section{Respiration variables}

For RR there were no significant multivariate effects. However, the univariate period effect indicated a significant increase in respiration rate from prestimulus period to stimulus period $(F(1,38)=4.20, p=0.047$, two-tailed $)$.

\begin{tabular}{|c|c|c|c|}
\hline EFFECT & $d f$ & $F$ & $p$ \\
\hline \multicolumn{4}{|l|}{$\mathrm{SCL}$} \\
\hline group & 1.34 & 2.83 & 0.051 \\
\hline period & 2,33 & 1.47 & 0.123 \\
\hline group $x$ period & 2,33 & 1.02 & 0.186 \\
\hline number of SCR's & 1,34 & 4.14 & 0.025 \\
\hline period & 2,33 & 30.7 & 0.001 \\
\hline group $x$ pariod & 2,33 & 2.58 & 0.046 \\
\hline \multicolumn{4}{|l|}{$\max . \mathrm{SCR}$} \\
\hline group & 1,34 & 11.20 & 0.002 \\
\hline \multicolumn{4}{|l|}{ mean $\mathrm{HR}$} \\
\hline group & 1,34 & 0.06 & 0.410 \\
\hline period & 2,33 & 3.11 & 0.029 \\
\hline group $X$ period & 2.33 & 1.50 & 0.220 \\
\hline \multicolumn{4}{|l|}{ HR variability } \\
\hline group & 1,29 & 0.04 & 0.423 \\
\hline period & 2,28 & 8.81 & 0.001 \\
\hline group $x$ period & 2,28 & 0.39 & 0.339 \\
\hline \multicolumn{4}{|l|}{ resp. rate } \\
\hline group & 1,38 & 0.02 & 0.446 \\
\hline period & 2,37 & 2.06 & 0.071 \\
\hline group $\times$ period & 2,37 & 0.57 & 0.286 \\
\hline \multicolumn{4}{|l|}{ EMG left lumbar } \\
\hline group & 1.36 & 2.02 & 0.082 \\
\hline period & 4,33 & 1.51 & 0.111 \\
\hline group $\times$ period & 4,33 & 2.96 & 0.017 \\
\hline \multicolumn{4}{|l|}{ EMG right lumbar } \\
\hline group & 1,35 & 0.04 & 0.4119 \\
\hline period & 4,32 & 1.62 & 0.097 \\
\hline group $x$ period & 4,32 & 1.56 & 0.105 \\
\hline \multicolumn{4}{|l|}{ EMG frontel is } \\
\hline group & 1,37 & 1.27 & 0.133 \\
\hline period & 4,34 & 0.96 & 0.222 \\
\hline growp x period & 4,34 & 11.44 & 0.121 \\
\hline
\end{tabular}

Table 5. 6: Results of Manova tests for differences between groups and periods. The group effects indicate an overall difference between CLBP patients and controls on a variable. The period effect tests whether that particular variable responds to the pain stimulation. Finally, the group $x$ period inter-action denotes whether the response of a variable differs between the groups. P-values are one tailed.

\section{EMG variables}

Figure 5.6 shows the data of the three EMG recordings. For the left lumbar recording 
it appears that CLBP patients had a higher level of muscle tension during all measurement periods except baseline. This is also shown by the significant group $x$ period effect. Post hoc analysis revealed a significant increase in muscle tension for CLBP patients from baseline to prestimulus period, which remained stable during the rest of the recording period. Controls did not show a significant change in muscle tension.

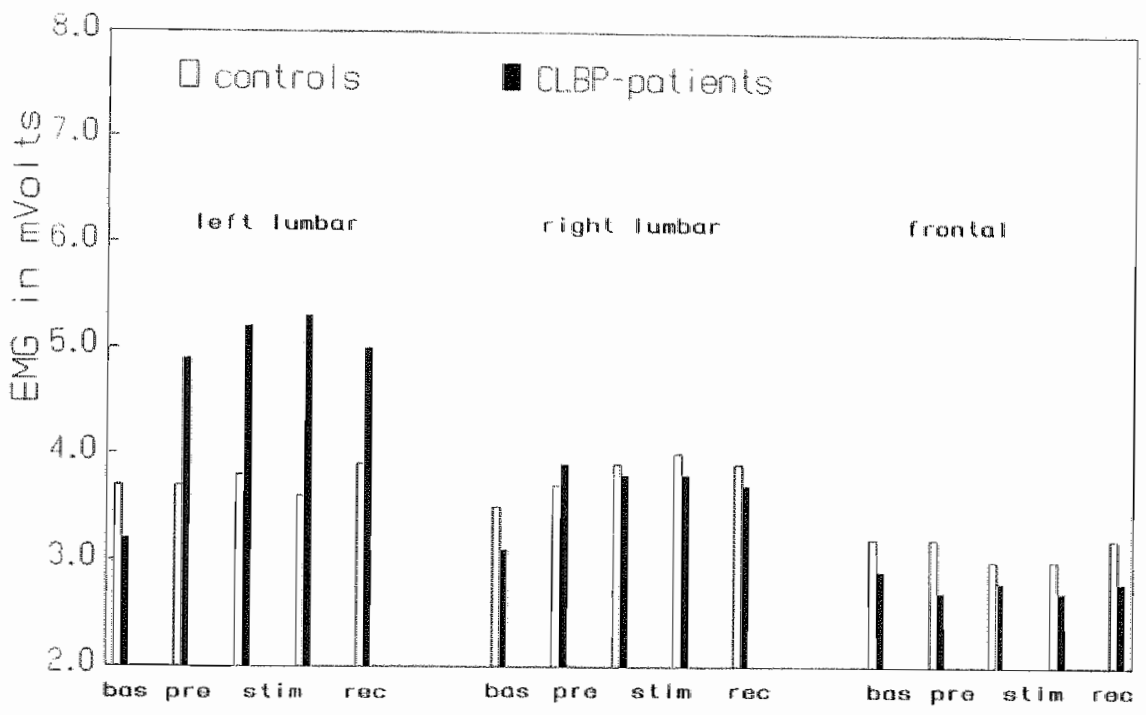

figure 5.6: Mean muscle tension (in $\mathrm{mV}$ ) for the three recording sites during the last $10 \mathrm{~s}$ of the baseline and four 10-s periods within the first trial (10s just befare stimulation. the first $10 \mathrm{~s}$ of stimulation, the last $10 \mathrm{~s}$ of stimulation and 10 s just after stimulationl. CLBP patients only reacted differently with the left lumbar muscles.

The same pattern was seen for the right lumbar EMG record, though less pronounced. CLBP patients had a slightly lower muscle tension during the baseline period, which increased to values comparable to the control group during the prestimulus period. Controls had stable EMG values. The period $x$ group interaction did not reach statistical significance.

Inspection of the raw EMG traces showed that the difference between the left and right recording was due to a greater number of patients who reacted on the lleft side exclusively, compared to those who reacted on the right side exclusively. The reactions themselves were on the average of equal magnitude on both sides.

Some typical reactions of four of the patients are shown in figure 5.7. As can be seen, muscle tension immediately increased as the finger was placed in the device and stayed at this level during the prestimulus period, the stimulus period, and the recovery period. 
Thus, the pain stimulus did not further increase the tension. When the subject removed his finger from the stimulation device, tension was immediately reduced to baseline values.

Frontalis EMG did not differentiate between patients and controls. Most subjects did not react to the pain stimulus with changes in frontalis muscle tension.

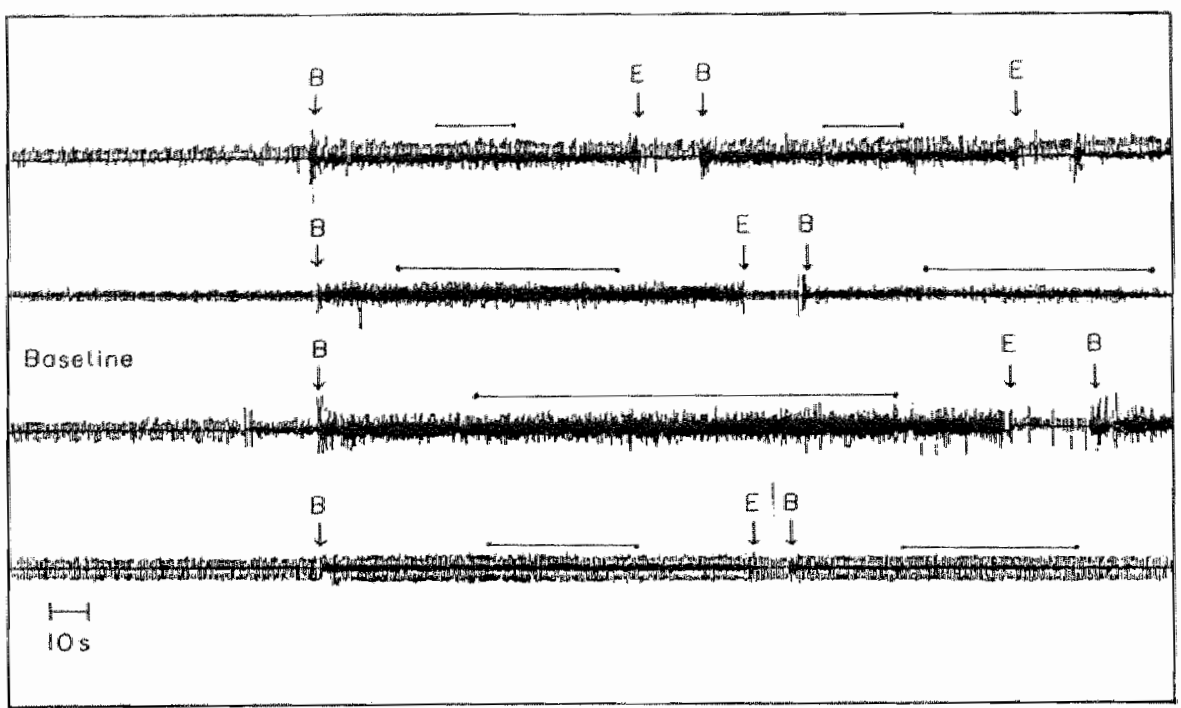

figure 5.7: Four examples of the lumbar EMG reaction on the left side. The left of the figure shows a part of the two-minute baselline recordings. The arrow marded ' $B$ ' indicates the beginning of a triall, when the finger is placed in the device. Sometimes a slight movement artefact is observed. The arrow marked ' $E$ ' indicates the end of a trial, when the finger is removed from the device. The lines above the traces indicate the presence of the pain stimulus on the finger. As this illustration clearly shows, increases in muscle tension coincides with the time the finger is in the device, and not with the presence of the pain stimulus.

To conclude, both the overall skin conductance activity and the skin conductance responses were higher for CLBP patients. The left lumbar EMG of CLBP patients also showed more reactivity compared to controls. For right lumbar EMG this was less pronounced. Contrary to our expectations, there were no increases in mean HR during pain stimulation. However, HR decreased from stimulus to recovery period. Also, the variability of HR decreased during stimulation, while RR increased. The HR and RR responses did not differentiate between CLBP patients and controls. For frontalis EMG, no response to pain stimulation could be detected. 


\section{Differences in habituation of psychophysiological responses}

The results of all trend analyses are displayed in table 5.7. Reported p-values are twotailed.

\begin{tabular}{|c|c|c|c|}
\hline$\overline{E F F E C T}$ & df & $F$ & $p$ \\
\hline \multicolumn{4}{|l|}{ number of SCR's } \\
\hline trial & 1,34 & 34.34 & 0.001 \\
\hline trial $\times$ group & 1,34 & 0.01 & 0.920 \\
\hline trial $x$ period & 1,34 & 6.02 & 0.020 \\
\hline trial $x$ group $\times$ per. & 1,34 & 0.05 & 0.824 \\
\hline \multicolumn{4}{|l|}{$\max$ SCR } \\
\hline trial & 1,34 & 0.10 & 0.759 \\
\hline \multicolumn{3}{|l|}{ mean $H R$} & 0.540 \\
\hline trial & 1,34 & 13.38 & 0.001 \\
\hline trial $\times$ group & 1,34 & 3.34 & 0.076 \\
\hline trial $\times$ periad & 1,34 & 1.69 & 0.203 \\
\hline trial $x$ group $x$ periode & 1,34 & 1.19 & 0.283 \\
\hline \multicolumn{4}{|l|}{ HR variability } \\
\hline trial & 1,29 & 1.41 & 0.244 \\
\hline trial $x$ group & 1,29 & 4.69 & 0.039 \\
\hline trial $x$ period & 1.29 & 0.07 & 0.792 \\
\hline trial $\times$ group $\times$ periode & 1,29 & 1.10 & 0.303 \\
\hline \multicolumn{4}{|l|}{ respiratrion rate } \\
\hline trial & 1,38 & 8.67 & 0.005 \\
\hline trial $\times$ group & 1,38 & 2.82 & 0.101 \\
\hline trial $x$ period & 1,38 & 1.37 & 0.249 \\
\hline trial $\times$ group $\times$ periode & 1,38 & 2.70 & 0.107 \\
\hline \multicolumn{4}{|l|}{ EMG } \\
\hline trial & 1,17 & 1.11 & 0.306 \\
\hline trial $\times$ group & 1,17 & 1.18 & 0.290 \\
\hline
\end{tabular}

Tabie 5.7: Results of the MANOVA polynomial trend analysis testing for habituation effects. The triall main effect indicates an overall increase or decrease of activity of a variable. The trial $x$ group effect tests for differences between the two groups on this trend. The trial $x$ period effects tests whether there is a habituation of the specific pain resiponse, while the trial $x$ group $x$ period interaction tests whether this habituation differs between CLBP patients and controls. Reported p-values are two-tailled.

\section{Skin conductance variables}

For skin conductance fluctuations, there was a significant trial and trial $\mathrm{x}$ period effect, showing that the total number of fluctuations in all periods decreased after repeated trials and also that the increase during the stimulus period became less. Thus, habituation was present. There were no group effects. The largest skin conductance response during the stimulation period did not decrease after repeated trials (trial effect). Nor was there a group $x$ trial effect. 


\section{Heart rate wariables}

The trial main effect for HR was statistically significant. There was a decrease in owerall HR during the course of the experiment This decrease appeared mainly in the control group, though the trial $x$ group interaction failed to reach significance.

The trial $x$ period effect was not significant. The observed HR decrease in the recovery period was also subjected to a trend analysis. No habituation of this response was found $(\mathrm{F}(1,34)=1.19, \mathrm{p}=0.283)$.

The wariability coefficient (VARCOEF) did not show a main trial effect for the linear trend. However, there was a significant trial $x$ group interaction. Whereas CLBP patients increased their overall variability slightly in the course of the experiment, controls did not. The other interaction effects were not significant.

\section{Respiration variables}

Mean RR decreased slighly after repeated trials. However, there was no trial $x$ group interaction. The univariate period effect observed above did not show habituation.

\section{EMG variables}

Analysis of the first trial had already shown that the frontal muscle did not react to pain stimulation, and later trials were only inspected visually. There appeared to be neither an increase nor a decrease in overall frontal muscle activity in the course of the experiment.

The lumbar EMG data from subjects who did not react at all, or only once on one of the later trials, were also only visually inspected. No overall increases or decreases in muscle tension could be detected. To test whether there was a systematic increasing or decreasing trend in the magnitude of the response for the subjects who did show a response $(n=19)$, one sample per trial (from the stimulation period) was collected for further analysis. It was shown that there was no significant trial effect nor a significant trial $x$ group interaction. Thus, the EMG response, when present, did not habituate.

In summary, the variables spontaneous fluctuations, HR and RR showed a significant trial main effect, that is, an overall decrease after repeated trials. This could indicate a general reduction of arousal during the course of the experiment. Only HR changes differentiated between CLBP patients and controls: the patient group did not display the overall decrease in HR. At the same time, CLBP patients showed an increase in HR variability after repeated trials. The trial $x$ period analyses showed that the only response which habiuated was the increase in number of fluctuations during stimulation. There was no group effect on this trend, which indicates that CLBP patients habituated at the same rate as controls. There was no evidence of habituation for any of the other responses.

\section{Comparison between subjective and psychophysiological responses}

\section{between subjects}

For the control group, the correlations between the VAS ratings of subjective painfulness of a stimulus and the 3 skin conductance variables during stimulation were significant (table 5.8). The highest correlation apperared to be with the number of 
fluctuations. Subjective pain was not only related to electrodermal activity during stimulation, but also with baseline activity. Again, the number of fluctuations correlated most highly with the VAS scores.

CLBP patients did not display such consistent significant correlations between skin conductance variables and subjective pain ratings. Only with mean number of fluctuations during stimulation there was a significant correaltion, although lower than for the control group. For both groups, no correlation between mean HR and RR during stimulation or during baseline and subjective pain was present.

Within subjects

Within subjects, there was no relationship between the VAS ratings and any of the physiological variables, neither for patients nor controls.

\begin{tabular}{lll}
\hline & $\begin{array}{l}\text { Subjective } \\
\text { pain ratings } \\
\text { Controls }\end{array}$ & $\begin{array}{l}\text { Subjective } \\
\text { pain ratings } \\
\text { CLBP patients }\end{array}$ \\
\hline Mean SCL during 6 trials & $\begin{array}{ll}r=0.46 \\
n=17\end{array}$ & $\begin{array}{l}r=0.15 \\
n=19\end{array}$ \\
& $p=0.032$ & $p=0.264$ \\
Mean SCR during 6 trials & $r=0.41$ & $r=0.14$ \\
& $n=19$ & $n=19$ \\
& $p=0.040$ & $p=0.178$ \\
Mean number of & $r=0.61$ & $r=0.45$ \\
fluctuations during 6 trials & $n=17$ & $n=19$ \\
Baseline SCL & $p=0.004$ & $p=0.026$ \\
& $r=0.43$ & $r=0.01$ \\
Basleine fluctuations & $n=17$ & $n=19$ \\
& $p=0.044$ & $p=0.476$ \\
& $r=0.63$ & $r=0.05$ \\
& $n=17$ & $n=19$ \\
& $p=0.044$ & $p=0.422$ \\
\hline
\end{tabular}

Table 5.8: Pearson correlation coefficients between skin conductance variables and subjective pain ratings for controls and CLBP patients.

\section{DISCUSSION}

This study adressed four questions: (1) are there baseline physiological differences between CLBP patients and controls; (2) do CLBP patients respond differently 
physiologically to pain stimuli; (3) are there differences between CLBP patients and controls in habituation of the physiological responses to pain; and (4) is there a relationship between subjective pain ratings and psychophysiological activity? These questions will be discussed seperately.

\section{Baseline differences between patients and controls}

A sustained increase in muscle tension, hypothesized by the reflex-spasm model, was not found. Although in the past, baseline differences in muscle tension have been reported, reviewing the literature shows that there are quite a large number of studies with negative results (Dolce \& Raczynski, 1985; Flor \& Turk, 1989; Feuerstein et al., 1987; Kravitz et al., 1981). However, as Biedermann has emphasized, surface measurement of EMG is a rather crude measurement, and absolute values do not tell us much (Biedermann, 1984; Biedermann, 1986). Inter-individual comparisons are difficult, and conclusions should be made with caution.

Differences did appear on skin conductance variables. This is in agreement with a previous report of Collins et al. (1982). When skin conductance is taken as an arousal measure, CLBP patients appear to be more aroused. In addition, this is accompanied by a higher levell of subjective anxiety in the CLBP group. The anxiety measures and both baseline SCL and fluctuations were moderately, but significantly, correlated (between 0.27 and 0.36 ). Whether arousal and/or anxiety have an etiological function in CLBP or whether they are a consequence of CLBP cannot be determined.

\section{Differences in psychophysiological responses to pain stimuli}

The observed reaction pattern to the pressure pain stimulus was as follows. The total number of skin conductance fluctuations increased, respiration rate increased and heart rate variability decreased. SCL did not increase, nor did frontal muscle tension. Lumbar muscle tension increased only for back pain patients.

The HR response was not very clear. There was no increase from prestimulus to stimulus period, but there was a decrease from stimulation to recovery. But even this was not very dramatic, only a few beats per minute. The absence of clear accelerative effects might be due to the duration of the stimulus. Compensatory mechanisms become activated, which can decrease the sympathetic response (Sternbach, 1968; Myrtek \& Spital, 1986). In addition, the second by second analysis did not lead to the identification of phasic accelerative responses. With short duration aversive stimuli, these have been shown to occur. The first short latency acceleration is regarded as a reflection of a startle response, while a longer latency accelerative response is regarded as the defensive reaction, which is specific for high intensity stimulation (Turpin, 1986). However, because of the gradual increase in intensity during the pressure pain stimulus, startle probably did not occur. A short phasic defensive response was also absent, and this might be due to the continuous nature of the pain stimulus.

A difference in responding between CLBP patients and controls appeared in the skin conductance variables: patients reacted to stimulation with a greater increase in the 
number of skin conductance fluctuations, which were in addition of greater maximal amplitude. This again might be due to a higher general arousal of CLBP patients. Stronger skin conductance responses to stressful stimulation (cold pressor test and mental arithmetic) in CLBP patients have also been reported by Collins et. al. (Collins et al., 1982). The cardiac response and the repiratory response were not different for the two groups.

The most interesting result was the strong lumbar EMG response which appeared in the patient group. This seem to be in line with the predictions from the diathesis stress model: a specific muscular response occurs, as a result of personally relevant stress (in this case: a pain stimulus). However, there are a few factors which render this interpretation unlikely. Firstly, why were only the muscles on the left side involved in the majority of cases? The pain complaints were not specifically located on the left, but mostly bilaterally or medially. Secondly, the increase in muscle tension occurred as soon as the subject placed his finger in the device, and it disappeared only when the finger was withdrawn. Thus, it did not coincide with the actual pain stimulation. An alternative explanation is that the muscle contractions were caused by the altered position of the subject. It has been suggested that some back pain patients strain their back muscles as some kind of reflexive response when other muscles are used. Kravitz et al. (1981) found that while resting EMG levels did not differ, during the sustained voluntary contraction of various muscle groups (arms, shoulders, legs), back pain patients had higher levels of tension in the lower back than controls. Janda (1978) also reported that back pain patients activated many more muscles than expected in the execution of various movements. In this case the subject had to extend one of his arms (this could also explain the unilaterality of the phenomenon).

An earlier observation about this phenomenon comes from Holmes \& Wolff (1952). They report: "The subject was instructed to elevate the right upper extremity and grasp and squeeze with his right hand for ten seconds the examiner's finger ...... In the execution of such a simple motor function, which might easily have been performed by the skeletal muscles of one extremity and shoulder gindle, this patient called into play muscle groups distant from the right arm which could neither take part in, nor facilitate, the action. The generalized participation of uninvolved muscle groups noted in this experiment was a striking feature in all the backache patients observed."

The biomechanical model of CLBP suggests that patients might be using their muscles differently. The finding of the present study seems most consistent with this model.

\section{Differences in thabituation of psychophysiological responses}

Although differences in habituation between CLBP patients and controls have occurred with subjective pain indicators (notably pain threshold and pain tolerance) we were not able to demontrate this on psychophysiological variables. In this study, habituation of the physiological responses was not present for most variables, neither for patients nor controls. Previous studies have shown that habituation to pain is possible, although it seems to be more difficult than to non-painful stimuli and usually the responses are not abolished completely (Badia, \& Harley, 1970; Condes-Lara et al., 1981; Ernst et al., 
1986; LeBlanc \& Potvin, 1966; Mumford, 1965; Strempel, 1976). In the present study, only the number of skin conductance fuctuations decreased after repeated trials. Other studies have found habituation to pain stimuli for skin conductance variables, EEGvariables, blood pressure and heart rate responses, while the results with EMG variables are mixed (Badia, \&arley, 1970; Condes-Lara et al., 1981; Arntzet al., 1991; Turpin, 1986; LeBlanc \& Potvin, 1966; Strempel, 1976; Turpin \& Siddle, 1978).

There are some marked differences between the habituation task in the present study and those usually employed. First, the stimulus duration was much longer than usual. Also, the interval time between the stimuli was longer. In addition the subject's attention was drawn to the stimulus, he had to indicate when it became painful. Usually subjects are instructed not to pay attention to the habituating stimulus. Lastly, only 6 stimuli were presented, whereas in the past sometimes large numbers of stimuli have been used (Strempel, 1976). The combination of these factors might have prevented the occurrence of more extensive habituation.

To return to the differences between CLBP patients and controls, for the one variable that did habituate, skin conductance fluctuations, no difference was found. It might be that only specific components of the pain reaction (e.g. the behavioral and subjective components) are resistent to habituation, as found in previous studies. Arntz et al. (1991) who used a more common habituation paradigm, also did not find different psychophysiological habituation for CLBP patients. Both groups habituated at the same rate to some of the physiological variables, or they did not habituate at all.

Thus, the slower physiological habituation found by Ellertsen and Hammerborg and Passchier et. al. with migraine patients could not be replicated with CLBP patients. A possible reason might be that the effect is more specific: auditory stimuli can be assumed to be more aversive to headache patients than to headache free controls, and this difference in perceived aversiveness might have caused the slower habituation. The pain stimuli used in this study, and the Antz et. al. study, were not especially burdensome for CLBP patients, at least no more so than for control subjects.

\section{Comparison between subjective and psychophysiological responses}

For control subjects, the perceived painfulness of a stimullus seemed to be related to their overall arousal. Because pain ratings also correlate with baseline arousal, it might be speculated that when subjects are more aroused, they experience more pain, or are more inclined to report pain.

Interestingly, this consistent correlation pattern did not occur with CLBP patients. In a previous article (Peters et al., 1989) we have speculated that the reaction of CLBP patients to acute pain stimuli can be influenced by generalization of their chronic pain behavior. Patients tended to react to every stimulus in the same way, and thus showed no subjective thabituation. Perhaps the absence of a correlation between subjective pain responses and physiological arousal is also a reflection of this: the reponses of CLBP patients to acute pain are more under the control of a stereotyped response manner, than of actual pain intensity. 


\section{Conclusions and suggestions for future research}

The CLBP patients were more aroused and anxious than normal controls.

No evidence was found for the reflex-spasm theory: CLBP patients did not have a sustained increase in back muscle tension. In addition, no evidence was found for the stress reaction or diathesis-stress model of CLBP: back pain patients did not show a greater physiological responsiveness to the pain stimulus. However, some CLBP patients did have an inappropriate activation of their back muscles while changing the position of the arm. This interpretation seems consistent with the biomechanical model of CLBP. Future research should try to further establish the relationship between alteration of position and back muscle reaction, without the interference of a stress task.

No evidence of a deviant physiological habituation pattern for CLBP patients was found. However, the paradigm used does not allow the drawing of firm conclusions, because even in the control group no strong habituation effects were induced. But in the light of a previous experiment there is, at the moment, no cause to assume that there is a difference in the physiological habituation to a pain stimulus. Future research can perhaps elucidate which reactions show deviant habituation and under what conditions.

The pain ratings of CLBP patients were less related to arousal than the ratings of controls. They might be more under the control of response tendencies. This can be verified further by introducing real variation in stimulus intensity, and seeing whether the pain ratings of controls follow the actual intensity more than the ratings of CLBP patients do. 


\subsection{DIFEERENCES BETWEEN CLBP PATTENTS AND CONTROLS IN THE PERCEPTION OF EXPERIMENTAL PAIN STIMULI OF VARIOUS INTENSITIES}

\section{INTRODUCTION}

It has frequently been suggested that patients who experience continuous pain have an altered perception of experimental pain stimuli (Naliboff \& Cohen, 1989). The nature of this difference, either increased or decreased sensitivity, seems to be dependent on the particular variable which is assessed. Pain threshold was often found to be higher for chronic pain patients (Wolskee \& Gracely, 1980; Naliboff et al., 1981; Cohen et al., 1983; Yang et al., 1985; Merskey \& Evans, 1975; Hazouri \& Mueller, 1950), whereas pain tolerance was often lower (Schmidt \& Brands, 1986; Brands \& Schmidt, 1987; Peters et al., 1989; Molin et al., 1973). The intensity of the experimental pain stimulus might be the crucial factor in determining whether a decreased or increased sensitivity is found.

We have previously speculated that two separate processes could be responsible for altered pain responsiveness in chronic pain patients, working in opposite directions. The first process is one which increases the sensitivity for acute pain stimuli. It is assumed to be based on the functioning of a psychological mechanism which we have called "response tendencies". Response tendencies are assumed to affect pain sensitivity at all levels of stimulus intensity. However, at low intensity pain levels a second process which decreases pain sensitivity, is proposed to operate simultaneously, and this would antagonize the effect of response tendencies. This pain decreasing process is possibly based on a perceptual/psychological mechanism (described in terms of the adaptation level theory; Rollman, 1983; Gracely, 1984) or on a physiological mechanism (DNIC; LeBars \& Willer, 1988). We have also speculated that with weak to moderate pain stimuli the effect of the pain decreasing process will predominate, but that at a higher pain level this process will loose it efficacy, and response tendencies become more important.

Two different psychological mechanisms can be speculated to form the basis of response tendencies. Firstly, it could be based on operant learning processes. Complaining about pain is an aspect of pain behaviour presumably under operant control: it can elicit positive remforcement from the environment (Fordyce, 1979). Once established, this behaviour can generalized from the clinical situation to the experimental pain situation (Schmidt \& Brands, 1986), leading to an inclination towards reporting pain sooner than other subjects would. An example of stch generalized pain complaining behaviour in chronic pain patients was given by Cleeland (1989). He demonstrated that when patients had to rate situations of various degrees of potential painfulness on a visual analog scalle, their responses showed little variation. They consistently scored each situation as "highly painful". 
Secondly, response tendencies could also be based on hypervigilance, which is characterized by a greater awareness of proprioceptive signals, in conbination with a more threatening cognitive interpretation of these signals. Hypervigilance increases the saliency of normal bodily sensations, decreasing the threshold for a sensation to come into awareness, and leading to the perception of a sensation as more intense. It has been proposed that chronic pain patients are prone to displaying bypervigilant behaviour (Feuerstein et al, 1987). This would imply that chronic pain patients will not only report more pain, but also a greater amount of non-specific bodily sensation. In fact, this is what has been found in chronic low back pain patients (Schmidt et al., 1989).

The principal aim of this study was to test the hypothesis that the nature of the difference in pain responsiveness between chronic pain patients and controls depends on the intensity of the experimental pain stimulus. It was tested whether chronic pain patients perceive low intensity pain stimuli as less painful and high intensity pain stimuli as more painful than healthy controls. The responses to electrical stimuli of various intensities were measured. Detection threshold (DT), pain perception threshold (PPT) and maxinal pain tolerance (MPT) were determined and subsequently, subjects rated the painfulness of stimuli of intermediate intensities.

A second aim was to start with elucidating the proposed mechanisms responsible for the differences in pain sensitivity between chronic pain patients and controls. The first aspect considered was whether response tendencies could be found in chronic pain patients, and whether these were due to increased complaining as a result of generalization of operant learning processes. To test whether chronic pain patients do in fact show a higher tendency to report sensations in general, the response to fake stimulation was measured. This operationalization was chosen because it prevented the confounding of genuine augmented perception of sensations (resulting from hypervigilance) with increased reporting of sensations.

Finally, a questionnaire measuring the presence of all kinds of bodily sensations was administered (somatization scale).

In short, the following hypothesis are tested:

1. CLBP patients have a higher PPT than normal controls

2. CLBP patients have a lower MPT than normal controls

3. The pain intensity ratings of CLBP patients are lower than those of controls at low stimulus intensities, but higher at high stimulus intensities.

4. CLBP patients report more often, or sooner, a sensation after fake stimulation

5. CLBP patients score higher on a questionnaire measuring somatization.

DT was included as an exploratory variable, no specific predictions were made. Both increased and decreased DT can be hypothesized on theoretical grounds, determined either by the operation of response tendencies below a painful level, or by decreased physiological sensitivity because of DNIC. 


\section{METHOD}

\section{Subjects}

Forty subjects participated in the study, 20 CLBP patients and 20 controls, matched for age and sex (see table 5.9). CLBP patients were recruited by means of an advertisement in a local newspaper. The selection criteria for CLBP patients were: (1) a history of at least one year of low back pain, (2) no known chronic somatic illnesses, related to back pain or otherwise, and (3) continuous pain.

Partiners of the patients, their relatives and acquaintances served as control subjects. Controls were screened for the absence of chronic or acute pain. All subjects completed a questionnaire on prior illnesses, daily activities, present health and use of medication, alcohol and cigarettes. CLBP patients, in addition, complleted a back pain questionnaire. Both questionnaires were screened by a General Practitioner before subjects were admitted to the study. The use of analgesics within 24 hours before the study was not permitted.

\begin{tabular}{lccc}
\hline & $n$ & mean age & range \\
\hline $\begin{array}{l}\text { CLBP patients } \\
\text { male } \\
\text { female }\end{array}$ & 10 & 46.2 & $\begin{array}{l}(30-55) \\
(21-51)\end{array}$ \\
$\begin{array}{l}\text { Controls } \\
\text { male } \\
\text { fernale }\end{array}$ & 10 & 41.0 & \\
\hline
\end{tabular}

Table 5.9: Age and sex distribution of the subjects.

\section{Appararus}

Elecirical stimuli were delivered by a Siemens current generator (type neuroton 627), approved for application with human subjects. Output intensity could range from 0 to 12 $\mathrm{mA}$, in steps of $0.1 \mathrm{~mA}$. Each stimulus had at duration of 4 seconds and consisted of 20 msec square puises, with an internal frequency of $20 \mathrm{~Hz}$. Two kinds of electrodes were used to deliver the stimuli. The first was a concentric shock electrode made of stainless steel. Contact with the skin was made through pads of cellulose, soaked in an isotonic saline solution (Tursky, 1973). Secondly, a pair of Beckmann $8 \mathrm{~mm}$ Ag/AgCl electrodes filled win Hewlet Packard Redux creme was used. This electrode pair produced more disconfort at the same current intensity than the concentric electrode, and it was used for measuring PPT and MPT. To measure DT, the concentric electrode was used, because it allowed for better discrimination at low stimulus intensities. The concentric shock electrode was attached to the degreased skin at the lateral side of the ankle. The 
pair of Beckmann electrodes was attached to the same location on the other leg, with the anode and cathode always in the same relative position.

\section{Procedure}

Before the start of the experiment the subjects completed the somatization subscale of the NVM, a dutch shortened version of the MMPI. The somatization scale correlates 0.91 with the originall MMPI subscale hypochondriasis, and consists of 20 items concerning non-specific bodily sensations and complaints (e.g. dizziness, sweating, disturbed sleep). The experiment itself took place in a soundproof and temperature controlled laboratory room. Subjects were seated at a table in a corner of the room. The session consisted of the following sequence of tests:

1. response tendencies

2. Detection threshold for the electrical stimulus

3. PPT and MPT measured in an ascending series of shocks

4. VAS ratings of electrical shocks of various intensities presented in random order

5. PPT measured in a descending series of shocks

6. response tendencies

Response tendencies were operationalized in the following way. Subjects were told that they would receive 5 trials during which very weak electrical stimuli would be presented and that their ability to detect such faint signals would be tested. They were told they would probably feel nothing at first, but that the intensity would gradually increase. As soon as they became aware of any sensation at all, they had to say so, and stimulation would be stopped. It was stressed that it concerned very faint stimuli. In reality, no stimulation was actually given. During the test, subjects were blind-folded. The experimenter stayed in the room with the subject, warned him/her that stimulation would begin, and started the clock. When the subject reported to have felt a sensation, the clock was stopped, and the time was recorded. The maximum duration per trial was 60 seconds. When no sensation was reported the subject was given the maximum score of 60 . The subject was instructed that a new trial would begin and the procedure was repeated. A total of five 1 -minute trials was given.

During the next test (DT), subjects remained blind-folded. They were told that again very weak electrical shocks would be presented. During this test, stimulation was actually given. Shocks started at $0.0 \mathrm{~mA}$ (no shock) and increased in steps of $0.1 \mathrm{~mA}$. The intensity at which a stimulus was perceived for the first time was recorded. To measure PPT and MPT, the subject had to concentrate on the other (right) leg. Stimulation again started at $0.0 \mathrm{~mA}$ and increased in steps of $0.1 \mathrm{~mA}$. Subjects had to indicate when they first experienced the shock as painful (PPT) and when they wanted to stop the series (MPT).

During test 4 , all stimulus intensities below MPT for a given subject were presented in random order. Thus, the number of stimuli a subject received varied, depending on his/her MPT (average number: 20 , range: $8-43$ ). Subjects had a booklet in front of them, in which they scored every shock for its perceived painfulness. There were three scoring 
caltegories. The first was: "no shock felt", the second: "shock felt, but not painful" and the third: "painful shock". When a stimulus was scored as painful, the intensity of the perceived pain had to be rated on a $10 \mathrm{~cm}$ VAS scale (with the extremes: minimal pain maximal pain).

Next, a descending series of shocks was presented. The series started at an intensity well above the previously established PPT, but below MPT. Stimulus intensity was decreased in steps of $0.1 \mathrm{~mA}$, and the subject had to indicate when the shock was no longer perceived as painful. Finally, another response tendency test took place. The procedure was the same as before, except that this time it was suggested that the shock would be given through electrodes on the finger. Again, no current was actually delivered. After the completion of all tests, the electrodes were removed and the subjects were paid for participation. The entire procedure took about 1.5 to 2 hours to complete.

\section{Data reduction and analysis}

A total of 10 scores of response tendencies was obtained, 5 times during test 1 , and 5 times during test 6 . Every score could range between 0 and $60 \mathrm{sec}$. The results of the two tests were analyzed together. Two summary scores were calculated: (1) a count of the total number of occasions on which a sensation was reported by an individual (0-10), and (2) a summation of all 10 durations (in seconds) before a sensation was reported ( 0 600).

DT, PPT and MPT were expressed in terms of the current intensity at which they occurred. A mean PPT was calculated on the basis of the two separate (ascending and descending) assessments. The VAS scores of the randomly delivered pain stimuli of each subject were used to construct a pain perception curve for each subject. This curve plotted the VAS score as a function of stimulus intensity. The linear component of a curve can be approximated by means of a linear regression analysis. The alpha coefficient in the regression equation indicates the slope of the regression line, and thus the rate which pain report increases as a function of stimulus intensity. Because it is hypothesized that CLBP patients become aware of pain later, but report intolerable pain sooner, the slope of their curve is expected to be steeper.

Most comparisons between the two groups were made by means of t-tests. However, when the distribution of a variable deviated significantly from normallity, a nonparametric test was employed (Mann-Whitney test).

\section{RESULTS}

\section{Pain sensitivity}

DT, PPT (separately for the ascending and descending series, and mean) and MPT for CLBP patients and controls are shown in table 5.10. Differences were tested by 
means of t-tests for independent groups. Hypotheses 1 and 2 could not be confirmed. CLBP patients did not have a higher PPT (ascending, descending, or mean) or a lower MPT. Both CLBP patients and controls had a lower PPT in the first ascending series than in the second descending series. (mean: $1.61 \mathrm{~mA}$ vs. $2.53 \mathrm{~mA}, \mathrm{t}=-3.61$, $\mathrm{df}=39$, $p=0.001$ ). This could be either the result of the different procedure, or more likely a repetition effect.

Analysis of variance showed that there was also a significant sex effect on the first (ascending) PPT, mean PPT and MPT. Male subjects scored higher on these variables. There was no group by sex interaction.

DT is also shown in table 5.10. The difference between the groups was tested nonparametrically (Mann-Whitney test). DT did not differ significantly between the groups, although there was a trend towards a higher DT for CLBP patients $(\mathrm{z}=-1.81, \mathrm{p}=0.072$, two-tailed).

Hypothesis 3 predicts a steeper slope of the pain perception curve for CLBP patients. In order to construct a curve for an individual, at least three data points had to be available, meaning that at least three stimull during the random series had to be scored as painful. However, often the VAS scores did not exceed a very faintly painful level, and a curve covering the whole range of pain perception could not be constructed. Some subjects even rated none of the stimuli of the random series as painful, while others rated only one or two to be painful. Therefore, the intended construction of pain perception curves was not possible and hypothesis 3 could not be tested.

All in all, we could not confirm the predicted differences in pain sensitivity between CLBP patients and controls.

\begin{tabular}{|c|c|c|c|c|c|}
\hline & \multicolumn{2}{|c|}{ controls } & \multicolumn{2}{|c|}{ CLBP patients } & \multirow[b]{2}{*}{ signif. } \\
\hline & $\begin{array}{l}\text { mean } \\
\text { (sd) }\end{array}$ & $n$ & $\begin{array}{l}\text { mean } \\
\text { (sd) }\end{array}$ & n & \\
\hline DT & $\begin{array}{l}0.60 \\
10.641\end{array}$ & 20 & $\begin{array}{l}1.18 \\
(1.64)\end{array}$ & 20 & $p=0.072$ \\
\hline ascending PPT & $\begin{array}{l}1.72 \\
10.991\end{array}$ & 20 & $\begin{array}{l}1.50 \\
(0.82)\end{array}$ & 20 & $p=0.45$ \\
\hline descending PPT & $\begin{array}{l}2.85 \\
(2.63)\end{array}$ & 19 & $\begin{array}{l}2.24 \\
(1.08)\end{array}$ & 20 & $p=0.36$ \\
\hline mean PPT & $\begin{array}{l}2.25 \\
(1.63)\end{array}$ & 19 & $\begin{array}{l}1.87 \\
(0.93)\end{array}$ & 20 & $p=0.32$ \\
\hline MPT & $\begin{array}{l}4.22 \\
(3.04)\end{array}$ & 20 & $\begin{array}{l}3.26 \\
12.18 \mid\end{array}$ & 20 & $p=0.13$ \\
\hline
\end{tabular}

Table 5.10: Mean values and standard deviations of DT, PPT and MPT for CLBP patients and controls. Only MPT was tested with a one-tailed significance level, because the result appeared to be in line with the prediction. All other variables were tested two-tailed. 


\section{Response tendencies and somatixation}

In both groups, eight subjects reported to have felt something on one or more occasions. The eight patients reported a mean number of $2.8(\mathrm{sd}=1.8)$ sensations, and the eight controls a mean number of $4.1(\mathrm{sd}=3.0)$, out of the possible maximum of 10 sensations. This did not differ significantly $(t=-1.11, \mathrm{df}=14, \mathrm{p}=0.29)$. The groups also did not differ on the total duration before a report of sensation was made. Summation of the duration of all measurement periods, yielded a mean score of $547.4 \mathrm{sec}$ $(\mathrm{sd}=101.2)$ for the CIBP patient group, and $570.4 \mathrm{sec}(\mathrm{sd}=53.4)$ for the control group. Again, the difference was not statistically significant (tested non-parametrically, because the distribution was markedly skewed, most subjects scored $600 . \mathbb{Z}=-0.26, p=0.80$ ).

On the somatization subscale a large difference between the two groups was found (mean 3.6 $(\mathrm{sd}=2.9)$ for controls, mean 12.1 $(\mathrm{sd}=6.1)$ for CLBP patients). This difference was statistically significant $(t=-5.63, \mathrm{df}=39, \mathrm{p}<0.01)$. When comparing the scores of the two groups with the norm tables, controls score in the category "average" when comparing them to a general population, while CLBP patients score in the category "high" ( 7 categories are availlable: very low, low, below average, average, above average, high and very high). Compared to the nom tables of (psycho) somatic patients, CLBP patients score in the category "average".

Subjects who showed a response tendency on one or more occasions did not score higher on the somatization subscale compared to subjects without response tendencies (mean: 8.56 for the response tendency group, and 7.69 for the no response tendency group, $t=-0.36, p=0.72$ ).

\section{DISCUSSION}

The principal aim of this study was to test the hypothesis that in chronic pain patients decreased sensitivity is found for low intensity experimental pain stimuli, and increased sensitivity for high intensity pain stimuli. We were not able to confirm this hypothesis. No differences in PPT or MPT between CLBP patients and controls were found. The hypothesis conceming the pain perception curves of CLBP patients and controls could not be tested. However, in view of the absence of a difference on PPT and MPT, differences in the slope of this curve seem unlikely.

Thus, our patient group did not show a different responsiveness to an electrical pain stimulus (although there seems to have been a trend towards higher DT's in CLBP patients).

As outlined in the introduction, previous research has repeatedly shown a higher PPT and a lower MPT for chronic pain patients, and also specifically for CLBP patients. Why did we not find this in the present study? A plausible explanation may be that this was due to the pain stimulus used. In previous studies, either thermal (Hazouri \& Mueller, 1950; Wolskee \& Gracely, 1980; Naliboff et al, 1981; Cohen et al., 1983; Yang et al., 1985; Lipman et al., 1987), cold (Schmidt \& Brands, 1986; Brands \& Schmidt, 1987) 
or mechanical (Merskey \& Evans, 1975; Malow et al, 1980; Peters et al, 1989) stimuli have been employed, whereas this study used electrical stimuli. Electrical stimuli are easy to apply, and reliably quantified in terms of current intensity. However, there are some disadvantages to using electrical shocks. Shocks are often fear-inducing and frequently experienced as "aversive" rather than "painful". Many of our subjects expressed difficulty in calling the sensation they felt "pain". When subjects become used to the sensation, and probably the initial fear wears of, PPT and MPT can increase considerably ${ }^{1}$. Most subjects are probably more familiar with the sensations of mechanical and thermal pain, hence, these stimuli will induce less initial fear and the threshold increase over trials will probably be less.

One previous study has used electrical pain stimulli to assess pain responsiveness of chronic pain patients (Molin et al., 1973). In fact, this was one of the few studies which found a contradictory result: a lower PPT in the patient group (MPT was not significantly different). Perhaps sensitivity for electrical stimuli is not a good indicator of pain sensitivity.

The secondary aim of the study was to test for the presence of increased response tendencies in CLBP patients, as a result of generalized complaining behaviour. We were unable to show that CLBP patients displayed these kinds of response tendencies to a greater extent than controls. On the other hand, CLBP patients did complain more about non-specific bodily sensations, as reflected on the somatization subscale. The conclusion of this might be that response tendencies as a result of increased complaining behaviour established by operant conditioning do not play a prominent role in CLBP patients. The alternative mechanism, response tendencies resulting from hypervigilance, is perhaps more likely. In both groups some subjects displayed response tendencies. Post hoc, it might be argued that during the response tendency test, a kind of hypervigilance was experimentally induced in these subjects. Oosterlaan \& Schmidt (unpublished data) have demonstrated that the combination of attention and suggestion can lead to the report of a substantial number of non-specific bodily sensations in healthy students. In the present experiment, subjects were instructed to attend closely to the foot, and the occurrence of sensations was suggested. Without specific instructions, it might well be that only CLBP patients are hyper-attentive to bodily symptoms.

Thus, although we have not directly demonstrated it in this experiment, hypervigilance seems more likely to cause response tendencies than the generalization of complaining behaviour, at least with low intensity sensations. However, increased complaining could be important in determining responsiveness in chronic pain patients when high intensity (painful) sensations are assessed. Based on the stimulus generalization hypothesis, it can be argued that increased complaining specifically occurs with painful stimuli. Thus, our inability to find a response tendency could also be the result of the way in which this variable was operationalized.

\footnotetext{
'After this study was carried out, a report of Watkins 11989$)$ appeared, in which it was observed that as subjects undergo training with an electrical pain stimulus, the PPT can increase more than $300 \%$ and the MPT more then $350 \%$.
} 
In conclusion, although this sudy did not confirm our predictions, more research is needed. Because response tendencies are difficult to operationalize, and hypervigilance and generalized complaining behaviour are not easily separated experimentally, our future studies will first concentrate on the other aspect of the model, the process which decreases pain responsiveness in chronic pain patients.

It is important to improve upon the reliability of the pain sensitivity assessment procedure. Other pain stimuli besides electrical ones should be used, and when using an electrical stimulus, it should preferentially be preceded by a training period, to rule out inflation of pain scores due to liear evoked by non-familiarity. Also, a pain discrimination task (which specifically measures the sensory component of pain perception) can be applied. 


\subsection{IS THERE EVIDENCE FOR COGNITIVE- PHYSIOLOGICAL DISCORDANCE AND DESYNCHRONY IN CLBP PATIENTS?}

\section{INTRODUCTION}

Lang (1971) has proposed that "emotions" are comprised of multiple response systems, namely a verbal-cognitive system, a motoric-behavioural system and a physiological system. These response systems are partly independent, in some circumstances all three systems will be activated alike, whereas in other circumstances they function at diverging levels of activity. The degree of agreement between the response systems possibly varies between individuals. The concepts of concordance and discordance have been used to denote whether variables assumed to measure the same process, but belonging to different response systems, agree or disagree. Concordance means that there is agreement between these variables, whereas discordance indicates disagreement between these variables. Two related terms have also been used, namely synchrony and desynchrony. These terms indicate whether variables from different response systems change in a parallel way. For instance, when after repetitive presentation of an emotional stimulus the physiological correlates of the emotion die down, but the subjective experience of the emotion persist, the physiological and cognitive response systems are said to change desynchronously.

Concordance/discordance and synchrony/desynchrony have mainly been applied in the field of anxiety (Hodgson \& Rachman, 1974). Several factors have been identified which can cause response systems to react in an increased vs. decreased concordant and synchronous way. These are for instance the intensity of an emotion and the level of demand on a subject in a particular situation.

Gannon \& Haynes (1986) have hypothesized that discordance between the physiological and the cognitive response system can be clinically significant in that it may contribute to the development and maintenance of psychosomatic complaints. Lack of awareness of the physiologicall processes which take place within the body or of the causal relationship which lead to these processes, can prevent the initiation of appropriate coping behaviours. Sexual disorders, insomnia and chronic recurrent headache are mentioned as possible consequences of this cognitive-physiological discordance. Discordance and desynchrony between the cognitive and physiological response system could also apply to the situation in which an individual reports all kinds of bodily symptoms, without the presence of sufficient physiological activity to explain these symptoms. Such a situation of apparent over-reporting of symptoms can arise as a result of learning processes. The physiological and the cognitive response system will be subjected to disparate learning processes and social contingencies. For instance, physiological reactions may habituate after repeated stimulation, whereas 
subjective symptom reporting increases at the same time because of positive reinforcement.

Cognitive physiological discordance can therefore denote the predominance of subjective symptom awareness and reporting over physiological responsivity, or alternatively, a situation of predominance of the physiological response system over the cognitive response system. Both types of cognitive-physiologicall discordance could be associated with chronic pain syndromes. Gannon and Haynes assume that their version of the cognitive physiological discordance concept is related for instance to chronic recurrent headaches. In section 2.6 it was speculated that the mechanism envisaged by these authors could also play a role in other chronic pain syndromes, for instance chronic low back pain (CLBP). The alternative form of cognitivephysiological discordance, with the predominance of cognitive and behavioural factors over nociceptive physiological activity, has formed the basis of the "fear-avoidance model of exaggerated pain perception" (Lethem et al., 1983; Slade et al., 1983). This model argues that when individuals avoid rather than confront pain, out of fear of pain, the affective-motivational component of pain and consequently pain behaviour can become out of synchrony with the physiological basis of pain. This can cause pain to persist after the healing of the original injury.

In section 2.6 it was described what difficulties may arise when the discordance hypothesis is tested in a clinical situation. A plea was made for laboratory evaluation. This can be done by measuring the association of physiological and cognitive responses to experimental pain stimuli. Such a procedure has the advantage that the physiological reactions to an experimental pain stimulus can easily be identified and measured. The physiological response to experimental pain stimuli generally consists of cf. increased skin conductance, heart rate acceleration (or a bi-phasic response with an accelerative and a decelerative component), peripheral vasoconstriction, increased respiration rate and increased muscle tonus. The magnitude of the subjective experience of pain can be compared with the magnitude of the physiological responses to the stimulus. Rabavilas et al. (1982) have conducted a similar laboratory experiment with patients with involutional depression. It was demonstrated that these patients showed significantly less synchronous changes between the autonomic (SCR, heart rate and finger plethysmography) and subjective response system when stressful stimuli were presented.

Data collection in two previous experiments (Peters et al., 1989; Arntz et al., 1991) allowed us to investigate the discordance/concordance and the (de)synchrony of pain responses in CLIBP patients in more detail. In the first part of this paper, concordance between skin conductance responses and subjective pain scores after electrical stimuli of different intensity will be determined for CLBP patients and painfree controls. In addition, the agreement between objective stimulus intensity and perceived pain intensity will be assessed, in order to test whether there is any indication that CLBP patients perceive pain stimuli less accurate, and cannot differentiate between the various intensity levels as good as pain-free subjects. In section 5.2 it was argued that the (experimental) pain responses of CLBP patients are possibly more determined by stable response tendencies than by actual stimulus intensity. If this is in fact so, the correlation between VAS intensity scores and 
objective stimulus intensity would be low. In the second part of this paper, the synchrony of changes in the physiological and the cognitiwe response systems after stimulus repetition is assessed for CLBP patients and controls. The change in perceived pain intensity after 20 repetitions of a constant intensity elactrical shock is compared with the changes of several physiological variables (SCR, heart rate, vasoconstriction, frontalis and paraspinal muscle tension). The agreement of the changes between the various variables within the physiological system is also assessed.

\section{Experiment 1: concordance/discordance of subjective pain ratings and skin conductance responses}

\section{METHOD}

Subject were 20 male CLBP patients and 20 male pain-free healthy controls. Details of subject characteristics are given in section 5.3. All subjects participated in several tests, one of which was to score the painfulness of a series of electrical stimuli of varying intensity. Stimuli were delivered by means of a pair of Beckman. $\mathrm{Ag} / \mathrm{AgCl}$ electrodes, taped to the right ankle. The duration of each shock was 4 seconds. The number of shocks which was delivered varied between subjects, and depended upon an individual's pain tollerance. All intensities between $0.1 \mathrm{~mA}$ and the previously established pain tolerance were presented in random order, in steps of 0.2 $\mathrm{mA}$. The mean number of stimuli received by a subject was 29 (range 8-41). After each stimulus, the perceived painfulness was scored in a booklet. Three categories were available: the first category was marked "stimulus not perceived at all", the second category was marked "stimulus perceived, but not experienced as painful", and the third category was marked "painful stimulus". When a stimulus was scored in the last category (painful stimulus), the subject in addition had to rate the pain intensity on a $10 \mathrm{~cm}$ VAS, marked "no pain" and "unbearable pain" at the extremes. Afterwards, all scores were rank-ordered, with the first category receiving the lowest rank, the second category the next lowest rank, and scores in the last category a rank according to their rating on the VAS-scale. Ties were ranked in the usual manner, by assigning them the average rank score of all observations within the tie (Siegel, 1956).

Skin conductance recordings were made from the medial phalanx of the second and third finger by means of $\mathrm{Ag} / \mathrm{AgCl}$ Beckman electrodes filled with isotonic conductance cream. Recording took place beginning 4 seconds before each shock, until 6 seconds after the shock. Scoring was done manually, by measuring the deflection on the write-out in millimeters. These scores were also rank-ordered, the smallest deflection receiving the lowest rank.

Agreement between the orderings of the subjective pain ratings and of the SCRs 
was assessed by means of Spearman rank correlation coefficients. The formula to correct for a large number of wed observations given by Siegel (1956, pag. 207) was applied, because especially the subjective pain scores were frequently tied. The two lower categories indicating that the stimulus was not perceived at all, or not perceived as painful, were almost always used more than once by a subject. There was an average of 6 tied observations in these categories. Stimuli which were scored as painful and therefore rated on the VAS, were never tied. The correlations between the subjective pain score and stimulus intensity and between SCR and stimulus intensity were also calculated.

\section{RESULTS}

Due to failure of the recording equipment, the data of three subjects were lost. An additional seven subjects were left out of the analysis because they had only scored two (or less) stimuli as painful. In these instances all, or nearly all, scores were tied into the two lower categories and the correlation coefficient could not be calculated reliably. As a result, correlation coefficients could be determined for 13 subjects in the patient group, and 17 subjects in the control group. The three sets of correlations and the number of observations on which they are based, are shown in table 5.11.

Cognitive-physiological discordance is indicated by low correlation coefficients between the pain ratings and skin conductance responses (first column). Below each set of correlations the mean of these correlations is displayed. A $\mathrm{z}$-transformation could not be applied, as Spearman correlations were used. Therefore the mean for untransformed scores was computed.'

Table 5.11 shows that there are no differences between the groups on any of the associations. Discordance between the perceived painfulness of a stimulus and the skin conductance response to this stimulus, indicated by a non-significant or low correlation between these variables, is seen in several subjects in both groups. Subjects who show a high to moderate association between SCRs and pain ratings are also equally distributed over the two groups.

Table 5.11 also shows that the strongest association is between objective stimulus intensity and perceived painfulness of a stimulus. Thus "it appears that subjects reliably perceive the intensity of electrical stimuli of different magnitude. CLBP patients are equally competent in this respect. There is no indication that their subjective response is less related to differences in stimulus intensity because of stable response tendencies.

'Computation of the mean of untransformed correlations, based on an unequall number of data points, may be unacceptable for the professional statistician. However, they have been displayed for illustrative purposes. No further statistical analysis on these means was performed. 
patients:

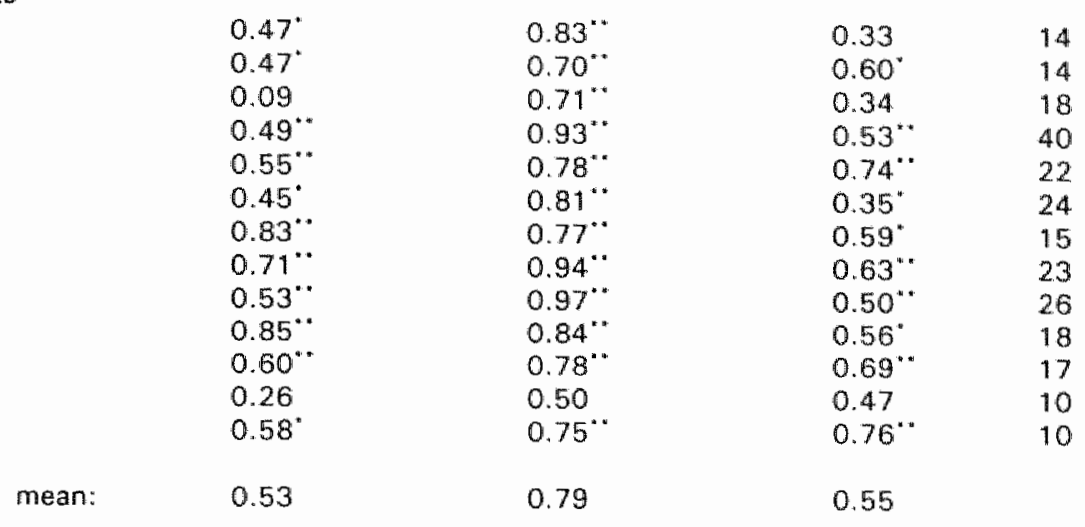

controls

\begin{tabular}{|c|c|c|c|}
\hline $0.56^{*}$ & $0.80^{\circ "}$ & $0.79^{\prime \prime \prime}$ & 12 \\
\hline $0.60^{\circ 4}$ & $0.86^{* \prime}$ & $0.67^{\circ "}$ & 20 \\
\hline $0.74^{+\infty}$ & $0.81^{\circ}$ & $0.73^{\circ "}$ & 12 \\
\hline 0.18 & $0.73^{* *}$ & $0.37^{\circ}$ & 21 \\
\hline-0.02 & $0.68^{\circ *}$ & 0.00 & 25 \\
\hline $0.73^{*}$ & $0.88^{\circ *}$ & $0.78^{\circ "}$ & 10 \\
\hline $0.62^{\circ}$ & $0.91^{\prime \prime}$ & $0.65^{* 0}$ & 41 \\
\hline 0.14 & $0.69^{\circ "}$ & $0.36^{*}$ & 22 \\
\hline $0.80^{\circ}$ & $0.88^{\circ}$ & $0.79^{\prime \prime \prime}$ & 17 \\
\hline 0.26 & $0.82^{\circ "}$ & 0.110 & 17 \\
\hline $0.38^{*}$ & $0.60^{\prime \prime \prime}$ & $0.56^{\circ}$ & 22 \\
\hline $0.86^{\circ *}$ & $0.84^{* \prime \prime}$ & $0.86^{*}$ & 15 \\
\hline 0.24 & $0.80^{\circ \prime \prime}$ & 0.27 & 27 \\
\hline 0.46 & $0.82^{\circ}$ & $0.75^{\circ}$ & 8 \\
\hline 0.13 & $0.90^{\circ}$ & 0.17 & 15 \\
\hline $0.57^{\circ}$ & $0.94^{\circ "}$ & $0.57^{\circ *}$ & 24 \\
\hline-0.20 & $0.83^{\circ}$ & 0.16 & 16 \\
\hline 0.41 & 0.81 & 0.50 & \\
\hline
\end{tabular}

Table 5.1\%: Spearman correlation coefficients between subjective pain score (PAIN), skin conductance responses (SCR) and stimulus intensity $\| \mathrm{NT}$ for every subject. * indicates that the correlation coefficient is significant at $p=0.05$, and " indicates a significance $\|$ vel of $p=0.01$ 
Experiment 2: synchrony/desynchrony between changes in subjective pain score and physiological responses

\section{METHOD}

Subjects were 22 chronic low back pain patients ( 12 male, 10 fermale) and 21 healthy controls ( $11 \mathrm{male}, 10$ female). The mean age was 42.7 years in the patient group and 43.9 years in the control group. For more details on subjects see Amtz et al. (1991).

Electric shocks of 1-sec duration were given to the index finger of the left hand. For each subject, a shock level was established by increasing the amperage from zero up in steps of $0.5 \mathrm{~mA}$, until the subject indicated that the shock was painful. Having reached the pain threshold level, subjects rated each stimulus on a $0-100 \mathrm{~mm}$ VAS. The intensity at which a stimulus was rated as more than 50 on the VAS was used on all subsequent trials. Subjects were told that they would receive an unspecified number of shocks at the level just established. Two series each consisting of 10 stimuli at the pre-established level were given. The mean inter-stimulus interval between the 10 stimuli in each series was $30 \%$ seconds. Between the two series an interval of several minutes took place. After each shock, physiological measures (skin conductance, heart rate, finger pulse volume, frontalis EMG and paraspinal EMG) were obtained. Details on apparatus are reported in the paper by Arntz et al. For each measure, the phasic response immediately following the shock was determined. SCR was defined as the largest deflection superimposed upon skin conductance level occurring 1-4 sec after stimulus onset. HR response was defined as the difference in inter-beat interval between the mean of ten beats pre-shock and the mean of six beats post-shock. Previous analysis of the HR data had shown that the predominant response was a HR acceleration (see Arntz et al., 1991), and only these accelerative responses were included in the analysis. When there was a HR deceleration, this was scored as no response. The finger pulse volume (FPV) response, which measures vasoconstriction, was defined as the percentage of the three smallest deflections in a $15 \mathrm{sec}$ post-shock period compared to the smallest deflection in the $10 \mathrm{sec}$ pre-shock period (Siddle \& Heron, 1975). EMG data were first subjected to a logarithmic transformation, because of a positive skewness of the distribution. Both frontalis and paraspinal EMG responses were defined as the mean $\mathbb{E M G}$ level for 7 seconds after the shock minus the tonic EMG level before shock.

VAS ratings of perceived pain intensity were made after the 10th and the 20st shock. Thus, with the inclusion of the initial VAS rating of the shock level assessment procedure, three successive pain rating were available. The VAS rating of the 10th stimulus (VAS-mid) and of the 20th stimulus (VAS-end) were expressed as percentages of the VAS rating at shock assessment (VAS-begin), creating two new variables: $\triangle V A S 1$ and $\triangle V A S 2$ (resp. the change from begin to half-way the session and from begin to the end of the session). In this way, the change of perceived pain intensity within time was established. Nearly all subjects showed habituation of VAS 
responses. None of the subjects showed sensitization.

In order to assess the change of the physiological responses after stimulus repetition, responsiveness at the beginning of the first series, at the end of the first series, and at the end the second series was assessed for all variables. The average of the responses to the first and second stimulus was used as the indicator of the initial responsiveness, the average of the responses to stimulus 9 and 10 as the responsiveness half-way, and the average of the responses to stimulus 19 and 20 as the final responsiveness. This yielded three scores for every variable. The response half-way and the final responses were expressed as a percentage of the initial response, yielding two new variables for every physiological measure ( $\triangle S C R$ I, $\triangle S C R 2, \Delta H R 1, \triangle H R 2$, etc.). Synchrony between the various variables was established in different ways. First, correlations between $\triangle$ VASl, $\triangle$ SCR1, $\triangle H R 1$, $\triangle F P V 1, \triangle E M G$ frontalis1 and $\triangle E M G p a r a s p i n a l 1$ and between $\triangle V A S 2, \triangle S C R 2$, $\triangle \mathrm{HR} 2, \triangle \mathrm{FPV} 2, \triangle \mathrm{EMGfrontalis2}$ and $\triangle \mathrm{EMGparaspinal2}$ were calculated, for patients and controls separately. These correlations indicate whether the subject showing the strongest habituation on one variable also showed the strongest habituation on a second variable in comparison to other subjects. If CLBP patients have a more desynchronous pattern of change for the various responses, lower correlations between the variables are expected. Most variables did not have a normal distribution, and therefore Spearman rank correlations were computed.

A second method to asses synchrony/desynchrony was to calculate the difference in percentage change for each pair of variables. For instance, whether VAS ratings and SCRs changed in a parallel fashion from the first to the second assessment period can be estimated by the formula: $d=a b s(\triangle V A S 1-\triangle S C R 1)$. Small values of $d$ indicate a change in the same direction, and of comparable magnitude. For all pairs of variables the d's of CLBP patients are tested against the d's of controls. If CLBP patients have a more desynchronous response pattern, their d's are expected to be larger.

Finally, the last method to test for synchrony/desynchrony consisted of scoring all $\Delta 1$ and $\Delta 2$ variables in one of five categories: strong habituation, meaning that the response was less than 30 of the original response (-); habituation, meaning a response of $75-30 \%$ of the initial response $(-)$; no change, a response of $75-125 \%$ of the initial response $(0)$; sensitization, a response of $125-170 \%$ of the initial response $(+)$; and strong sensitization, more than $170 \%$ of the initial response $(t+)$. When the $\Delta$-scores of two variables fall into the same category, they are considered to have changed synchronously, but when they fall in another category, they are considered to have changed desynchronously.

\section{RESULTS}

Due to failure of equipment, for 5 subjects no FPV scores were available, for 9 subjects no EMG-paraspinal data were available and for another 9 subjects no EMGfrontal data were available. HR responses were available for only 28 out of 43 
subjects, in one subject there had been a error while recording, and the remaining subjects did not show an initial accelerative response. Therefore, no change from initial responsiveness could be calculated.

The change in mean responsiveness for all variables from the begin of the session until half-way the session and until the end of the session, is shown in figure 5.8 and 5.9. The first figure displays the changes occurring in CLBP patients, and the second figure those of controls. It is clear form the figures that the various variables do not change in the same way after stimulus repetition. Most variables seem to change in a desynchronous way. Nevertheless, it was tested whether there were any differences in this respect between CLBP patients and controls.

\section{patients}

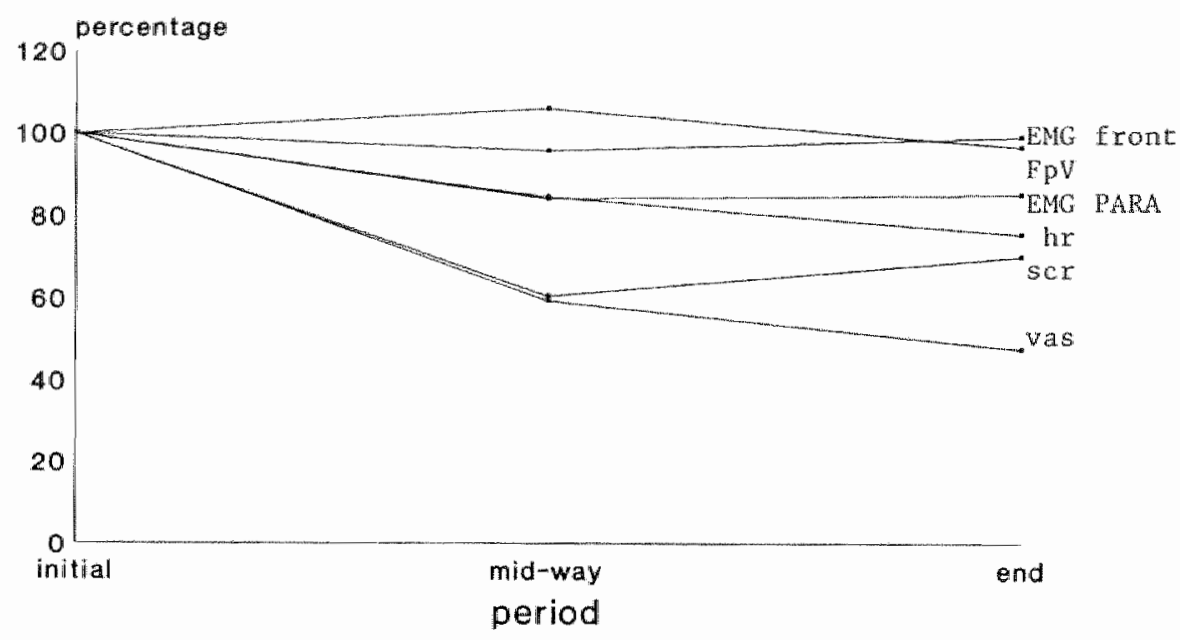

figure 5.8: Habituation curve for the various variables in CLBP patients

First, correlations between the degree of change of the various variables were computed. Fifteen correlations can be computed between all $\Delta 1$ variables in each group. Using the bonferonni correction and allowing an overall type II error of $10 \%$, the significance level was set to $0.10 / 15=0.0067$. For the $\Delta$ l variables, none of the correlations reached significance in either group. All correlations were low, or even negative, indicating that the ordering of subjects on the magnitude and direction of change of one variable was unrelated to their ordering on the magnitude and direction of change of another variable. The same was true for the $\Delta 2$ variables. Only one of the 15 correlations reached significance in the patient group: there was a correlation of .68 between the change on the HR response and the change on the FPV response.

The second method to measure synchrony/desynchrony did neither indicate a difference between the groups. Difference scores between each combination of $\Delta 1$ and $\Delta 2$ variables were calculated for patients and controls separately. The difference 
in percentage change from the initial response was large for all combinations of variables in both groups. Thus, these analyses indicate that indeed there was no synchronous change between the cognitive and physiological response system ${ }_{4}$ or even within the physiological response system itself, in both controls and CLBP patients.

\section{controls}

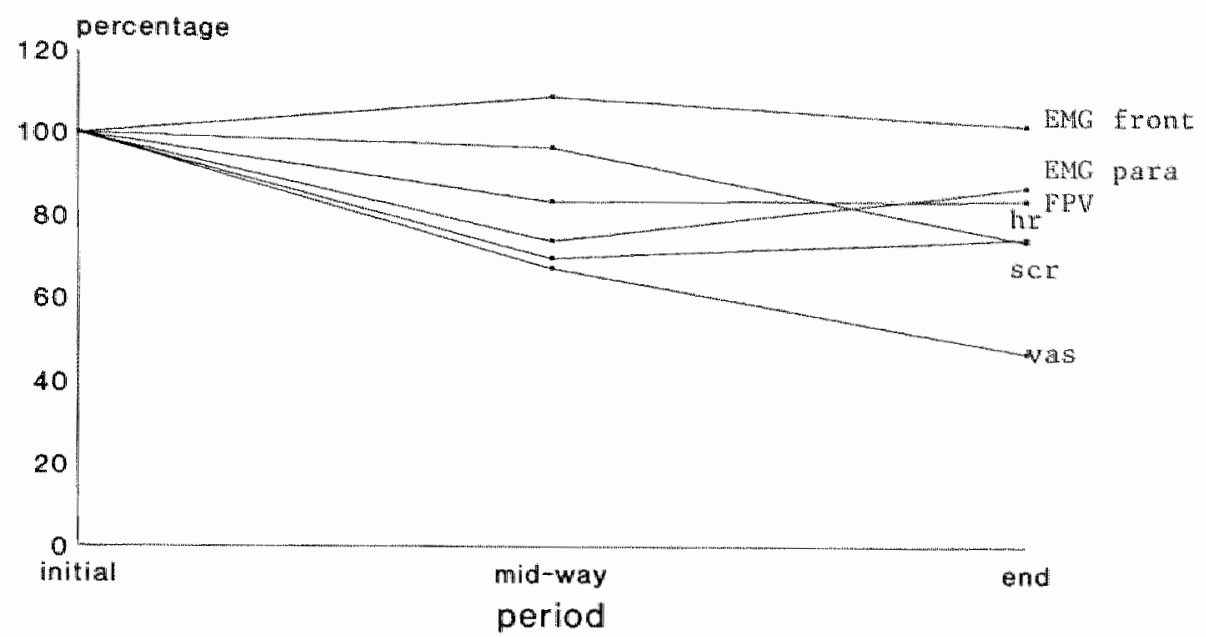

figure 5.9: Habituation curves for the various variables in controls

Even without correction for multiple comparisons, there were no significant differences at the 5\% level between the groups on any of the difference scores. This is illustrated in table 5.12 for the comparisons between the subjective pain score and all physiological parameters. The comparisons between the physiological variables themselves yielded the same picture.

Although the two methods discussed above had not yielded differences between the groups, one last method of analysis was tried, which did not depend on mean scores within groups. Averaging sometimes leads to loss of important information. Therefore, for every subject it was assessed whether two variables changed synchronously or not. Two variables were said to change synchronously when their percentage of change fell into the same category (e.g. "strong habituation" or "no habituation", see above). The results can be seen in table 5.13. This table shows for each variable in which category it falls. Only the change from initial level to the 20th stimulus is displayed. The results of the analyses of changes from the beginning until half-way were quite comparable. The number of times a response change fell into the same category for two variables was roughly the same. 


\begin{tabular}{|c|c|c|c|c|c|}
\hline & CLBP pat & & controls & & \\
\hline variable & mean & $n$ & mean & $n$ & $\mathrm{p}$ \\
\hline$\triangle V A S 1-\triangle S C R 1$ & $\begin{array}{l}45.0 \\
(28.8)\end{array}$ & 22 & $\begin{array}{l}33.9 \\
(27.0)\end{array}$ & 21 & .201 \\
\hline$\triangle V A S I-\triangle F P V I$ & $\begin{array}{l}58.1 \\
(36.1)\end{array}$ & 20 & $\begin{array}{l}52.0 \\
(52.0)\end{array}$ & 19 & .673 \\
\hline$\triangle V A S 1 \cdot \triangle H R 1$ & $\begin{array}{l}121.8 \\
(182.9)\end{array}$ & 16 & $\begin{array}{l}100.9 \\
(90.7)\end{array}$ & 12 & .694 \\
\hline$\triangle V A S 1-\triangle E M G$ front1 & $\begin{array}{l}146.8 \\
(164.5)\end{array}$ & 19 & $\begin{array}{l}92.0 \\
(134.3)\end{array}$ & 14 & .412 \\
\hline$\triangle$ VAS1- $\triangle E$ EGparal & $\begin{array}{r}81.5 \\
(73.6)\end{array}$ & 16 & $\begin{array}{l}121.4 \\
\mid 322.21\end{array}$ & 18 & 616 \\
\hline$\triangle V A S 2-\triangle S C R 2$ & $\begin{array}{c}49.4 \\
40.4)\end{array}$ & 22 & $\begin{array}{l}46.7 \\
\{40.9\}\end{array}$ & 21 & .825 \\
\hline$\triangle V A S 2-\triangle F P V 2$ & $\begin{array}{c}58.4 \\
(41.2)\end{array}$ & 19 & $\begin{array}{l}64.4 \\
(57.0)\end{array}$ & 19 & .712 \\
\hline$\overline{\triangle V A S 2}-\triangle H R 2$ & $\begin{array}{c}151.4 \\
\{238.6\}\end{array}$ & 16 & $\begin{array}{c}90.3 \\
(224.5)\end{array}$ & 12 & .498 \\
\hline$\triangle$ VAS2 - $\triangle E M G$ front2 & $\begin{array}{c}86.4 \\
(59.0)\end{array}$ & 19 & $\begin{array}{l}65.8 \\
178.31\end{array}$ & 14 & .441 \\
\hline 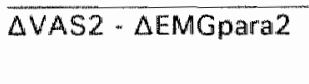 & $\begin{array}{c}81.7 \\
(82.3)\end{array}$ & 16 & $\begin{array}{l}134.4 \\
\mid 367.31\end{array}$ & 18 & .435 \\
\hline
\end{tabular}

Table 5.12: The upper part shows the difference between the change in VAS ratings from the initial assessment period until the 10th stimulus $\{\triangle V A S 1\}$ and the changes in physiological reactivity from beginning until thalf-way the session, for patients and controls separately. The lower part shows the same for the changes in VAS ratings $\triangle$ VAS2I and the changes in all physiological variables from initial rating until the end of the session.

It is apparent from the table that the changes in responsiveness are rather different for the various variables. The predominant changes for VAS ratings and SCR's were in the direction of habituation. For FPV responses, there was usually no change, whereas HR and EMG responses could change in either direction for different subjects.

In addition, it is apparent that in both groups the change of two variables was often desynchronous. The number of times that two variables fall into the same category does not exceed chance level. As discussed above, these same results were obtained for the comparisons of the changes from initial level to half-way the session. 


\section{$\triangle V A S \quad \triangle S C R \quad \triangle H R \quad \triangle F P V$ Afront $\triangle p a r a$}

\begin{tabular}{|c|c|c|c|c|c|c|}
\hline \multicolumn{7}{|l|}{ patients } \\
\hline & $-\infty$ & - & & - & 4 & + \\
\hline & - & 0 & + & 0 & 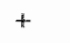 & + \\
\hline & - & $\cdots$ & $\cdots$ & - & & $\ldots$ \\
\hline & 0 & 0 & & 0 & 0 & + \\
\hline & -- & 0 & + & & & \\
\hline & - & $-\infty$ & "- & 0 & -- & \\
\hline & 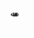 & $\cdots$ & -- & 0 & + & \\
\hline & - & 0 & & & - & \\
\hline & - & -- & $+t$ & + & 0 & \\
\hline & $\cdots$ & - & + & 0 & ++ & - \\
\hline & -- & 0 & ars & 0 & - & + \\
\hline & $\cdots$ & - & & 0 & + & $\cdots$ \\
\hline & 0 & - & & 0 & & - \\
\hline & $"$ & - & -- & 0 & - & \\
\hline & 0 & - & 0 & & 0 & + \\
\hline & 0 & $\cdots$ & $\ldots$ & 0 & $=$ & 0 \\
\hline & 0 & $=$ & $\cdots$ & 0 & + & H \\
\hline & - & - & + & at" + & - & $\cdots$ \\
\hline & - & $\ldots$ & $+t$ & 0 & 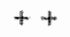 & 0 \\
\hline & $\cdots$ & $t+$ & $\ldots$ & 0 & 0 & ++ \\
\hline & -- & -- & & 0 & . & + \\
\hline
\end{tabular}

\section{controls}

\begin{tabular}{|c|c|c|c|c|c|}
\hline 0 & - & & - & - & 0 \\
\hline- & - & & & $\ldots$ & +4 \\
\hline.- & - & ++ & 0 & - & - \\
\hline - & - & - & 0 & * & 0 \\
\hline$\checkmark$ & 0 & -- & 0 & 0 & - \\
\hline -. & - & 0 & - & $\#$ & +4 \\
\hline - & - & & $+t$ & 0 & - \\
\hline - & 0 & & 0 & $\ldots$ & $\ldots$ \\
\hline - & 0 & 0 & & & 0 \\
\hline$-\infty$ & - & .. & - & & -- \\
\hline 0 & 0 & - & + & & +4 \\
\hline-- & $\cdots$ & & 0 & & \\
\hline$\because$ & + & - & +4 & $+n$ & $=$ \\
\hline- & $\cdots$ & & 0 & 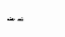 & \\
\hline 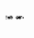 & + & - & 0 & & \\
\hline . & 0 & & 0 & - & . \\
\hline 0 & 0 & & 0 & 0 & - \\
\hline - & - & - & - & & +4 \\
\hline . & -. & 0 & 0 & & $m$ \\
\hline$\ldots$ & 0 & -- & * & m & + \\
\hline 0 & + & & - & +4 & $\cdots$ \\
\hline
\end{tabular}

Table 5.13: change scores for all variables from the beginning to the ond of the stimulation period. - means strong habituation, wabituation, 0 no changle, + sensitization and + + strong sensitization. 


\section{GENERAL DISCUSSION}

Two separate studies reported in this paper have shown that there is little evidence for an increased discordant or desynchronous response pattem in CLBP patients in an experimental pain situation. In the first part of this paper the discordance between subjective pain ratings of electrical stimuli and the SCR's to these stimuli was assessed. It was demonstrated that in both the patient group and the control group some subjects displayed concordant response patterns, while others had more discordant patterns. The fact that there was no close concordance between the VASscores and SCR in the control group came as no surprise because autonomic responses are non-specific indicators of nociceptive activation. They can be elicited and influenced by many different stimuli. Therefore, a certain degree of discordance was expected in all subjects. Nevertheless, it is still possible to test whether the degree of discordance is relatively more prominent in CLBP patients than in controls. Our analysis showed that this was not the case. Moreover, the results also showed that in both groups there was a strong relationship between actual stimulus intensity and perceived pain intensity. Thus, CLBP patients seem to be equally capable of perceiving nociceptive sensations accurately as pain-free controls. Patients did not show a pattern of over-reporting or undifferentiated responsiveness due to response tendencies in this situation.

In the second part of this paper, it was found that the responses of all subjects changed in a predominantly desynchronous way. Tested in three different manners, all analyses indicated that in this particular circumstance, desynchrony between subjective and physiological variables and between the different physiological variables themselves, was the rule rather than the exception. This confirms earlier research findings. Several experiments have demonstrated that the degree and speed of habituation differs for the subjective and physiological pain variables, and also between the various physiological pain variables (see section 4.3).

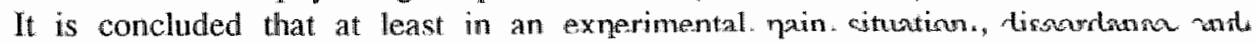
desynchrony cannot be considered typical of chronic pain patients. However, in contrast to the obvious advantage of experimental tests, a major disadvantage is its restricted generalizability to the clinical situation. In the original formulation of Gannon and Haynes, it was supposed that there would be discordance between the physiological reactions responsible for the particular complaint and the cognitive awareness of these reactions. In the present study, discordance of pain responses to stimuli unrelated to the clinical pain complaint were measured. When the lack of awareness of physical reactions is a general deficit occurring in CLBP patients, experimental studies like the present one are appropriate to demonstrate this. Howewer, when the deficit is more specific, related to the pain-relevant response system, such experiments will not suffice. Similarly, when cognitive-physiological discordance is the result of differential leaming processes and social contingencies on the cognitive and physiological response system, this may of may not generalize to the laboratory situation. It can generalize to the laboratory situation when over- 
reporting of pain has become a habitual response pattern. In this case, absence of habituation of subjective pain responses in combination with a normal habituation of physiological responses can be hypothesized. This was not found in the present study. Subjective pain responses declined more rapidly than physiological responses, in controls as well as CLBP patients. But considering the fact that social contingencies will be different in a patient's own social surrounding than in a laboratory situation, over-responding not necessarily generalizes to an experimental situation. Whereas the patient's spouses may be potent reinforcers of clinical pain responses, the experimenter may be more neutral in this respect as regards experimental pain responses.

In conclusion, cognitive-physiological discordance is not a robust feature of CLBP patients, generalizing to all situations. However, the more clinically specific form of cognitive-physiological discordance is still open for testing. 


\title{
5.5 A COMPARISON OF TWO-POINT DISCRIMINATION THRESHOLD OF TACTUAL, NON-PAINFUL STIMULI BETWEEN CIIRONIC LOW BACK PAIN PATIENTS AND CONTROLS $^{1}$
}

\begin{abstract}
Differences in pain sensitivity beween chronic pain patients and healthy controls have been reported. Seltzer and Seltzer extended this line of research in studying the sensitivity to non-painful stimuli. They reported that the 2-point discrimination threshold of chronic pain patients was higher than that of control subjects. However, the study of Seltzer and Seltzer suffered from several methodological shortcomings. Therefore, in the present study we tried to replicate the findings in a group of chronic low back pain patients using a design that was believed to be methodologically stronger. Replication failed: no evidence was found for the hypothesis that chronic pain patients are less sensitive to non-painful stimuli. Further studies on various defined types of acute and chronic pain patients are required.
\end{abstract}

\section{INTRODUCTION}

Several studies have shown chronic pain patients to be less sensitive to acute experimental nociceptive stimuli. Both a lower pain perception threshold and a lesser discrimination between pain stimuli of different intensities have been found (Cohen et al, 1983; Naliboff et al, 1981; Yang et al., 1985). Usually this lesser sensitivity is interpreted to be a result of chronic pain (Lipman et al., 1987; Yang et al., 1985), and several psychological and physiological mechanisms have been proposed to account for it (LeBars \& Willer, 1988; Naliboff et al., 1981; Yang et al., 1985). A logical extension of this line of research seems to be the assessment of the sensitivity of chronic pain patients to non-nociceptive stimuli. This was attempted by Seltzer \& Seltzer (1986), who compared the 2-point discrimination threshold for tactual stimuli applied to the forearm in a group of chronic pain patients and a control group. The study showed that chronic pain patients had a higher 2 -point threshold, and thus were less sensitive to non-noxious tactual stimulation than control subjects.

However, we considered that the procedure Seltzer \& Seltzer used to apply the stimuli

\footnotetext{
"Reprinted from: Peters, M.L. \& Schmidt, A.J.M., A Comparison of two-point discrimination threshold of tactual, non-painful stimuli between chronic low back pain patients and controls, Pain, $44(1991) 57.60$
} 
was not very reliable. The flaws in their design will be addressed in the Discussion. Because altered sensitivity as a consequence of pain is a theoretically interesting issue, we decided to try to replicate the findings using a more reliable design.

\section{METHOD}

\section{Subjects}

Forty subjects participated in the study: 20 chronic low back pain (CLBP) patients and 20 control subjects, matched for age and gender. Each groups consisted of 10 men and 10 women. The mean age was 43.6 years (range 21-55 years) for the patient group and 43.6 years (range 20-60 years) for the control group.CLBP patients were recruited by means of an advertisement in a local newspaper. The selection criteria for CLBP patients were: (1) a history of at least one year of low back pain, (2) no medically diagnosed illness or pathology, (3) the constant presence of pain. Partners of the patients, relatives or acquaintances served as control subjects. The control subjects were screened for the absence of pain of any kind. All subjects completed a questionnaire on previous illnesses, daily activities and present health. In addition, CLBP patients completed a questionnaire about their back pain. Both questionnaires were screened by a general practitioner before subjects were admitted to the study.

The mean duration of back pain for the patient group was 8.6 years (range 1- 30 years). All patients had regularly visited the general practitioner for their back pain, and most of them (14 subjects) were also examined by a specialist (neurologist or orthopaedist). This had not resulted in a clear diagnosis for any of the subjects, nor in an effective treatment for pain relief. It can be assumed that this patient population consisted mainly of patients with pain of the so-called idliopathic type, as defined by Williams \& Spitzer (1982).

All subjects had been instructed not to take any analgesics on the day of testing. Subjects were paid after participation.

\section{Apparatus}

A compass was used to deliver the stimuli to the subjects. The distance between the legs of this compass could vary between 0 and $200 \mathrm{~mm}$, with an accuracy of $1 \mathrm{~mm}$. The distance was measured along a fixed ruler.

\section{Procedure}

During a 1.5 hour session, subjects participated in several tests. Two 2-point discrimination tests were interposed between tests of pain sensitivity (in which short duration electrical stimuli were delivered to the right and left ankle). The first 2-point 
discrimination test took place approximately halfway through the session, while the second was carried out a little later. Details of the whole study will be published later.

During the 2-point discrimination test, subjects wore black goggles. The right arm was placed on the table with the palm of the hand in an upward position. Following the procedure of Seltzer and Seltzer, the stimuli were applied to the lateral side of the ventral forearm. Some standardization of the location of stimulation was achieved by measuring the distance from wrist to elbow. Halfway allong, the skin was marked above the radus (identified by palpation) by a small dot. All stimuli were centred around this dot or, in cases where the skin was touched by only one leg of the compass, at the dot itself.

We chose a different procedure to deliver the stimuli than Seltzer and Seltzer did. In their study, ascending and descending series of stimuli were presented to the subject in order to assess discrimination threshold. Although they frequently introduced a "catchtrial", in which the subject"s arm was deliberately touched by only one point of the instrument, in our opinion response bias was not avoided allogether with this procedure. Especially because after the first report of " 1 point" in the descending series and " 2 points" in the ascending series, stimulation was stopped immediately. Inaccuracies of reports could thereby greatly infuence the results.

We therefore decided to present stimuli at small and large intervals in random order. Based on the results of Seltzer and Seltzer we selected stimulation distances of 10-50 $\mathrm{mm}$. However, in a pilot study it was shown that for some subjects this interval was too small, because they could not identify a distance of $50 \mathrm{~mm}$ as 2 points. Therefore we decided to test the global sensitivity of a subject first, by presenting 6 pretest trials of 1 , $50,57,1,54$ and $48 \mathrm{~mm}$. If the subject could not correctly identify 3 of the 4 large intervals, a higher range of stimulation was chosen: 40 to $80 \mathrm{~mm}$.

The discrimination test was presented twice to all subjects. Both tests consisted of 50 trials, 41 test trials ( 1 presentation of every mm between 10 and $50 \mathrm{~mm}$ in the low range, and between 40 and $80 \mathrm{~mm}$ in the high range) and 9 "catch-trials" (8 presentations of 1 point, and once a stimulus of very large distance: $60 \mathrm{~mm}$ in the low range and 90 $\mathrm{mm}$ in the high range). The stimuli were presented in a fixed random order. This order was the same for the high and low ranges; $30 \mathrm{~mm}$ was added to the stimuli of the low range to obtain the high range. The inter-stimulus interval was about 5 seconds. After every 10 stimuli, the experimenter firmly stroke the subject's arm a few times to prevent interference from any residual sensations from previous stimuli. Subjects had to choose between 1 and 2 after every trial and, when in doubt, they were forced to make a choice. The same procedure was followed the second time, where the same stimulation distances were presented backwards.

\section{Scoring}

Scoring was done in the following way. A subject scored every distance twice (once in every series), giving a total of 82 scores. These scores were arranged along an ascending distance axis. The pilot study had shown that subjects were very consistent in their scores at both extremes, scoring only l's for the small distances and only 2's for the large distances. However, somewhere along the axis, there is an area in which scores 
become inaccurate and 1 "s and 2's are alternated. The "true" 2-point discrimination threshold must be somewhere within this area. Theoretically, all distances smaller than this threshold should be perceived as 1 point, while all larger distances should be perceived as two points. This means that all 2 's on the left side of the threshold point, and all I"s on the right side, are considered to be incorrect responses. The "true" thresthold is determined in such a way that there is an equal amount of incorrect responses on both sides of this point.

The "catch trials" were not considered in the actual scoring procedure, because they were meant as a control for the reliability of the method, as well as a measure of the ability of the subject to respond accurately.

\section{RESULTS}

The results of 4 subjects ( 3 controls and 1 patient) had to be omitted because their scores were unreliable: either they scored very inconsistently, which did not allow us to determine the threshold, or the scores on the "catch-trials" showed that the answers were not reliable ( 1 subject). The remaining 36 subjects received a total of 648 "catch trials", of which 23 were scored incorrectly $(3.5 \%)$. Ninety-five percent of the times the responses were accurate in the control group, $97 \%$ in the CLBP group. Both groups have a slightly better accuracy compared to that reported by Seltzer and Seltzer 90 and $93 \%$ , respectively).

In both groups, individual thresholds were averaged to obtain a mean discrimination threshold per group. The mean discrimination threshold of the control group was 41.8 $\mathrm{mm}$ (sd. $13.8 \mathrm{~mm}$ ), that of the CLBP patients was $45.5 \mathrm{~mm}$ (sd. $14.0 \mathrm{~mm}$ ). A t-test for unpaired observations showed that this difference was not statistically significant ( $t=-$ $0.79, \mathrm{df}=34, \mathrm{p}=0.44$ ). To test for any sex differences, a $2 \times 2$ ANOVA was applied to the data. Again, there was no group effect, no sex effect and no sex $x$ group interaction.

Finally, a non-parametric procedure was also tried (Mann-Whitney signed rank test). Neither did this indicated a difference between the groups.

\section{DISCUSSION}

In contrast to the results obtained by Seltzer and Seltzer, we found no evidence of an increased discrimination threshold for CLBP patients. In addition, the threshold scores we obtained were higher than the ones which were previously reported. It should be seriously considered whether the results obtained in the Seltzer and Seltzer study could not have been due to methodological shortcomings in their study. Our objections to their methodology will be addressed below.

Firstly, our pilot study indicated that slight differences in the stimulation location 
could considerably influence the threshold. Seltzer and Seltzer do not mention whether the location of stimulation was adequately controlled between subjects. If not, bias could have been introduced. Differences in location of stimulation between the two studies can possibly also explain why we obtained higher mean thresholds for both groups.

Secondly, a major problem in the sudy of Seltzer and Seltzer is the way in which the stimuli were delivered. Our results show that subjects do not score very consistently in trials which present stimuli centered around the discrimination threshold, despite the fact that they are very accurate on "catch trials". Considering the first transition from 1 to 2 , or from 2 to 1, as an indication of the threshold as Seltzer and Seltzer did, is not a very reliable procedure. Moreover, applying ascending and descending stimulus series induced expectation effects in subjects.

Thirdly, the descending series in the Seltzer and Seltzer study seems to have started with distances which were too small. The largest distance which was presented was 46 $\mathrm{mm}$. We obtained several thresholds above $50 \mathrm{~mm}$. And so must Seltzer and Seltzer have, considering that their mean threshold for patients was $40.3 \mathrm{~mm}$, with a sd. of $\mathbb{1 5}$ $\mathrm{mm}$ ! A simple calculation of probability (under the assumption of a normal distribution of scores) tells us that approximately 6 or 7 subjects in their patient group (35\%) cannot possibly have reported a threshold in the descending series.

There remain some other differences between the 2 studies. First, the instruments used were different. Although this might have caused differences in the absolute value of the threshold, it is thard to see why this would have caused a significant difference between patients and controls with one instrument while not with another. Moreover, the reliability of our apparatus does not seem to be less than the Seltzer and Seltzer device: from the 356 "catch trials" they performed, more than $8 \%$ were scored incorrectly compared to $3.5 \%$ of our 648 "catch trials". Another difference between the studies is the selection of patients. The Seltzer and Seltzer study used patients with different pain syndromes who were referred to a pain clinic, while we used CLBP patients only. Seltzer and Seltzer do not report whether their patient population included patients with organic diseases as well. A neurological disease can decrease tactual sensitivity. Such patients were explicitly excluded in our study.

Finally, we must acknowledge that the different means of patient selection could have contributed to the diverging results of the two studies in yet another way. Seltzer and Seltzer suggested 2 mechanisms to explain their finding of reduced sensitivity in chronic pain patients: (1) anxiety due to exposure to aversive events and (2) learned helplessness resulting from lack of control over these events. Patients referred to a pain clinic might be more anxious and have a more helpless attitude than responders to an advertisement. Considering the methodological problems listed above, the results of Seltzer and Seltzer should be viewed with caution. 


\subsection{THE INFLUENCE OF A COUNTERIRRITATION STIMULUS ON PAIN PERCEPTION AND PAIN DISCRIMINATION}

\section{INTRODUCTION}

Counterirritation is the phenomenon that one pain decreases the perception of another pain. This phenomenon has been demonstrated experimentally, by the application of wo simultaneous pain stimuli. Usually, the effect of a tonic pain stimulus on the perception of a short phasic pain stimulus has been assessed (Pertovaara et al., 1982; Willer et al., 1984; Chen et al., 1985; Chen \& Treede, 1985; Talbot et al., 1987; Pantaleo et. al., 1988). Clinical pain was also found to have a counterirritation effect (Willer et al., 1985; Calvino \& Le Bars, 1986; Willer et al., 1987) and it has been suggested that the increased pain threshold found in chronic pain patients is possibly explained by counterirritation (Le Bars \& Willer, 1988). Other studies have demonstrated that chronic pain patients have a decreased capacity to discriminate between different intensities of painful stimuli (Naliboff et al., 1981; Yang et al., 1985; Malow et al. 1980). Can counterirritation also explain such a decreased pain discrimination capacity?

The proposed neurophysiological basis of counterirritation is the mechanism which has been called diffuse noxious inhibitory controls (DNIC). This mechanism was formulated on the basis of findings from animal research. Characteristic of DNIC is the diffuse nature of the inhibitory process: noxious stimuli on any part of the body can induce inhibition of heterotopic nociceptive spinal and medullary neurons. Supraspinal connections are essential in the activation of DNIC. Another important feature of this mechanism is that especially convergent neurons (neurons which are activated by nociceptive and non-nociceptive input) have been implicated to play a role in DNIC (Le Bars et al., 1979 and 1979b). The role of nociceptive specific neurons is less clear. The specific function which convergent neurons have in pain perception is controversial. It has been suggested that convergent neurons may be necessary for the detection of small changes in nociceptive intensity (Bushnell et al., 1984). Considering this, Talbot et al. (1989) have argued that the inhibition of these neurons by DNIC should lead to impaired discrimination between different intensities of a noxious stimulus. This hypothesis was tested in an experiment in which the reaction time that a subject needed to detect a change in intensity of a gradually increasing painful heat stimulus was measured in two conditions: with and without the simultaneous application of the cold pressor test. Talbot et al. found that in combination with the cold pain, subjects showed an increased latency to detect the increment.

Thus, counterirritation can impair experimental pain discrimination. This may indicate that the decreased pain discrimination which has been found in chronic pain patients is possibly also caused by counterirritation.

The two purposes of the present study were (a) to replicate the finding of Talbot et al. that a counterirritation stimulus can decrease discrimination capacity, and (b) to 
develop a reliable procedure to measure this discrimination capacity which can be used in the study of chronic pain patients. For practical reasons we used a different procedure than Talbot. Mechanical pressure was used as the counterimitation stimulus. For the detection task, painful electrical stimuli were used. Our discrimination task consisted of a forced choice test in which pairs of stimuli were presented and subjects had to decide which of the two stimuli was the stronger one. Thus procedure has the advantage that response tendencies or expectancy effects are barned.

In addition, absolute detection threshold, pain perception threshold and pain tolerance for the electrical shocks were measured.

Two hypothesis were formulated:

1. The presence of the pressure pain stimulus raises the pain perception threshold and maximal pain tolerance for electrical pain stimuli

2. The presence of the pressure pain stimulus decreases the ability to discriminate between noxious electrical stimuli of different intensity

Exploratory, the effect of the pressure pain stimulus on detection threshold was assessed. DNIC is said to influences the perception of noxious stimuli to a greater extent than the perception of non-noxious stimuli. However, convergent neurons are also activated by input from A-alpha (non-noxious) fibers, and this activity is suppressed by DNIC to some extent at least. Therefore, a suppressive effect of a counterirritation stimulus on DT could be possible.

\section{METHOD}

\section{Subjects}

Twenty healthy, pain-free volunteers ( 9 men, 11 women) participated in the experiment. They were all university students. Mean age was 22.2 years with a range of 19 to 29 years. Participants received a small financial reward.

\section{Apparatus}

A modified Forgione-Barber mechanical pressure stimulator (Peters et al., 1989) was used to deliver a tonic pressure pain stimulus. In short, this apparatus consists of a lever with an attached plexiglass wedge which is placed on the subject's finger. By sliding a weight along the lever, the pressure on the finger can be varied. In the counterirritation condition a total pressure of 1200 to $1900 \mathrm{grams}$ was exerted. This induced a dull aching sensation. In the control condition, the weight was removed and a slight pressure from the lever itsell was exerted on the finger.

The electrical current generator was a Siemens neuroton 627 . Stimuli of 4 seconds duration with a $20-\mathrm{Hz}$ frequency were used in this study. Two Beckman $\mathrm{Ag} / \mathrm{AgCl}$ electrodes Filled with Hewlett-Packard redux creme were attached to the left lateral side 
of the leg just above the ankle. Anode and cathode were always in the same position.

\section{Procedure and design}

Each subject participated in two conditions: the counterirritation condition (CI) in which two simultaneous pain stimuli were presented and the control condition (CON) in which only one pain stimulus was presented. The order of the two conditions was varied between subjects. A 10 minutes interval separated the two conditions, in order to prevent after effects of counterirritation influencing the control condition.

The experiment took place in a non-distracting surrounding. Subject were seated at a table with the pressure stimulator and a clock in front of them. The electrical current stimulator was positioned out of sight of the subject and was operated by one of the experimenters. A second experimenter sat in front of the subject and operated the pressure stimulator.

\section{Conditions}

In the CI condition, an appropriate weight for the Forgione-Barber stimulator was established for each subject. This was the weight at which pain was elicited in approximately 1 minute and could subsequently be tolerated for 3 minutes or more. The wedge was placed on the lateral side of the second phalanx of the thumb. The two thumbs were used alternatingly, to prevent sensitization. To maximize tolerance times, every subject was told that most people could endure the pain for at least 3 to 4 minutes, and they were encouraged to look at the clock in front of them. It ustually did not take more than two or three trials to find the appropriate weight.

Following this establishment phase, assessment of pain perception and pain discrimination for electrical shocks took place. In the counterirritation condition, the pressure stimulus was applied to the thumb at the same time. Only after pain was reported, the electrical stimuli were delivered by the second experimenter. Subject were instructed not to pay attention to the pain in their thumb, but to concentrate on the electrical stimuli on the leg.

In the CON condition the weight was removed from the lever and only a light pressure was exerted on the thumb. This did not produce a painful sensation in any of the subjects (checked afterwards).

In both conditions, first the pain perception task was presented followed by the pain discrimination task. Between the two task, a short interval took place.

\section{Dependent variables}

A series of electrical stimuli of increasing intensity was given. Stimulation started at $0.1 \mathrm{~mA}$ and initially increased in steps of $0.1 \mathrm{~mA}$ (up to $1 . \mathrm{mA}$ ) and later in steps of 0.2 $\mathrm{mA}$. The maximum current intensity to be delivered was set at $8.0 \mathrm{~mA}$. Three perception 
levels were recorded:

- detection threshold (DT): when the electrical stimulus was felt for the first time

- pain perception threshold (PPT): when the electrical stimulus became painful for the first time

- maximal pain tolerance (MPT): when the subject did not want to continue the increasing series of electrical stimuli. Three subject reached the $8.0 \mathrm{~mA}$ ceiling (in both conditions).

In the pain discrimination task, the dependent variable was the number of incorrect choices in a forced-choice task. Eight pairs of stimuli were delivered. The two stimuli within one pair always differed $0.2 \mathrm{~mA}$. Two kinds of pairs were presented: a high intensity pair (0.1 $\mathrm{mA}$ and $0.3 \mathrm{~mA}$ below an individual's MPT) and a low intensity pair (0.1 mA and $0.3 \mathrm{~mA}$ above an individual's PPT). Both pairs were presented four times in a prefixed order, with the stronger stimulus being the first half of the time. After each pair, the subject had to decide which of the two stimuli was the stronger one. When in doubt, subjects were urged to make a choice.

\section{RESULTS}

$\mathrm{Cl}$ CON sign.

\begin{tabular}{|c|c|c|c|c|}
\hline DT & $\begin{array}{l}\text { median } \\
\text { mean } \\
\text { (sd) }\end{array}$ & $\begin{array}{l}0.1 \\
0.13 \\
(0.06)\end{array}$ & $\begin{array}{l}0.1 \\
0.13 \\
10.041\end{array}$ & n.s. \\
\hline PPT & $\begin{array}{l}\text { mediam } \\
\text { mean } \\
(\mathrm{sid})\end{array}$ & $\begin{array}{l}1.0 \\
1.06 \\
(0.67)\end{array}$ & $\begin{array}{l}0.85 \\
1.14 \\
(0.84)\end{array}$ & n.s. \\
\hline $\mathrm{MPT}$ & $\begin{array}{l}\text { median } \\
\text { mean } \\
(\mathrm{sd})\end{array}$ & $\begin{array}{l}2.8 \\
3.2 \\
(1.48)\end{array}$ & $\begin{array}{l}2.6 \\
3.11 \\
(1.70)\end{array}$ & n.s. \\
\hline $\begin{array}{l}\text { number of } \\
\text { incorrect } \\
\text { choices }\end{array}$ & $\begin{array}{l}\text { median } \\
\text { mean } \\
\text { (sd) }\end{array}$ & $\begin{array}{l}2.0 \\
2.25 \\
1.291\end{array}$ & $\begin{array}{l}1.5 \\
1.45 \\
(1.00)\end{array}$ & $\begin{array}{l}p=0.01 \\
(1 \text {-tailed })\end{array}$ \\
\hline
\end{tabular}

Table 5.14: median, mean and standard deviation for the various variables in the $\mathrm{Cl}$ and $\mathrm{CON}$ condition. Differences of the median were tested at a significance level of $0.05 \mathrm{ln} . \mathrm{s}=$ non significant). 
The results are displayed in table 5.14. Medians, mean values and standard deviations (in parentheses) are shown. Because the scores did not conform to the normall distribution, non-parametrical tests were performed (Wilcoxon signed rank test for paired observations).

The results demonstrated that there were no differences between the conditions for either DT, PPT or MPT. However, during the CI condition, subjects made more mistakes in the discrimination test. The mean number of incorrect choices increased from 1.45 (median: 1.5 ) in the CI condition to 2.25 (median: 2.0 ) in the CON condition.

Looking at the high and low intensity pairs separately, it appears that the significant difference between the conditions is exclusively due to a difference for the low intensity pair. For this pair, the mean number of mistakes increased from 0.50 in the $C O N$ condition to 1.15 in the CI condition $(p=0.041$, Wilcoxon signed rank test). For the high intensity pair, the mean number of mistakes was the same in both conditions (resp. 1.0 in the CON condition and 1.1 in the CI condition).

There was a significant repetition effect for both PPT and MPT. Mean PPT increased from 0.89 the first time to 1.32 the second time $(p=0.016)$. MPT increased from 2.84 to $3.48(\mathrm{p}<0.01)$. There was no condition $\mathrm{x}$ repetition effect.

\section{DISCUSSION}

The most prominent finding of this study was the increased number of mistakes in the pain discrimination test in the counterirritation condition. In contrast, detection threshold for the electrical stimulus, pain perception threshold and pain tolerance remained unchanged. The decreased capacity to discriminate between noxious intensities when a counterirritation stimulus is presented agrees with the study of Talbot. It may be concluded that the discrimination deficit is not restricted to noxious heat stimuli, but applies to other modalities as well.

In the Talbot et al. study, all stimuli started at the same noxious intensity. In the present study, two levels of nociceptive intensity were used: mildly painful (near PPT) and fairly painful (near MPT). We found that only at the mildly painful level counterirritation exerted its effect. A complicating factor in interpreting this finding is that it is not certain whether all low intensity pairs were still experienced as painful during the discrimination test, because strong habituation effects were found to occur during the session. Inspection of the data at an individual level revealed that the counterirritation effect on pain discrimination was somewhat larger in subjects who probably still experienced these stimuli as painful, because they did not show habituation. The conclusion that discrimination at a mildly painful level is impaired seems warranted.

A recent study of Plaghki et al. (1990) also found counterirritation to be most effective at a mildly painful level. They assessed the effect of a cold pressor test on pain intensity ratings of short laser heat pulses of various intensities. It was found that pain ratings were significantly depressed only for stimulus intensities near threshold level. Nonnociceptive warmth stimuli and strong painful stimuli were not affected. However, they 
could not find an effect on discrimination of painful stimuli using signal detection analysis (SDT), whereas decreased discrimination for non-painful warmth stimuli was found.

In the present study, we did not control for non-specific distraction effects and therefore the alternative explanation that decreased discrimination capacity was caused by distraction cannot be excluded. We attempted to control for distraction by applying a light pressure stimulus to the thumb in the control condition. However, pain probably induces stronger distraction of attention than mere tactile stimulation. Still, there are at least two arguments in fawour of a counterirritation explanation. Firstly, there is a neurophysiological foundation for the effect of counterirritation on pain discrimination. Secondly, the results agree with those of Talbot et al. These researchers did control for the distraction effect by presenting another (non-noxious) discrimination task. They demonstrated that only the pain discrimination test was affected.

Our first hypothesis - increased PPT and MPT in the Cl condition - was not confirmed. This contradicts previous findings. One explanation could be that the assessment of PPT and MPT was unreliable. Not only this study, but also one of our previous studies with electrical pain stimuli (section 5.3) showed that the first PPT and MPT assessment is lower than subsequent assessments. The first assessment is perhaps influenced by fear and unfamiliarity of the electrical stimulus. Therefore, in future studies a more reliable method to measure PPT and MPT has to be employed.

The first objective of this study was to replicate the finding of Talbot et al. that counterirritation can affect the discrimination of painful stimuli. We were able to confirm this finding, using a different procedure. The second objective of the study was to develop a reliable procedure to measure pain discrimination. The forced-choice task we used in the present experiment seems to have been adequate for this purpose. An improvement upon the procedure would be to include an appropriate control task, to test for non-specific distraction effects. Another improvement could be to increase the number of stimulus pairs, and to use more than two intensity levels.

It is obvious that in order to measure PPT and MPT reliably another procedure is called for. The present procedure was not satisfactory. Familiarizing a subject with the electrical stimulus and repeating stimulus series several times (preferentially ascending and descending series) could be one means of improving reliability.

In our next study with chronic pain patients the above mentioned considerations will be taken into account. 


\subsection{DIFFERENCES IN PAIN PERCEPTION AND SENSORY DISCRIMINATION BETWEEN CHRONIC LOW BACK PAIN PATIENTS AND HEALTHY CONTROLS ${ }^{\prime}$}

\section{SUMMARY}

Pain perception threshold (PPT), maximal pain tolerance (MPT) and pain discrimination of CLBP patients and controls were tested. PPT was significantly higher in the patient group for two different pain stimuli (electrical and pressure pain). MPT was significantly higher in CLBP patients only for the pressure pain stimulus. There was no difference between the groups in their capacity to discriminate between painful stimuli of different intensity, as measured by a forced-choice task. It is concluded that CLBP patients have a decreased sensitivity for experimental pain. Two theories which might explain this decreased sensitivity are discussed.

\section{INTRODUCTION}

For some years, research has been directed towards the question of whether patients with various chronic pain syndromes have a different perception of experimental laboratory pain than healthy controls. The result of these studies seems to depend at least partly on such factors as the pain syndrome selected, the experimental pain stimulus used and on the pain variable measured (e.g. threshold versus tolerance) (Naliboff \& Cohen, 1989). The pain syndrome most extensively studied in relation to this question has been Chronic Low Back Pain (CLBP). For CLBP patients an increased pain perception threshold and decreased discrimination between stimuli of different intensity was found (Cohen et al., 1983; Naliboff et al., 1981; Wolskee \& Gracely, 1980; Yang et al. 1985), but also decreased pain tolerance (Brands \& Schmidt, 1987; Schmidt \& Brands, 1986).

Studies using patients with other pain syndromes have obtained less consistent results (Hazouri \& Mueller, 1950; Lipman et al., 1987; Malow et al., 1980; Merskey \& Evans, 1975; Molin et al., 1973). Moreover, the methods used and patients selected have been to diverse to allow for any definite conclusions. But also pertaining to CLBP patients conclusions should be drawn with caution because pain perception threshold and pain discrimination on the one hand and pain tolerance on the other, have been measured by means of different pain stimuli (radiant heat $v s$. cold pressor pain) and the three pain

"Reprinted fram: Peters, M.L. Schmidt, A.J.M., Differences in pain perception and sensory discrimination between chronic low back pain patients and healthy controls, Journat of Psychosomatic Research, 36 11992) 47-53. 
variables have never all been measured in the same subjects. Therefore, a study measuring pain perception threshold, pain tolerance and pain discrimination by means of the same stimulus and in the same group of patients was badly needed. The present study wanted to provide for this by measuring all three variables in CLBP patients and controls using an electrical pain stimulus. Another improvement upon previous studies was the inclusion of a second pain stimulus to test for the generalizability of the results from one pain stimulus to another. A stimulus of a completely different nature than the electrical stimulus was used, namely pressure pain. Whereas for the electrical stimulus, pain threshold and tolerance were measured by means of discrete steps of increasing and decreasing intensity, the pressure pain stimulus continuously increased in intensity and pain threshold and pain tolerance were measured in seconds. Also, the body location of application varied for the two stimuli.

To measure pain discrimination, a forced-choice discrimination task was chosen, instead of the often used sensory decision theory (SDT) procedure. The discrimination index obtained by this last procedure, relies on intensity ratings of a stimulus, and contrary to what is often suggested, can therefore be influenced by response tendencies. A forced-choice paradigm is free of these influences, and in previous studies binary decision procedures have been found to obtain better discrimination scores than the SDT method (Lloyd \& Appel, 1976).

A forced-choice auditory discrimination task was also included, to control for nonspecific discrimination deficits in CLBP patients.

\section{METHODS}

\section{subjects}

Forty-three subjects participated in the study, 20 CLBP patients (12 men, 8 women) and 23 controls ( 13 men, 10 women) of comparable age (mean age for the patient group: 42.0 yrs (range: $24-53 \mathrm{yrs}$ ) and for the control group $43.9 \mathrm{yrs}$ (range: $25-59 \mathrm{yrs}$ )).

CLBP patients were selected by means of an advertisement in a local newspaper. Inclusion criteria were: low back pain for at least 1 year, daily continuous pain, age between 20 and 60 . Exclusion criteria were: malignant diseases, cardiovascular problems, general poor health, use of analgesics, prior participation in pain research and hearing problems. Control subjects consisted of the partners, relatives or friends of the CLBP patients. Inclusion criteria for the control group were: no pain complaints, general good health and age between 20 and 60 . The exclusion criteria were the same as for the patient group. All subjects received a small financial reward after participation.

\section{apparatus}

Electrical pain stimuli were delivered by a constant current generator (Siemens 
neuroton 627). The output intensity of the current ranged from 0 to $12 \mathrm{~mA}$. Every stimulus consisted of a 4-second train containing rectangular pulses of 20 msec with 20 msec interval time. Shocks were delivered to the degreased skin of the right ankle by means of Beckmann $8 \mathrm{~mm} \mathrm{Ag/AgCl}$ electrodes, filled with Spectra 360 electrode gel.

Pressure pain stimuli were applied to the middle phalanx of the second finger by a slightly modified Forgione-Barber pressure stimulator (Forgione \& Barber, 1971). A 1.7 $\mathrm{mm}$ wide plexiglass wedge with a pressure of 1850 grams was placed on the finger, causing a gradually increasing pain. Tones of $400 \mathrm{~Hz}$ were produced by a tone generator and recorded on tape. During the experiment the subjects listened to this tape through stereo headphones.

\section{procedure}

Before the experiment, subjects completed the Spielberger trait and state anxiety questionnaire (STAI). CLBP patients, in addition, completed a back pain questionnaire. The experiment took place in a soundproof room of constant temperature. The subject was seated at a table and the shock electrodes were attached to the right ankle. First, pain perception threshold (PPT) and maximal pain tolerance (MPT) for electrical stimulation were assessed. Stimulus intensity started at $0 \mathrm{~mA}$, increased in steps of 0.1 $\mathrm{mA}$, and above $1 \mathrm{~mA}$ increased in steps of $0.2 \mathrm{~mA}$. PPT was measured in three ascending and three descending series of stimuli. MPT was measured by means of the three ascending series.

The auditory discrimination task was presented next. The subjects received taped instructions, and subsequently a simple hearing test was presented to make sure that all subjects could actually detect low intensity $400 \mathrm{~Hz}$ tones. During the discrimination task, a total of 24 pairs of $3 \mathrm{sec}$. $400 \mathrm{~Hz}$ tones were presented. The subject had to detect which of the two was the louder one. For every pair, a choice was required (forced choice task). The tones differed by either $1 \mathrm{~dB}(10 \mathrm{x}), 2 \mathrm{~dB}(10 \mathrm{x})$ or $4 \mathrm{~dB}(4 \mathrm{x})$. Mean intensity and order of the louder tone varied randomly. The intensity of all tones was kept at a level which was well audible for all subjects, but non-aversive.

Hereafter, the pain discrimination task consisting of 24 pairs of electrical stimuli, was presented. The difference in intensity between the stimuli was $0.2 \mathrm{~mA}$ for all pairs. A pilot study had previously shown that for most subjects this difference was difficult to detect, though not impossible. Which of the two stimuli was the most intense varied randomly. Three kinds of pairs were constructed, on the basis of the previously assessed PPT and MPT measures. The most intense pair was 0.1 and $0.3 \mathrm{~mA}$ below MPT $(8 \mathrm{x})$, the least intense pair 0.1 and $0.3 \mathrm{~mA}$ above PPT $(8 \mathrm{x})$, and the intermediate pair was exactly in between those pairs $(8 x)$. Thus, stimulus intensity was matched for individual sensitivity.

After this test, the shock electrodes were removed, and the PPT and MPT for the pressure pain stimulus were assessed. The wedge of the pressure stimulator was placed on the second finger of the left hand, and time until the report of first perception of pain was measured in seconds. The total amount of time the subject tolerated the stimulus was taken as MPT. 


\section{RESULTS}

\section{descriptive analysis of the CLBP patient group}

All CLBP patients reported having continuous back pain. The average pain duration had been 14 yrs (range: 2-30 yr). Most patienis had pain on both sides of the lower back, with radiation into the leg or the shoulders. Only two of the patients were currently under medical treatment for their back pain. Diagnosis were obtained from the patients themselves. Ten patients indicated to have undiagnosed back pain. Four patients said that the pain had continued after an operation for disc herniation and in one patient the pain had started after trauma (a fall). Two patients indicated a muscular origin for their pain, two indicated a structural spinal abnormality, one indicated intervertebral disc degeneration. Twelve patients still had full-time or part-time occupations, five had been declared disabled (one only for $25 \%$ ) and three patients were unemployed. Usually the pain had begun gradually, and was now described as "considerable" (10 cases) or "moderate" (7 cases). Three subjects only reported weak pain.

\section{PPT and MPT}

PPT for electrical stimuli was defined as the average of the least intensity at which a stimulus was experienced as painful during two of the ascending series, and the three descending series. The first ascending series was considered a test trial, to familiarize

\begin{tabular}{llllll}
\hline & CLBP & CONTROL & N & Z & P \\
\hline PPT electr & $2.34 \mathrm{~mA}$ & $1.81 \mathrm{~mA}$ & 43 & -1.75 & 0.040 \\
& $(1.21)$ & $(1.34)$ & & & \\
MPT electr & $3.41 \mathrm{~mA}$ & $2.67 \mathrm{~mA}$ & 43 & -1.34 & 0.180 \\
& $(1.83)$ & $(1.62)$ & & & \\
PPT pressure & $29.5 \mathrm{sec}$ & $18.9 \mathrm{sec}$ & 41 & -2.69 & 0.004 \\
& $(13.7)$ & $(14.2)$ & & & \\
MPT pressure & $85.1 \mathrm{sec}$ & $46.6 \mathrm{sec}$ & 43 & -2.49 & 0.013 \\
& $(59.1)$ & $(41.3)$ & & & \\
\hline
\end{tabular}

Table 5. 15: Mean and standard deviations for pain perception threshold and pain toterance for two independent pain tests: electrical stimuli and pressure pain. PPT is tested against the one-sided alternative, because a specific prediction was made for this variable. A specific prediction was made for MPT too, but because the results are opposite to this prediction, this variable is tested
against the two-sided alternative. 
the subject with the sensation and the procedure. MPT for electrical stimuli was defined as the average of the maximal intensity the subject was willing to accept during the second and third ascending series. PPT and MPT for pressure pain were measured in seconds.

The results are presented in table 5.15. The distribution of scores markedly differed from normality, and non-parametric statistics were used (Mann-Whitney U-test). CL.BP patients had a significantly higher PPT for both pain tests. Contrary to our expectation, MPT was also found to be higher in the patient group. This difference only reached significance for the pressure pain stimulus.

\section{Pain and tone discrimination}

There were no differences between CLBP patients and controls on the pain discrimination test. The mean number of incorrect choices was 5.4 for the CLBP patient group and 5.0 for the control group. This was not significantly different (Mann-Whithey $U$-test, $z=-0.67, p=0.26$, one-tailed). Because CLBP patients received on the average stimuli of higher intensity, also an analysis of covariance was performed, with intensity as covariate. This neither indicated a significant difference between the two groups. Separate analysis for the three intensity levels showed that there were no differences between those levels in the mean number of incorrect choices. Nor was there any difference between the groups on the three levels seperately. The results of this last analysis are shown in table 5.16.

There were no significant differences on the tone discrimination task between the groups either. The mean number of incorrect choices was 2.85 for CLBP patients and 2.48 for controls.

\begin{tabular}{llllll}
\hline & CLBP & CONTROL & $N$ & $Z$ & P \\
\hline low intensity pair & 1.7 & 1.8 & 43 & -0.15 & 0.880 \\
& $(1.8)$ & $(1.9)$ & & & \\
intermediate intensity pair & 1.8 & 1.5 & 43 & -1.18 & 0.281 \\
& $(1.1)$ & $(1.6)$ & & & \\
high intensity pair & 2.0 & 1.7 & 43 & -0.76 & 0.448 \\
& $(1.4)$ & $(1.6)$ & & &
\end{tabular}

Table 5.16: Mean and standard deviations for number of mistakes for the three intensity levels seperately. Tests are performed by means of the Mann-Whitney U-test. P-values are two-teiled. 


\section{stait/rate anxiety}

CLBP patients had a somewhat higher trait anxiety score than controls (39.8 vs. 33.8). This difference proved to be significant $(t=-1.79, p=0.041$ one-tailed). State anxiety was not significantly different (32.3 for CLBP patients and 30.5 for controls). This confirms our earlier findings for CLBP patients, in which a higher trait anxiety was also found (Peters et al. 1989).

\section{DISCUSSION}

The predominant finding of this study seems to be that CLBP patients are generally less sensitive to experimental pain stimuli than controls. This was not only apparent for pain perception threshold, for which variable it was predicted, but also for pain tolerance, contradicting previous findings. Moreover, it could be generalized from one pain stimulus to the other, at least for the two stimuli used in this study.

Two hypotheses are available to explain decreased sensitivity for experimental pain in chronic pain patients. The first is based upon the adaptation level theory (Naliboff \& Cohen, 1989; Gracely, 1984). According to this hypothesis, chronic pain patients are less inclined to label a stimulus as painful, and judge an experimental pain stimulus as relatively mill because of their extensive experience with pain. This is based on the assumption that the judgennent of any stimulus occurs in comparison with the context in which it occurs (Helson, 1964). For pain patients this context is a painful state.

The other hypothesis is based on the principle of "pain inhibits pain". This phenomenon is well established in the case of two concurrent heterotopic experimental pain stimuli (Chen et al., 1985; Pantaleo et al., 1988; Price \& McHaffie, 1988; Riley \& Levine, 1988; Roby-Brami et al., 1987; Talbot et al., 1987; Willer et al., 1984) and it has been proposed that the mechanisme of "diffuse noxious inhibitory controls" (DNIC) could form the neurophysiological basis of this (Roby-Brami et al., 1987; Willer et al., 1984; Le Bars et al., 1979b; Willer et al., 1989). Le Bars \& Willer (1988) have previously suggested that the decreased sensitivity found in chronic pain patients could also be based on activation of the DNIC mechanism.

The suggestion of a generally decreased pain sensitivity as a consequence of chronic pain seems inconsistent with previous findings of lower pain tolerance in CLBP patients. However, one can speculate that for both mechanisms proposed the maximal effect will occur when the clinical pain is more severe than the experimental pain (Naliboff \& Cohen, 1989; Le Bars et al., 1979b). As the experimental pain intensity increases to tolerance level, the depressive effect of the clinical pain might be much less or even absent. In addition, it has been shown that pain tolerance in particular is greatly influenced by psychological factors. Particularly when the test is of long duration and pain increases only very gradually, one can expect that other factors besides pain sensitivity determine endurance behaviour. This would be consistent with the finding of lower tolerance in CLBP patients on the cold pressor test (with tolerance times up to 5 
minutes, and often a steady pain state after some time) and a higher pain tolerance for chronic pain patients in the study of Lipman et al. (1987), where tolerance was reached in only 8 seconds on average. In short, whether tolerance is higher, lower or identical in chronic pain patients could be determined by the relative intensities of the clinical and experimental pains, the possible psychological differences between the groups, and the particular tolerance procedure used.

The fact that we did not find a discrimination deficit for CLBP patients contrasts with the findings of previous studies. A possible explanation might be the different procedure which was used. We prefered a forced choice discrimination task to the SDT measures employed in previous studies, because of the dependence of this latter procedure on intensity ratings. In a SDT paradigm, subjects have to rate stimuli of various intensity in several categories. When the same categorie is used for stimuli of different intensity, a subject is said to discriminate badly. However, a subject who is able to perceive a slight difference in intensity, can still feel that both stimuli qualify for the same rating categorie. Because it has been shown that chronic pain patients make more use of rating categories on the high end of the scale (called response bias by SDT), this can artificially lower their discrimination score. A forced-choice procedure on the other hand, rates the capacity to perceive slight differences directly. Thus, we feel that a forced-choice task reflects the capacity to discriminate more validly. In fact, studies who have compared SDT and forced-choice procedures have found superior discrimination scores by means of the latter procedure (Lloyd \& Appel, 1976).

In the study of Cohen et al. (1983), both the SDT parameter and a forced-choice task were used. Whereas sensitivity measured by SDT was less for CLBP patients, the forced choice task did not show a difference between the groups. Malow et al. did find a discrimination deficit for pain patients by means of a forced-choice task (Malow et al., 1980). However, in this study the subjects had to discriminate between two stimuli well below pain perception threshold. In the present study, all stimuli were above PPT.

As intensity discrimination is a complex sensory and cognitive process, and neither hypotheses directly predict a change in either direction, it remains to be determined if, and under what conditions, discrimination can be affected.

The present study can be taken as supportive for both the adaptation level theory and the DNIC hypothesis, and thus, future studies should try to find evidence favouring one of the hypotheses above the other. 


\subsection{SUPPRESSION OF THE NOCICEPTIVE FLEXION REFLEX WITI TWO DIFFERENT COUNTER-IRRITATION STIMULI}

\section{SUMMARY}

Counterirritation is the phenomenon that one pain inhibits the perception of another pain. A major contribution to the research of counterirritation has been made by studies using the nociceptive flexion reflex of the biceps femoris (RIII reflex). These studies have shown that counterirritation can depress a spinally organized reflex. Usually, hot water has been used as the counterirritation stimulus. The aim of the present study was to replicate the finding that a counterimitation stimulus can depress the RIII reflex, using two different pain stimuli (cold pressor test and tourniquet ischemic pain test). Both stimuli were shown to exert a powerful inhibition on the reflex. This inhibition was apparent in most of the subjects tested.

\section{INTRODUCTION}

Counterirritation is the phenomenon that one pain inhibits the perception of another pain. Historical applications of counterirritation for the relief of clinical pain have been reported (Wand-Tetley, 1956). More recently counterirritation has been established experimentally, usually by assessing the effect of a tonic experimental pain stimulus on the perception of a phasic experimental pain stimulus (Anderson et al., 1976; Pertovaara et al., 1982; Talbot et al., 1987). Not only subjective pain experience has been found to be affected by counterirritation, supression of physiological correlates of pain has also been found (Chen et al., 1984; Chen et al., 1985; Pantaleo et al., 1988),

An important contribution to this hield of research was made by Willer and colleagues, who demonstrated that counterirritation can depress the spinally organized nociceptive flexion reflex of the biceps femoris (Willer et al., 1984). This nociceptive reflex, also called the RIII reflex, can be elicited by noxious stimulation of the nervus suralis (Hugon, 1973). It has been demonstrated that there is a good correspondence between the reflex threshold and pain threshold (Willer, 1977). Several nociceptive stimuli have been tested by Willer et al. for their inhibitory effect upon the RIII reflex. Most often the emersion of a hand in painfully hot water was used, but cold pressor pain, ischemic pain and mechanical pressure pain have also been found to decrease the RIII reflex amplitude (Willer et al., 1984).

\footnotetext{
"Peters, M.L., van den Hout, M.A. \& Schmidt, A.J.M., Suppression of the nociceptive flexion reflex with two different counterirritation stimuli, submitted for publication.
} 
The finding that a counterirritation stimulus can depress a spinally organized reflex is of major significance. It suggests that the counterimitation effect is due to the inhibition of nociceptive activity at a spinal level, and not merely due to distraction. Therefore, this research merits being carried out in other research centers as well, in order to find independent confirmation. The aim of the present study was to find such confirmation and replicate the finding that besides painfully hot water, other pain stimuli can also suppress the RIII reflex. Two pain stimuli were used, cold pressor pain and tourniquet ischemic pain. Our interest was not only in the mean counterirritation effect of these two pain stimuli, averaged over all subjects, but also in the effect at an individual level. In how many subjects can a counterirritation effect actually be demonstrated?

\section{METHODS}

\section{Stimulation and recording of the reflex}

A Medelec MS6 was used to elicit and record the RIII reflex. This integrated system consists of two stimulus control units, an isolated constant current stimulator, an amplifier with remote pre-amplifier, a four-channel oscilloscope and a photographic recording unit. The apparatus is placed out of sight of the subject, with the exception of the small current stimulator and pre-amplifier. The two stimulus control units are connected in series, so that trains of eight rectangular $0.5 \mathrm{msec}$ pulses with an internal frequency of $200 \mathrm{~Hz}$ can be generated once every 5 seconds (i.e. repetition frequency $0.2 \mathrm{~Hz}$ ). These stimulus control units drive the constant current generator, which can deliver stimuli up to $50 \mathrm{mAmps}$, and are adjustable with an accuracy of $0.5 \mathrm{mAmp}$. Shocks are delivered to the skin above the distal receptive field of the nervus suralis by Beckman $\mathrm{Ag} / \mathrm{AgCl}$ electrodes, filled with isotonic electrode gel. The skin is prepared by careful brushing with sandpaper and subsequent cleaning with alcohol.

Recordings are made from the flexor muscle biceps femoris. Disposable electrodes (Medicotest) are attached to the prepared skin in the bend of the knee above the biceps femoris, while the reference electrode is attached above the patella. The electromyographic signal is bandpass filtered between 32 and $3200 \mathrm{~Hz}$, amplified and fed to the oscilloscope and recorded on photographic paper for further analysis.

\section{Subjects and procedure}

Eleven healthy and pain-free subjects ( 8 women, 3 men) volunteered to participate in this study. Their ages ranged between 22 and 52 years. Most were colleagues and students from our department. The subjects were seated in a reclining chair, with the legs supported. Electrodes were attached, and the subject was acquainted with the sensation that the stimulus induced. The intensity of the stimulus was increased until a stable RII reflex was obtained. Thereafter, stimulation continued at this intensity for the rest of the 
experiment. Nine subjects participated in a total of five conditions. The order of the conditions was the same for all subjects: (1) control condition (2) cold pressor test condition, (3) control condition (4) toumiquet ischemic pain condition (5) control condition. Two subject only participated in the first three conditions (the reasons for this were unrelated to the experiment; subjects had other engagements).

In the three control conditions, ten recordings of the reflex were made while subjects tried to relax. In the two counterirritation ( $\mathrm{Cl}$ ) conditions (conditions 2 and 4), a variable number of reflexes was recorded, while the counterirritation stimulus was applied simultaneously. The number of recordings in the CI conditions was maximally 20 (see below). During the cold pressor (CP) test, subjects had to emerge their hand up to their wrist into cold water of approximately $4 \mathrm{C}^{\circ}$. During the tourniquet test a blood pressure cuff was inflated around the upper arm to a pressure of $200 \mathrm{mmHg}$; meanwhile the subject had to perform light muscular work with the hand of the ischemic arm. This resulted in pain in 7 out of the 9 subjects who participated in this condition. The two subjects in whom no pain was provoked were left out of the analysis.

During both $\mathrm{CI}$ conditions, recordings of the reflex were made from the time that the beginning of pain was reported, until the time pain tolerance was reached, or until twenty recordings were obtained. With the $\mathrm{CP}$ test, pain usually developped within one minute, whereas with the tourniquet test it often took several minutes before pain started. In all conditions, subjects sat with their eyes closed during the recording period. Between the $\mathrm{Cl}$ conditions and the control conditions, there was an interval of several minutes, to allow after-effects to fade out.

The duration of the entire session was approximately 45 to 60 minutes.

\section{Scoring and analysis}

The magnitude of the RIII reflex was scored from the photographic recordings by way of a digitizer. The contours of the reflex appearing within a time window of 75 to 150 msec following the end of the electrical stimulus were followed by a digitizer pen and fed into a computer. All traces were displayed on the screen and visually checked for their resemblance to the original reflex contour (for an example of the digitized signal, see figure 5.10 ). The total line length of every contour was calculated and taken as the indicator of reflex magnitude. To increase reliability, all contours were fed into the computer twice, and when the calculated line length of these two readings differed substantially, two new digitizations were performed. For every condition, ten reflexes were analyzed. When more than ten recordings were available for a $\mathrm{Cl}$ condition, only the last ten reflexes were scored.

The mean reflex magnitude in every condition was calculated for each subject. Comparisons between the reflex magnitude in the various conditions were made in percentage scores. To establish the effect of each of the two counterirritation stimuli seperately, two analyses were performed. The first analysis compared the first control condition, the $\mathrm{CP}$ condition and the second control condition, while the second analysis compared the second control condition, the tourniquet condition and the third control condition. In both analyses, the reflex magnitude of the control condition prior to the CI 
condition was used as reference, and the magnitudes of the two subsequent conditions were expressed as a percentage of this magnitude. For subjects who had participated in all five conditions, a third analysis on all conditions combined was performed.

Statistical analyses were performed by means of non-parametrical tests, a Friedman two-way anova when three or more conditions were compared, and a Wilcoxon signedrank test (for dependent samples) for pairwise comparisons of conditions. All reported p-values are two-tailed.

$A$

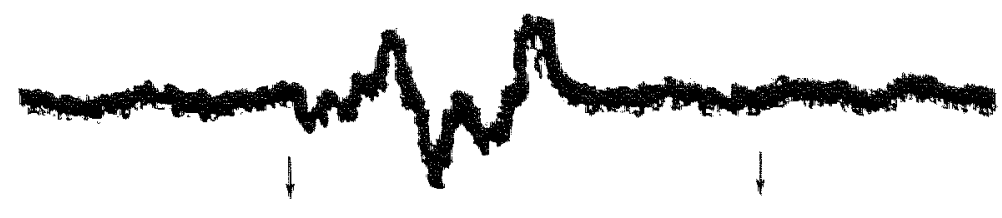

$B$

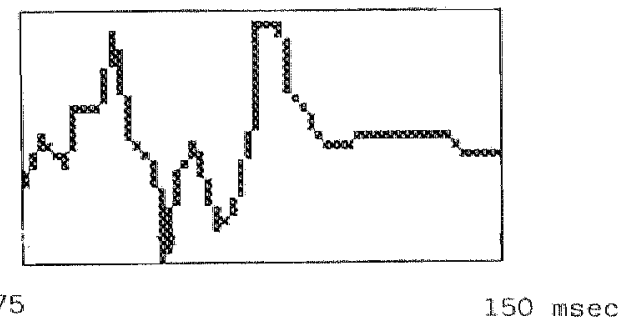

figure 5.10: Example of the digitization of one EMG signal. A: part of the original EMG trace on photographic record. B: EMG trace digitized between 75 and $150 \mathrm{msec}$.

\section{RESULTS}

All subjects received a constant stimulus intensity during the entire session, just slightly above the individual threshold for eliciting a reflex. This intensity ranged between 7.5 and $15 \mathrm{mAmp}$, with a mean intensity of $11.5 \mathrm{mAmp}(\mathrm{sd}=2.0)$. Stable reflexes of moderate intensity were produced in every subject.

All subjects $(n=11)$ had participated in the first counterirritation condition (CP test). The results for this stimulus are displayed in figure 5.11. The reflex magnitude during CP was on average $48.8 \%$ (sd: $29.0 \%$, range: $11.7 \%-91.1 \%$ ) of the magnitude in the control condition prior to stimulation. In the control condition afterwards, the reflex magnitude had returned to $95.7 \%$ (sd: $46.8 \%$, range: $54.3 \%-209.5 \%$ ) of the baseline value. The three conditions differed significantly from each other when tested with Friedman's anova $\left(x^{2}: 12.2, \mathrm{df}=2, \mathrm{p}=0.002\right)$. Post hoc analysis revealed that the CP 
condition was significantly different from both the first control condition (Wilcoxon, $z=-2.9, p=0.003)$ and the second control condition $(z=-2.3, p=0.02)$. The two control conditions did not differ significantly from each other $(z=-0.98, p=0.33)$.

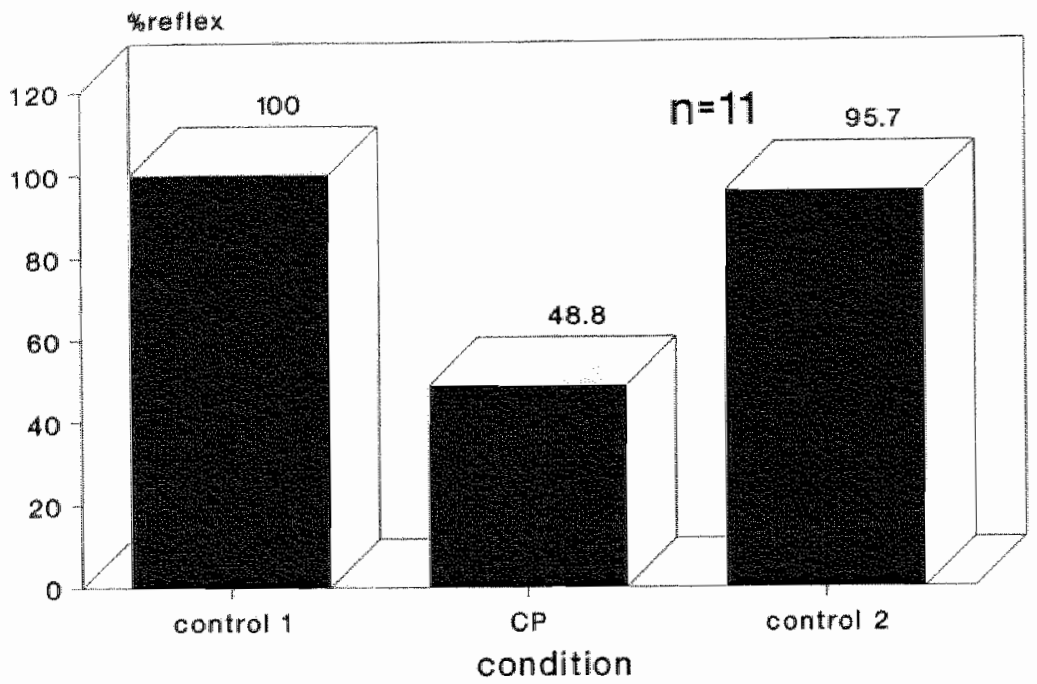

figure 5.11: Mean Alll magnitude in the cold pressor condition and the control condition afterwards, expressed as percentage of the control condition before counterirritation

Nine subjects participated in the tourniquet condition. However, in two subjects the tourniquet test did not result in pain and these subjects were left out of the analysis. The results are displayed in figure 5.12. Mean reflex mannitude for the tourniquet condition was $25.2 \%$ (sd: $17.8 \%$, range: $4.6 \%-52.1 \%$ ) of the magnitude during the control condition prior to it. In the control condition afterwards, the reflex magnitude had only partly recovered, namely to $71.6 \%$ (sd: $31.8 \%$, range: $25.9 \%-124.6 \%$ ) of the magnitude in the prior control condition. Again, the three conditions differed significantly from each other (Friedman"s anova, $\chi^{2}: 11.14, \mathrm{df}=2, \mathrm{p}=0.004$ ). Post hoc analysis between the conditions separately showed that the tourniquet condition differed significantly from the control condition before $(z=-2.4, p=0.02)$ and from the control condition afterwards $(z=-2.4, p=0.02)$, whereas the two control conditions showed a non-significant difference $(x=-1.7, p=0.09)$.

For the seven subjects who participated in all five conditions (excluding the two subjects who did not experience pain in the tourniquet condition), a comparison between all conditions combined was made. This allowed us to compare the relative effects of the two counteritritation stimuli. The refiex of the first control condition was again set to $100 \%$. The results are shown in figure 5.13. The five conditions differed significantly 
from each other $\left(x^{2}: 18.7, \mathrm{df}=4, \mathrm{p}<0.001\right)$. During the $\mathrm{CP}$ test, the reflex was reduced to $45.2 \%$ (sd: $30.7 \%$ ) for these 7 subjects. After the CP test, the reflex recovered completely (mean: $103.7 \%$, sd $56.7 \%$ ). The tourniquet test was shown to have an even greater effect, the reflex magnitude was reduced to $26.4 \%$ (sd: $20.1 \%$ ) of the initial control value. Also, there seems to have been a longer affereffect, because during the last control condition, the reflex had not yet restored completely (mean: $77.4 \%$, sd: $56.6 \%$ ). Thus, overall the tourniquet test had a somewhat greater effect than the CP test.

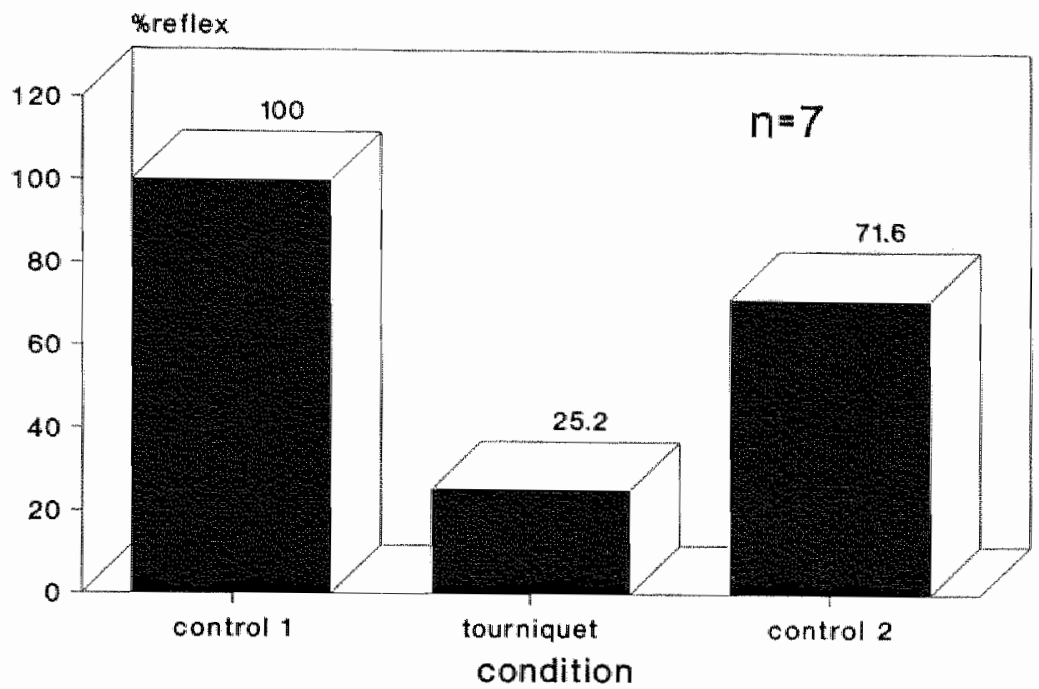

figure 5.12: Mean Rlll magnitude in the taurmiquet ischemic pain condition and the control condition afterwards, expressed as the percentage of the control condition before counterirritation

As can be seen from the large standard deviations, there was great inclividual variation in the efficacy of the counterirritation stimulus to depress the reflex. Nevertheless, individual analysis showed, that in most subjects inhibition of the reflex did in fact occur. Effective inhibition of the RIII was defined as a reduction of this reflex during the CI condition by at least $30 \%$, and a recovery of the reflex afterwards, also by at least $30 \%$. By this criterion, CP was effective in producing counterirritation in 9 out of 12 subjects. With the tourniquet test, in 6 out of 7 subjects a counterirritation effect could be established. Again, the tourniquet test appears to be somewhat more effective. As expected, there was no inhibitory effect for the two subjects who did not experience pain during the tourniquet test.

Individual curves for the 7 subjects who participated in all conditions are shown in figure 5. 14. 


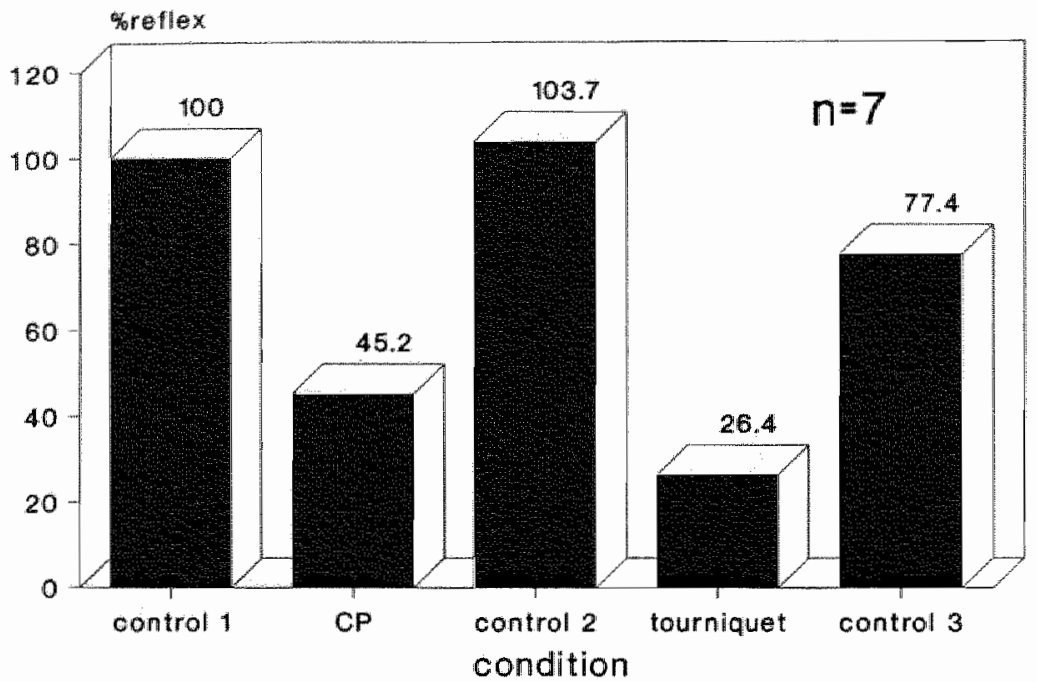

figure 5.13: Mean RIll magnitude during all five conditions, expressed as the percentage of the first control condition

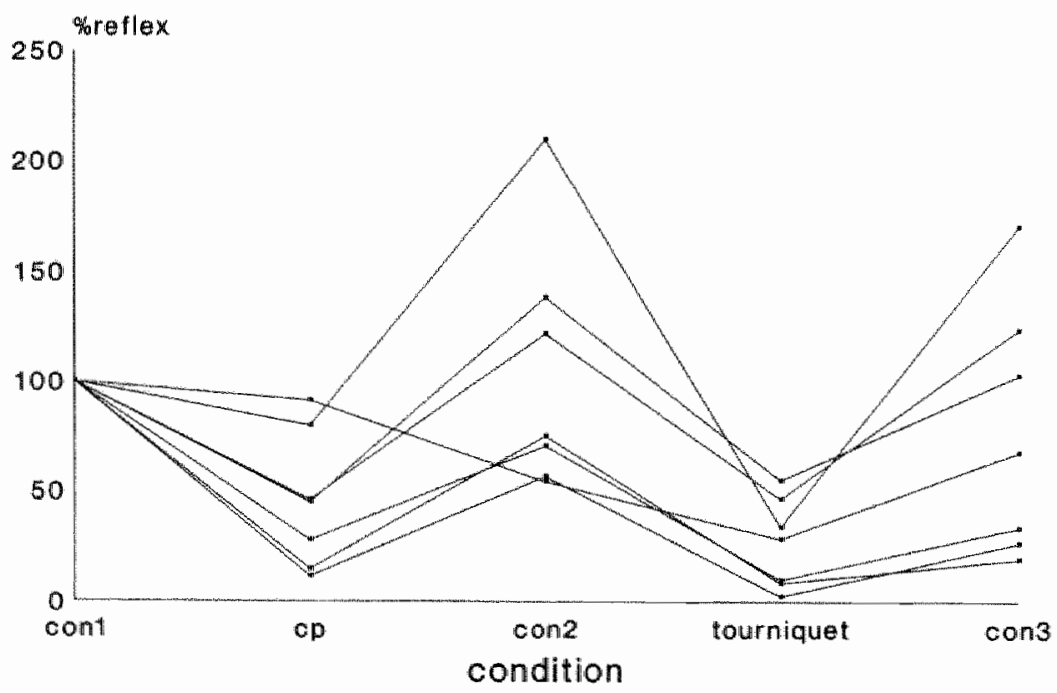

figure 5.14: Curves for indiwidual subjects participating in all five condlitions 


\section{DISCUSSION}

This study has confirmed that various pain stimuli are able to depress the RIII reflex. Depression of the related electrical pain sensation has not been tested. Both stimuli used in this study, cold pressure pain and ischemic pain, were shown to be effective as counterirritation stimulus. It appeared that the tourniquet test had a somewhat more powerful inhibitory effect on the RIII reflex than the CP test. This might have been due to the wider spatial distribution of the tourniquet pain stimulus in comparsion to the $\mathrm{CP}$ test (the whole arm versus the hand), or to the induction of more intense pain by the tourniquet test. Both factors are important in determining the magnitude of the counterirritation effect (Willer et al., 1989). Whether the tourniquet test did in fact produce more pain in our subjects is not clear. We did not make them score painfulness of the counterirritation stimulus afterwards.

The mean depressive effect on the RIII reflex of the two stimuli agreed well with previous findings with noxious heat (Willer et al., 1989; Roby-Brami et al., 1987). Between subjects, the degree of depression varied considerably. This is not surprising, because several variables were not quantitatively controlled for. The intensity of the electrical stimulus eliciting the reflex ranged from $7.5 \mathrm{mAmps}$ to $15.5 \mathrm{mAmps}$. Although this was always supra-threshold, the percentage above threshold was not fixed. Previous studies have kept the relative stimulation intensity constant, usually $20 \%$ above the threshold for eliciting the reflex (Willer et all., 1989; Roby-Brami et al., 1987). As a consequence of the variation in relative stimulation intensity in this study, the initial magnitude of the reflex varied between individuals too. Whereas some subjects had rather small reflexes, others had considerably larger ones. The ease with which a reflex can be inhibited might vary with initial reflex magnitude.

Another variable which was not controlled for, was the intensity of the counterirritation stimulus. Recording of the reflex was started when the subject reported pain, and stopped when pain tolerance was reached or after 20 recordings of the reflex. Subjects who reached pain tolerance before 20 recordings were made, might have experienced more pain than subjects who could easily endure 20 recordings of the reflex. The combination of initial variation in reflex magnitude and variation in the painfulness of the counterirritation stimulus makes differences in degree of depression belween subjects understandable.

Although the amount of inhibition differed, it was shown that the counterirritation effect could be demonstrated in most of the subjects tested. The tourniquet test in particular, was very effective, it induced a depression of the reflex in 6 out of the 7 subjects who experienced it as painful. In the two subjects in which the tourniquet test did not induce pain, the inhibitory effect on the RIII reflex was absent. This confirms previous studies, in which it has also been demonstrated that only clearly nociceptive stimuli induced counterirritation.

We conclude that the effect of counterirritation on the spinal RIII reflex is powerful and well reproducible. 


\subsection{CIIRONIC BACK PAIN, ACUTE POSTOPERATIVE PAIN AND THE ACTIVATION OF DIFFUSE NOXIOUS INHIBI- TORY CONTROLS (DNIC)'}

\section{SUMMARY}

The effect of the presence of either chronic or acute clinical pain on pain threshold and on the nociceptive flexion reflex (RII) threshold was studied. The experimental pain sensation and the flexion reflex were were evoked by trains of short electrical pulses. It was hypothesized that both kinds of clinical pain would be able to induce "diffuse noxious inhibitory controls" (DNIC), and thereby raise the two experimental thresholds. Patients with chronic low back pain, patients with postoperative pain from oral surgery, and pain-free subjects were tested in three conditions: during baseline, after i.w. administration of a placebo, and after i. $v$. administration of naloxone. In comparison with two pain-free control groups, the two pain groups had a significantly higher pain threshold in all conditions. However, the RII-threshold was not significantly elevated in chronic or acute pain patients compared to controls. Naloxone had no effect on the RIII- or pain threshold in any of the groups. It is concluded that the increased pain threshold which is frequently found in chronic pain patients, and which could be confirmed in the present study, does not result from a DNIC effect. The adlaptation level theory offers an alternative explanation. Also, the acute positoperative pain in this study did not seem to induce DNIC. Because other forms of acute pain have been found to be effective in activating DNIC, future research should establish which pains are and which pains are not effective.

\section{INTRODUCTION}

Several studies have demonstrated that chronic pain patients have an altered sensitivity to acute pain. However, there has been controversy about whether this is characterized by increased or rather decreased pain sensitivity. The parameter which has been assessed in the various studies seems to be a major factor determining the direction of the difference. With pain threshold experiments, the most consistent finding appears to be decreased sensitivity in chronic pain patients (Merskey \& Evans. 1975; Wolskee \& Gracely. 1980; Naliboff et al., 1981; Cohen et al., 1983;

'Peters, M.L. Schmidt, A.JIM., wan den Hout, M.A., Koopmans, R. \& Sluijter, M.E.. Chronic low back pain, acute postoperative pain and the activation of diffuse noxious inhibitory controls (DNIC), Pain (1992) in press. 
Yang et al., 1985). Consistent with this, in our own research with Chronic Low Back Pain (CLBP) patients, we found higher pain thresholds for both electrical pain stimuli and pressure pain stimuli (Peters et al., 1989; Peters \& Schmidt, 1992). At least two mechanisms have been suggested to explain these findings. The first mechanism is based upon the adaptation level theory, formulated by Helson (Helson, 1964). In this interpretation, the judgement of a pain stimulus is considered to be dependent on the context in which the stimulus occurs. In pain patients, the context is a painful state. This leads to the subject setting higher "internal anchor points", and subsequently judging additional pain stimuli as relatively mild (Rollman, 1979).

The second mechanism which has been proposed to explain the higher pain threshold in chronic pain patients, is a neurophysiological one "namely "diffuse noxious inhibitory controls" (DNIC), which has been extensively studied in the rat (Le Bars et al., 1979). DNIC involves the inhibition of nociceptive activity at the spinal level as a result of descending controlls from the brain stem, activated by noxious stimulation on any part of the body. Although originally formulated to explain heterotopic inhibition as a result of an acute noxious stimulus, it was suggested that it might also underly the higher pain threshold in chronic pain patients (Le Bars \& Willer, 1988). The purpose of the present study is to test this neurophysiolog. ical interpretation of raised pain thresholds in chronic pain patients.

Several studies have confirmed that DNIC can be induced in humans by applying two simultaneous experimental pain stimuli. More or less tonic pain stimuli decrease the perception of other (usually more phasic) pain stimuli (Anderson et al, 1976; Chen \& Treede, 1985; Chen et al., 1985; Talbot et al., 1987; Pantaleo et al., 1988; Price \& McHaffie, 1988; Riley \& Levine, 1988). The degree of inhibition of the phasic pain stimulus is directly dependent upon the strength of the tonic pain stimulus. Not only pain perception has been found to be reduced by an additional pain stimulus, but also the nociceptive flexion reflex (RIII-reflex), which has been found to correlate strongly with subjective pain perception (Willer, 1977). The reduction of the RIIIreflex suggests an effect on spinal nociceptive processes (Willer et al., 1984; RobyBrami et al.4 1987). Moreover, the opiate antagonist naloxone can abolish the reduction of the nociceptive reflex and the reduction of subjective pain perception induced by an additional pain stimulus (Willer et al., 1990). This parallels the findings obtained from DNIC studies in the rat.

Although for the most part acute experimental pain stimuli have been used to study DNIC in both humans and animals, a number of experiments have shown that clinical pain might also be effective in activating DNIC. In rats for instance, the threshold for heterotopic thermal nociceptive responses was increased after experimental induction of polyarthritis (Colpaert, 1979; Calvino \& Le Bars, 1986; Millan et al. "1987, Menetrey et al. 1988). In sciatica patients, a decrease in magnitude of the RIII-reflex was observed after the painful straight leg raising procedure (Willer et al, 1987). Finally, in subjects who experienced postoperative pain after menuscal surgery, the threshold of the RIII-reflex was substantially higher than that usually obserwed in normal subjects (Willer et al., 1985).

The present study further investigates whether clinical pain can induce DNIC. Patients with acute postoperative pain resulting from oral surgery and patients with 
chronic low back pain will be compared to pain-free controls with respect to their subjective pain threshold and RIII-threshold. In contrast to the adaptation level mechanism, which is a purely perceptual phenomenon, DNIC should lead to increases in both thresholds. A mixed between/ within subject design is employed, with every subject participating in three conditions: a baseline condition, a placebo condition and a naloxone condition. The evidence that the (eventual) higher thresholds in the two pain groups are the result of DNIC will be strengthened if it can be shown that naloxone reduces these thresholds only in the two patient groups (by blocking DNIC). Naloxone has no effect on experimental pain sensitivity by itself (El-Sobky et al., 1976; Grevert \& Goldstein, 1978; Stacher et al., 1988) and therefore will not influence the thresholds in the control group. The placebo condition is included to control for an eventual non-specific effect of injection upon pain sensitivity.

The three main hypotheses of this study can be formulated as follows:

1. During the baseline and placebo conditions both pain groups will have a higher pain threshold as well as a higher RIII-threshold as compared to the control group.

2. Relative to the baseline and placebo condition, in the naloxone condition pain threshold and RIII-threshold decrease in the two pain groups, whereas it remains constant in the control group.

3. There is a positive relationship between the degree of clinical pain and the height of the RIII- and pain threshold.

In addition, two subsidiary issues are adressed in the study. First the assumption that the RIII- and pain thresholds are significantly related to each other is checked. Second, the finding of several previous studies that naloxone has no effect on the intensity of the clinical pain (Lindblom \& Tegner, 1979; Langemark, 1989) is tested.

\section{METHODS}

\section{Subjects}

46 male subjects participated in the study. Beforehand, on the basis of a power analysis, it was determined that each of the three group should contain 12 subjects. However, a total of 22 oral surgery patients had to be tested to achieve a final sample size of 12 subjects in this group. Nine patients did not experience any pain or only very little pain after the surgical procedure. These nine patients. were excluded from the acute oral pain group, and assigned to an extra group (control group 2). The subjects in this group were identical to the acute pain group in all respects except for the pain they experienced afterwards. One other oral pain patient dropped out halfway during the experiment because the pain became intolerable.

The CLBP group consisted of patients suffering from daily backaches for at least one year. In all instances, the cause of the backache was unknown. Ten of the 
patients were recruited from the specialized pain treatment departments of two hospitals and from a revalidation center. Two patients were approached on the basis of their prior participation in experimental research. Only those patients were selected in whom no major psychological or psychiatric problems were known to exist. Most patients had previously taken part in an extensive psychosociall assessment procedure for diagnostic purposes. Seven patients were currently being tested for nerve blockade treatment. Most of these seven patients had had one or more previous blockadles. However, in all instances (part of the pain had remained. Three patients had participated in a behavioural or cognitive-behavioural treatment program at the revalidation center. Physical condition and mobility had increased and psychological and physical disability had decreased after treatment, but in all instances, pain had remained (although the patients indicated that they could control it better). Two patients had had no specialized treatment for their pain. The average duration of back pain was 13.4 years (range: $4-32$ years). In six patients the pain had increased over the years, whereas it had decreased in two of them. In two cases the pain had started after an accident, in two cases after a supposed disc prolapse, and in one case after the surgical removal of the coccyx.

All patients had pain predominantly in the lower back, with emphasis on the left side in four patients and on the right side in one patient. Frequently, pain radiated to one or both legs ( 9 times), twice also to the shoulders, and in one patient to the neck region. Only five patients did not use medication for their pain, five used NSAID's when necessary, two used benzodia-zepimes daily, and one patient used tricyclic antidepressants daily. No analgesics were used on the day of testing. All CLBP patients reported experiencing back pain throughout the experimental session (see also Results).

The oral surgery patients were recruited from the department of oral surgery. A standard procedure was selected, namely the surgical removal of a mandibular third molar. Testing began two to three hours after the local anaesthetic (ultracain $5 \%$ ) had been given. If a patient did not experience pain at the beginning of the experiment, or very little pain, he was assigned to the second control group. All subjects were asked to postpone the use of the prescribed analgesics untill after the experiment. One subject could not comply and he was excluded from the study. No other medication was used by the subjects in this group, except for one subject who used an antihistamine for his allergy.

The first control group consisted of 12 pain-free, healthy subjects. They were recruited by means of announcements in several university buildings or after direct approach by the experimenter. The subject group was made up of seven students, two university collaborators and three miscellaneous subjects. Only one subject in this group used medication, namely a drug against peptic ulcers.

Finally, the second control group was made up of the nine oral surgery patients who did not, or hardly did, experience any pain two to three hours after the operation (i.e. a score of $30 \mathrm{~cm}$ or less on a VAS scale during the baseline and placebo condition). The mean VAS pain score of these subjects during the baseline and placebo condition was only 6.6 , and they also indicated verbally to experience little discomfort. Even after the completion of the experiment, none of them deemed it necessary 
to take the analgesics they had to bring with them. No subject in this group used any other medication.

Exclusion criteria for all patients were general thealth problems (besides chronic pain), and cardiac complaints in particular. Screening took place by means of a health checklist which was completed by the subject before his admittance to the experiment, and by means of a short medical examination by a physician at the beginning of the experiment.

All subjects were male, to avoid possible circamensual variation in either pain perception or reflex threshold. Matching the three groups for age was not possible. The surgical removal of a wisdom tooth usually concerns young adults. Chronic back pain on the other hand, is not a common problem at that age. To keep the age difference as small as possible, the "eldest" oral surgery" patients awailable (and at least not under 20) were approached for participation. More details on age are presented in the result section.

Before the experiment, informed consent was obtained from every subject. After the experiment they all received a small financial compensation.

\section{Apparatus}

The RIII reflex was elicited and recorded by a Medelec MS6. The output side of the system consists of a stimulator, which generates constant current pulses. Current intensity can be adjusted from 0 to $50 \mathrm{mAmp}$ in steps of $0.5 \mathrm{mAmp}$. Beckman $\mathrm{Ag} / \mathrm{AgCL}$ electrodes, filled with isotonic Parker Spectra 360 electrode gel, were used for stimulation.

The EMG signal was recorded by means of disposable medicotest electrodes and fed into a small pre-amplifier near the site of recording. After additional amplification, the signal was bandpass filtered $(32-3200 \mathrm{HZ})$, and subsequently fed to an oscilloscope and optionally to a photographic recording unit. Oscilloscope and paper display occurred with a sensitivity of $20 \mathrm{mV}$ per $\mathrm{cm}$. All components of the system were mounted on a trolley which was placed out of sight of the subject.

Heart rate (HR) and blood pressure (BP) were reconded by a "Finapress" electronic recorder. This apparatus registers vascular activity with a small cuff around the middle finger. Systolic and diastolic BP, mean BP, and HR are visualized on a display for every beat. A paper recorder can print the $\mathrm{BP}$ trace, together with the average systolic BP, diastolic BP, mean BP, and HR for every 8 beats.

\section{Dependent variables (RII- and pain threshold)}

The RIIL-reflex was elicited in a manner similar to that used by other experimenters (Hugon, 1973; Willer, 1977). A train of 8 square 0.5 msec pulses with an internal frequency of $200 \mathrm{~Hz}$ was delivered to the skin above the nervus suralis just posterior to the lateral malleolus of the right foot (in CLBP patients with pain radiating into the right leg, stimulation and recording was performed on the left side). The cathode was 
placed proximally, and the anode distally. The repetition frequency of the pulse train was $0.2 \mathrm{HZ}$, i.e. there was an inter-trial interval of 5 seconds. The site under the electrode was mildly abraded with sandpaper, and cleaned with alcohol.

EMG recording took place by means of surface electrodes above the biceps femoris in the bend of the knee. The proper electrode site was identified by palpation, and the skin was prepared as described above. The scope displayed single sweep EMG activity of 225 msecs duration, triggered every 5 seconds by the pulse train. This same $225 \mathrm{msec}$ signal could also be recorded on photographic paper. The occurrence of the RIII-reflex was identified as a multiphasic deflection appearing on the scope within a time window of 75 to 150 msecs post-stimulus.

The RIII-threshold was determined by the presentation of a sequence of three ascending stimulus series. The minimal current in each series which could elicit a reflex was averaged for the three assessments and this average was taken as the RIIIthreshold. The EMG sweeps during the last of the three stimulus series were recorded photographically, and these recordings were scored afterwards for the occurrence of a reflex (for an example of the photographic recordings, see figure 5.15). Direct visual identification of the reflex from the scope proved to be very reliable. In almost all instances, the value obtained by scoring the reflex from the photographic recording afterwards, was identical to the one originally identified from the scope.

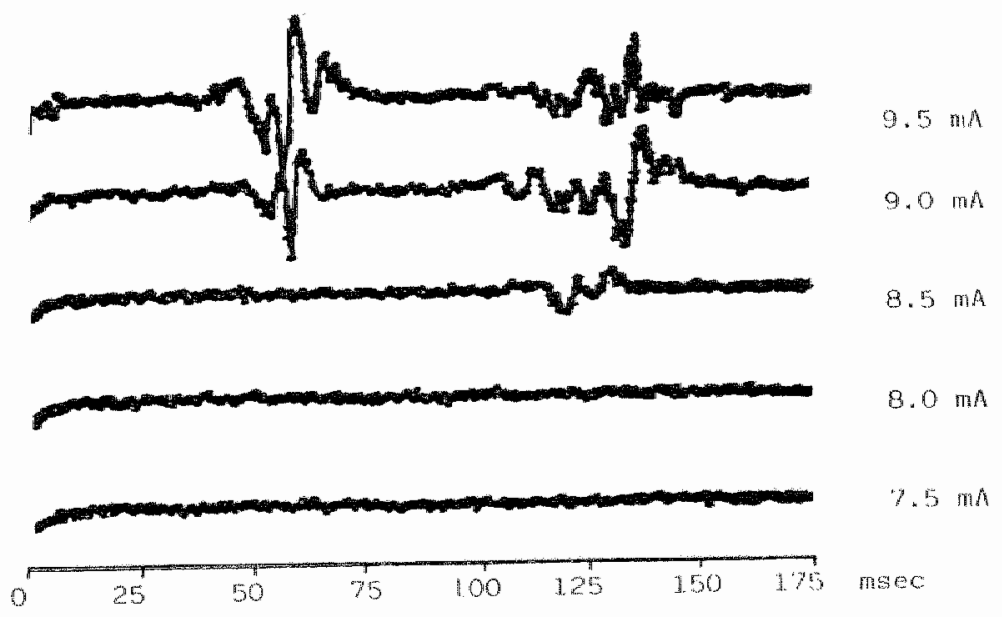

figure 5.15: Example of a photopraphic recording of part of a ascending stimulus series for one control subject. The EMG signal from the end of the electrical simulus until 175 msec afterwards is displayed. Stimulus intensity inceases from bottom to top of the figure. It can be seen that the first reflex activity begins to appear at a stimulus intensity of $8.5 \mathrm{~mA}$ for this subject.

During all stimulus presentations, subjects were instructed to concentrate on the sensation in the foot, and to report when they felt the stimulus to become painfull for the first time. It was stressed that they were to report the first feeling of pain, and that it 
was not a test of tolerance. The experimenter clarified that the sensation of pain would probably be like a sharp prick under the electrode. In the same way as RIII-threshold, pain threshold was defined as the minimal current at which pain was reported, averaged over three successive assessments.

HR and BP were measured to control for differences in arousal levell between the groups. An alternative explanation for RII- and pain threshold increases could be stress-induced analgesia (Tricklebank \& Curzon, 1984; Terman et al., 1984). During five different phases of the experiment (baseline, during familiarization when trains of electrical stimuli were given, after placebo injection, after naloxone injection, and when all assessments were completed) $\mathrm{HR}$ and $\mathrm{BP}$ registrations were made. Three 8-beat averages were recorded in every phase, and the mean of these three values was calculated for all variables.

\section{Procedure}

Before the experiment, all subjects completed a medical screening checklist at home. In addition, back pain patients also completed a back pain questionnaire.

At the beginning of the experimental session, a brief physical examination of all subjects was performed by a physician. During the actual testing procedure subjects were seated in a reclining chair, with the legs stretched and supported. Good muscular relaxation of the biceps femoris could be obtained in this position, as indicated by the resting $\mathrm{EMG}$ signal. In this reclining position, baseline levels of $H R$ and $B P$ were established.

The experimental session consisted of four phases. First there was a training phase, and subsequently three conditions during which the actual RII- and pain threshold determinations were made. The three conditions were always presented in fixed order, starting with the baseline condition, then the placebo condition, and finally the naloxone condition.

In the training phase the subject was familiarized with the electrical stimulus and the procedure for eliciting a reflex. Training took approximately 10 to 15 minutes, during whot several ascenuling senies or stimulus trains were given until the appearance of the reflex was definitely established. Also, the subject learned to identify his own pain threshold during this period. The training session was concluded with a 1.5 minute continuous stimulation period (one train every 5 seconds), at an intensity just suprathreshold for the eliciation of the RIII reflex. This stimulation period served two purposes. First, it eliminated much of much of the initial habituation which was seen while determining the RIII- and pain threshold. In addition, it allowed us to assess HR and $\mathrm{BP}$ during stimulation.

In the three experimental conditions, RIII- and pain threshold were measured three times with a two-minute interval between the measurements. As described above, all threshold assessments were based on three successive ascending series, and thus in every condition a total of nine of these series were given. For every series, stimulation intensity started at $0 \mathrm{mAmp}$ and gradually increased towards higher current levels. The steps at which the current increment took place varied, to avoid that subject's pain reports were biased by the number of stimuli they recelved. However, as the RIII- and 
pain threshold were approached, further increases were in equal steps of $0.5 \mathrm{mAmp}$. Stimulation increased until a clear RIII was seen on the scope and after the subject had judged the stimulus as painful. When both thresholds had been reached, the current intensity was again reduced to zero, and a new ascending series was started. After three of such series (i.e. one threshold determination) a two-minute interval took place.

In the first (baseline) condition, the RIII- and pain thresholds were established without any experimental manipulation. Next, in the placebo condition, $2 \mathrm{ml}$ of saline was slowly injected into the arm (i.v.). Shortly afterwards, HR and BP recordings were made. Starting five minutes after the injection, RIII- and pain threshold measurement began.

The naloxone condition was always the last condition, to avoid an eventual aftereffect of the drug into a subsequent condition. A $2 \mathrm{ml}$ solution of $0.8 \mathrm{mg}$ naloxone was slowly injected i.v. into the arm, and HR and BP were recorcled. After 5 minutes, the threshold assessments began. The effect of naloxone has been shown to occur rapidly in previous experiments, and its pharmacological steady state is about 5 to 15 minutes after injection (Willer et al., 1990).

The entire experimental procedure took approximately 1.5 hours. The experiment was approved of by the Medical Ethical Committee of our institution and was carried out along the usual ethical rules.

\section{RESULTS}

Age

There was a significant age difference between the groups. The mean age for the control group was 28.3 yrs (range: $20-41$ ), for the CLBP patient group 45.8 yrs (range: 28-69), for the acute pain patients 25.4 yrs (range 20-34) and for the second control group 23.8 yrs (range: 22-26). Only the CLBP group differed from the other groups. However, it was shown that there was no significant regression between age and average RIII-threshold $(B=-0.08, p=0.60)$ or between age and average pain threshold $(B=0.16, p=0.29)$. Therefore, age was not entered as a covariate in the subsequent analyses.

\section{RIII-threshold}

The results of the nine successive RIII-threshold assessments during the three experimental conditions are displayed in figure 5.16. It can be seen that during all conditions the RII threshold is roughly similar for the CLBP group and the two control groups, whereas the RIII-thresholds in the oral pain group appear to be higher. Statistical testing was performed by means of a Manova analysis with 2 within subject factors (condition and trial within condition) and one between subject factor (diagnostic group). The within subject factors were submitted to a polynomial trend analysis, to test 
for linear trends (habituation). The effects of interest from this analysis are indicated in table 5.17 .

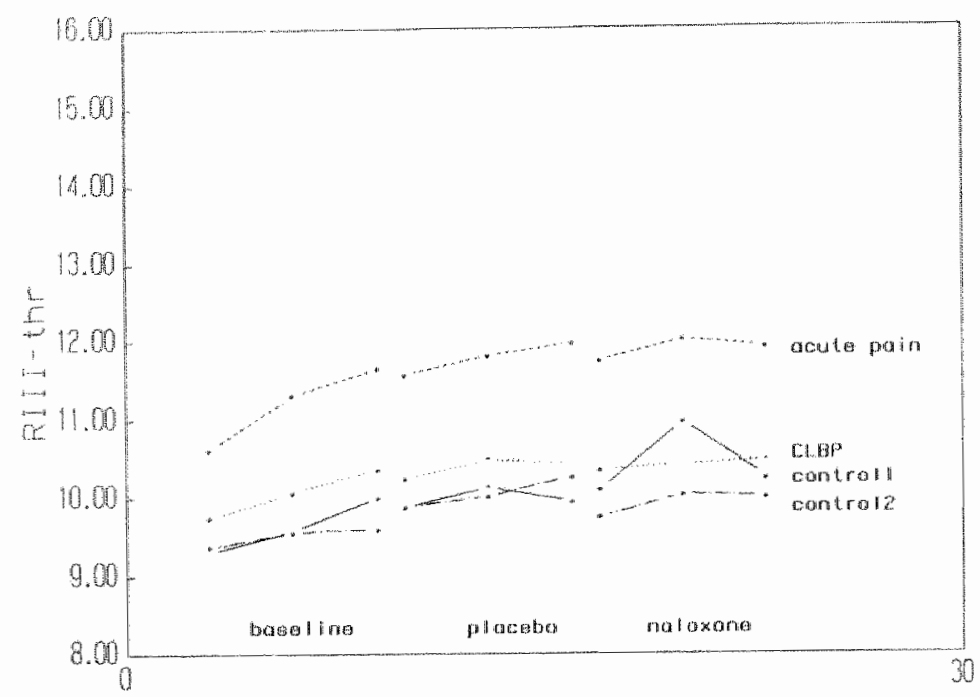

figure 5.16: The mean Rll1-reflex threshold for the four groups in the baseline, placebo and naloxone condition. Three threshold assessments were made in every condition.

\section{EFFECT}

main graup effect

main condition effect group $x$ condition effect linear condition effect lingar trial (within cond) effect linear group $x$ condition affect lingar group $x$ trial effect linesear triat $x$ condition effect

\section{Fidf)}

$F(3,41)=1.13$

$F(2,40)=6.96$

$F(6,80)=0.35$

$F(1.41)=6.08$

$F(1,41)=16.6$

$F(3,4)=0.16$

$F(3,41)=0.47$

$F(1,41)=6.78$ sign.

$$
\begin{aligned}
& p=0.348 \\
& p=0.003 \\
& p=0.912 \\
& p=0.018 \\
& p<0.001 \\
& p=0.929 \\
& p=0.705 \\
& p=0.013
\end{aligned}
$$

Table 5.77: Results of the MANOVA for the Rll-threshold data

The main group effect indicates the multivariate overall difference between the four diagnostic groups, that is over all trials and all conditions. In contrast to what appears from the figure, the four groups are not significantly different from each other with respect to the RIII-threshold. There is a significant main condition effect, which shows that the threshold of the RIII differs in the three conditions. However, the group by condition interaction effect is not significant, indicating that the changes 
between the conditions do not differ for the four groups. Such an interaction effect was in fact predicted. A higher RIII-threshold during the baseline and placebo conditions, but not in the naloxone condition, was hypothesized for the two pain groups. The results show that this hypothesis has to be rejected.

The two significant linear trends for the condition and trial factor demonstrate that habituation takes place both within and between the three conditions. The linear trends for the group $\mathrm{x}$ condition and group $\mathrm{x}$ trial effects are not significant, thus the degree of habituation does not differ between the groups. The last effect of interest is the linear trial $x$ condition effect. This effect reaches significance, and it can be seen from the figure that this is due to the levelling of within condition habituation in successive conditions.

\section{Pain threshold}

Figure 5.17 displays the effects of the 9 successive subjective pain threshold measurements which where obtained together with the reflex threshold. The same Manova approach was applied to the data, and the results of the tests of interest are indicated in table 5.18 .

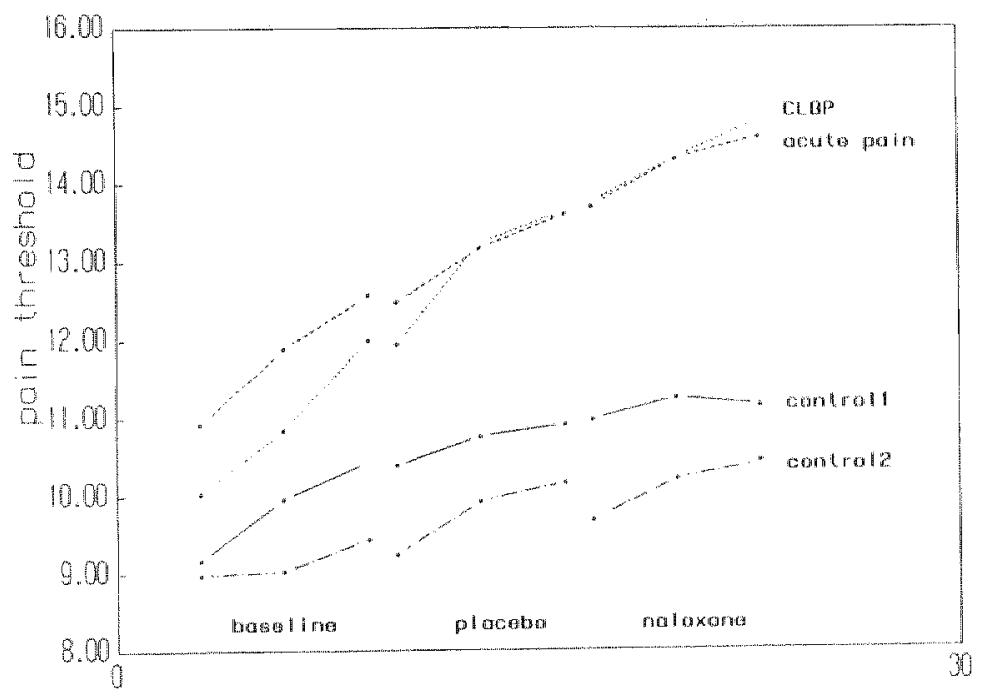

figure 5.17: Mean pain thresholds for the four groups in the baseline, placelbo and naloxone condition. Three threshold assessments were made in every condition.

Although the main group effect failed to reach the preset two-tailed significance level of 0.05 , a trend towards an overall multivariate group difference is apparent. In addition, from the figure it is clear that the differences between the groups were in 
the predicted direction, that is, with the two pain groups having higher pain thresholds than the two groups without pain. But again, the predicted group by condition interaction effect was not significant.

\begin{tabular}{lll}
\hline EFFECT & $F(d f)$ & sign. \\
\hline & & \\
main group effect & $F(3,41)=2.61$ & $p=0.064$ \\
main condition effect & $F(2,40)=19.8$ & $p=0.001$ \\
group $x$ condition effect & $F(6,80)=1.38$ & $p=0.234$ \\
linear condition effect & $F(1,41)=32.4$ & $p<0.001$ \\
linear trial (within cond) effect & $F(1,41)=71.1$ & $p<0.001$ \\
linear group x condition effect & $F(3,41)=2.44$ & $p=0.078$ \\
linear group x trial effect & $F(3,41)=3.20$ & $p=0.033$ \\
lineair trial $x$ condition effect & $F(1,41)=16.8$ & $p<0.001$
\end{tabular}

Table 5.18: Results of the MANOVA for the pain threshold data

The trend towards a main group difference was investigated further by means of several meaningful a priori contrasts. Because there was no group $\mathrm{x}$ condition interaction, one average pain threshold over all conditions was calculated for each subject. On this variable, the CLBP patient group was contrasted against control group 1, and against the combination of control groups 1 and 2 . Similarly, the acute pain group was contrasted against control group 1 and against control groups 1 and 2 combined. Finally, the two pain groups together were contrasted against the two control groups together. Thus, 5 contrast were specified. The results of the tests of these contrast are shown in the upper part of table 5.19 .

As can be seen from the table, only the contrasts involving the two control groups combined reached significance at a level of $\alpha=0.05$. However, when the Bonferonni correction for multiple comparisons is applied, multiplying the p-value by 5 (for 5 contrasts), only the last comparison, contrasting the two pain groups versus the two control groups, is significant.

The same group contrasts for pain threshold were specified within each condition separately. Similar results as for the overall test were obtained (see lower part of table 5.19). In all conditions, the comparison between the two pain groups and the two control groups was significant at $p=0.05$. Moreover, it can be seen that the differences increase during the session. During the last condition, all contrasts involving two control groups reached significance.

There were still some other effects of interest obtained from the Manova polynomial trend analysis. In accordance with the RIII-threshold data, the linear condition effect shows that the pain threshold habituated during the three successive conditions. Again, the linear trial effect was also significant, demonstrating within condition habituation. The linear group $x$ condition effect showed a trend towards significance, whereas the linear group $x$ trial did reach significance. Thus, habituation is different 
between the groups. Inspection of the figure shows that both pain groups appeared to habituate somewhat faster than the pain-free groups.

\begin{tabular}{|c|c|c|}
\hline CONTRASTS & $F(1,41)$ & signif. \\
\hline \multicolumn{3}{|l|}{ overall pain threshold } \\
\hline \multicolumn{3}{|l|}{ (mean value for the three conditions) } \\
\hline CLBP vs contr 1 & 2.57 & $p=0.116$ \\
\hline CLBP vs contr1 + 2 & 4.71 & $p=0.036$ \\
\hline acute pain ws contr 1 & 3.33 & $p=0.075$ \\
\hline acute pain ws contr $1+2$ & 5.76 & $p=0.020$ \\
\hline pain groups vs control groups & 7.69 & $p=0.008$ \\
\hline \multicolumn{3}{|l|}{ pain threshold during baseline } \\
\hline CLBP ws contr1 & 0.78 & $p=0.381$ \\
\hline CLBP vs contr1 +2 & 1.73 & $p=0.196$ \\
\hline acute pain vs contr 1 & 2.44 & $p=0.126$ \\
\hline acute pain vs contr $1+2$ & 4.31 & $p=0.044$ \\
\hline pain groups vs control groups & 4.20 & $p=0.047$ \\
\hline \multicolumn{3}{|l|}{ pain threshold during placebo } \\
\hline CLBP vs contr 1 & 2.63 & $p=0.113$ \\
\hline CLBP vs contr $1+2$ & 4.81 & $p=0.034$ \\
\hline acute pain vs contr 1 & 2.99 & $p=0.091$ \\
\hline acute pain vs contr $1+2$ & 5.35 & $p=0.026$ \\
\hline pain groups vs control groups & 7.42 & $p=0.009$ \\
\hline \multicolumn{3}{|l|}{ pain threshold during naloxone } \\
\hline CLBP' vs contr 1 & 3.99 & $p=0.053$ \\
\hline CLBP vs contr $1+2$ & 6.80 & $p=0.013$ \\
\hline acute pain vs contr 1 & 3.76 & $\mathrm{p}=0.060$ \\
\hline acute pain vs contr $1+2$ & 6.47 & $p=0.015$ \\
\hline pain groups ws control groups & 9.70 & $p=0.003$ \\
\hline
\end{tabular}

Table 5.19: contrasts between the groups for pain threshold

The significance of the trial $x$ condition interaction shows that also for pain threshold, within condition habituation decreases during the successive conditions.

\section{Clinical pain}

The mean clinical pain scores (either back pain or postoperative pain), obtained from the subjects four times during the experiment, are shown in table 5.20. Also, the average pain score over all trials is shown. The pain levell of the CLBP patients was constant during the entire experiment. There were no significant differences between the conditions in back pain level. The pain level of the oral patients on the other hand, was significantly different for the four conditions. Pain continuously increased (linear trend: $F(1,10)=34.7, p<0.001$ ). This can be explained by the fact 
that at the beginning of the session the local anaestethic was probably still partially active, and that during the course of experiment its effect gradually declined, increasing postoperative pain level.

\begin{tabular}{llll}
\hline & CLBP & oral pain \\
\hline pain before exper. & 36.3 & $($ sd: 24.6$)$ & 14.9 (sd: 9.5$)$ \\
pain after saline & 33.7 & $($ sd: 27.0$)$ & 30.5 (sd: 14.2$)$ \\
pain after naloxone & 35.6 (sd: 28.8$)$ & 42.0 (sd: 19.7$)$ \\
pain after exper. & 37.0 (sd: 25.7$)$ & 53.9 (sd: 21.2$)$ \\
average & 35.6 (sd:26.2) & 34.4 (sd: 13.9$)$ \\
\hline
\end{tabular}

Table 5.20: clinical pain levels for the three groups at 5 assessment periods

The relationship between clinical pain (either postoperative or chronic back pain) and the RIII-threshold and between clinical pain and pain threshold was tested by means of regression analysis. The mean VAS pain score for all four measurement periods was calculated for each subject in one of the two pain groups and entered as the predictor variable for the mean RIIl-threshold and mean pain threshold for all conditions. There was neither a significant regression between clinical pain and the RIII-threshold $(B=-0.28, p=0.18)$, nor between clinical pain and pain threshold $(\beta=0.12, p=0.51)$. Also within the two pain groups separately these variables were not related.

To investigate the possibility that our failure to find a difference in RII-threshold between the four groups was due to a pain level not sufficiently high to induce DNTC, another analysis was run on only those subjects with an average overall clinical pain score of 40 or more. For the two pain groups combined, this resulted in ten subjects. Compared to the first control group, the average RIII-threshold over all conditions. was not significantly higher for these subjects (mean RIJI-threshold for controls: 10.0, and for pain patients: $10.6, t=-0.48, d f=20, p=0.65$ ).

One of our subsidiary questions was whether naloxone has an effect on clinical pain. In the CLBP group, the pain score after naloxone did not differ from the other conditions, whereas in the oral pain group there was a monotonous linear trend, with no sudden extra increase in postoperative pain after naloxone.

\section{Cardiovascular variables}

Table 5.21 shows the mean $H R$, systolic and diastolic BP during 5 assessment periods for the four groups. There were no main group differences on any of the cardiovascular variables, nor were there any differences between the groups within the various conditions. 


\begin{tabular}{lccccc}
\hline & pre & pain & placebo & naloxone & post \\
\hline heart rate & & & & & \\
controll & 77.3 & 68.7 & 69.0 & 70.3 & 66.8 \\
CLBP & 76.7 & 72.3 & 75.3 & 73.2 & 70.0 \\
acute pain & 67.7 & 62.7 & 65.7 & 65.6 & 63.7 \\
control2 & 78.8 & 73.5 & 70.1 & 72.1 & 66.5 \\
systolic BP & & & & & \\
cantrol1 & 127.8 & 135.2 & 127.0 & 124.1 & 124.0 \\
CLBP & 121.1 & 127.9 & 128.4 & 128.5 & 119.8 \\
acute pain & 128.8 & 130.4 & 128.1 & 129.5 & 134.5 \\
control2 & 128.3 & 127.5 & 118.3 & 119.3 & 127.1 \\
diastolic BP & & & & & \\
control1 & & & & & \\
CLBP & 69.6 & 73.4 & 70.1 & 71.0 & 71.9 \\
acute pain & 74.1 & 74.0 & 71.1 & 73.0 & 70.7 \\
control2 & 72.2 & 6.8 & 71.1 & 74.4 & 74.3 \\
- & 70.3 & 66.4 & 67.1 & 65.9 & 69.8 \\
\hline
\end{tabular}

Table 5.21: Mean values for the cardiovascular variables during 5 assessment periods for the four groups

\section{Correlation between RIII-threshold and subjective pain threshold}

It has been reported previously that the RIII-reflex correlates highly with the subjective perception of pain. To test whether we could confirm this in the present study, two kinds of correlations between the RIII and pain threshold were calculated. First, a between subject correlation analysis was performed. This indicates whether subjects who had the highest RIII thresholds were also the ones with the highest pain thresholds. Pearson correlations were computed for all nine threshold assessments. The results of these analyses are shown in table 5.22. First, the correlations for all subjects are entered, and next for the four groups separately.

As can be seen from table 5.22, during all assessments, pain threshold and RIIIthreshold were significantly related in the subjects in control group 1 and in the acute pain group. For CLBP patients, a significant correlation was only obtained during the initial assessment but not during later trials. Also in control group 2, most correlations were lower than in control group 1 and the acute pain group, and mostly nonsignificant. However, the number of subjects in this group was lower.

The second correlation analysis was performed within subjects. For every individual the correlation between the 9 pain threshold scores and the 9 reflex-threshold scores was callculated. In order to enable the calculation of an average correlation for each group, the individual coefficients were first normalized by a $z$-transformation, and than averaged according to the formula given by McNemar (1969). This "averaged $z^{\prime \prime}$ can be transformed back to a normal correlation coefficient. The average within 
subject correlation confirmed the findings with the between subject correlations. In the first control group, the average correlation was $0.71(\mathrm{p}<0.05)^{2}$, in the acute pain group 0.67 ( $p<0.05$ ), and in the second control group $0.54(p<0.05)$. By contrats the average correlation in the CLBP group was only 0.22 (ns).

\begin{tabular}{|c|c|c|c|c|c|c|c|c|c|}
\hline assessment & 1 & 2 & 3 & 4 & 5 & 6 & 7 & 8 & 9 \\
\hline $\begin{array}{l}\text { all sulbjects } \\
(\mathrm{n}=45)\end{array}$ & $.76^{\circ *}$ & $.70^{\circ}$ & $.66^{\circ *}$ & $.66^{\prime \prime}$ & $.53^{* *}$ & $.53^{\circ \prime}$ & $.51^{* \prime}$ & $.47^{\circ}$ & $.47^{\circ}$ \\
\hline $\begin{array}{l}\text { control } \\
(n=12)\end{array}$ & $.80^{\circ}$ & $.80^{\circ *}$ & $.81^{* *}$ & $.81^{\circ}$ & $.75^{\circ}$ & $.76^{\circ}$ & $.81^{\circ}$ & $.81^{\circ}$ & $.85^{\prime \prime}$ \\
\hline $\begin{array}{l}\text { CLBP } \\
(n=12)\end{array}$ & $.61^{\circ *}$ & .25 & .04 & .15 & -.13 & -.13 & -.10 & -.12 & -.17 \\
\hline $\begin{array}{l}\text { acute pain } \\
(n=12)\end{array}$ & $.94^{* \prime}$ & $.87^{* 1}$ & $.89^{\circ}$ & $.85^{\circ *}$ & $.80^{\circ}$ & $.75^{\prime \prime}$ & $.75^{\circ}$ & $.69^{\circ}$ & $68^{\prime \prime}$ \\
\hline $\begin{array}{l}\text { Controll } 2 \\
(n=9)\end{array}$ & .33 & .40 & .47 & .47 & .41 & $.66^{\circ}$ & .50 & .58 & $69^{*}$ \\
\hline
\end{tabular}

Table 5.22: between subject correlations for RIII and pain threshold at the nine assessment periods. "indicates significance at the $a=0.05$ level and " indicates significance at $a=0.01$.

\section{DISCUSSION}

Our three main hypotheses could not be confirmed. The two pain groups were not significantly different from the control group(s) for both RMI and pain threshold during the baseline and placebo condition, and naloxone did not lower either threshold in the pain groups. Moreover, there was no relationship between degree of clinical pain and height of the RIII- and pain threshold. Thus, there is no evidence that the clinical pain in our subjects actiwated the DNIC mechanism. We will discuss this result in relation to chronic and acute pain separately.

The hypothesis that chronic pain would be able to induce DNIC was a tentative one. No prior experimental data were available to substantiate this assumption at the moment we set up the present study. The hypothesis was based mainly on the finding that chronic pain patients repeatedly have been found to have a higher pain threshold (Merskey \& Evans, 1975; Wolsky \& Gracely, 1980; Naliboff et al., 1981; Cohen et

\footnotetext{
The significance test of this average correlation is again based on the $z_{\text {avermo }}$ score.
} 
al., 1983; Yang et al., 1985; Peters \& Schmidt, 1992). Indeed, allso in this study, CLBP patients had higher subjective pain thresholds for the electrical stimulus as compared to the two pain-free groups. This was, however, not paralleled by increased RIII-thresholds. Therefore the higher subjective pain threshold does not seem to have resulted from DNIC, and an alternative explanation has to be advanced for this finding. A perceptual mechanism based upon the adaptation level theory seems to be the best hypothesis at present.

The finding that chronic pain does not trigger DNIC is consistent with another recent study which appeared during the time the present study was being carried out (De Bourreau et al., 1991). In this study, 53 chronic pain patients of various etiology were compared to 17 healthy controls on the variables RIII-threshold, subjective pain threshold and pain tolerance. Similarly to the present study, no difference was found between the groups for RIII-threshold, and there was no correlation between clinical pain and RIII- and pain threshold. However, there were neither any group differences on the subjective pain variables (pain threshold and tolerance). A significant difference was found for "unpleasantness threshold", i.e. the intensity at which $50 \%$ of the stimuli were judged as "weakly unpleasant" by a subject. This finding was interpreted by the authors as supportive of the adaptation level theory. They suggest that the adaptation mechanism had mainly affected the affective component of the pain experience in their experiment. In sum, the repeatedly found higher pain threshold of chronic pain patients is better explained by the adaptation level theory than by DNIC.

Similarly to the chronic pain group, in the acute oral pain group no significantly higher RIII-threshold was found, and there was no effect of naloxone upon RIII- or pain threshold. The most obvious conclusion is that acute postoperative pain cannot induce DNTC either. On the other hand, acute experimental pain has convincingly been demonstrated to be capable of reducing the RIII-reflex and pain perception (Le Bars et al., 1979c; Willer et al., 1984; Roby-Brami et al., 1987; Willer et al., 1989). We recently were able to replicate this finding ourselves, using two different experimental pain stimuli (Peters et al., 1991). Moreover, there have been indications that acute clinical pain can also decrease RIII-reflex amplitude and raise the RIII-threshold (see introduction). The fact that in the present study no evidence was found for the activation of DNIC in an acute pain condition, contradicts these previous findings. We can speculate on several explanations for this.

First, the nature of the stimulus in the present study was qualtitatively distinct from that used in most other DNIC studies. In our study, subjects experienced ongoing pain as opposed to phasic pain which is induced by experimental pain stimuli. Postoperative pain is also different from the sharp and short-lasting pain-stab which can be elicited in sciatica patients by straight leg raising, the latter being the procedure in the Willer et al. 1987 study. On the other hand, the pain experience after surgical removal of a mandibular third molar is probably comparable to that after meniscal surgery, which was used in the Willer et al. 1985 study. In this study, the RIII-threshold was found to be markedly elevated in patients with postoperative pain compared to the values which were found in normal controls in previous experiments. It should however be noted that the study only comprised four subjects, and that no separate control group was included in the experiment itself. The study was actually 
not degigned for the purpose of assessing RIII-threshold in an acute pain condition. To our knowledge, the present sudy is the firsit to systematically test the RIIIthreshold in patients with acute ongoing pain versus pain-free subjects. Acute pain of this specific nature simply may be unable to activate DNIC.

An alternative explanation for why in our study acute pain may not hawe triggered DNIC could be that the patients did not experience pain of sufficient intensity. As reported in the results section, oral surgery patients had an average pain score corresponding to a moderately painful level. However, separate analyses on only those subjects experiencing most pain, did not alter the result. In addition, it was shown that there was no significant regression between intensity of clinical pain and height of the RIII-threshold. "Therefore, this interpretation seems to be less likely.

Nevertheless, it cannot be excluded altogether that acute postoperative pain has a DNIC effect. Most DNIC studies have used the reduction of supra-threshold RIIIreflex amplitude as the dependent wariable. The RIII-threshold assessment procedure which was used in the present study may not be as sensitive to modulation by DNIC as measurement of RIII amplitude. If the effect on RIII-threshold is only small, our study would not have enough power to detect such an effect. Before the experiment, a power analysis was performed to determine the necessary sample size to demonstrate a minimal difference between the groups in RIII-threshold of $3 \mathrm{mAmp}$. On the basis of previous studies the sd was estimated to be 2 . With 12 subjects per group, a power of more than $90 \%$ could be obtained. However, with smaller effect sizes, the power rapidly decreases. As can be seen from the figure, a slightly elevated RII-threshold was obtained for the oral pain group, but the difference with the control groups only amounted to $1.7 \mathrm{mAmp}$. Moreover, the sd was higher $(2.8 \mathrm{mAmp})$ than estimated beforehand. The power of detecting such a dramatically lower effect size with 12 subjects per group would be less than .40! Thus there might actually have been a small elevation of RIII-threshold due to postoperative pain, but our test may not have been sensitive enough to demonstrate this effect.

In short, it is at present unclear whether postoperative pain can trigger DNIC. Postoperative pain may be qualitatively distinct from other kinds of acute pain known to induce DNIC, and unable to activate descending inhibitions. On the other hand, the lack of an effect of postoperative pain on the RIII-reflex can also have been due to the particular procedure which was used. Future studies can perhaps elucidate whether the activation of DN.C by acute postoperative pain can be demonstrated by means of RIII-amplitude measurements.

An interesting observation resulted from testing the subsidiary hypothesis that there is good correspondence between the RIII- and pain threshold. It was demonstrated that the two thresholds were significantly related in the acute pain and control groups, but less so in the CLBP patient group. The subjective and physiological responses to the electrical pain stimulus can be said to be "discordant" in chronic pain patients. A parallel can be drawn with the clinical situation. Usually, three components of pain are distinguished: a cognitive-subjective, a physiological and a behavioural compo nent. It has been observed that chronic pain patients often display a considerable degree of discordance between the various components of pain (Vlaeyen, 1991). Several explanations for this clinical finding have been advanced and these may be 
applied to the present observation of discordance between experimental pain variables as well. Gannon and Haynes have argued that discordance between the cognitive and the physiological system might result from an inability to perceive physical signals accurately. As a result, appropriate coping behaviour in response to these signals cannot be initiated, and physiological disorders may ensue. Hence, in this interpretation discordance is seen as a risk factor for several psychophysiological diseases, among which is chronic pain (Gannon \& Haynes, 1986). The inability to perceive physical sensations accurately can be generalized to the laboratory situation and thereby explain the discordance between the RIII-reflex and the subjective pain response observed in this experiment.

Alternatively, discordance between the various components of pain can develop because learning processes will affect these components differently. Pain behaviour is said to be under powerful operant control (c.f. Fordyce, 1983), whereas this is less apparent for the physiological component of pain. Verbal pain responses will also be influenced by prior operant learning processes, and probably more so in chronic pain patients than in healthy controls because of the more frequent experience pain patients have with environmental contingencies in relation to expressions of pain. When operant control on subjective pain report is strong, the relationship with the RIIIthreshold, which is not influenced by the same learning processes, will probably be weaker.

To summarize the main findings of this study, CLBP patients do have a significantly higher pain threshold than pain-free controls, not paralleled by a higher RIIIthreshold. Therefore, DNIC does not seem to be activated by chronic pain. The findings with acute postoperative pain are less clear-cut. In this study, no evidence for the activation of DNIC by pain from oral surgery was found. Future studies will have to establish under which circumstances acute pain is able to activate DNIC. Finally, it was shown that whereas in most subjects there was a strong correspondence between the RIII-threshold and the pain threshold, this correspondence was absent in CLBP patients. 



\section{DISCUSSION}

In the six previous chapters, various issues pertaining to chronic pain and pain perception have been addressed, both theoretically and empirically. Three major areas of interest were specified, which have been discussed in three successive theoretical chapters (chapters 2 to 4 ). These chapters concerned psychophysiological models of chronic pain, pain sensitivity in chronic pain patients, and habituation to pain stimuli by chronic pain patients respectively. Several of the remaining research questions were subsequently tested in nine empirical studies. The results of these studies, and how they relate to the questions specified at the end of each theoretical chapter, will now be discussed. Again, the three major topics will be treated separately.

\subsection{Psychophysiological models of chronic benign pain}

Of the various psychophysiological models of chronic pain which were discussed in chapter 2 , three have been addressed in particular in our empirical studies, namely the diathesis-stress model, the biomechanical model and the cognitive-physiological discordance model. Below, it will be discussed what our studies, in combination with some other recent studies, can add to the understanding of psychophysiological factors in CLBP. The discussion will mainly focus on the significance of muscular variables in CLBP, as this is probably the most fruitful line of research in psychophysiology. An attempt woll be made to present an integrated view of the various separate research findings, and to discuss the implications of this view for further clinical studies. In addition, a separate section will be devoted to the concept of cognitivephysiological discordance.

\section{Diathesis-stress model}

The study reported in section 5.2 was set up to test a major aspect of the diathesisstress model of chronic back pain, namely the prediction that CLBP patients show an increased muscle response in a relevant stress situation. The diathesis-stress model was considered very promising on the basis of previous research conducted by Flor et al (1985). In our study, we used a somewhat different design from this original work. Instead of discussing pain or stress situations as the experimental stressor, we employed an experimental pain stimulus (pressure pain), which we speculated to be a relevant stressor for pain patients. The main result of this study was a significant elevation of back muscle tension on the left side in about half of the CLBP patients during the pain test. However, this increased back muscle tension did not seem to be related to the stressor itself, but rather to a change in posture. Therefore, we have interpreted these results as consistent with the biomechanical model of pain, which 
assumes a different patterning of (back) muscle responses. Bielow, the biomechanicall model will be discussed in more detail.

Direct evidence for the diathesis-stress model was not found in our study. There was no increase in back muscle tension as a result of the pain stimulus in CLBP patients. However, our results can neither be taken as a falsification of the model. Two explanations can be given for the absence of an increased back muscle response in this study, without undermining the diathesis-stress model. First, the stressor we have used might not have been sufficiently relevant. One of the premises of the diathesis-stress model is that only personally relevant stressors induce the specific muscular hyperreactivity. Flor et al. (1985) demonstrated that only during the condition in which a subject had to recount either a recent pain or a recent stress episode, back muscle tension differentiated CLBP patients from controls, whereas during mental arithmetic no differences were found between the groups. We did not include a manipulation check in our study to establish the subjective stressfulness of the stimulus. However, several. lines of evidence suggest that chronic pain patients do experience pain stimuli as stressful, and even more so than healthy controls. In our study, CLBP patients did show a greater general arousal response (as shown by SCR) to the pressure pain stimulus. The same was found in one of our other studies (Arntz. et al. 1991) which was previously discussed in chapter 2. In this study, CLBP patients displayed stronger skin conductance and frontalis EMG reactions to electrical stimuli. Collins at al. (1982), using the CP' test with CLBP patients, likewise reported increased frontal and SCR reactivity to the pain stimulus in the patient group. Thus, CLBP patients do seem to become more aroused by a pain stimulus. In addition, in the Amtz et al. study the predicted increased paraspinal reactivity in response to the pain stimulus was found. Thus, the irrelevance of the stressor does not seem to be able to explain the absence of the predicted increased responsiveness to the pain stimulus.

The second explanation is based on a confounding factor, namely postural change, overruling possible stress-related changes. The major difference between the Arnitz et al. study in which an increased paraspinal response to a pain stimulus was found and the Peters et al. study in which this was not found, was the procedure of pain stimulation. The former study delivered electrical shocks to the subject's finger while (s)he remained immobile, seated comfortably in a reclining chair. No major postural change took place during the delivery of the pain stimuli. By contrast, in the latter study, subjects were seated at a table, and had to extend one of their arms to place the finger in the pain device placed on the table. Thus, postural changes which might have attenuated the muscle response to the stressor took place. A change in posture could have larger and longer-lasting effects on muscle tension than the probably short and phasic increases seen after a pain stimulus.

Since the first study of Flor et al, evidence for the diathesis-stress model has grown. The original study has recently been replicated several times by the same group. A very comprehensive study including 120 CLBP patients and 36 healthy controls confirmed that elevated paraspinal activity occurred during stressful imagery specifically in the CLBP patient group (Flor \& Birbaumer, 1991). In two studies it was shown that the diathesis-stress model is generalizable to another diagnostic 
category, namely to temporomandibular pain. One of these shidies concerned both CLBP and temporomandibular pain patients (Flor et al., 1991). Increased paraspinal reactivity (especially on the left side) during stressful imagery was found in CLBP patients, whereas increased masseter muscle reactivity was found in temporomandibular pain patients. Another study was directed at temporomandibular pain exclusively (Flor et al., 1991b). Again, in the stressful imagery condition, a greater masseter EMG reactivity was found in the patient group. However, also in at neutral imagery condition, there were significant differences in EMG reactivity between the groups, although less pronounced than during stressfull imagery.

In sum, it can be concluded that the evidence for the diathesis-stress model is gaining strength. One of our own studies (Arntz et al.) was consistent with this model, but the other (Peters et al., included in this dissertation) could not confirm the predictions of the diathesis-stress model. However, as discussed above, this might have been due to the strong postural effect which was found.

\section{Biomechanical model}

The results of the study reported in section 5.2 were interpreted as being consistent with the biomechanical model of CLBP, which predicts a faulty patterning of spinal muscular activity resulting from postural abnormalities developed during an acute pain episode. One manifestation of such abnormal patterning could be tensing the back muscles when it is not appropriate, that is, not necessary for performing a certain movement. In our study, some $50 \%$ of the subjects in the patient group seemed to have displayed such inappropriate paraspinal muscle contractions. However, our study was not originally intended to test the biomechanical model. As was outlined in chapter 2 , another type of study is needed to adequately test the model, with all of the hypothesized processes. Such studies should answer the question whether the abnormal muscle patterning results from a postural abnormality and whether the ultimate source of pain in CLBP patients is mechanical rather than directly related to muscle tension.

\section{Towards an integrated model}

The diathesis-stress model as well as the biomechanical model can explain a number of the findings in CLBP patients, but neither of these models can explain all findings. The biomechanical model nicely deals with findings pertaining to physical strain (posture related differences in muscle tension, altered patterning of dynamic muscle function), whereas the diathesis-stress model is especially suited to explain the findings related to mental strain (exaggerated muscle reponses in stress situations). In this section an attempt is made to integrate both models into one new model. This new model is presented in figure 7.1. Some new elements are included, but the two original models can be easily recognized. The upper part of the higure reflects the biomechanical model, which boils down to the assumption that incorrect posture and 
abnormal muscular function during movement cause mechanical irritation and thereby pain. A positive feedback loop is present in the system, which leads to exacerbation and maintenance of pain. In the lower part of the figure, the diathesis-stress model can be recognized. The causal chain of events here is that mental strain leads to repeated and prolonged muscular activation which ultimately may produce ischaemia and metabolic changes (accumulation of break-down products). Again, the result will be pain. In addition, the diathesis-stress model assumes a predisposition (diathesis) to react to stress with muscle contractions. This aspect has not been indicated as a separate element in the model for it is incorporated in the proposed physiological basis of the system. This will be explained later.

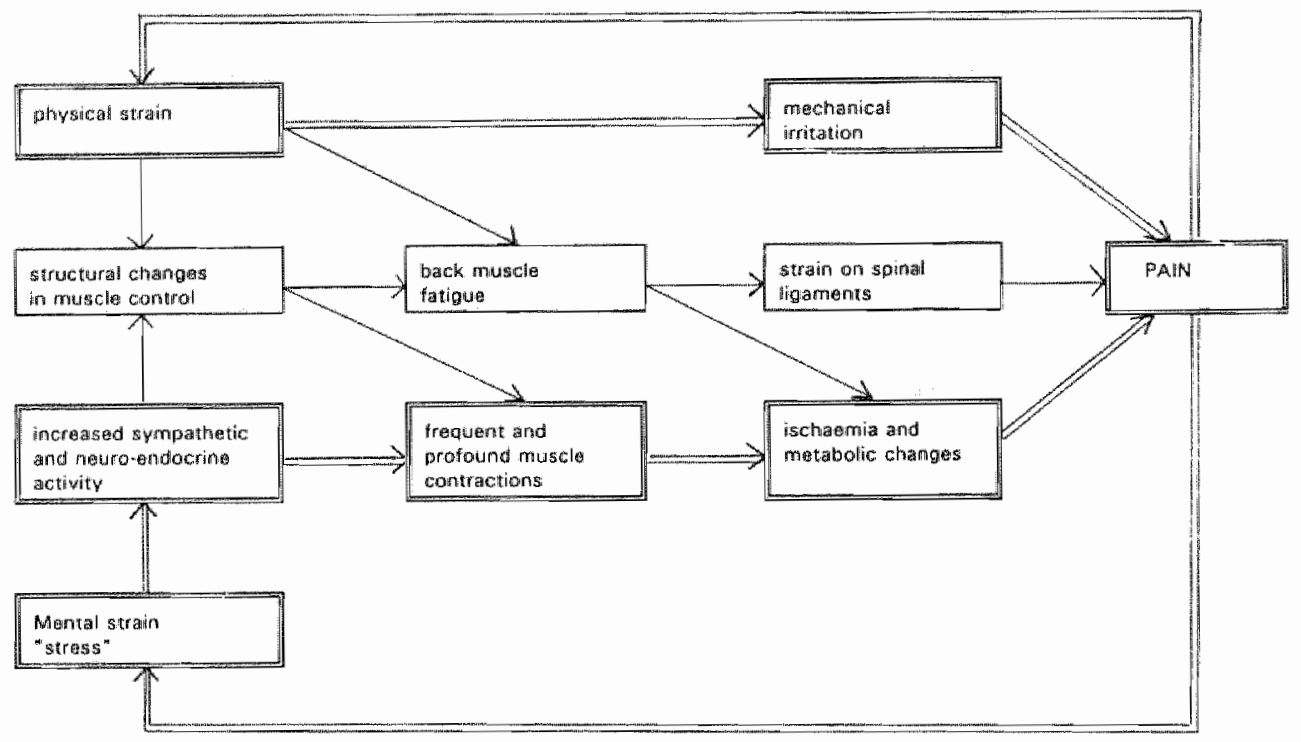

figure 6.1: An integrated model of chronic low back pain

As can be seen in figure 6.1 , besides the two potentially pain generating processes which are identified by the diathesis-stress model and the biomechanical model, the present model specifies a third process which could be responsible for pain in CLBP, namely spinal ligamentous strain. It is commonly assumed that such strain can occur when the back muscles do not provide adequate support, for instance because they are fatigued (Wyke, 1987; Cailliet, 1981). Thus, back muscle fatigue and spinal ligamentous strain form a third major route which may lead to back pain.

The core assumption of the present model is that for some reason muscle control has structurally changed in CLBP patients. This change in muscular control is supposed to constitute the diathesis in this model. It is proposed that the relative 
predominance of muscle responses after activation is due to biochemical changes within the muscle itself and alterations in the mechanisms of neuro-muscular regulation. These changes could lead to a state of the muscle in which it contracts readily and more forcefully. In a previous paper we have called this a state of "action-readiness" of the muscle (Arntz et al., 1991). Physical strain factors are supposed to be important for the structural changes in muscle control to occur. These factors can form one of the links between the biomechanical and the diathesis-stress model. In the biomechanical model postural abnormalities and aberrant pattems of movement are assumed to cause mechanical irritation and pain. However, these same physical factors could also be responsible for creating a hyper-excitable state of the muscle. Another proposed factor leading to changes in muscle control is mental strain. In the diathesis-stress model, mental strain is considered to be the activating stimulus which is expressed especially prominant in the muscular system because of a diathesis. In the present model, mental strain is considered one of the factors responsible for creating a diathesis by inducing more or less structural changes in muscle control. Exactly how physical and mental strain affect muscle control will be adressed only briefly, for this is not the place to go into muscle physiology in great length, or to sum up all mechanisms which could potentially influence responsiveness of the muscle. Just a few observations will be presented here.

First, overuse of a muscle has been suggested to lead to changes in the size and type of muscle fibers (Janda, 1978), as well as to biochemical changes. The latter does not only occur after prolonged overuse, but has also been found after a few days of unaccustomed exercise (Bengtsson \& Henriksson, 1989). Mental strain can affect muscle structure and biochemistry through the sympathetic nervous system (SNS). The SNS regulates micro-circulation of the muscle, and it has been speculated that sustained decreases in circulation will lead to edema and the accumulation of metabolites, and finally to structural changes in the muscle (Kirkaldy-Willis, 1988). There is also a direct sympathetic effect on regulation of muscle contractions. Adrenalin can increase the contractile force of the extrafusal muscle fibers, and both adrenalin and noradrenalin promote neuro-muscular transfer (Geers, 1988).

Alterations in muscle structure and biochemistry can have direct effects on the excitability of a muscle (e.g. lowering the threshold of activation, causing a more forcefull and prolonged response after activation). In the model therefore, a link is made between physical strain and mental strain, via structural changes in muscle control, with frequent and profound activation of the muscle. In addition, altered muscle control can also directly or indirectly be responsible for muscle fatigue. Changes in muscle biochemistry and in muscle fiber types (e.g. towards the so-called "fast-twitch fatiguable fibers") can make the muscle more prone to fatigue. The frequent and more profound activation which occurs in these muscles will further add to this fatigue. It was discussed above how muscle fatigue and frequent and prolonged muscle activation can lead to pain through two separate mechanisms.

To summarize, the basic assumptions of the model are that both mental and physical strain will contribute to back pain. At a physiological level, alterations of a biochemical nature and of neuro-muscular control will take place. Through altered activation patterns of the muscle (more frequent, more profound) and fatigue, but also 
as a direct result of physical strain, pain is generated. Three final pathways can be held responsible: (1) mechanical irritation of nerve root, capsular and joint structures, (2) ligamentous strain and (3) ischaemia/metabolic changes.

Some of the elements in the model have already been demonstrated to the associated with back pain, others are still largely speculative. On the left, the initiating factors and the physiological result thereof are indicated. Previous research has assessed whether either physical strain or mental strain is a feature of low back pain. To begin with the first, it is well known that many patients with back pain have an abnormal posture or move in an abnormal manner (e.g. Rosomoff et al., 1989), possibly in an attempt to alleviate pain. In addition, people with structural abnormalities (e.g. differences in leg lengthy or with back straining jobs are at a greater risk for developing back pain. The next element in the model, structural changes in muscle control, is still speculative at the moment. Regarding mental strain, indications have been found that many low back pain patients suffer from increased tension, anxiety or depression. Although this may be secundary to pain, it can lead to exacerbation and maintenance of pain. That stress can lead to sympathetic and neuro-endocrine activation does not need further comment.

On the next level, the demonstrable muscular consequences of these initiating processes are indicated. Muscle fatigue can be measured by means of power spectrum analysis of the EMG signal. It is expressed as a shift of the activity towards the lower frequency domain. At least one study has already found evidence for lumbar muscle fatigue in CLBP patients (Roy et al., 1988). In some of these patient's muscles the median frequency was already lower during rest conditions, while other muscles were fatigued more rapidly after isometric contraction. This confirmed earlier studies with the same results (see refs. in Roy et al.). Pertaining to the more frequent or profound phasic muscle activity, the abovementioned studies of Flor et al. have demonstrated convincingly that stress stimuli elicit more pronounced phasic muscle activity in CLBP patients than in normal controls. One of our own studies (Arntz et al., 1991) has confirmed this by means of a pain stimulus. But also a simple postural change and the activation of unrelated muscles was sufficient to provoke unexpected lumbar muscle responses in CLBP patients."

Finally, on the right side of the model, the potential sources of back pain are indicated. The processes specified here are most likely painful, structures with nociceptive inmervation are involved. However, these processes are not directly demonstrable and therefore their role in generating pain in CLBP patients remains speculative.

"A parallel can be drawn with studies with nervous and anxious people, in whom, in the performance of a simple motor task which normally requires the activation af only few motor units, a simultaneous discharge of motor units throughout the body was demonstrated (Calliet. 1981). Goldstein (1970) presents an overview of studies in which increased muscle responses to various stimuli (pain, noise, motor tasks] were found in tense and anxious people. Muscle control in these subjects might have changed in a similar way as in CLBP patients, namely towards hyper-excitability, because of sustained sympathetic and neuroendocrine activity. 
Except for the processes explicitely included in the model, still others may be involved. For instance, various psychological processes can modulate the experience of pain (see introduction). Moreover, the psychological make-up of an individual also determines how effectively (s)he will cope with stressors in general. Inadequate coping will cause a greater impact of stress on the system. Lastly, genetic factors can be thought to affect all levels of the process. Inherent structural deformations increase the risk of getting back pain. The genetically determined functioning of the muscle determines basic excitability at which the effects of strain are superimposed. And constitutional build will determine the susceptibility of certain structures for developing pain.

Does the model explain all back pains for which no demonstrable physical cause has been identified? Flor \& Birbaumer (1991) found that when assessing a group of 120 CLBP patients on several physical and psychological variables, and subsequently clustering them on the basis of the results into four different groups, only one of the groups was characterized by increased muscle tension level and increased phasic muscular responsiveness. This group contained a mere $8 \%$ of the total sample! This endorses the view that myogenic factors only play a predominant role in a minority of back pain patients. In other patients, other somatic and psychological factors might be of more importance. But although Flor \& Birbaumer report a very low percentage of patients in which muscular variables are the predominam factor, this might underestimate the actual situation. Firstly, the assessment might not have included all of the relewant parameters (e.g. no measurement of muscular fatigue or muscle activation during dynamic movement). Secondly, the patients in the other groups may have had a contribution from muscular factors, but other additional "abnormalities" were possibly more prominent. And finally, patient selection appears to be cruciall. In the Flor \& Birbaumer study, patients were recruited from a pain clinic. Possibly there is a referral bias to such a center of patients with operant control or other psychological factors maintaining the pain. In our study in section 5.2, a larger proportion of patients seemed to be characterized by a muscular dysfunction. An abnomally large increase in paraspinal muscle tension during postural change was displayed by half of the CLBP patients participating.

The model as it is presented here leads to several predictions. Theoretically, all direct and indirect relations between the elements can stand for a prediction, but some of these will be obvious or trivial, whereas for others it will not be possible to test them directly. However, a number of testable predictions can be deduced.

(1) Experimental induction of physical strain on lumbar muscles in normal subjects will lead to (temporary) biochemical changes which make these muscles more excitable. Thus, when the muscle is stimulated (for instance by a pain stimulus) a more pronounced phasic response in the particular muscle is expected after strain than without strain.

(2) Phasic muscle responses to a (pain) stimulus in normal controls will be more prominent in an experimentally induced stress condition than during relaxation or rest.

Fatigue will be found specifically in the lumbar musculature of CLBP patients. 
In other muscles no increased fatigue is present.

The more frequent and prolonged phasic activation of the muscle after stimulation is also specific for lumbar muscles.

Because muscles are structurally in a state of hyper-excitability (because of the biochernical and physiological alteration of muscle control), all kinds of stimuli which can potentially activate muscles are capable of bringing about the more pronounced muscle contractions in CLBP patients. Thus, not only the relevant stressors of Flor et al., but also pain stimuli, startle stimuli, etc. A very potent test would be to bring the muscle to contract by direct electrical stimulation of the muscle. When a change in muscle biochemistry or structure underlies the more pronounced phasic responsivity, contraction by direct stimulation will also be more pronounced in CLBP patients.

Testing of the three last predictions can perhaps also offer further indication as to the extent in which muscular variables play a role in CLBP, by not only looking at mean results, but also at the results for individual patients.

\section{Clinical implications of the model}

The processes speculated to function in the development and maintenance of CLBP could be a basis for setting up clinical trials into the efficacy of a number of specific therapeutic procedures. These studies can at the same time be considered as tests of the clinical predictions of the model. Interventions can be directed at several elements in the model. Postural correction and motor re-education can be applied for the relief of physical strain. It is predicted that these procedures will alleviate pain and that the mechanism of action is through a diminishing of muscle fatigue and of the increased phasic responsivity of lumbar muscles. This can be objectively assessed. However, it might be that the relief of physical strain is most successful in the earlier stages of the pain process. If postural abnormalities have given rise to mechanical irritation, permanent (micro-)damage may have resulted which is irreversible. Nevertheless, even in this stage, when complete recovery may have become impossible, reduction of physical strain could decrease pain intensity or at least prevent exacerbation of the condition.

A possible aid in postural re-education may be EMG-biofeedback. With EMGequipment it can be established in what way muscle activation patterns during movement deviate from the normal patterns in individual patients. After identification of these deviant patterns, a patient can be taught to execute the movement in a more normal way by means of continuous feedback of muscle activity. In a preliminary study (Wolf et al., 1979; Wolf et al., 1982) it was shown that such a procedure was able to reduce the abnormal EMG-pattern which was found during dynamic movement (rotation) in one CLBP patient. Also, the clinical pain level decreased. However, to my knowledge, a more comprehensive study, including more subjects and an adequate control group, has not been performed yet with this kind of feedback.

A second element at which intervention could be directed is mental strain. 
Especially in CLBP patients in which tension appears to be a prominent factor, techniques aimed at reducing stress, or at reducing the impact of stressful situations, will be effective. Several techniques are available: relaxation techniques, stress managements procedures, and more elaborate cognitive therapy. Again, the prediction which can be formulated is not only that these procedures will reduce pain, but also that the underlying mechansims are addressed, and that muscle fatigue and muscle responsiveness will therefore decrease.

The sympathetic involvement which is proposed as a link between stress and the altered muscular control can be a direct aim of intervention. Lumbar sympathetic blockade (by local anesthetics) can be predicted to reduce muscle fatigue, muscle responiveness and pain in CLBP patients. Reduction of pain intensity has been found after lumbar sympathetic blockades in low back pain patients (Connally \& Sanders, 1991). In fibromyalgia patients, regional sympathetic blockade was demonstrated to reduce the fatiguability of muscles in the hand (Backman et al., 1988).

Finally, frequent and profound muscle activation can be directly addressed by paraspinal EMG-biofeedback. So far, most systematic studies into EMG-biofeedback treatment for CLBP have been disappointing. Sometimes there was no reduction of pain, or only an initial clinical improvement which did not remain at follow up. Often, there was no relationship between clinical improvement and change in EMG levels. However, usually biofeedback training has been directed at changing tonic muscle tension levels during relaxed circumstances. This is probably not effective because it was shown that most CLBP patients do not have tonic increases in muscle tension. It can be predicted that procedures aimed at reducing phasic muscle contractions will be more effective. Because stress was shown to be a potent stimulus for inducing phasic muscle contractions, the efficacy of biofeedback during stress stimulation should now be established. Some data are already available pertaining to such a procedure. Flor \& Birbaumer (1991) have employed biofeedback directed at the trapezius and erector spinae muscles, in which "normal" trials and trials with stressful imaginations were alternated. Nineteen patients participated in the biofeedback treatment. Their improvement (pain intensity and interference of pain) was compared to the improvement of an equal number of patients undergoing either an eight-session cognitive behavioural treatment or medical treatment (unspecified). Biofeedback was the most effective treatment, as assessed directly afterwards and also at three months and one-year follow-up. However, Flor \& Birbaumer do not report whether there were actual changes in muscle responsiveness to stress stimuli in these patients. This should be a major aim of future studies.

In the literature, the specificity of the mode of action of biofeedback has been questioned. It is possible that biofeedback addresses mainly non-specific elements. For instance, the feeling of controllability of the pain, might be sufficient to improve the clinical condition. The importance of the feeling of control which is induced by biofeedback was elegantly demonstrated in an experiment by Holroyd et al. (1984). Headache patients received feedback either contingent upon muscle tension decreases ("normal" feedback), or upon muscle tension increases (reverse feedback). Both groups improved on the pain intensity variable after treatment, whether increased or decreased muscle tension levels were obtained. Only the successfulness of the 
feedback seems to have been relevant. Also Biedermann el al. (1987) have shown that it is the non-specific component of biofeedback which induces clinical improvement in the treatment of back pain. These authors suggest that it is the re-education of attributing pain to stress and psychological factors instead of somatic to factors, in combination with relaxation, which causes a diminution of pain. Whether biofeedback directed at phasic muscle contraction has a more specific effect should now be tested.

\section{Cognitive-physiological discordance}

An issue rather distinct from the previous one is related to the observation that the various components (physiological, werbal-cognitive and behavioural) of the pain response in CLBP patients are often discordant. In section 2.6 it was discussed that the most evidence was for discordance between the cognitive and behavioural components of pain. However, the main interest in our own studies was on discordance between the verbal-cognitive and physiological response system, because it has been speculated that this kind of discordance may have an etiological role in several psychophysiological disorders (Gannon \& Haynes, 1986), possibly also in CLBP. In the original hypothesis of Gannon and Haynes it is stated that some people cannot adequately perceive physical sensations, and that they are therefore unable to initiate appropriate coping behaviour. This in turn, predisposes them towards the development of various psychophysiological disorders. It has been discussed previously that this form of discordance is difficult to test in a clinical situation, and that it could perhaps better be tested experimentally by assessing the subjective and physiological responses to an experimental pain stimulus. The advantage of an experimental study over a clinical study is that the nociceptive input can be controlled, and that it is known which physiological responses will be elicited. Two such studies were performed for this dissertation.

In the first discordance study (section 5.4), autonomic responses were measured. It was known beforehand that a certain degree of discordance and desynchrony was to be expected in all subjects, because autonomic responses are not specific for nociceptive stimuli. Therefore, the aim of this study was not to test whether stubjective pain responses (VAS-scores) and autonomic indicators were discordant per se in CLBP patients, but whether discordance and desynchrony would be greater in these patients. This proved not to be the case. There were more or less concordant as well as obviously discondant response patterns between SCR and VAS-scores in all subjects alike. Moreover, the changes in the different responses always displayed a desynchronous course, even between the various autonomic responses themselves.

In the second discordance study (section 5.9), another physiological response was used, namely a muscle-reflex. In this experiment, the co-variation of subjective pain threshold and the RIIIreflex threshold was determined. The RIII-reflex has been shown to be elicited only by stimuli of noxious intensity (Hugon, 1973; Willer, 1979). It is therefore a more specific pain response than any autonomic reaction. Concordance between the subjective pain threshold and the RIII-reflex threshold was expected. This was indeed found, as can be seen from the table of correlations 
between the two thresholds in section 5.9. For control subjects (groupl) and acute pain patients the correlations ranged around 0.80 . However, concordance was much less in CLBP patients, as was shown by the low or even absent correlations. In addition, synchrony of the changes of both thresholds was also less in CLBP patients. The variation in pain threshold within a subject was significantly related to the variation in RIII-threshold only in the control groups and the acute pain group. In CLBP patients the change in pain threshold was not significantly related to the change in RIII-threshold.

If the RIII-reflex is taken as an indicator of spinal nociceptive activity, these data seem consistent with the suggestion that CLBP patients do not accurately perceive nociceptive signals. However, in section 5.4 it was demonstrated that CLBP patients can differentiate between varying levels of intensity of a nociceptive stimulus accurately. In the study reported there, subjective pain intensity scores on the VAS were closely related to stimulus intensity, both in CLBP patients and in controls. On average, there was a correlation of 0.80 between the two variables. This means that $64 \%$ of the variance of the VAS-scores can be explained by the variations in intensity of the pain stimulus. It seems that CLBP patients are capable of perceiving differences in nociceptive signals. Still, in the last experiment, it looks as if the pain reports of CLBP patients are less guided by nociceptive signals than those of control subjects.

The influence of an adaptation level on subjective pain threshold and the much stronger increase of this threshold during the trials do not seem to be sufficient to explain the discordance and desynchrony, for this applied to the acute pain group as well. In this group, discordance was less, and there was no desynchrony. It seems as though the pain reports of CLBP patients fluctuate relatively independently from actual nociception. It can be speculated that cognitive processing of pain signals, or operant learning processes guiding pain report could be responsible fior this. However, it should first be established whether this discordance/desynchrony is a robust phenomenon, and not due to possible statistical artifacts.

In conclusion, discordance and desynchrony between physiological and subjective pain responses in CLBP patients could not be demonstrated when autonomic variables were used as the physiological response. Moreover, it was shown that CLBP patients probably do not have a fundamental inability to perceive nociceptive signals accurately. Nevertheless, increased discordance and desynchrony were found for the relationship between subjective pain threshold and the Rlll-reflex. This finding now awaits confirmation. 


\subsection{Chronic pain and experimental pain sensitivity}

\section{The effect of stimulus intensity on pain sensitivity}

In section 3.8 the preliminary hypothesis that CLBP patients have an increased threshold and decreased tolerance for experimental pain stimuli was formulated. This hypothesis was subsequenty tested in three studies. In the first study (section 5.1), non-significant trends in the predicted direction were found for both variables. The second experiment (section 5.3) did not find a difference between CLBP patients and controls on either pain threshold or pain tolerance. We attributed this to the possibly unreliable method of pain assessment in this experiment.

In the third experiment (section 5.7) the reliability of the pain measurement procedure was improved. With the electrical stimulus, one test trial was given before the actual assessment started, and pain threshold and pain tolerance were derived on the basis of the average of several (ascending and descending) stimulus series, instead of using just one series as before. In addition, a second pain stimulus was added (pressure pain). With this improved design, higher pain thresholds were found in CLBP patients for both stimuli. Contrary to the hypothesis, also a higher pain tolerance was found for CLBP patients. Pressure pain tolerance was significantly higher, and electrical pain tolerance showed a trend towards higher values. Finally, in the last experiment (section 5.9), pain threshold for an electrical shock on the foot was found to be significantly higher in CLBP patients than in controls. No tolerance measurement was performed in this study. The results of the nociceptive reflex assessment will be discussed below.

In sum, the preliminary hypothesis was not integrally supported. Pain threshold indeed appeared to be higher in CLBP patients, in line with the hypothesis, but the findings with pain tolerance were not consistent. In two previous experiments with CLBP patients it was found to be lower (Schmidt \& Brands, 1986; Brands \& Schmidt 1987), in the first experiment of this dissertation there was a trend towards lower values, but in a subsequent experiment, pain tolerance for two different stimuli was higher.

\section{The model}

It was not only tested whether CLBP patients thave different pain threshold and tolerance levels, but also why such effects would occur. In the model presented in section 3.8. it was argued that two opposed mechanisms determine acute pain sensitivity in chronic pain patients. It was proposed that the mechanism responsible for decreased pain sensitivity would be DNIC, or alternatively the adaptation level (AL) mechanism. The pain sensitivity increasing mechanism in chronic pain patients was proposed to be based on hypervigilance in combination with operant learning processes. This mechanism was called a response tendency. It was further suggested that with low intensity pain stimuli, DNIC (or the adaptation phenomenon) would 
predominate, whereas with high intensity stimuli, a response tendency would be predominant. However, on the basis of the findings discussed above, this latter assumption appears to be false: with stimuli at pain tolerance level, increased pain sensitivity was not uniformly found. Therefore, the model as it was originally presented has to be rejected.

However, this does not mean that the operation of two opposed mechanisms in CLBP patients which together determine pain sensitivity is in itself invalid. It does show that the balance between the two mechanisms is not solely dependent on pain stimulus intensity. Other factors seem to be relevant in determining which mechanism will predominate. Which factor(s) this could be will be discussed later in this section.

\section{DNIC versus adaptation level}

In section 3.8. it was argued that a DNIC mechanism better explains the various findings in CLBP patients than an $A L$ mechanism. This concerned the finding that decreased pain sensitivity is especially prominent at pain threshold level (an observation which is still valid, even though it apparently can be found at pain tolerance level too), as well as the finding that CLBP patients have more difficulty in discriminating between stimuli of various intensities. One further objective of our studies was to establish whether postulating a DNIC mechanism is superior to postulating an adaptation level mechanism.

A series of four experiments were set up to test this question. These experiments were aimed at measuring a variable which would not likely be influenced by $A L$, but which would be influenced by DNIC. This variable was pain discrimination in the first two experiments, and RIII-threshold in the last two experiments. For both variables it was first tested whether they could be influenced by an experimental counterirritation stimulus. This proved to be so, and therefore they were used in the subsequent studies comparing CLBP patients with pain-free controls. If the same variables which are affected by an experimental counterirritation stimulus are also affected by chronic pain, a counterirritation effect of chronic pain can be inferred, and DNIC will be the preferred explanation. However, if chronic pain does not mimic this experimental counterirritation effect, the operation of an $A L$ mechanism in chronic pain patients is more likely.

In our first two experiments the effect of experimental and chronic pain on pain discrimination was tested. In a previous study pain discrimination had already been found to be worse during a counterirritation stimulus (Talbot at al., 1989). Also, chronic pain patients were found to be less able to discriminate between pain stimuli of various intensity (Malow et al., 1980; Naliboff et al., 1981; Yang et al., 1985). In fact, this was one of the reasons for preferring DNIC over the adaptation level interpretation. However, replication of these previous findings was important because the pain discrimination task in the experimental counterirritation study by Talbot et al. was not operationalized in the same way as the pain discrimination task on which chronic pain patients were found to perform worse. In the first case a detection task (latency to detect a change in stimulus intensity) was employed, whereas in the latter 
case the SDT (signal detection theory) parameter of discriminability was employed. This SDT parameter of discrimination depended on the subjective rating of pain stimuli, and could have been influenced by response tendencies. Therefore confirmation of this finding with another paradigm not permitting response tendencies to occur was needed. A forced-choice discrimination task was chosen. Before this test was used in the comparison of CLBP patients and controls, it was established whether a lowered discrimination with experimental counterirritation could be demonstrated by means of this paradigm.

In the experiment reported in section 5.6 , counterirritation was induced by means of pressure pain and normal subjects had to perform the pain discrimination task simultaneously. Also, pain threshold and pain tolerance were measured, to test whether the counterirritation effect would be more prominent at pain threshold leve] than at tolerance level. However, contrary to expectation, no counterirritation effect could be demonstrated on either pain threshold or pain tolerance. In the paper we suggested that this could possibly be attributed to the unreliability of the procedure of pain assessment by means of electrical stimulation. This was the second study using this stimulus, in which the results appeared to be inconsistent with previous findings (see the discussion of the experiment of section 5.3 above).

Although no counterirritation effect was found on pain threshold and pain tolerance, in the same study it was shown that in the counterirritation condition pain discrimination was significantly worse than when no counterirritation stimulus was present. The forced-choice discrimination task is probably less sensitive to the strong habituation effects which made electrical stimulation an unreliable indicator of pain threshold and pain tolerance. Thus, the finding that counterirritation has an effect on pain discrimination seems to be also replicable with another experimental paradigm, namely a forced-choice task. However, the alternative explanation that the poorer discrimination was caused by a distraction effect of the pressure pain stimulus cannot be excluded.

Next, the forced-choice pain discrimination task was applied in an experiment comparing CLBP patients with controls (section 5.7). Also, a control task for nonspecific discrimination deficits was added. And of course pain threshold and pain tolerance were measured, this time with a more reliable procedure. It was already discussed above that in this experiment both pain threshold and pain tolerance were significantly higher. However, an impaired ability to discriminate between pain stimuli of different intensity was not found in the CLBP group. Therefore, in this experiment no evidence was found favoring the DNIC interpretation over AL. Yet, this study was not conclusive as to the question of whether chronic pain is able to activate DNIC, because the finding of poorer discrimination in the previous study could have been based on the alternative interpretation of distraction. It has not definitively been established that counterirritation can actually influence such a forced-choice task. Thus, a more conclusive experiment had to be designed to find out whether chronic pain does or does not active DNIC.

The technique of RIII-reflex measurement was of great value in this context. DNIC, or counteritritation, had already several times been shown to be able to depress the RIII-reflex, whereas a perceptual phenomenon like AL can hardly be 
expected to have an effect on spinal reflexes. In previous studies, depression of the RIII-reflex by counterirritation was found with one kind of pain stimulus: the immersion of a hand in painfully hot water. Therefore it was first verified whether this finding could be generalized to other counterirritation stimuli as well. A study was set up to test the efficacy of two different stimuli: an ischemic pain stimulus and the cold pressor test. In section 5.8 it was reported that both stimuli were effective in reducing the magnitude of the RIII-reflex.

The design of the last study (section 5.9) allowed for a definite conclusion concerning the question of whether the DNIC interpretation is preferable to $\mathrm{AL}$. It was tested whether chronic pain would not only increase subjective pain threshold, which can be explained by both DNIC and AL, but also the RIII-reflex. The latter cannot easily be explained by AL. Moreover, we made use of the fact that DNIC was found to be antagonized by naloxone. If the increased pain threshold in CLBP patients is determined by DNIC, naloxone will reverse this. Finally, in order to exclude the eventual alternative interpretation that nociceptive inhibitory processes result from the psychological morbidity often seen in chronic pain patients (depression, chronic anxiety or stress) and not from pain per se, an acute pain group was also included in the experiment. In this group, nociceptive activity was pertinent, whereas there were no psychological or other changes as a result of longstanding pain.

The study demonstrated that chronic back pain does not activate DNIC. The spinal RIII-threshold was not higher in CLBP patients, and naloxone did not have any effect on pain threshold or RIII-threshold in the patient group. Consistent with other studies, subjective pain threshold was again higher for CLBP patients. Therefore, it can be concluded that the adaptation level theory seems after all to be a more likely explanation for the decreased pain sensitivity in chronic pain patients than the operation of DNIC.

\section{Response tendencies}

Another issue, which has only been addressed briefly, relates to the process of increased pain responsivity as a result of response tendencies. Is there any evidence to assume that CLBP patients display such response tendencies? The occurrence of one kind of response tendency was tested in the study reported in section 5.3 . In this study, a response tendency was defined as the report of a sensation when low intensity electrical shock was suggested but not actually delivered. No evidence was found that CLBP patients were more often inclined to report a sensation than control subjects. However, this finding cannot be very conclusive as to the occurrence of response tendencies in general, because of the specific way in which the tendency was defined here. Some individuals might be inclined to complain excessivelly when just minor discomforts are present, but this does not mean that they will "complain" of sensations when there is actually no exteroceptive stimulation. In the same experiment, it was shown that CLBP patients did report more non-specific bodily sensations on the somatization subscale of the NVM (short Dutch version of the MMPI). It seems as though there was a response tendency to report more clinical sensations, 
ether because these patients are more sensitive to these sensations (hypervigilance), or because they are more inclined to complain about such sensations (operant learning process).

Thus, whether and under what conditions response tendencies can determine the pain responses of chronic pain patients has not been resolved yet.

\section{Other factors determining pain sensitivity}

If was demonstrated that stimulus intensity cannot be the only factor which determines whether decreased versus increased pain responsivity is found, and one or more other factors have to be postulated. A factor which may be of primary importance could be the duration of pain stimulation. Pain tolerance measures are usually tests of endurance, thus the pain stimulus is present for a relatively long time. Whereas at first a subject's pain response might be determined mainly by actual pain perception, if the pain persists, psychological processes become more important. Subjects probably become annoyed by the pain, and at some moment in time have to make a cognitive decision when to stop the stimulus. Especially with pain tests where the increase in intensity is only slow, or even more extreme, levells off after a while, the decision will be based more on psychological processes than on actual pain perception. In CLBP patients actual pain perception will be determined in part by AL, whereas the conscious decision is more powerfully affected by the results of learning processes they have been going through (increased complaining, learned helplessness, avoidance, etc). Moreover, cognitive elaboration of the pain stimulus and coping strategies will also occur, especially in endurance tests. It can be speculated that CLBP patients have more negative cognitions related to pain (e.g. low sense of control, low self-efficacy beliefs; Philips, 1987; Flor \& Turk, 1988), and use less effective pain coping strategies (Slade et al., 1983). An effect of cognitions and coping strategies on experimental pain has been demonstrated previously (eg. Spanos et al., 1979).

This hypothetical interpretation is consistent with our results. With pain threshold tests, evidence for $A L$ is found. Pain tolerance, measured by means of electrical stimuli, has also found a trend towards decreased pain sensitivity, thus favoring AL. In our studies, electrical shock was the only pain tolerance stimulus which did not rely on endurance. Discrete short stimuli were presented in series. Thus, with every stimulus, pain was only present for a minimal amount of time and the sensation was well-defined. In contrast, the CP test which was used by Schmidt and Brands was a real endurance test. The maximum duration of the stimulus was five minutes. Often, it was observed that pain did not increase any more after a while: the hand became numb. The decision when to report pain tolerance therefore had to rely on other factors than pain perception alone. With this test, lower pain tolerance was reported for CLBP patients in two successive studies. Finally, another test which has been used was the pressure pain test. This pain stimulus lasted maximally three minutes, and it can again be considered a test of endurance. In the first experiment with this stimulus, a trend towards lower pain tolerance was found in CLBP patients, whereas 
in the second experiment with this stimulus higher pain tolerance was found. The difference between the two studies was the absolute pressure which was applied to the finger. In the first study the weight was much lighter than in the second study, and it took considerably more time to reach pain tolerance. For control subjects this was on average $138 \mathrm{sec}$. in the first study. In the second study, the weight was made heavier and controls reached pain tolerance in only $46 \mathrm{sec}$ on average. It can be suggested that with the long stimulus duration and the gradual pain increase in the first test, there was more opportunity for psychological factors to become activated and to influence the decision process than in the second test.

The above speculation can be tested in an experimental study. The same subjects, CLBP patients and controls, should be tested with two different pain tolerance tests, one a test of endurance, the other employing discrete well-defined stimuli. Electrical pain probably qualifies best as the stimulus for such an experiment because shocks can be delivered in discrete steps, or electrical current can be delivered continuously, with a steady and gradual increase in intensity. If the speculation above is valid, it would be expected that in the discrete stimuli test, CLBP patients have a higher pain tolerance. However, with steady and gradually increasing electrical stinulus intensity, and the need for a conscious decision when to stop the stimulus, CLBP patients would have a lower pain tolerance. This research could be taken one step further, by experimentally manipulating the coping strategies of subjects. Two groups of normal controls could be taught different coping strategies (e.g. focus of attention on the stimulus vs. distraction from the stimulus), and subsequently subjected to the two different pain tolerance tests described above. It can be predicted that the subjects who have learned an effective coping strategy (distraction) perform better in the endurance test, but that there is no difference in the test measuring pain tolerance by means of discrete pain stimuli.

Thus, in addition to stimulus intensity, a second important factor in determining pain sensitivity in chronic pain patients could be stimulus duration, and this should now be tested further. But still other factors could be implicated in the regulation of pain sensitivity too. Recently, Naliboff \& Cohen (1989) have even proposed five factors which might determine the ultimate pain responsivity of chronic pain patients. The first factor was resemblance of the experimental stimulus to clinical pain, with stimuli with close resemblance leading towards increased pain sensitivity. The second factor is the quality of the pain stimulus. Stimuli which are affectively coloured would elicit a pattern of hypersensitivity, whereas sensory/discriminative stimuli would probably elicit a pattern of hypo-sensitivity. The type of response which is required of the subject was the third factor. A behavioural response would predispose towards over-responsiveness, simple pain reports would predispose towards under-responsiveness. The mood of the subject was factor number four. More anxious people would be more sensitive, depressed people less sensitive. Finally, the last factor which was named is similar to the preliminary hypothesis in this dissertation: intensity. Intense stimuli could predispose towards increased sensitivity, mild stimuli towards decreased sensitivity.

A systematic test of these factors has not been performed. But at least one of the factors can seriously be questioned: mood. The effect of anxiety is not always an in- 
crease in pain sensitivity. Experimental research has shown that only anxiety directed al the pain stimulus itself increases sensitivity, whereas irrelevant anxiety decreases sensitivity instead (Amtz et al., 1991; Al Absi \& Rokke, 1991). But more importantly, the effect of depression in general seems to be to increase pain responsivity (pain rating and pain tolerance, Philips \& Jahanshahi; Amtz et al., 1991; Zelman et all, 1991), and not to decrease pain responsivity as is suggested by Naliboff and Cohen. The validity of the other factors should be examined in a more systematic way.

\section{Sensitivity to non-painful signals}

Sensitivity to non-painful tactile stimuli has been tested in two of our studies. In the study reported in section 5.3 detection threshold (DT) for an electrical stimulus was measured. In the next study (section 5.5) tactile two-point discrimination threshold was assessed. Most of the processes speculated to play a role in CLBP do not specifically predict a difference in tactile sensitivity. Adaptation level only concerns the judgement and perception of painful stimuli. Operant conditioning can possibly manifest itself as increased complaining of both painful and non-painful sensations, but this will not influence a detection/discrimination task as is employed here. DNIC can perhaps lower tactile sensitivity. In the theoretical section it was described that DNIC inhibits all activity of convergent neurons, that is, also A- $\alpha$ fiber activity. However, it has never actually been demonstrated that a counterirritation stimulus was able to affect detection threshold. In our first counterimitation experiment (section 5.6) a pressure pain stimulus had no effect on stimulus detection. Moreover, because now it has been shown that chronic pain does not activate DNIC, this mechanism can be discarded as a possible cause for differences in tactile sensitivity.

Hypervigilance seems to be the only mechanism which could influence tactile sensitivity in chronic pain patients. Subjects especially attentive to all kinds of bodily sensations might have a lower detection threshold for physical stimuli in general. Possibly they even perform better in a two-point discrimination task.

The data showed that there was no evidence for a lower detection threshold for electrical stimuli in CLBP patients. In fact, DT even appeared to be higher in the patient group as compared to controls, although this difference did not reach statistical significance. In a previous study, DT for heat stimuli had been found to be similar for CLBP patients and controls (Naliboff et al., 1981). In any case, no evidence for a hypervigilance mechanism was found. Tactile two-point discrimination did not differ between CLBP patients and controls either.

\section{An alternative model of experimental pain sensitivity in chronic pain patients}

On the basis of the various studies, it has to be concluded that the model which was formulated to explain pain sensitivity in chronic pain patients in general and CLBP patients more specifically, should be rejected. Increased versus decreased pain 
sensitivity was not solely dependent on stimulus intensity. Moreover, the mechanism which was proposed to be responsible for decreases in pain sensitivity, DNIC, was shown not to become activated by chronic pain.

An alternative model of pain sensitivity in CLBP patients can be proposed. The assumption that the operation of two opposed factors determines the final pain responsivity can be maintained. The pain decreasing mechanism is most likely to be based on an adaptation level phenomenon. The identity of the pain increasing process is still speculative: either hypervigilance, operant learning processes, cognitive processes or a combination of these. There are two findings which make hypervigilance less likely than operant and cognitive learning processes: CLBP patients are not better in detecting low intensity physical signals and they seem to be less accurate in perceiving nociceptive activity as shown by the low correlations between RIII-reflex and pain threshold (see section 5.9). Therefore, operant and cognitive learning processes seem to be the preferred interpretation for the increased pain responsivity, but this should be tested further.

Which of the two opposed processes will be the most important at a certain moment in a particular patient varies. It can be speculated that with longer lasting endurance tests in which pain increases gradually, the pain increasing mechanism (learning influences) will predominate. However, with phasic pain stimuli and pain rating tasks, AL might predominate. Other factors could also influence the ultimate pain responsivity of chronic pain patients, and these should be investigated further.

\subsection{Chronic pain and the habituation to acute pain stimuli}

In the last part of this discussion, the results of the two studies measuring habituation of CLBP patients to experimental pain stimuli will be reviewed. On the basis of the findings of a study by Brands \& Schmidt (1987) in which learning influences on pain tolerance were found in control subjects but not in CLBP patients, the hypothesis was formulated that a fundamental deficit in habituation to painful experiences could be a risk factor for developing CLBP. It was argued that not getting used to a constant source of nociceptive input can predispose a subject to sustained pain behaviours (postural adaptations, avoidance of activity). In the first part of the discussion it was speculated that such behaviours can be very detrimental for rehabilitation and can exacerbate or sustain the pain complaints.

However, in chapter 4 of this dissertation it was concluded that CLBP patients probably do not have a fundamental deficit in habituation to pain stimuli. In a study of Arntz et al. (1991) it was shown that both the subjective responses (VAS-scores) and the physiological responses to a series of 20 electrical pain stimuli showed the same decreasing trends in back pain patients and controls. It was speculated that differences between CLBP patients and controls in habituation to pain stimuli may be found in certain circumstances, but that these differences have to be ascribed to factors other than deficient habituation capacity. On the basis of two additional studies, it 
will now be argued that differences in habituation do not distinguish between CLBP patients and controls but that other more complex learning patterns (cognitive or operant) may be involved.

In the first habituation study three subjective parameters were assessed (pain threshold, pain tolerance and VAS-scores at an intermediate stimulus intensity) and several physiological variables. The design and results of this study are presented in detail in sections 5.1 and 5.2 , for the subjective and physiological data respectively. In short, it appeared that the habituation of none of the physiological variables which were measured differentiated between CLBP patients and controls, confirming the results of the Arntz et al. study. It should be noted, thowever, that most variables did not habituate at all, in either group. Yet, the one variable which did habituate (number of SCR's) did so in the two groups to an equal degree.

Remarkably, in the same study different learning processes were found for at least one of the subjective variables. There was clear evidence that the pain threshold of CLBP patients did not increase after repeated trials, whereas it did increase in controls. Regarding pain tolerance, no definite conclusion could be drawn, but there was some indication that this response also showed an increase in the control group but not in the patient group. VAS pain ratings did not systematically change in either group $p^{2}$.

In our last experiment, changes in subjective pain threshold and the RIII-reflex threshold were determined in 9 successive assessment periods. Unlike the previous studies, the actual experiment was preceded by a training period which already eliminated part of the habituation. Yet, the RIII-reflex threshold still increased slightly, and the subjective pain threshold increased even quite substantially still. In this study, no evidence for impaired habituation in CLBP patients was found for either variable. The remaining habituation of the RIII-reflex was similar for CLBP patients and controls. Surprisingly, the subjective pain threshold increased even more in the patient group compared to that in the control group.

In sum, our two studies supported the conclusion which was made in section 4.5 that CLBP patients do not have a fundamental deficit in habituation to pain stimuli in general. They are capable of habituating to repeated painful stimulation on both subjective and physiological levels, and therefore the differences which were found for some variables have to be expllained in another way. An important observation is that differences were only found for the changes in subjective and behavioural variables. Diminished habituation of any of the physiological variables in the various experiments was never demonstrated. Therefore, perhaps a distinction should be made between the different learning processes which can operate on the various responses.

Autonomic reactions and motor-reflexes (e.g. the RIII-reflex) can be considered true unconditioned responses (UCR's), which are elicited by an unconditioned stimulus (UCS, a nociceptive stimulus in this case). Two learning processes can affect the UCS-UCR association. First, by respondent conditioning a previously uncondi-

2 This can possibly be atributed to the fact that for this variable only 6 scores were avalable, instead of 8 . During the first and last pain threshold and tolerance assessments no pain ratings were made (see for a discussion of this topic section 5.1 ). 
tioned response can be coupled to a new stimulus (the conditioned stimulus) and thereby become a conditioned response. But learning can also affect the UCR itself, especially its magnitude. This magnitude will obviously be determined by the intensity of the stimulus, but as has extensively been discussed in chapter 4 , response magnitude can be modified by habituation or sensitization. These are probably the most important learning processes at this level of responding. The operation of more complex learning processes (cognitive and operant) on autonomic and motor-reflex responses seems to be possible (van Olst et al. 1980). In animal research it was demonstrated that autonomic responses can in some circumstances be operantly conditioned (Miller, 1969). However, at this level of responding, operant and cognitive learning is probably far less important than the more simple process of habituation and sensitization.

In contrast to the autonomic and motor-reflex responses, the subjective pain experience is subject to numerous influencing factors. Several of these factors were included in the schematical representation of pain experience in the introduction. It can be assumed that modification of pain experience will not only depend on the primitive learning processes of habituation and sensitization, but also on additional more complex processes (cognitive and operant learning). Another complicating factor is the fact that pain experience is not directly accessible for observation, and the experimenter necessarily has to rely on verbal pain report and pain behaviour, variables which in themselves are subjected to all kinds of learning processes.

In conclusion, decreases in the magnitude of autonomic variables and the RIIIreflex can probably be ascribed to habituation. By contrast, decreases in subjective pain reports cannot be ascribed to habituation exclusively. The formation of new pain cognitions, the extinction of previously established behaviour patterns and the establishment of new pain behaviours will all play a role in the final pain responsivity. No differences between CLBP patients and controls were found for the decrease in magnitude of autonomic variables and the RIII-reflex, the responses almost exclusively subjected to habituation, but differences were found for the decrease of subjective pain variables, which are influenced by several learning processes. The most obvious conclusion therefore is that different change patterns in CLBP patients are probably not due to differences in habituation but to the influence of other forms of learning. Exactly how this works cannot be resolved at this moment. Yet, it is possible to speculate about the learning processes involved. Two possibilities will be presented.

First, the diminished decrease of subjective pain responses in CLBP patients could be related to an absence of extinction of previously learned behaviour. Whereas the subjective pain response of control subjects could be largely determined by actual pain experience and the decreases to habituation of nociceptive responses, CLBP patients may be inclined to respond in a previously established response pattern, characterized by increased complaining and avoidance of high pain levels." A few

\footnotetext{
The importance of avoidance of potentially (clinically) painful situations in CLBP patients has been stressed by Philips 11987 ). It has also been speculated that CLBP patients display fear of pain, leading to avoidance (Lethem et al., 1983) This could generalize to the laboratory situation.
} 
experimental trials will probably not be sufficient to extinguish this previously established behaviour. In the two studies in which less change of pain tolerance was found, only 8 trials were given. Moreover, an additional factor probably hindering extinction in these experiments was the possibility for the subject to keep on avoiding high pain levels. The stimulus was withdrawn when pain tolerance was reported. Sustained avoidance (by reporting pain tolerance) prevents the occurrence of extinction. In the two other experiments in which no differences were found, inescapable electrical shocks were giwen. An experiment which might be relevant in this context, was conducted by Philips \& Jahanshahi (1985). Migraine patients who were exposed to inescapable aversive auditory stimuli (stimuli they usually avoid), increased their tolerance for these stimuli on a second occasion, whereas patients who could avoid exposure to these tones, had decreased tolerance on a second occasion.

The second possibility is that the differences which have been found in learning are cognitively based. After repeated (harmless) pain stimulation a subject will normally change his/her cognitions about this stimulus. When this subject learns that (s)he can cope effectively with the pain, beliefs of self-efficacy increase. In the previous section it was speculated that CLBP patients have more negative cognitions about harmfulness of pain, and beliefs of low selfeefficacy to endure pain. These beliefs could be relatively difficult to change, because they are firmly incorporated into the cognitive structure of the patient. Coping and beliefs of self-efficacy will especially be important in pain endurance tests. In turn, the poor endurance of CLBP patients in these tests may increase negative cognitions and the bellef of low self-efficacy even more.

There are obvious clinical implications of both explanations for the differences of learning processes. If patients do not engage in potential painful activities because of low self-efficacy beliefs and the negative interpretation of pain, this could be a starting-point for therapeutic intervention. The implication of the explanation of sustained avoidance behaviour was already elaborated by Philips (1987). Therapy could be aimed at identifying the situations which are avoided by CLBP patients, followed by exposure training to overcome this avoidance. However, more research is needed to identify whether operant or cognitive aspects (or both) are implicated in maintaining pain behaviour.

In sum, several research findings point in the direction of specific differences in the learning processes which CLBP patients undergo when confronted with repeated (experimental) pain stimuli. Probably the most fundamental learning process (habituation) is not "defective" in CLBP patients. It is more probably that this group is characterized by an inflexibility in changing their pain responses because of firmly established beliefs of low self-efficacy. It is also possible that because of sustained avoidance of pain stimulation, extinction of previously learned behawiour patterns does not occur in CLBP patients. 


\subsection{Concluding remarks}

Various issues relating to chronic pain and pain sensitivity have been addressed in this dissertation. The experimental studies of chapter 5 have provided an answer to some of the questions which were raised in the theoretical section, but these studies also generated a host of new research questions. In this last chapter, several suggestions for further research have been made. An important future research issue would be to elucidate the role of muscular variables in the maintenance of low back pain. The model presented in section 6.1 could serve as a theoretical framework. The therapeutical implications of this model are apparent.

The research on experimental pain sensitivity has suggested the importance of judgmental (adaptation level), cognitive and operant variables on pain perception. Differences in pain responsivity between CLBP patients and controls can possibly be ascribed to prior established differences in cognitive structures. Identification of the most relevant factors influencing pain perception, in experimental as well as in clinical situations, should be a major aim in future studies. Fortunately, in recent years the importance of cognitive factors in chronic and acute pain perception has been recognized. Therapeutic interventions based on the cognitive approach have begun to develop. Experimental laboratory research can be a useful addition to clinical studies. 

This dissertation deals with the perception of experimental and clinical pain by chronic pain patients. In six chapters, several aspects of this broad area are highlighted. In the first chapter a general introduction to the research area is provided and the concepts of "pain" and "chronic pain" as they are used in this dissertation, are defined. Next, three theoretical chapters are presented, in which three different questions pertaining to chronic pain and pain perception are discussed.

In chapter two, the question of causal and contributing psychophysiological factors in the experience of various unexplained chronic pain conditions (specifically back pain and headache) is addressed. The three most influential psychophysiological models are introduced and research relating to these models is presented. The models which will be addressed are the reflex-spasm model, the stress-reaction model and the biomechanical model. In addition, the concept of cognitive-physiological discordance is considered.

In chapter three, another approach to the issue of chronic pain and pain perception is chosen. The discussion in this chapter centers around the question of whether chronic pain patients have a different perception of experimental pain stimuli. Several hypotheses can be advanced which predict such differential pain perception in chronic pain patients. The various models and theories relating to this issue are reviewed. Next, an exhaustive overview of studies testing the relationship between chronic pain and experimental pain sensitivity is presented, culminating into a preliminary descriptive hypothesis. This hypothesis boils down to the assumption that chronic pain patients are hyposensitive to low intensity pain stimuli, whereas they are hypersensitive to high intensity pain stimuli. Finally, a new and tentative model of pain sensitivity in chronic pain patients is presented, in which the simultaneous operation of two opposed mechanisms is proposed. The respective mechanisms are response tendencies, increasing pain sensitivity, and diffuse noxious inhibitory controls (DNIC), decreasing pain sensitivity.

The last theoretical chapter (chapter 4) deals with habituation to repeated pain stimulation. The core question of this chapter is whether chronic pain patients show a different pattern of habituation to experimental pain stimuli. First, three models of habituation in general are discussed. Next, evidence is presented that humans are capable of habituating to painful stimuli, and factors influencing the rate and degree of habituation of pain responses are discussed. Finally, the scant data pertaining to habituation to pain stimuli in chronic pain patients which is already available is reviewed. It is concluded that specific differences might exist between chronic pain patients and painffree volunteers in the habituation to experimental pain stimuli, but that there is no fundamental habituation deficit in chronic pain patients.

Chapter 5 presents the experimental studies which have been performed for this dissertation. In nine studies, several of the questions raised in the previous theoretical chapters are examined. Psychophysiological models of chronic pain are addressed in only one of these studies. Some evidence is found for the biomechanical model of 
chronic back pain.

The emphasis of most of the empirical studies, was on testing the model of experimental pain sensitivity in chronic pain patients. Several of these studies have tested whether differences between chronic back pain patients and pain-free controls could be demonstrated in either pain threshold, pain tolerance, pain discrimination or pain intensity ratings. A number of differences between the groups was indeed revealed, but the data were not consistent with the preliminary hypothesis which was formulated in chapter 3. In addition, the plausibillity of the two proposed mechanisms was examined. Especially the DNIC mechanism was addressed. No evidence for the operation of this pain-inhibiting mechanism in chronic back pain patients was found.

Finally, in three of the empirical studies, subjective and psychophysiological habituation of pain responses in chronic back pain patients is compared to the habituation in pain-free subjects. Differences were found on a subjective level only.

In chapter 6 an attempt is made towards reaching a general conclusion concerning the three major research topics. In relation to psychophysiological models of chronic pain, it is concluded that there seems to be evidence for the biomechanical model of chronic back pain. However, other recent studies have been supportive of the stressreaction model, and in particular for an elaboration of this model, the diathesis-stress model. Therefore, an integrated view of back pain and contributing psychophysiological factors is presented, incorporating elements of both the above-mentioned models. On the basis of this model, new research can be generated.

Several of the studies which were presented in chapter 5 have tested the tentative model of experimental pain perception by chronic pain patients. The combined results of the individual studies demonstrate that the model should be rejected. Whether increased or decreased experimental pain sensitivity is found in chronic pain patients does not onlly depend on the intensity of the pain stimulus. It is proposed that additional factors are critical too. Moreover, DNIC does not seem to be the mechanism responsible for the (sometimes) decreased pain sensitivity in chronic pain patients. A mechanism based upon the adaptation level theory can alternatively be proposed.

In the last part of the discussion, the results of the habituation studies are reviewed. It is concluded that chronic low back pain patients do not have a fundamental deficit in habituation to painful experiences. The differences in pain responsivity which were found between patients and controls after stimulus repetition, should probably be as cribed to differences in more complex learning processes. The way in which an individual responds to pain will be determined in part by operant and cognitive learning factors. Well-established response patterns are presumably not changed easily by the only few repetitions of an experimental pain stimulus. Especially in chronic pain patients in whom response patterns are particularly well learned and firmly established, a change could be difficult to bring about. 


\section{SAMENVATTING}

Dit proefschrift heeft als onderwerp de perceptic van experimentele en klinische pijn door chronische pijnpatiënten. In zes achtereenvolgende hoofdstukken worden verschillende invalshoeken van dit brede onderzoeksterrein behandeld. Het eerste hoofdstuk presenteert een algemene inleiding, en de begrippen "pijn" en "chronische pijn" worden nader gedefinièerd. Vervolgens worden in drie theoretische hoofdstukken verschillende vraagstellingen met betrekking chronische pijn en pijnperceptie verder uitgewerkt.

Hoofdstuk 2 behandelt de rol die psychofysiologische factoren kunnen spellen in de ervaring van verschillende onverklaarde chronische pijnklachten, met name bij rugpijn en bij hoofdpijn. De drie meest invloedrijke psychofysiologische modellen worden geïntroduceerd, en het onderzoek met betrekking tot deze modellen wordt besproken. Aan bod komen het reflex-spasme model, het stress-reactie model en het biomechanische model. Bovendien wordt het begrip cognitieve-fysiologische discordantie besproken.

In hoofdstuk 3 wordt het onderwerp chronische pijn en pijnperceptie op een andere manier benaderd. De vraag die in dit hoofdstuk aan de orde komt is of chronische pijnpatiēnten een afwijkende gewaarwording voor experimentele pijnprikkels hebben. Eerst wordt een aantal theorieën en modellen besproken die een dergelijke afwijkende pijngewaarwording kunnen verklaren. Vervolgens wordt een volledig overzicht gegeven van studies die de relatie tussen chronische pijn en experimentele pijngevoeligheid hebben onderzocht. Op basis van dit overzicht wordt een voorlopige hypothese opgesteld die de relatie tussen chronische pijn en experimentele pijn beschrijft. Deze hypothese komt erop neer dat chronische pijnpatiënten minder gevoelig zijn voor zwakke experimentele pijnprikkels terwijl ze juist gevoeliger zijn voor sterke pijnprikkels. Dit uitgangspunt vormt tevens de basis voor het model dat in hetzelfd hoofdstuk gepresenteerd wordt en dat verondersteld dat de uiteindelijke pijngewoeligheid van chronische pijnpatiënten afhankelijk is van de gelijktijdige werking van twee aan elkaar tegengestelde mechanismen. Het ene mechanisme verhoogt de pijngevoeligheid terwijl het andere mechanisme de pijngevoeligheid doet afnemen. Het pijn-werhogend mechanisme zou gewormd kumnen worden door respons tendenties die in chronische pijnpatienten optreden, en die gebasseerd zijn op aangeleerd pijngedrag enerzijds en overdreven aandacht voor lichamelijke signalen anderzijds. Het neurofysiologische mechanisme "diffuse noxious inhibitory controls" (DNIC) zou verantwoordelijk kunnen zijn voor het pijn-reducerende proces. DNIC kan kortweg omschreven worden als het verschijnsel dat de ene pijn de andere pijn remt. In het geval van chronische pijnpatiënten zou de klinische pijn een remmende werking kunnen hebben op de ervaring van experimentele pijnprikkels.

Het laatste theoretische hoofdstuk (hoofdstuk 4) behandelt habituatie aan pijnprikkels. De centrale vraag in dit hoofdstuk is of chronische pijnpatiënten een afwijkend leerpatroon bij herhaling van pijnprikkels vertonen: verloopt de gewenning aan pijn trager? Het hoofdstuk begint met de presentatie van drie algemene habituatie 
modellen. Daarna wordt een aantal factoren besproken die de mate en snelheid wan habituatie van pijnresponsen kunnen beïnvloeden. Tenslotte worden de weinige gegevens die reeds beschikbaar zijn op het gebied van habituatie aan pijnprikkels bij chronische pijnpatienten op een rijte gezet. De voorlopige conclusie op basis van dit onderzoek luidt dat mogelijk wel specifieke verschillen bestaan tussen chronische pijnpatiênten en mensen zonder pijn, maar dat er geen sprake is van een fundamenteel habituatie deficiet in chronische pijnpatiënten.

Na de drie theoretische hoodstukken worden in hoofstuk 5 de experimentele studies die voor dit proefschrift zijn verricht gepresenteerd. In negen studies zijn verschillende wan de vragen die opgeworpen zijn in de theoretische hoofstukken nader onderzocht. Hoewel de meeste onderzoeksvragen betrekking hadden op chronische pijn in het algemeen, zijn uitsluitend mensen met chronische lage rugpijn voor deelname aan de studies geselecteerd. Eén van de studies heeft gekeken naar de psychofysiologische responsiviteit van chronische rugpijnpatiènten op experimentele pijnprikkels. De resultaten van deze studie waren hei meest in overeenstemming met de voorspellingen uit het biomechanische model van rugpijn.

De nadruk van de empirische studies lag echter op het testen van het in hoofdstuk 3 gepresenteerde model voor experimentele pijngevoeligheid in chronische pijnpatiënten. In verschillende studies is getoetst of er een verschil kon worden aangetoond tussen chronische rugpijnpatiënten enerzijds en controles anderzijds met betrekking tot pijngevoeligheid. Inderdaad werd op een aantal variabelen een verschil tussen de twee groepen gevonden, maar de resultaten waren niet in overeenstemming met de eerder genoemde hypothese dat de patiënten gevoeliger zouden zijn voor sterke prikkels en minder gevoelig voor zwakke prikkels. Ook de houdbaarheid van de twee veronderstelde tegengestelde mechanismen in het model is onderzocht. De nadruk lag op het uitzocken of chronische pijn het DNIC mechanisme zou kunnen activeren. Hierwoor zijn geen aanwijzingen gevonden.

Tenslotte is in drie studies de subjectieve en psychofysiologische habituatie van pijnresponsen bij chronische rugpijnpatiënten vergeleken met die bij pijnvrije proefpersonen. In een eerste studie bleek de drempel voor drukpijnstimuli minder snel toe te nemen bij pijnpatienten dan bij controles na acht herhalingen van de test. Echter, in de andere studies werden geen verschillen gevonden tussen controles en pijnpatienten op de psychofysiologische of subjectieve pijnvariabelen.

In hoofdstuk 6 is getracht om tot een algemene conclusie omtrent de drie belangrijkste onderzoekswragen te komen. Met betrekking tot de psychofysiologische modellen van chronische pijn kan geconcludeerd worden dat er aanwijzingen zijn voor het biomechanische model. Echter, ander recent onderzoek heeft steun gevonden voor het stress-reactie model. Een geintegreerde visie op de psychofysiologie van rugpijn wordt tenslotte woorgesteld die elementen van beide bovengenoemde modellen combineert. Dit model veronderstelt dat zowel fysieke als mentale belasting een risicofactor voor het ontwikkelen van pijnklachten zouden kunnen vormen. Op basis van dit model worden een aantal nieuwe onderzoeksvragen gegenereerd.

De algemene conclusie van de studies die de pijngevoeligheid van rugpijnpatiënten voor experimentele pijnprikkels hebben gemeten luidt dat het in hoofdstuk drie voorgestelde model niet houdbaar is. Of toegenomen dan wel afgenomen pijngevoelig- 
heid wordt gevonden in chronische pijnpatiënten is niet uitsluitend afhankelijk van de intensiteit van de pijnprikkel. Waarschijnlijk spelen tevens andere factoren hierbij een rol, zoals de duur van de pijnprikkel. Bovendien wijzen de studies uit dat DNIC waarschijnlijk niet het mechanisme is dat een afgenomen pijngevoeligheid in chronische pijnpatiënten kan verklaren. Als alternatief wordt een meer psychologisch mechanisme voorgesteld, namelijk het "adaptation level" fenomeen. Dit veronderstelt dat pijnpatiënten minder gevoelig zijn voor experimentele pijnprikkels oindat ze deze prikkels in relatie tot hun eigen pijn beoordelen.

In het laatste deel van de discussie worden de resultaten van de habituatie studies besproken. Geconcludeerd kan worden dat chronische rugpijnpatiënten geen fundamentele afwijking hebben met betrekking tot habituatie aan pijnlijke ervaringen. De verschillen die zijn gevonden tussen pijnpatiënten en controles na herhaalde pijnstimulatie moeten waarschijnlijk toegeschreven worden aan meer complexe leerprocessen. De manier waarop iemand reageert op pijn zal deels bepaald zijn door operante en cognitieve leerfactoren. Aangeleerde responspatronen zijn waarschijnlijk niet makkelijk te veranderen door slechts enkele herhalingen van een experimentele pijnprikkel. Juist in chronische pijnpatiënten bij wie responspatronen extra stevig verankerd zijn is het waarschijnlijk moeilijk om een dergelijke verandering teweeg te brengen. 



\section{REFERENCES}

Ahern, D., Follick, M., Councill, J., Laser-Wolston, N. \& Litchman, D. (1988). Comparison of lumbar paravertebral EMG pattern in chronic low back pain patients and non-patient controls, Pain, 34: 153-160.

Ahles, T., Cassens, H. \& Stalling, R., (1987). Private body consciowsmess, anxiety and the perception of pain, Journal of Behavior Therapy and Experimental Psychiatry, 18: $215-222$

Al Absi, M. \& Rokke, P. (1991). Can anxiery thelp us tolerate pain?, Pain, 46: 53-52.

Almay, B., Johansson, F., Von Knorning, L., Terenius, L. \& Wahlstrom, A., (1978). Enciorphins in chronic pain. 1. Differences in CSF endorphin levels between organic and psychogenic pain syndromes, Pain, 5: 153-162.

American Medical Association, (1988). Guides to the evaluation of permanent impairnent, 3ed.

American Psychiatric Association, (1987). DSM-I/t-r, Washingiton.

Anderson, K., Pearl, G. \& Honeycutt, C., (1976). Behavioral evidence showing the predominance of diffuse pain stimuli over discrete stimuli in influencing perception, Journal of Neuroscience Research, 2: 283-289.

Anton, F., Kreh, A., Reeh, P. \& Handwerker, H., (1984). Algesimetry using natural stimuli of long duration, In: Biromm. B., Pain Measurement in Man. Neurophysiological Correlates of Pain, Amsterdam: Elsevier, 455-461.

Arena, J., Blanchard, E., Andrasik, F. A Appelbaum, K. \& Myers, P.. 11985). Psychophysiologic comparsions of three kinds of headache subjects during and between headache states, Journal of Psychosomatic Research, 29: 427-441.

Arena, J., Hannan, S., Bruno, G., Smith, J. \& Meador, K., (1991). Effect of movement and position on muscle activity in tension headache sufferers during and between headaches, Journal of Psychosomatic Research, 35: 187-195.

Arena, $\mathbb{J}$., Sherman, R., Bruno, G. \& Young, T., (1991b). Electromyographic recordings of low back pain subjects and non-pain controls in six different positions: effect of pain levels, Pain, 45: 23-28.

Arntz, A., (1989). Cognitive aspects of the processing of repeated painful experiences., Paper presented at the First World Congress of Cognitive Therapy, Oxford.

Arntz, A., wan Eck. M. \& Heijmans, M., (1990). Predictions of dental pain: the fear of any expected ewil is worse than the evil itself, Behawiour Research and Therapy, 28: 29-41.

Arntz, A., Merckelbach, H., Peters, M. \& Schmidt, A. 11991). Chronic low back pain, response specificity and habituation to painful stimuli, Journal of Psychosomatic Research. 5 : $177-188$.

Arntz, An, Dreesen, L. \& Merckelbach, H. (1991). Attention, not anxiety influence pain. Behaviour Research and Therapy, 29:41.50.

Arntz, A. \& Lousberg. R., (1990). The effects of underestimated pain and their relationship to habituation, Behaviour Research and Therapy, 28: 15-28.

Arntz. A.\& Peters, M., (1990). Habituatie en pijnklachten, Gedragstherapie, 2: 90-109.

Arntz, A. \& wan den Hout, M., 1988). Generalizability of the matchimismatch model of fear, Behaviour Research and Therapy, 26: 207-223.

Atkinson, J., Kremer, E., Fisch, S., Morgam, C., Fryer Azad, R. E. C. \& Bloam, F., (1983). Plasma measures of beta-endorphin/beta-lipotropin-like immunoreactivity in chronic pain syndrome and psychiatric subjects, Psychiatry Research, 9:319-327.

Backman, E., Bengtsson, A., Bengtsson, M., Lennmarken, C. \& Henriksson, K., (1988). Skeletal muscle function in primary fibromyalgia. Effects of regional sympathetic blockade with guanethidine, Acta Neurologica Scandinavia, 77: 187-191.

Badia, P. Harley, J., (1970). Habituation and temporal conditioning as related to shock intensity and its judgement, Journal of Experimental Psychology, 84: 534-536.

Bakal, D. \& Kaganow, لJ, (1977). Muscle contraction and migraine headache: psychophysiological comparison, Headache, 15:208-216. 
Bakke, M., Tfelt Hansen, P., Oleson, J. \& Moller, E. (1982). Action of some pericranial muscles during prowoked atuacks of common migraine, Pain. 14:121-135.

Barsky, A. \& Wyshak, G., (1989). Hypochondriasis and related health attitudes, Psychosomatics, $30: 412-420$.

Beecher, H. (1959). The measurement of subjective responses, New York: Oxford Univ. Press.

Bengtsson, A. \& Henriksson, K., (1989). The muscle in fibromyalgia. A review of Swedish studies, Journal of Rheumatology, 16, suppl. 19: 144-149.

Besson, J. Chaouch, A., (1987). Peripherall and spinal mechanisms of nociception, Psychorogical Reviews, 67:67-186.

Bianchi, G. 11971). Origins of disease phobia, Austrelian and New Zealand Journal of Psychia. try, 5: 241-257.

Biedermann, $H_{2}$ (1984). Comments on the reliability of muscle activity comparisons in EMG biofeedback research with back pain patients, Biofeedback and Self-regulation, 9: $451-458$.

Biedermann, $H ., 11986$ ). Letters to the editor, Pain, 25: 411-412.

Biedermann, H., McGhie, A., Monga, T. \& Sharks, G., 11987). Perveived and actual control in EMG treatment of back pain, Behaviour Research and Therapy, 25: 137-147..

Bond, M., 11971). The relationship of pain to the Eysenck Personality Inventory, Cornelll Medical Index and Whitley Index of Hypochondriasis, Brithish joumal of psychiatry, 119: $671-678$.

Bourgoin, S., Le Bars, D. Clot, A. \& Hamon, M., (1988). Subcutaneous formalin induces a segmental release of Met-enkephaline-like material from the spinal cord, Pain, 9: 183-197.

Bourgoin, S., Le Bars, D., Clot, A., Hamon, M. \& Cesselin, F., (1990). Subcutaneous and evoked release of met-enkephalin-like material from the spinal cord, Pain, 41:323-329.

Brands, A. \& Schmidt, A., (1987). Learning processes in the persistence behavior of chronic low back pain patients with repeated acute pain stimulation, Pain, 30" 329-337.

Bromm, B. \& Scharein, E., (1982). Response plasticity of pain evoked reactions in man, Physiology \& Behavior, 28: 109-116.

Buchsbaum, M., Davis, G. \& Bunney, W., (1977). Naloxone alters pain perception and somatosensory evoked potentials in normal subjects, Nature, 270:620-622.

Bushnell, M., Duncan, G., Dubner, R. \& He, L., 11984). Activity of trigeminothalamic neurond in medullary dorsal horn of awake monkeys trained in a thermal discrimination task, Journal of Neurophysiology "52:170-187.

Cailliet, R., 11981). Low Back Pain Syndrome, Philadelphia: F.A. Davis Company.

Calsyn, D., Louks, J. \& Freeman, C., (1976). The use of the MMPI with chronic low back patients with a mixed diagnosis, Journal of Cinical Psychology, 32: 532-536.

Calvino, Bi. Villanueva, L. \& Le Bars, D., (1987). Dorsal horn (convergent) neurones in the intact anaesthetized arthritic rat. Il. Heterotopic inhibitory influences, Pain, 31: 359-379.

Calvino, B., Villaneuva, L. \& Le Bars, D., (1988). Heterotopic inhibitory influences on trigeminal rucleus caudalis convergent neurones in arthritic rats: evidence for the exacerbation of diffuse noxious inhibitory controls (DNIC), In: Besson, J.M. \& Guilbaud, G. (eds.), The Arthritic Rat as a Model of Clinical Pain?' Amsterdam: Elsevier: 101-109.

Calvino, B. \& Le Bars, D., (1986). The response to viscero-peritoneal nociceptive stimuli is reduced in experimental arthritic rats, Brain Research, 370: 191-195.

Cesselin, F., Bourgoin, S. Hamon, M., Artaud, F."Testut, M., Rascol, A. \& Montastruc, J.。 (1984). Normal CSF levels of met-enkephaline-like material in a case of naloxone-reversible congenital insensitivity to pain, Neuropeptides, $4: 217-225$.

Chapman, R., 1978). Pain: the perception of noxious events, In: Sternbach, R.A. led.l, The psychology of pain, New York: Raven Press: 169-202.

Chapmari, C. \& Bonica, J.. 11983). Acute Pain, Current Concepts, Kalamazoo: Upjohn Comp.

Chapman, C. \& Bonica, J. (1985). Chronic pain, Current Concepts, Kalamazoo: Upjohn Comp.

Chem, A., Treede, R. \& Bromm, B., (1984). Modulation of pain evoked cerebral potential by con-current subacute pain, in: Bromm, B. (ed.). Pain Measurement in Man, Amsterdam: Elsevier. 
Chen, A., Treede, R. \& Bromm, B., 1985). Tonic pain inhibits phasic pain: evoked cerebral potential correlates in man, Psychology Research, 14:343-351.

Chen, A. \& Treede, R. (1985). The MlcGill Pain Questionnaire in the assessment of phasic and tonic experimental pain: behavioral evaluation of the "pain inhibiting pain" effect, Pain, 22: $67 \times 79$.

Chitour, D., Dickenson, A. \& Le Bars, D., 11982). Pharmacological evidence for the involvement of serotonergic mechanisms in diffuse noxious inhibitary controls IDNIC). Brain Research, 236: $329-337$.

Cleeland, C. (1989). Measurement of pain by subjective report, In: Chapman, C.R. \& Loeser, J.D. (eds.), Advances in Pain Research and Therapy, New York: Raven Press: 391-404.

Cohen, M. Naliboff, B., Schandler, S. \& Heinrich, R., (1983). Signal detection and threshold measurement to loud tones and radiant heat in chromic low back pain patients and cohort controls, Pain, 6: 245-252.

Cohen, M., Swanson, G., Naliboff, B., Schandler, S. \& McArthur, D. (1986). Comparison of electromyographic response patterns during posture and stress tasks in chironic low back pain patients and controls, Journal of Psychosomatic Research, 30: 135.141.

Collier, J., Mehta, B. \& Spear, F., (1990). Experimental changes in pain threshold and severe pain threshold for electrically induced pain, Journal of Psychosomatic Research, 34: 99-102.

Collins, G., Cohen, M., Naliboff, B. \& Schandller, S., 11982). Comparative analysis of paraspinal and frontalis EMG, heart rate and skin conductance in chronic low back pain patients and normals to various postures and stress, Scandinavian Journal of Rehabilitation Medicine, Supplement: $39-46$.

Collpaert, F.. (1979). Can chronic pain be suppressed despite purported tolerance to narcotic analgesia?, Life Sciences, 24: 1201-1210.

Condes-Lara, M., Calvo, J. \& Fernandez-Guardioloa, A., (1981). Habituation to bearable experimental pain elicited by tooth pulp electrical stimulation, Pain, 11: 185-200.

Connally, G. \& Sanders, S., (1991). Predicting low back pain patients's response to lumbar sympathetic nerve blocks and interdisciplinary rehabilitation: the role of pretreatment overt pain behavior and cognitive coping strategies, Pain, 44:139-146.

Cox, G., Chapman, C. \& Black, R., (1978). The MMPI and chronic pain: the diagnosis of psychogenic pain, Journal of behavioural medicine, 1: 437-443.

Cram, J. \& Steger, J., (1983). EMG scanning in the diagnasis of chronic pain, Biofeedback and Self-regulation, 8: 229-241.

Dalton, J. \& Feuerstein, M., (1989). Fear, alexithymia and cancer pain, Pain, 38: 159-170.

De Bourreau, F., Luu, M. \& Doubrere, J., (1991). Study of experimental pain measures and nociceptive reflex in chronic pain patients and normal subjects, Pain, 44:131-138.

De Broucker, T., Cesaro, P., Willer, J. \& Le Bars, D. 11990 . Diffuse noxious inhibitory controls in man: involvement of the spino-reticular tract, Brain, 113:1223 1234.

Dehen, H., Willer, K., Prier, S., Boureau, F. \& Cambier, J., 11978). Congenital insensitivity to pain and the morphine like analgesic system, Pairi, 5:351 358 .

Dehen, H. Amsallem, B. \& Colas-Linthart, N. Cambier, J., 1986\%. Betaendorphine du liquide cephalo-rachidien au cours de l'insensibilite congenitale a al douleur, Review Neurologie, 142: $541 \cdot 544$.

Dellen, van H.J., Aasman, J. \& Mulder, J. M. G., 11985). Time domain versus frequency domain measures of heart rate viariability, In: Orlebeke, J.F., Mulder, G and van Doornen. C.J.P. leds.). Psychophysiology of Cardiovascular Control New York: Plenum Press.

Denko, C., Aponte, J., Gabriell, P. \& Petricevic, M. (1982). Serum beta-endorphin in rheumatic disorders, Journal of Rheumatology, 9: 827-833.

DeVries, H., (1968). EMG fatigue curves in postural muscles: apossible etiology for idiopathic back pain, American Journal of Physical Medicine, 47: 175-181.

Dickenson, A., Rivot, J., Chaouch, A., Besson, J. \& Le Bars, D., 1981). Diffuse noxious inhibitory controls (DNIC) in the rat with or without pCPA pretreatment, Brain Research. 2116: $313-321$. 
Dickenson, A., Le Bars, D. \& Besson, J.. 11981). Endogenous opiates and nociception: a possible functional role in both pain inhibition and detection as revealed by intrathecal naloxone, Neurascience Letters, 24: 161-164.

Dickenson, A., Chitour. D. \& Le Bars, D. 11983/. Influence of serotonergic receptor blockade on diffuse noxious inhibitory controls in the rat, In: Bonica, J.J., Advances in Pain Research and Therapy, Vol. 5, New York: Raven Press: 155-1160.

Dimitrijevic, M. Faganel, J. Gregoric, M. Nathan, P. \& Tronteli, J., (1972). Habituation effects of regular and stochastic stimulation. Journal of Neurology. Neurosurgery and Psychiatry. 35: $234-242$.

Dolce, J. \& Raczynskï, J., (1985). Neruramuscular activity and electromyography in painful backs: psychological and biomechanical models in assessment and treatment. Psychological Bulletin, 97: 502-520.

Ellertsen, B. \& Hammerborg, D., (1982). Psychophysiological response patterns in migraine patients, Cephalagia, 2:19-24.

El-Sobky, A., Dositrowsky, J. \& Wall, P., 11976). Lack of effect of nalloxone on pain perception in humains, Nature, 263: 783-784.

Epstein, S. \& Clarke, S., (1970). Heart rate and skin conductance during experimentally induced anxiety: effects of anticipated intensity of noxious stimulation and experience, Journal ar experimental Psychology, 84: 105-112.

Ernst, M., Lee, M. Dworkin, B. \& Zaretsky, H. (1986). Pain perception decrement produced through repeated stimulation, Pain, 26: 221-231.

Facchinetti, F., Martignoni, E., Gallai, V., Micieli, G., Petraglai, F., Nappi, G. \& Genazzani, A.. (1988). Neuroendocrine evaluation of central opioid activity in primary headache disorders. Pain, 34: 29-33.

Fewerstein, M., Bush, C. \& Corbisiero, R., 11982). Stress and chronic headache, Journal of Psychosamatic Research, 26: 167-182.

Feuerstein, M., Papciak, A. \& Hoon, P., 11987). Biobehavioral mechanisms of chronic low back pain, Clinical Psychology Review, 7: 243-273.

Fields, H., 11987/. Pain, New York: McGraw-Hill Book Company.

Flannery, J., 11977). Alexithymia, Psychotherapy and Psychosomatics, 28: 133-140.

Flor, H. \& Birbaumer, N., (1991). Comprehensive assessment and treatment of chronic back-pain patients without physical disabilities, In: Bond, M.R., Charlton, J.E., Woolf, C.J. (eds.). Proceedings of the V/th World Congress on Pain. Amsterdam: Elsevier: 229-234.

Flor, H., Birbaumer, N. \& Turk, D., (1985). Assessment of stress related psychophysiological reactions in chronic back pain patients, Journal of Consulting and Clinical Psychology, 53 : 354.364.

Flor, H., Birbaumer, N., Schugens, M. \& Lutzenberger, W., (1992). Symptom- specific psychophysiological responses in chronic pain patients, Psychophysiology, in press.

Flor, H., Birbaumer, N., Schulte, W. \& Roos, R., 11991). Stress-reliated ellectromyographic responses in patients with chronic temporomandibular pain, Pain, 46: 145-152.

Flor, H. \& Turk, D. 11988). Chronic back pain and rheumatoid arthritis:predicting pain and disability from cognitive variables, Journal of Behavioural Medicine, 11:251-256.

Flor, H. \& Turk, D. 11989). Psychophysiology of chronic pain: do chronic pain patients exhibit symptom-specific psychophysiological responses?, Psychological Butletin, 105: 215-259.

Fordvce, W., (1979). Environmental factors in the glenesis of low back paim, In: J.J. Bonica et al. leds.l. Advances in Pain Research and Therapy, Vol. 3. New York: Raven Press: $659-666$.

Fordyce, W. $11982 \%$. A behavioral perspective on chronic pain, British Journal of Clinical Psychology, 21: 313-320.

Fordyce, W., (1983). Behavioral conditioning concepts in chronic pain, In: J. Bonica (ed.). Advances in Pain Research and Therapy, Vol. 5.

Forgione, A. \& Barber, T., (1971). A strain gauge pain stimulator, Psychophysiology, 8: 102-106. 
Freeman, C., Calsy, D. \& Louks, J., 11976). The use of the Minnesota Multiphasic Personality Inventory with low back pain patients, Journal of Clinical Psychology, 32: 294-298.

Gannon, L., Haynes, S., Safranek, R. \& Hamilton, J., (1981). A psychophysiological investigation of muscle contraction and migraine headache, Journal of Psychosomatic Research. 25: $271-280$.

Gannon, L., Haynes, S., Cuevas, J. \& Chavez, R., (1987). Psychophysiological correlates of induced heladaches, Journat of Behavioral Medicine, 10:411.423.

Gannon, L. \& Haynes, S. (11986). Cognitive-physiological discordance as an etiological factor in psychophysiologicall disorders, Advances in Behavioural Research and Therapy, 8: 223-236.

Geers, A.. 11988). Kinesiologie: Onderzoek en Behandeling wan de Tonische en Fasische spieren, Lochem: De Tijdstroom.

Genazanni, A., Nappi, G., Facchinetti, F., Micieli, G. Petraglia, F., Bono, G., Monittola, C. \& Savoldi, F., 11984). Progressive impairment of CSF beta-EP levels in migraine sufferers, Pain, 18: 127-133.

Genazzani, A., Sandrini, G., Facchinetti, F., Rizzo, V. A. E. S. G., Calvani, M. \& Nappi, G., (1986). Effects of 1-5HTP with and without carbidopa on plasma beta-endorphin and pain perception: possible implications in migraine prophylaxis, Cephalalgia, 6: 175-179.

Gerhart, K., Yezierski, R., Giesler, G. \& Willis, $W_{n .}$ (1981). Inhibitory receptive fields of primate spinothalamic tract cells, Journal of Neurophysiology, 46: $1309-1325$.

Glaser, E., Hall, M. \& Whittow, G., (1959). Habituation of the heating and the cooling of the same hand, Journat of Physiology, 146: 152-164.

Goldstein, 1., (1970). Electromyography, a measure of skeletal muscle response, In: N.S Greenfield \& R.A. Sternbach. Handboak of psychophysiology, New York: Holt, Rhinehart and Winston: $329-349$.

Goldstein, A.. (1979). Endorphins and pain: a critical review, In: Beers, R.F. \& Bassett, E.G., Mechanisms of pain and analgesic campounds, New York: Raven Press: 249-262.

Goodman, A. (ed.), (1985). The pharmacological basis of therapeutics (7th ed), Now York: McMillan Publishing Company.

Gracely. R., (1979). Psychophysical assessment of human pain, In: Bonica, J.J., Advances in Pain Research and Therapy, 3, New York: Raven Press: 805.824.

Gracely, R., (1984). Subjective quantification of pain perception, In: Bromm, B. (ed.), Pain Measurement in Man. Neurophysiological Correlates of Pain, Amsterdam: Elsevier: $371-387$.

Graham, F., (1979). Distinguishing among orienting, defense and startle reflexes, In: Kimmel, H.D., van Olst, E.H. \& Orlebeke, J.F. leds.). The Orienting Reflex in Humans, Hillsdale: Erlbaum.

Gray, J., (1975). Elements of a two-process theory of learning, London: Academic Press.

Grevert, P. \& Goldstein. A., (1978). Endorphins: naloxone fails to alter experimental pain or mood in humans, Science, 199: 1093-1095.

Groves, P. \& Thompson, R., [1970). Habituation: a dual-process theory., Psychological Review, 77: $419-450$.

Hamon, M. Bourgoin, S., Le Bars, D. Cesselin. F., 11988\%, In vivo and in vitro release of central neirotransmitters in relation to pain, In: Fields, H.L. \& Besson, J.M. (eds .), Progress in Brain Research Vol 77: Pain Modwlation, Amsterdam: Elsevier: 431-444.

Hardebo, J., Ekman, M., Eriksson. M., Holgersson, S. R Rybers, B., 119851. CSF opioid levels in cluster headache, In: Rose, C. led. h, Migraine, Basel: Karger.

Haslam, D., 1966). The effect of threatened shock upon pain threshold, Psychosomatic Science, 6, 309-310.

Haynes, S., Gannon, L., Cuevas, J.. Heiser, P., Ha, ilton, J. Katrandides, M., 119831. Assessment of muscle contractions in headache and non-headache conditions, Psychophysiology, 20: $393-399$.

Hazouri, L. Mueller, A., (1950). Pain threshold studies on paraplegic patients, Archives of Neurology and Psychiatry, 5: 607-613. 
Helson, H., 119641. Adaptation-Level Theory, an expermental and systematic approach to behawior, New York: Harper \& Row.

Hendler. N., 119811. Diagnosis and monsurgical management of chronic pain. New Vork: Raven Press.

Hodgsion, R. Bachman; S., 11974 ). Desynchrony of measure of fear, Behaviour Research and Therap. 12: 319-326.

Holmes, T, Wolf, H., 11952). Life situation, emotions and backache, Psychosomatic Medicine, Ha: $18-33$.

Hotroyd, K., Pentzien, D. Hursey, K., Tobin, D., Rogers, L., Holm, J., Mancille, P., Hall, ل. \& Chila, A., 119841. Change mechanisms in EMG biofeedback training: cognitive changes underlying improvements in tension headlache, Journal of Consulting and Clinical Psycholo$g V^{2}, 52: 31-50$

Hoyt, W., Hunt, H, de Pauw, M., Bard, D. S.F., Passias, J., Robbins, D., Runyon, D., Semrad, S., Symonds, J. \& Watt, K., (1981). Electromyographic assessment of low-back pain syndrome, Journal of the American Osteopathic Association, 80: 722-730.

Hu, J., (1990). Response properties of nociceptive and non-nociceptive neurons in the rat's trigeminal subnucleus caudalis (medullary dorsal horn) related to cutaneous and deep craniofacial afferent stimulation and modulation by diffuse noxious inhibitory controlls, Pain, $41: 331-343$.

Hugon, M.. (1973). Exteroceptive reflexes to stimulation of the sural nerve in normal man, In: Desmedt, 1. E. (ed.), New Developments in Electromyography and Clinical Neurophysiology, Basel: Karger.

Hursey, K., Holroyd K., Penzien, D. Holm, J. (1985). The influence of pain state on physiological reactivity of tension headache sufferers, Headache, 25: 79-84.

Huskisson, E. \& Hart, F. (1972). Pain threshold and arthritis, British Medical Journal, 28 October: $193-195$.

lacono, W. \& Lylkken, D.* (1984). The effects of instructions and an engaging visual task on habituation to loud tones: An evaluation of an alternative to the traditional habituation paradigm, Physiological Psychology, 12:23-29.

ladorala, M., Brady, L., Draisci, G. \& Dubner, R., 11988\%. Enhancement of dynorphin gene expression in spinal cond following experimental inflammation: stimulus specificity, behavioral parameters and opioid receptor binding, Pain, 35: 313-326.

International Association foir the Study of Pain, 119791. Pain terms: a list with definitions and notes on usage, Pain, 6: $249-252$.

Jackson, J., (1974). Amplitude and habituation of the orienting reflex as a function of stimulus intensity, Psychophysiology, 11: 647-659.

James, F. \& Large, R., (1991). Quantifying alexithymia and hypnotisability in pain clinic attenders and nomal controls, in: Bond, M.F., Chariton, J.E. Wolf, C.J. leds.l, Proceedings of the Whth world congres on pain. Amsterdam: Elsever Science publishers: $247-250$.

Janda, $V,(1978)$. Muscles, central nervous motor regullation and back problems, In: 1 . Korr (od.). The Neurobiologic Machanisms in manipulative therapy, New York: Plenum: 27-41.

Jayasinghe, W., Harding, R., Anderson, J. \& Sweetman, B., (EMG, CLBP). An electromyographic inwesigation of postural fatigue in chronic low back pain, Neurophysiology, 1978: $191-498$.

Jurna, 1. (1988). Dose-dependient inhibition by naloxone of nociceptive activity evoked in the rat thialamus, Pair, $35: 349-354$.

Kayser. V.,Besson, J. \& Guilbaud, G., (1988). Paradoxical effects of low doses of naloxone in experimental models of inflammatory pain. ln: Fiells, H.L. \& Besson, J.M., Progress in Brain Research, Vol. 22, Amsterdam: Elsevier: 301-312.

Kirkaldy-Willis, W., 11988). The pathology and pathogenesis of low back pain, In: Kirkaldy-Willis, W.H., Managing low back pain (2ed), New York: Churchill Livingstone: 49-55.

Korbon, G., DeGood, D., Schroeder, M., Schwartz, D. \& Shutty, M. (1987). The dlevelopment of a somatic amplification rating scale for low back pain, Spine, 12:787-791. 
Kraus, E., Besson, J. \& Le Bars, D., (1982). Behavioral model for diffuse noxious inhibitory controls (DNIC): potentiation by 5-hydroxytryptophan, Brain Research, 231: 461-465.

Kravitz, E., Moore, M. \& Glaros, A., 11982). Parallumbar muscle activity in chronic low back pain, Archives of Physical and Medical Rehabilitation, 62:172-176.

Kroner, B., (1984). Psychophysialogische Korrelate chronischer Kopfschmerzen, Zeitschrift fur experimentelle und angewandte Psychalogy. 31: 610-639.

Kroner-Herwig, B., Diergarten, D., Diergarten, D. \& Seeger-Siewert, R., 1988). Psychophysiological reactivity of migraine sufferers in conditions of stress and rellaxation, Jounnal of Psychosamatic Research, 32: 483-492.

Lang, P. (1968). Fear reduction and fear behaviour: problems in treating a construct, Research in Psychotherapy, 3: 90-102.

Lang, $P_{.},(1971)$. The application of psychophysiological methods to the study of psychotherapy and behavior modification, In: Bergin \& Garfield (eds.). Handbook of Pswchotherapy, New York: Wiley.

Langemark, M., (1989). Naloxone in moderate dose does not aggravate chronic tension headache, Pain, 39: 85-93.

Le Bars, D., Dickenson, A. \& Besson, J., (1979). Diffuse noxious inhibitory controls (DNIC) I. Effects on dorsal horn convergent neurons in the rat, Pain, 6: 283-304.

Le Bars, D., Dickenson, A. \& Besson, J. (1979b). Diffuse noxious inhibitory controls (DNIC). H. Lack of effect on non-convergent neurones, supraspinal involvement and theoretical implications, Pain, 6: 305-327.

Le Bars, D., Chitour, E., Kraus, E., Dickenson, A. \& Besson, J., 19981). Effects of naloxone upon DNIC in rat, Brain Research, 204: 387-403.

Le Bars, 1982, Roby, A. \& Willer, J., (1982). Effects of heterotopic thermal stimuli on a nociceptive reflex and the related pain sensation in man, Physiological Sociery. Nov., $31 \mathrm{p}$.

Le Bars, D. Dickenson, A. \& Besson, J., (1982b). The triggering of bulbo-spinal serotonergic inhibitory controls by noxious peripheral input, In: Sjolund, B. \& Bjorklund, A. (eds.), Brain Stem Control of Spinal Mechanisms, Amsterdam: Elsevier.

Le Bars, D., Dickenson, A., Besson, J. \& Villanueva, L.. 11986). Aspects of sensory processing through convergent neurons, In: Yaksh, T.L., Spinal Afferent Processing, New York: Plenum Press: 467-49.

Le Bars, D., Willer, J., De Broucker, T. \& Villanueva, L., (1989). Neurophysiological mechanisms involved in the pain relieving effects of counterirritation and related techniques including acupunture, In: Stux, G. (Ed.), Scientific Basis of Acupuncture, Berlin: Springer Verlag: 79-112.

Le Bars, D. \& Villanueva, L., (1988). Electrophysiological evidence for the activation of descending inhibitory controls by nocicpetive afferent pathways, In: Fields, H.L. \& Besson, J.M. Pain Modulation, Amsterdam: Elsever: 275-300.

Le Bars, D. \& Willer, J., (1988). Letter to the Editor, Pain, 32: 259-260.

Le Blanc, d. \& Potvin, P., (1966). Studies on habituation to cold pain, Canadian Journal of Physiology and Pharmacology 44: 287-239.

Lethem, J., Slade, P., Troup, J. \& Bentlev, G., (1983). Outline of fear-avoidance model of exaggerated pain perception-1, Behaviour Research and Therapy, 4:401-408.

Levine, J., Gordon, N., Jones, R. \& Fields, H. 11978). The narcotic antagonist naloxone enhances clinical pain, Nature, 27: 826-827.

Levine, J., Gordon, N. \& Fields, H., 11979). Naloxone dose dependently produces analgesia and hyperalgesia in postoperative pain, Nature, 278: 740-741.

Lewis, T. \& Keligren, J., (1939). Observations relating to referred pain, viscero-motor reflexes and other associated phenomena, Clinical Science, 4: 47-71.

Lindblom, U. \& Tegner, R., (1979). Are the endorphins active in clinical pain states? Narcotic antagonism in chronic pain patients, Pain. 7: 65-68.

Lipman, J., Blumenkof, B. \& Parris, W., (1987). Chronic pain assessement using heat beam dolorimetry, Pain, 30: 59-67. 
Lloyd, M. \& Appel, J. 11976). Signal detection theory and the psychophysics of pain: An introduction and rewiew, Psychosomatic Medicine, 38:79-94.

Lynn, B. \& Pen, E. (1977). A comparison of four lests for assessing the pain sensitivity of different subjects and test areas, Pain, 3: 353-365.

Matow, R., Grimm, $L$. Olson, $R_{\text {. }}$ (1980). Differences in pain perception between myofasicial pain dysfunction patients and normal subjects: a signal detection analysis, Jounal of Psychosomatic Research, 24: 303-309.

Malow, A. \& Olson, R., (1981). Changes in pain perception after treatment for chronic pain, Pain, 11:65-72.

Martin, J. \& Phil, R., (1985). The stress-alexithymia hypothesis: theoretical and empirical considerations, Psychotherapy and Psychosomatics, 43: 169-176.

Martin, J. \& Phil, A., (1986). Influences of alexithymic characteristics on physiological and sulb-

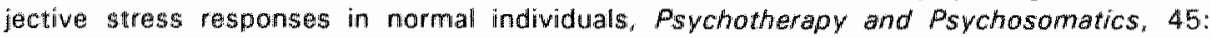
$66-77$.

Mayer, D., 11979/. Endogenous anaigesia systems: neural and behavioral mechanisms, In: Bonica, J.J. Advances in Pain Research and Therapy, Vol 3. New York: Raven Press: $385-410$.

McNemar, G., (1969). Psychological statistics, Chapter 10. Factars which affect the correlation coefficient, New York: Willey \& Sons: 154-163.

Melzack, R. \& Loeser, J., (1978). Phantom body pain in paraplegics: evidence for a central "pain generating mechanism", Pain, 4: 195-210.

Melzack, R. \& Wall, P., (1965). Pain mechanisms: A new theory, Science, 150: 971-979.

Mendelsohn, $G$.. 11982). Alexithymia and chronic pairi: prevalence, correlates and treatment results, Psychotherapy and Psychosamatics, 37: 154-164.

Menetrey, D.. Lambard, M. \& Besson, J., (1988). Electrophysiological properties of dorsal ham nociceptive newrons in the rat and the response to the intravenous administration of naloxone, In: Besson, J.M. \& Guilbaud, G. The arthritic rat as a model of clinical pain?, Amsterdam: Elsevier Science Publishers: 67-81.

Merskey, H. \& Evans, P., 11975). Variations in pain complaint threshold in psychiatric and neurological patients with pain, Pain, 1: 73-79.

Millan, M., (1986). Multiple opiaid system and pain, Pain, 27: 303-346.

Millan, M., Millan, M., Czlonkowski, A., Hollt, V. \& Pilcher, C. H. A. \& Colpaert, F.C., 119861. A model of chronic pain in the rat: response of multiple opioid systems to adjuvent-induced arthiritis, The Journal of Neuroscience, 6: 899-906

Millan, M., Czlonkowski, A , Pilcher, C., Almeida, O., Millan, M., Colpaert, M. \& Herz, A., 119871. A model of chronic pain in the rat: functional correlates of alterations in the activity of opioid systems, The Journal of Neuroscience, 7:77.87.

Millan, M., Czlonkowski, A., Morris, B. S. C., Arendt, R., Huber, A., Hollt, V. \& Herz, A., (1988). Inflammation of the hind limb as a model of unilateral, localized pain influence on multiple opioid systems in the spinal cord, Pain, 35:299-312.

Molin. C., Edman, G. \& Schalling, D., (1973). Psychological studies of patients with mandibular dysfunction syndrome, Swedish Dental Journal, 66; 15-23.

Morley, S. (1985). An experimental investigation of some assumptions underpinning psychological treatments of migraine, Behavioural Research and Therapy, 23: 65-74.

Morton, C., Du, H. Xiao, H., Maisch, B. \& Zimmerman, M., 11988). Inhibition of nociceptive responses of lumbar dorsal horn neurones by remote noxious afferent stimulation in the cat, Paim, 34: 75-83.

Mumford. J., 11965\%. Pain perception threshold and adaptation of normal human teeth, Archives of Oral Biology. 10: 957-968.

Myrtek, M. Spital, S., (1986). Psychophysiological response patterns to single, double and triple stressors, Psychophysiology, 23: 663-671.

Naliboff, B. \& Cohen, M., (1989). Psychophysical laboratory methods applied to clinical pain patients, In: Chapman, C.R. \& Loeser, J.D., Advances in Pain Reserach and Therapy, Vol. 12. New York: Raven Press: $365-389$. 
Naliboff, B., Cohen, M., Schandler, S. Heinrich, R., 19811. Signal detection and threshold measurement for chronic back pain patients, chronic illness patients and cohort controls to radiant heat stimuli, Journal of Abnormal Psychology, 90: 271-274.

Naliboff, B., Cohen, M., Swanson, G., Bonebakker, A. \& McArthur D.L... (11985). Comprehensive assessment of chronic low back pain patients and controls: physical abilities, level of activity, psychological adjustment and pain perception, Pain, 23: 121-134.

Nappi, G., Facchinetti, F., Martignoni, E., Petraglia, F., Bono, G., Micieli, G. R. C., Manzoni، G. \& Genazzani, A, (1985). Plasma and CSF endorphin levels in primary and symptomatic headaches, Headache, 25: 1411-144.

Nouwen, A. \& Bush, C. (1984). The relationship between paraspinal EMG and chronic low back pain, Pain, 20: 109*123.

Nouwen, A., Akkerveeken, P. \& Versloot, J., 11987). Patterns of muscular activity during movement in patients with chronic low-back pain, Spine, 12:777-782.

O'Brian, R. \& Kaiser, M., (1985). MANOVA method for analyzing repeated measures designs: an extensive primer, Psychological Bulletin, 97: 316-333.

Ohman, A., (1979). The orienting response, attention and learning: An information processing perspective, In: Kimmel, H.D., van Olst, E.H. \& van Olst, E.H. (eds.), The orienting reffex in humans, Hillsdale: Erlbaum: 443-471.

wan Olst, E., Kak, A. \& Orlebeke, J., (1980). In/eiding in de Psychophysiologie. Deventer: Van Loghum Slaterus.Packer, J. \& Siddle, D., (1987). The effects of signal value on short-and long-term habituation, Biological Psychology, 24: 261-273.

Pantaleo, T., Duranti, R. \& Bellini, F., (1988). Effects of vibratory stimulation on muscular pain threshold and blink response in human subjects, Pain, 24:239-250.

Papciak, A., Feuerstein, M. \& Spiegel, J. (1985). Stress reactivity in alexithymia: decoupling of physiological and cognitive responses, Journal of Human Stress, 11: 135-142.

Passchier, J.. (1987). Migraine, spanningshoofdpijn en stress, Pijninformatorium, Alphen a/d Rijn: Samson: HP 1500.

Passchier, J. \& Orlebeke, J., (1983). Slow habituation in migraine patients: do they invest to much attentional capacity?, Headache, 23: 198-199.

Pederson, H., Blench, C. \& Gardner, E., (1956). The anatomy of lumosacral posterior rami and meningeal branches of spinal nerves (sinu-vertebral nerves), Journal of Bone and Joint Surgery, 38: 377-391.

Pennebaker, J., (1982). The Psychology of Physical Symptoms, New York: Springer Verlag.

Pennebaker, $J$. \& Lighner, $J$, (1980). Competition of internal and external information in an exercise setting, Journal of Personality and Social Psychologv "39: 165-174.

Perry. F., Heller, P. \& Levine, J., 1988). Differing correlations between pain measures in syndromes with or without explicable organic pathology, Pain, 34: 185-189.

Perry, F., Heller, P. \& Levine, J., (1991). A possible indicator of functional pain: poor pain scale correlation, Pain, 46: 191-193.

Pertovaara, A., Kemppainen, P., Johansson, G. Karonen, S.. (1982\%. Ischemic pain nonsegmentally produces a predominant reduction of pain and thermal sensitivity in man: a selective role for endogenous opioids, Brain Research, 251:83-92.

Peters, M., Schmidt, A. \& van den Hout, M. (1989). Chronic low back pain and the reaction to repeated acute pain stimuli, Pain, 39: 69-76.

Peters, M., van den Hout, M. \& Schmidt, A. Supression of the nociceptive flexion reflex by two different counter-irritation stimuli, Submitted for publication.

Peters, M. \& Schmidt, A., (1992). Differences in pain perception and sensory discrimination between chronic low back pain patients and healthy controls, Journal of Psychosomatic Research, 36: 47-53.

Philips, H., (1987). Avoidance behavior and its role in sustaining chronic pain, Behaviour Research and Therapy, 25: 273-279.

Philips, H. \& Hunter. M.. (1982). A psychophysiological investigation of tension headache, Headache, 22: 173-179. 
Philips, H. \& Jahanshahi, M. 119851. Chronic pain: an experimental analysis of the effects of exposure, Behaviou Research and Therapy, 23: 281-290.

Pilowski, I. Spence, N., (1975). Patterms of illness behaviour in patients with intractable pain. Pain, 19: 279-202.

Plaghki, L., Delisle, D. Godfraind, J., (1990). Pain perception induced with short CO2-laser stimuli is modulated by heterotopic nociceptive conditioning stimulation. TENS and mental task: a sensory decision theory analysis, Poster at the ENA congress, Stockholm.

Postone, N., (1986). Alexithymia in chronic pain patients, General Hospital Psychiatry, 8: $163-167$.

Price, D. \& Mctlaffie, J. 11988). Effects of heterotopic conditioning stimuli on first and second pain: a psychophysical evaluation in humains, Pain, 34: 245-252.

Pritchard, D. \& Wood, M. (1983). EMG levels in the occipitofrontalis muscles under an experimental stress condition, Biofeedback and Self-regulation, 8: 165-175.

Procacci, P., (1979). Methods for the study of pain threshold in man, In: Bonica, J.J., Advances in Pain Research and Therapy, Amsterdam: Raven Press: 781-790.

Procacci, P., Zoppi, M. \& Maresca, M., 11979). Experimental pain in man, Pain, 6: 123-140.

Przewlocka, B., Lason, W. \& Pzrewlocki, Ri., (1986). The release of opioid peptides from the rat spinal cord, In: Tucek, S. et al. leds.), Molecular Basis of Neural Function, Euopean society of neurochemistry: 243 .

Rabavilas, A., Stefanis, C.. Liappas, J., Perissaki, C. \& Rinieris, P., (1982). Synchrony of subjective and psychophysiological responses in involutional depression, Neuropsychobiology, 8: 156-161.

Rachman, S. \& Lopatka, C.. (1986). Match and mismatch in Gray's theory-ll Behaviour Research and Therapy, 24:395-401.

Riley, J. \& Levine. F.M.,. (1988). Courterstimulation and pain perception: effects of electrocutaneous vs, auditory stimulation upon cold pressor pain, Pain, $35: 259-264$.

Robinson, J., Whitsett, S. \&aplan, B., (1987). The stability of physiological reactivity over multiple sessions, Brological Psychology, 24: 1291139.

Roby-Brami, A., Bussel, B., Willer, J. \& Le Bars, D., (1987). An electrophysiological investigation into the pain-felieving effects of heterotopic nociceptive stimuli. Probable involvement of a supraspinal lloop, Brain, 110: 1497-1508.

Fojahn, J \& Gerhards, F., 11986). Subjective stress sensitivity and physiological responses to an aversive auditory stimulus in migraine and control subjects, Journal of Behavioral Medicine, 9: 203-212.

Rollman, G., (1979). Signal detection theory pain measures: empirical validation and adaptation-levell effects, Pain, 6: $9-21$.

Rollman, G., (1979). Adaptation Level Effects in the rating of acute pain, In: Bornica, Jl.J., Advances in Pain Research and Therapy, Vol. 3, New York: Raven Press: 825-829.

Rollman, G., (1983). Measurement of experimental pain in chronic paim patients: methodological and individual factors, In: Melzack, R. (ed.), Pain Measurement and Assessment. New York: Raven Press: 251-257.

Rosomoff, H. Fishbain, D., Golldberg, M., Santana, R. \& Rosomoff, R., 11989). Physical findings in patients with chronic intractable benign pain of the neck and/or back, Pain. 37 : $279-287$.

Roy, S. Carlo, M. De Luca, J. \& Casavant, D. (1988). Lumbar muscle fatigue and chronic lower back pain, Spine, 14: 992-1001.

Sandrini, G., Alfonsi, E., Sances, G. F. F., Bono, G. \& Nappi, G., (1985). Nociceptive flexion reflex threstiold in migraine sufferers, Cephalagia, 5:26-27.

Scheier, M, Carver, C. \& Matthews, K, (1983). Attentional factors in the perception of bodily states, In: Cacioppo, J.T. \& Petty, R.E., Social Psychophysiology, New York: Guilford Press: $510-542$.

Schmidt, A., (1985). Performance level of chronic low back pain patients in different treadmill test conditions, Journal of Psychosomatic Research, 6: 639-645. 
Schmidt, A., (1986). Persistence behavior of chronic low back pain patients - a medical psychological study, Maastricht: Academic Dissertation.

Schmidt, A. \& Brands, A. (1986). Persistence behavior of chronic low back patients in an acute pain situation, Journal of Psychosomatic Research, 30:339-346.

Schmidt, A., Gierlings, R. \& Peters, M., 11989). Enwironmental and interoceptive influences on chronic low back pain behavior, Pain, 38: 137-143

Schouenborg, J. \& Dickenson, $A_{\text {. }}$, (1985). The effects of distant noxious stimulation an $\mathrm{A}$ and $C$ fiber-evoked flexion reflexes and neuronal activity in the dorsal horm of the rat, Brain Research, 328: 23-32.

Scudds, R., Rollman, G., Harth, M. \& McCain, G., 11987). Pair perception and personality measures as discriminators in thr classification of fibrositis, Journal of Rheumatology, 14: 563-569.

Seltzer, S. \& Seltzer, J. (1986). Tactual sensitivity of chronic pain patients to non-painful stimuli, Pain, 27: 291-295.

Siddle, D. \& Heron, P., 11976). Reliability of electrodermal habituation measures under two conditions of stimulus intensity, Journal of Experimental Research in Personality, 5 : 295. 303.

Siegel, S., (1956). Nonparametric Statistics far the Behavioral Sciences, New York.: McGraw-Hill.

Skjelbred \& Lokken, (1983). Effects of naloxone on post-operative pain and steroidlinduced analgesia, British Journal of Clinical Pharmacologw, 15: 221-226.

Slade, P., Troup, J., Lethem, J. \& Bentley, G., (1983). The fear-avoidance madel of exaggerated pain perception - 11, preliminary studies of coping strategies for pain. Behaviour Research and Therapy, 21:409-416.

Soderberg, G. \& Barr, J., (1983). Muscular function in chronic low back dysfunction, Spine, 8: 88-93.

Sokolov, E., (1960). Neuronal models and the orienting reflex, In: Brazier, M. led.), The Centrat Nervous System and Behaviour, Boston. Josiah Macy Jr. Foundation.

Sokolov, E., (1963). Perception and the Conditioned Reflex, Oxford: Pergamon Press.

Spanos, N., Radtke-Bodorik, H., Ferguson, J. \& Jones, B., (1979). The effect of hypnotic suspectibility, suggestions for analgesia, and the utilization of cognitive strategies on the reduction of pain, Journal of A.bmormal Psychology, 8.8: 282-292.

Stacher, G. Abatzi, T., Schulte, F., Schneider, C., Stacher-Janotta, G., Gaupman, G., Mittelbach, G. \& Steinringer, H., (1988). Naloxone does not alter the perception of pain induced by electrical and thermal stimulation of the skin in healthy humans, Pain, 34 : $271-276$.

Sternbach, R.x (1968). Pain: A psychophysiological analysis, physiological responses, New York: Aciademic Press, Chapter 4.

Stembach, $\mathbb{R} .,(1974)$. Pain Patients: Traits and Treatment, New York: Academic Press.

Stembach, R., (1978). Clinical aspects of pain. In: Sternbach, R.A. Ied.1. The Psychology of Pain, New York: Rawen Press.

Stembach, R. Wolf, S., Murphy, R. \& Akeson. W., 11973). Traits of pain patients: the low-back "loser", Psychosomatics, 14: 226-229.

Stermbach, R. \& Tursky, B. 11965\%. Ethric differences among housewives in psychophysical and skin potential responses to electric shock, Psychophysiology, $1: 241-246$.

Strempel, H., (1976). Adaptive Modifikationen des Kalteschmerzes, Europian Journal af Applied Physiology, 36: 19-25.

Sturgis, E., Schaefer, C., Ahles, T. \& Thomas, L., 119841. Effect of movement and prosition in the evaluation of tension headache and nonheadache control subjects, Headache, 24 : 88-93

Talbot, J., Duncan, G., Bushnell, M. \& Boyer, M., 1987). Diffuse noxious inhibitary contrals (DNICs): psychophysical evidence in man for intersegmental suppresion of moxious heat perception by cold pressor pain, Pain, 30: 221-232. 
Tabot, J., Duncan, G. \& Bushnelf, M., (1989). Effects of diffuse noxious irihibitory controlss (DNiCs) on the sensory discriminative dimension of pain perception, Pain, 36: 231-238.

Terenius, L., 11979). Endorphins in chronic pain, In: Bonica, J.J., Advances in Pain Research and Therapy, Vol. 3, New York: Raven Press: 459-471.

Terenius, $1 ., 11982 \%$. Neuropeptides in human cerobrospinal fluid - relation to pain. In: Sjalund, B. \& Bjorklund, A. (eds.), Brain Stem Control of Spinal Mechanisms, Amsterdam: Elsevier: 509-518.

Terenius, L., (1987). Endorphins and substance $P$ in chronic pain, In: Tiengo, $M$. (ed.), Advances in Pain Research and Therapy, Vol. 10, New York: Raven Press: 9-15.

Terenius, $\mathrm{L}$., 11988). Significance of opioid pepetides and other potential markers of neuropeptide systems in cerobrospinal fluid, ln: Fields, H.L. \& Besson, J.M. (eds.), Pain Modulation. Amsterdam: Elsevier: 419-430.

Terman, G., Lewis, J. \& Liebeskind, J., (1984). Endogenous pain inhibitory substrates and mechanisms, In: $C$. Benedetti et al., Advances in Pain Riesearch and Therapy, Vol. 7. New York: Raven Press: 43-56.

Thompson, J. Adams, H., (1984). Psychophysiological characteristics of headache patients, Pain, 18:41-52.

Thompson, R. \& Spencer, W., (1966). Habituation: A model phenomenon for the study of neuronal substrates of behavior, Psychological Review, 73: 16-43.

Tomlinson, R., Gray, B. \& Dostrovsky, J., (1983). Inhibition of rat spinal cord dorsal horn neurons by non-segmental, noxious cutaneous stimuli, Brain Research, 279: 291-294.

Tricklebank, M. \& Curzon, G., 1984\}. Stress-induced Analgesia, John Wiley \& Sons Ltd.

Turk, D. \& Flor, H., (1984). Etiological theories and tireatment for chronic back pain II. Psychological models and interventions, Pain, 19: 209-233.

Turpin, G., (1986). Effects of stimulus intensity on autonomic responding: the problem of differentiating orienting and defense reflexes, Psychophysiology, 23: 1-14.

Turpin, G. \& Siddle, D., (1978). Cardiac and forearm plethysmographic responses to high intensity auditory stimulation, Biological Psychology, 6: 267-281.

Tursky, B., (1973). Physical, physiological, and psychological factors that affect pain reaction to ellectric shock, Psychophysiology, 11:95-112.

Tyrer, P. Lee, I. \& Alexander, J., (1980). Awareness of cardiac function in anxious, phobic and hypochondriacal patients, Psychological Medicine, 10:171-174.

Ueda, H., Fukushima, N., |Kitao, T., Ge, M. \& Takagi, H., (1986). Low doses of naloxone produce analgesia in the mouse brain by blocking presynaptic autoinhibition of enkephalin rellease, Neuroscience Letters, 65: 247-252.

Vallfors, B., \{1985\}. Acute, Subacute and Chronic Low Back Pain, Scandinavian Journal of Rehabilitation Medicine, Suppl. 11: 1-98.

Villanuewa, L.. Cadden, $S$. \& Le Bars, D. (1984). Evidence that diffuse noxious inhibitory controls $\mid D N \| C]$ are mediated by a final post-synaptic inhibitory mechanism, Brain Rew search, 298: 67-74.

Villanueva, L. Cadden, S. \& Le Bars, D., (1984b). Diffuse noxious inhibitory controls (DNIC): evidence for postwsynaptic inhibition of trigeminal nucelus caudalis convergent neurones, Brain Research, 321: 165-168.

Villanuewa, ${ }_{\text {. }}$ Peschanski, M., Calvino, B. \& Le Bars, D. (1986). Ascending pathwayws in the spinal cord involwed in triggering of diffuse noxious inhibitory controls in the rat, Journal of Neurophysiology, 55: 34-55.

Vlaeven, al. 11991). Chronic low back pain, assessment and treatment from a behaviorat rehabilitation perspective, Amsterdam: Swets \& Zeitlinger.

Waddell, G. McCulloch, J., Kummel, E. \& Verner, R. (1980). Non-organic physical signs in low bacik pain, Spine, $5: 117-125$.

Wagner, A.. (1976). Priming in STM: An infomation-processing mechanism for self-generated or retrieval-generated depression in performance, In: Tighe, T. \& Leaton, R. (eds.), Habituation: perspectives from child development, animal behavior, and neurophysiology. Hillsdale: Eplbaum: 95-128. 
Wagner, A, (1979). Habituation and memory, In: Dickenson, A. \& Boakes, R., Mecharisms of learning and motivation: A memorial volume to Jerzy konorksì Hillsdale: Eribaum: 53-82.

Wagner, A. 119811. SOP: A modiel of automatic memory processing in naimal behavior, in: Spear, N.E. \& Miller, R.R., Information processing in animals: Memory mechanisms. Hillsdale: Erlbaum: $5-48$.

Wand-Tetley, J. 11956$)$. Historical methods of counter-irritation, Annals of Physical Medicine. 3: $90-98$.

Watkins, L. (1989). Algesiometry in laboratory animals and man:current concepts and future directions, in: Chapman, C.R. \& Loeser, J.D. leds.l. Advances in Pain Research and Therapy, New York: Raven Press: 249-265.

Watson, D. (1982). Neurotic tendencies among chronic pain patients: an MMPI item analysis, Pain, 14: 365-385.

Wilfling, F., (1981). Psychophysiological correlates of low back pain, Dissertation Abstracts International, 42: 2592-B.

Willer, J., (1977). Comparative study of perceived pain and nociceptive flexion reflex in man, Pain, 3: 69-80.

Willer, J., Dehen, H., Boureau, F. \& Cambier, J., (1978). congenital insensitivity to pairn and nalloxone, The Lancet, September 30: 739.

Willer, J., Roby, A. \& Le Bars, D. (1984). Psychophysical and electrophysiological approaches to the pain relieving effects of heterotopic nociceptive, Brain, 107: 1095-1112.

Willer, J., Bergeret, S. \& Gaudy, J., 11985\%. Epidural morphine strongly depresses nociceptive flexion reflexes in patients with postoperative pain, Anesthesiology, 63: 675-680.

Willer, J., Barranquero, A., Kahn, M. Salliere, D. (1987). Pain in sciatica depresses lower limb nociceptive reflexes to sural nerve stimulation, Journal of Neurology, Neurosurgery, and Psychiatry, 50: $1-5$.

Willer, J., De Broucker, T. \& Le Bars, D., (1989). Encoding of nociceptive thermal stimuli by diffuse noxious inhibitory controls in humans, Journal of Neurophysiologw, 62: 1028-1038.

Willer, J., Le Bars, D. \& De Broucker, T., (1990). Diffuse noxious intibitory controls in man: involvement of an opiodergic link. European Journal of Pharmacology. 182: 347-355.

Williams, J. \& Spitzer, R., (1982). Idiopathic pain disorder: a critique of pain prone disorder and a proposal for a revision of the DSM-Ill category psychogenic pain disorder, Jaurnal of Nervous and Mental Disease, 170: 415-419.

Willis, W. (1982). Control of nociceptive transmission in the spinal cord, Progress in Sensory Physiology, 3, Berlin: Springer-Verlag.

Wolf, S., Basmajian, J., Russ, T. \& Kutner, M., (1979). Normative data on low back mobility and activity levels, American Journal of physical medicine, 58: 217-229.

Wolff, B., (1980). Measurement of human pain, In: Banica, J.J., Pain, New York: Raven Press: $173-189$.

Wolskee, P. \& Gracely, R., (1980). Effects of chronic pain on experimental pain response, Pain Society Abstracts, $4: 4$.

Woolf, C., (1980). Analgesia and hyperalgesia produced in the rat by intrathecal nalloxone. Brain Research, 189: 593-597.

Wyke, B., (1987). The neurology of low back pain, In: Javson, M.l.V., The lumbar spine arnd back pain, Edinburgh: Churchill Livingstone: 56 -99.

Yang, J., Richlin, D., Brand, L., Wagner, J. \& CLark, C., (1985). Thermal sensory decision theory indices and pain threshold in chronic pain patients and healthy volunteers, Psychosomatic Medicine, 47: $461-468$

Zborowski, M. (1952). Cultural components in responses to pain, Journal of Social /ssues. 8: 16-30.

Zelman, D., Howland. E., Nichols, S. Cleeland, C., (1991). The effects of induced mood on laboratory pain, Pain, 46: 105-111. 



\section{LIST OF ABBREVIATIONS}

\begin{tabular}{|c|c|}
\hline$A L$ & adaptation level \\
\hline $\mathrm{BP}$ & blood pressure \\
\hline CLBP & chronic low back pain \\
\hline CON neurons & convergent meurons \\
\hline $\mathrm{CS}$ & conditioned stimulus \\
\hline $\mathrm{CSF}$ & cerebrospinal fluid \\
\hline DLF & dorsolateral funiculus \\
\hline DNIC & diffuse noxious inhibitory controls \\
\hline DR & defensive retlex \\
\hline DT & detection threshold \\
\hline ECG & electrocardiogram \\
\hline EEG & electro-encephalogram \\
\hline EMG & electromyogram \\
\hline $\mathrm{EOG}$ & electro-oculogram \\
\hline EP & evoked potentials \\
\hline FPV & finger pulse volume \\
\hline FPVR & finger pulse volume response \\
\hline HR & heart rate \\
\hline HRR & heart rate response \\
\hline IASP & International Association for the Study of Pain \\
\hline IBI & inter beat interval \\
\hline IBQ & illness behaviour questionnaire \\
\hline MANOVA & multivariate analysis of variance \\
\hline MMPL & Minnesota Multiphasic Personality Questionnaire \\
\hline $\mathrm{MPQ}$ & McGill Pain Questionnaire \\
\hline MPT & maximal pain tolerance \\
\hline NRM & nucleus raphe magnus \\
\hline NS neurons & nociceptive specific neurons \\
\hline NSAID & non-steroid anti-inflammatory drugs \\
\hline NVM & Nederlandse verkorte MMPI \\
\hline OR & orienting reflex \\
\hline PPT & pain perception threshold \\
\hline RIII-reflex & nociceptiwe reflex involving type III afferents \\
\hline RR & respiration rate \\
\hline $\mathbb{R T}$ & response tendencies \\
\hline SCL & skin conductance level \\
\hline SCR & skin conductance response \\
\hline SDT & signal detection theory \\
\hline S-R pathway & stimulus-response pathway \\
\hline SNS & sympathetic nervous system \\
\hline STAI & Spielberger trait anxiety inventory \\
\hline TENS & transculaneous electro nerve stimulation. \\
\hline UCR & unconditioned response \\
\hline UCS & conditioned response \\
\hline VARCOEF & variation coefficient \\
\hline VAS & visual analog scale \\
\hline
\end{tabular}





\section{DANKWOORD}

Verschillende mensen hebben hun bijgedrage geleverd aan het totstandkomen van dit proefschrift. In de eerste plaats denk ik daarbij aan al die proefpersonen die bereid waren om aan pijnonderzoek deel te nemen. Daarnaast wil ik een aantal mensen met name noemen voor hun steun gedurende de ruim 4 jaar dat het promotie-onderzoek geduurd heeft. Allereerst natuurlijk mijn begeleider en co-promotor Ton Sclmidt. $1 k$ ben hem erkentelijk voor de vrijheid die ik heb gekregen bij het opzetten en uitvoeren van de verschillende experimenten. Hij stond immer open voor mijn plannen en ik kon steeds op zijn steun rekenen. Mijn artikelen en overige stukken werden door hem van deskundig commentaar voorzien. Ik heb onze samenwerking zeer gewaardeerd.

Mijn promotor Marcel van den Hout bedank ik voor de manier waarop hij mij heeft weten te motiveren. Door met hem te praten keerde telkens weer het plezier in de wetenschap terug. Daarnaast slaagde hij er keer op keer in om binnen zeer korte tijd mijn artikelen en hoofdstukken door te lezen en te becommentariëren. Zijn opmerkingen zijn zeer waardevol gebleken.

Verder bedank ik Prof. dr. Jolles, Prof. dr. Sluijter, Prof. dr. Reneman, Prof dr. Everaerd en Prof. dr. Le Bars voor hun bereidheid om zitting te nemen in de beoordelingscommissie. Dinanda Kolbach, Taco Eisenga en Petra Draaijer bedank ik voor hun aandeel in de laatste studie. $\mathrm{Z}_{\mathrm{ij}}$ zorgden woor de noodzakelijke medische ondersteuning. Alan Ralston heeft nagenoeg het gehele proefschrift doorgelezen op fouten in het engels. Gerry van Wunnik en Theo van Aerts stonden altijd klaar om de problemen die zich in het gedragslab voordeden op te lossen. Ook jullie wil ik bedanken.

Ruud Koopmans en de overige medewerkers van de afdeling kaakchirurgie van het AZM bedank ik voor hun medewerking bij het onderzoek bij kaakchirurgie patienten.

De staf en de overige leden van het netwerk "Experimentele Psychopathologie" ben ik erkentelijk voor de stimulerende sfeer binnen de groep. Ondanks mijn aanvankelijke scepsis bleken de netwerk-bijeenkomsten een nuttige en zeker ook plezierige aanvulling op de wetenschappelijke vorming.

Tenslotte bedank ik al mijn collega's van Medische Psycholgie en GGK voor de plezierige tijd die ik bij hen heb doorgebracht en voor hun bereidheid om op te draven als proefkonijn in mijn pilot-studies. 



\section{CURRICULUM VITAE}

Madelon Peters werd geboren op 25 juni 1962 te Amsterdam. In 1980 behaalde zij het VWO diploma aan het Pieter Nieuwland College in Amsterdam. In datzelfde jaar begon zij aan de studie Psychologie aan de Universiteit van Amsterdam. Na het kandidaats-examen werd de afstudeerrichting psychofysiologie gevolgd. In januari 1987 slaagde zij cum laude voor het doctoraal-examen.

Van september 1986 tot april 1987 vervulde zij een onderwijsfunctie bij de afdeling neurofysiologie van de Uniwersiteit van Amsterdam. In april 1987 trad zij als assistent in opleiding in dienst bij de vakgroep Medische Psychologie van de Rijksuniversiteit Limburg, alwaar het in dit proefschrift beschreven onderzoek werd verricht.

Sinds november 1991 is de auteur in dienst van het Instituut voor Geneesmiddelen, Veiligheid en Gedrag te Maastricht. 